\title{
12. LATE EARLY CRETACEOUS RADIOLARIA FROM DEEP SEA DRILLING PROJECT LEG 62 ${ }^{1}$
}

\author{
André Schaaf, Institut de Géologie, 67084 Strasbourg, France \\ Dedicated to Helen Foreman
}

\begin{abstract}
Well-preserved Mesozoic radiolarian faunas have been recovered at four sites of Deep Sea Drilling Project Leg 62 . Late Early Cretaceous assemblages, which occur always with foraminifers or calcareous nannoplankton, allow the description of 21 new species, the introduction of a new zone scheme, and calibration of the radiolarian zones with the geochronological scale.
\end{abstract}

\section{INTRODUCTION}

The locations of the drill sites occupied during DSDP Leg 62 are as follows (Fig. 1):

Site $463: 21^{\circ} 21.01^{\prime} \mathrm{N}, 174^{\circ} 40.07^{\prime} \mathrm{E}$, water depth 2525 meters,

Site $464: 39^{\circ} 51.64^{\prime} \mathrm{N}, 173^{\circ} 53.33^{\prime} \mathrm{E}$, water depth 4637 meters,

Site $465: 33^{\circ} 49.23^{\prime} \mathrm{N}, 178^{\circ} 55.14^{\prime} \mathrm{E}$, water depth 2161 meters,

Site $466: 34^{\circ} 11.46^{\prime} \mathrm{N}, 179^{\circ} 15.34^{\prime} \mathrm{E}$, water depth 2665 meters.

Sites 464,465 , and 466 were drilled on Hess Rise; Site 463 was drilled on the Mid-Pacific Mountains.

The long sequence of well-preserved late Early Cretaceous radiolarians (always with calcareous fossils) at Site 463 is of considerable significance and is the basis for most of this report. Figure 2 shows the biostratigraphic synthesis based on foraminifers and calcareous nannoplankton. Cenozoic radiolarians, often poorly preserved, were not studied for this paper.

\section{DISTRIBUTION OF RADIOLARIANS AT EACH SITE}

\section{Site 463 (Table 1)}

Three intervals can be defined in the sedimentary sequence at Site 463 on the basis of radiolarian occurrences and preservation:

1) In Cores 1 to 56 , radiolarians are nearly absent, except in Cores 52, 53, and 56 (middle to late Albian), which yielded a poorly preserved fauna.

2) In Cores 57 through 74 , radiolarians are confined to certain layers. The tests are usually recrystallized, and many internal molds of iron oxide or pyrite (with or without the original skeleton) were observed. In general, down to Core 63 the internal molds are iron oxide, and below they are pyrite.

\footnotetext{
${ }^{1}$ Initial Reports of the Deep Sea Drilling Project, Volume 62.
}

3) In Cores 75 to 92 , mostly well-preserved radiolarians occur consistently throughout the section. Internal molds of pyrite were also observed in this interval.

\section{Site 464 (Table 2)}

At Site 464 Cenozoic radiolarians were abundant and generally well preserved in the first five Cores, whereas Cretaceous radiolarians, although occasionally common, are badly preserved. Only core-catcher samples from Cores 13, 15 and 16 contain identifiable radiolarians.

\section{Site 465 (Table 3)}

At this Site only the first two cores of Hole 465 (up to $2-2-120$ ) contained common to rare, and poorly preserved Cenozoic radiolarians. The Cenozoic section of Hole $465 \mathrm{~A}$ is entirely barren. Cretaceous radiolarians occur only at two levels: a sparse and poorly preserved upper Santonian fauna in 465A-21,CC (not studied in this paper); and an abundant and well-preserved upper Albian fauna in 465A-29-1, 43-44 cm.

\section{Site 466 (Table 4)}

At Site 466, two distinct radiolarian assemblages were recovered: a Pliocene to Quaternary sequence in the upper 61 meters, and a Cretaceous sequence in the lower 85 meters.

Cores 29 to 34 contain an upper Albian fauna, usually sparse and moderately well preserved, but abundant and well preserved in Core 34 .

Table 5 shows those samples in which no Early Cretaceous radiolarians were found.

\section{LATE EARLY CRETACEOUS ZONATION}

Several researchers recently contributed to a better knowledge of Early Cretaceous radiolarian stratigraphy (Foreman, 1973b, 1975; Moore, 1973; Riedel and Sanfilippo, 1974; Pessagno, 1977b).

We have followed in this paper the works of Riedel and Sanfilippo (1974) and Foreman (1975). Correlation 


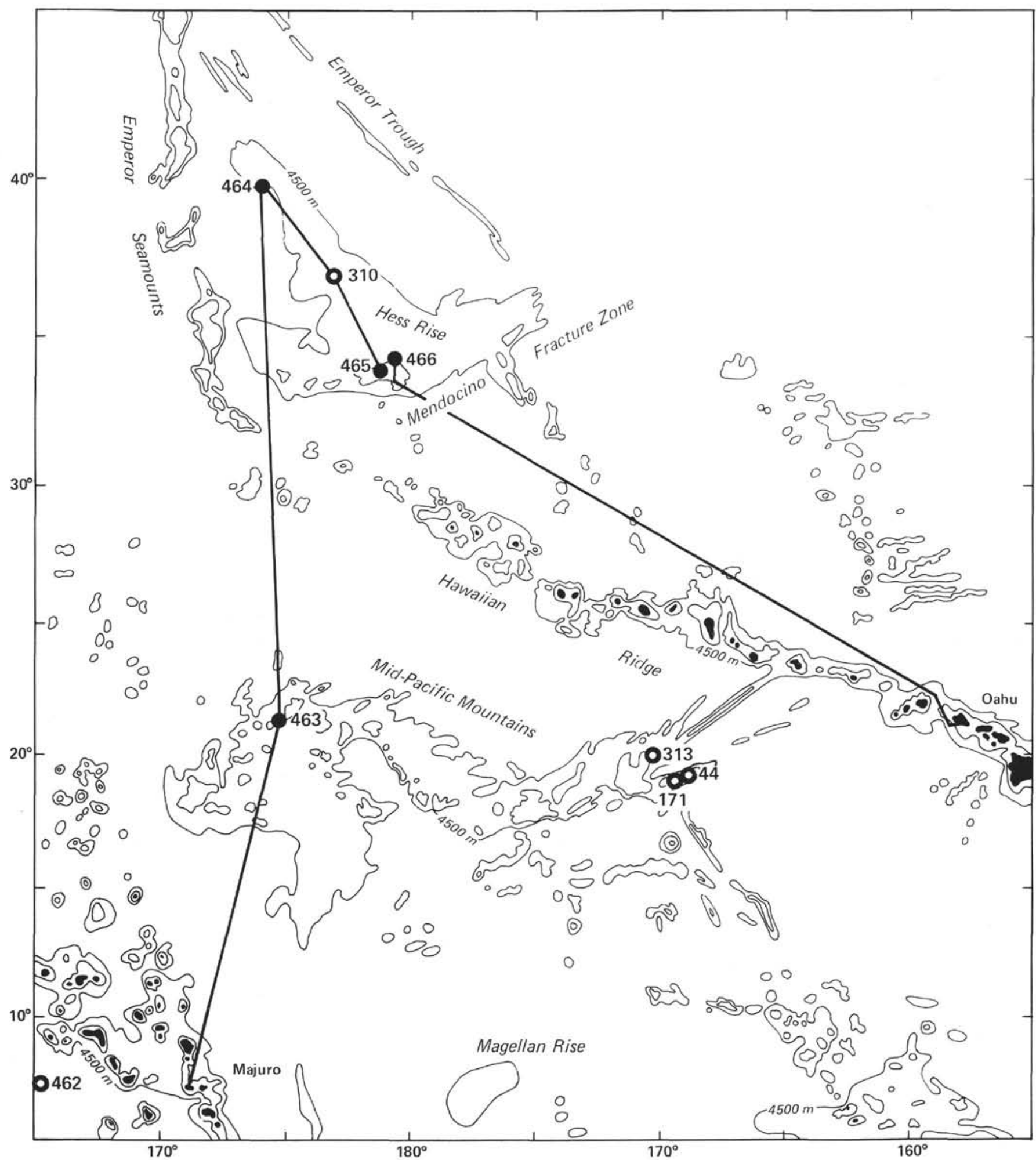

Figure 1. Location of sites drilled on DSDP Leg 62 (solid circles), and previous sites (open circles).

with Pessagno's (1977b) zonation of the Lower Cretaceous of California was more difficult.

Three new zones are proposed to replace the Eucyrtis tenuis Zone of Foreman (1975) (part of the Stichocapsa tenuis Zone of Riedel and Sanfilippo, 1974): the Dibo- lachras tytthopora Zone, the Crolanium pythiae Zone, and the Archicapsa similis Zone.

The top of each zone is defined by the base of the overlying zone. Figures 3 and 4 show the relationships between species ranges and zone boundaries. 


\begin{tabular}{|c|c|c|c|c|c|c|c|c|}
\hline \multirow[b]{2}{*}{ Stage } & \multicolumn{2}{|c|}{ Site 463} & \multicolumn{2}{|c|}{ Site 464} & \multicolumn{2}{|c|}{$\begin{array}{c}\text { Site } 465 \\
\text { (Hole 465A) }\end{array}$} & \multicolumn{2}{|c|}{ Site 466} \\
\hline & $\begin{array}{l}\text { Core and } \\
\text { Section }\end{array}$ & $\begin{array}{l}\text { Sub-bottom } \\
\text { Depth (m) }\end{array}$ & $\begin{array}{c}\text { Core and } \\
\text { Section }\end{array}$ & $\begin{array}{l}\text { Sub-bottom } \\
\text { Depth (m) }\end{array}$ & $\begin{array}{l}\text { Core and } \\
\text { Section }\end{array}$ & $\begin{array}{l}\text { Sub-bottom } \\
\text { Depth (m) }\end{array}$ & $\begin{array}{l}\text { Core and } \\
\text { Section }\end{array}$ & $\begin{array}{c}\text { Sub-bottom } \\
\text { Depth (m) }\end{array}$ \\
\hline Lower Cenomanian & $\begin{array}{l}43-1 \\
45-1 \\
\end{array}$ & $\begin{array}{l}378 \\
404.5 \\
\end{array}$ & $\begin{array}{l}11-1 \\
17-1 \\
\end{array}$ & $\begin{array}{r}89 \\
146 \\
\end{array}$ & $\begin{array}{l}26-1 \\
27, \mathrm{CC}\end{array}$ & $\begin{array}{l}276.5 \\
295.5 \\
\end{array}$ & \multicolumn{2}{|c|}{ (Hiatus) } \\
\hline Upper Albian & $\begin{array}{l}46-1 \\
52, \mathrm{CC}\end{array}$ & $\begin{array}{l}404.5 \\
461.5 \\
\end{array}$ & $\begin{array}{l}17-1 \\
24-1 \\
\end{array}$ & $\begin{array}{l}146 \\
222 \\
\end{array}$ & $\begin{array}{l}28-1 \\
40-1 \\
\end{array}$ & $\begin{array}{l}295.5 \\
411.7 \\
\end{array}$ & $\begin{array}{l}29-1 \\
35, \mathrm{CC}\end{array}$ & $\begin{array}{l}255 \\
312 \\
\end{array}$ \\
\hline Middle Albian & $\begin{array}{l}53-1 \\
55-1 \\
\end{array}$ & $\begin{array}{l}461.5 \\
481.5 \\
\end{array}$ & $\begin{array}{l}25, \mathrm{CC} \\
26, \mathrm{CC} \\
\end{array}$ & $\begin{array}{l}222 \\
241 \\
\end{array}$ & & & \multicolumn{2}{|c|}{ (Trachyte) } \\
\hline Lower Albian & $\begin{array}{l}55-1 \\
59, \mathrm{CC}\end{array}$ & $\begin{array}{l}481.5 \\
528\end{array}$ & $\begin{array}{l}27-1 \\
34, \mathrm{CC}\end{array}$ & $\begin{array}{l}241 \\
308.5 \\
\end{array}$ & & & & \\
\hline Upper Aptian & $\begin{array}{l}60-1 \\
65-2 \\
\end{array}$ & $\begin{array}{l}528 \\
558 \\
\end{array}$ & \multicolumn{2}{|c|}{ (Basalt) } & & & & \\
\hline Lower Aptian & $\begin{array}{l}65, \mathrm{CC} \\
78-1 \\
\end{array}$ & $\begin{array}{l}558 \\
699 \\
\end{array}$ & & & & & & \\
\hline Upper Barremian & $\begin{array}{l}79-1 \\
92-1\end{array}$ & $\begin{array}{l}699 \\
822.5\end{array}$ & & & & & & \\
\hline
\end{tabular}

Figure 2. Age assignments of Leg 62 cores, based on synthesis of data on calcareous-nannoplankton and foraminifer assemblages (after Čepek and Boersma, this volume).

\section{Obesacapsula somphedia Zone (Foreman, 1975)}

The base is defined by the earliest morphotypic appearance of Obesacapsula somphedia (Foreman, 1975).

Events within the zone include the earliest appearances of Stichocapsa zamoraensis, Orbiculiforma chartonae, Histiastrum aster, Parvicingula? tekschaensis, Rhopalosyringium majuroensis, and Theocapsomma ancus; and the latest occurrences of Theocorys antiqua; and the entire ranges of Pseudodictyomitra pseudomacrocephala, Holocryptocanium barbui, Mita magnifica, and Excentropylomma cenomana.

\section{Acaeniotyle umbilicata Zone (Foreman, 1975)}

The base is defined by the extinction of Sphaerostylus lanceola, which may be approximately synchronous with the extinction of Archaeodictyomitra lacrimula, Dicroa sp. A, Sethocapsa orca, and Triactoma hybum. It may be desirable to redefine the base of this zone in terms of presence of species. However, in this study no suitable species could be found.

Events within the zone include the earliest appearance of Theocorys antiqua and the latest occurrence of Archicapsa similis.

\section{Archicapsa similis Zone (Schaaf, new zone)}

The base is defined by the earliest morphotypic appearance of Archicapsa similis, approximately synchronous with the latest occurrences of Holocryptocapsa hindei, Pseudodictyomitra leptoconica, and Williriedellum peterschmittae.

Events within the zone include the latest occurrences of Crolanium pythiae, Dibolachras tytthopora, and Acanthocircus carinatus.

\section{Crolanium pythiae Zone (Schaaf, new zone)}

The base is defined by the earliest morphotypic appearance of Crolanium pythiae, synchronous with the latest occurrence of Thanarla pulchra, Podobursa tricola, and Archaeodictyomitra apiara.

Events within the zone include the latest occurrence of Saitoum cepecki, Alievum helenae, and Sethocapsa uterculus.

\section{Dibolachras tytthopora Zone (Schaff, new zone)}

The base is defined by the earliest morphotypic appearance of Dibolachras tytthopora, approximately synchronous with the earliest appearance of Acanthocircus carinatus, Archaeodictyomitra lacrimula, and Eucyrtis tenuis.

Events within the zone include the latest occurrences of Lithocampe chenodes, Cyrtocalpis operosa, Acanthocircus dicranacanthos, and Staurosphaera septemporata.

\section{Staurosphaera septemporata Zone (Riedel and Sanfilippo, 1974)}

The base is defined by the earliest morphotypic appearance of Staurosphaera septemporata, and the top by the latest occurrence of Stichocapsa cribata.

The Dibolachras tytthopora Zone, Crolanium pythiae Zone, and Archicapsa similis Zone result from splitting of the Eucyrtis tenuis Zone of Riedel and Sanfilippo (1974; emend. Foreman, 1975). The Barremian/ Aptian boundary coincides with the base of the $\mathrm{Ar}$ chicapsa similis Zone (Fig. 4). This correspondence is corroborated by the Geomagnetic Polarity Zone M1 and the base of the Chiastozygus litterarius Zone which begins in the uppermost Barremian (H. Thierstein, pers. comm.).

\section{SYSTEMATICS}

The following procedures were used in this work:

1) Spumellaria were subdivided according to the suprageneric classification of Riedel (1971), with the exception that the Porodiscidae were split from the Spongodiscidae (Petrushevskaya and Kozlova, 1972), and the Hagiastridae were elevated to the family level (Pessagno, 1971). 
Table 1. Occurrence and abundance of late Early Cretaceous radiolarians of Site 463.

\begin{tabular}{|c|c|c|c|c|c|c|c|c|c|c|c|c|c|c|c|c|c|c|c|c|c|c|c|c|c|c|}
\hline $\begin{array}{c}\text { Sample } \\
\text { (interval in } \mathrm{cm} \text { ) }\end{array}$ & 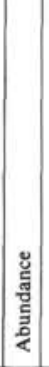 & 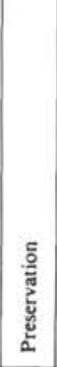 & 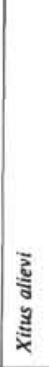 & 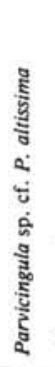 & 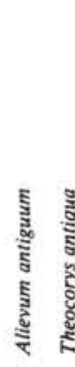 & 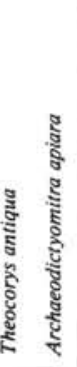 & 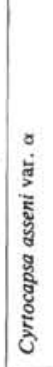 & $\begin{array}{l}3 \\
\text { हूँ } \\
\text { हू } \\
\text { हूँ } \\
\text { हू } \\
\text { हूँ }\end{array}$ & 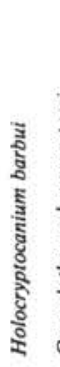 & 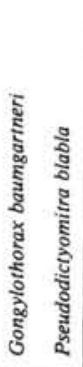 & 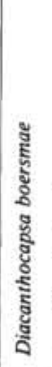 & 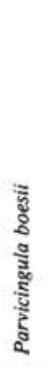 & 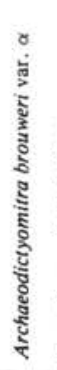 & 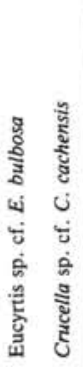 & 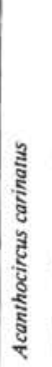 & 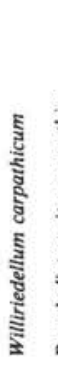 & 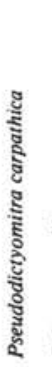 & 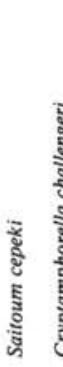 & 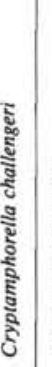 & 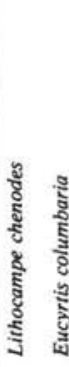 & 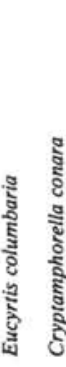 & 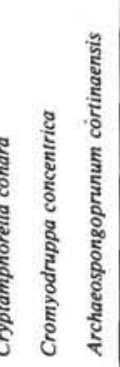 & 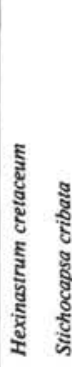 & 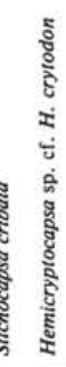 & 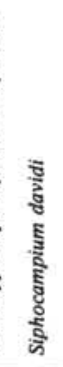 & 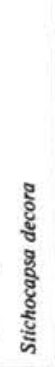 \\
\hline $\begin{array}{l}463-52, C C \\
53-1,130-131 \\
56-1,27-28 \\
58-2,125-126 \\
59-2,10-11 \\
\end{array}$ & \begin{tabular}{|l|}
$\mathrm{F}$ \\
$\mathrm{F}$ \\
$\mathrm{C}$ \\
$\mathrm{C}$ \\
$\mathrm{C}$ \\
\end{tabular} & \begin{tabular}{|c|}
$P$ \\
$P M$ \\
$P M$ \\
$P$ \\
$P M$ \\
\end{tabular} & $\bar{z}$ & & & $\begin{array}{l} \\
\mathrm{R} \\
\mathrm{R} \\
\mathrm{R} \\
\end{array}$ & & $\begin{array}{l}\bar{z} \\
\bar{z}\end{array}$ & $\begin{array}{l}\bar{r} \\
\stackrel{R}{\mathrm{R}} \\
\overline{-} \\
-\end{array}$ & & & & & & & & $\begin{array}{l}\bar{R} \\
R \\
\bar{R} \\
\end{array}$ & & & & & $\begin{array}{ll}\mathbf{F} & \mathbf{R} \\
\mathbf{R} & \mathbf{R} \\
& = \\
= & = \\
\end{array}$ & & & & \\
\hline $\begin{array}{l}60-2,42-43 \\
61-1,29-30 \\
62-1,53-54 \\
62-1,91-92 \\
62-1,148-149 \\
\end{array}$ & \begin{tabular}{|l|}
$\mathrm{A}$ \\
$\mathrm{A}$ \\
$\mathrm{A}$ \\
$\mathrm{A}$ \\
$\mathrm{A}$ \\
\end{tabular} & $\begin{array}{l}\mathrm{M} \\
\mathrm{M} \\
\mathrm{M} \\
\mathrm{M} \\
\mathrm{M} \\
\end{array}$ & $\begin{array}{l}\bar{R} \\
\mathrm{R} \\
\mathrm{F} \\
\\
\end{array}$ & & & R & & $\begin{array}{l}\bar{z} \\
\bar{z}\end{array}$ & - & & & & & & & & $\begin{array}{l}\mathbf{R} \\
+ \\
\mathbf{R} \\
\mathrm{R} \\
- \\
\end{array}$ & & & & & & & & & \\
\hline $\begin{array}{l}62-2,15-16 \\
62-2,119-120 \\
63, C C \\
64-1,66-67 \\
64-2,31-32 \\
\end{array}$ & \begin{tabular}{|l|}
$\mathrm{A}$ \\
$\mathrm{A}$ \\
$\mathrm{A}$ \\
$\mathrm{A}$ \\
$\mathrm{A}$ \\
\end{tabular} & \begin{tabular}{|c|}
$\mathrm{M}$ \\
$\mathrm{M}$ \\
$\mathrm{M}$ \\
$\mathrm{PM}$ \\
$\mathrm{PM}$ \\
\end{tabular} & $\begin{array}{l}\mathrm{R} \\
\mathrm{R} \\
\mathrm{F} \\
- \\
\\
\end{array}$ & & & & & $\begin{array}{l}\mathrm{R} \\
\pm \\
\pm \\
\end{array}$ & & & & & & & & & $\begin{array}{l}+ \\
\mathbf{R} \\
\mathbf{R} \\
\mathrm{R} \\
- \\
\end{array}$ & & & & & & & & & \\
\hline $\begin{array}{l}64-3,31-32 \\
65-2,9-10 \\
66-1,27-28 \\
66-2,27-28 \\
66-3,21-22 \\
\end{array}$ & \begin{tabular}{l|} 
A \\
A \\
A \\
A \\
C \\
\end{tabular} & $\begin{array}{l}\mathrm{PM} \\
\mathrm{PM} \\
\mathrm{PM} \\
\mathrm{PM} \\
\mathrm{M} \\
\end{array}$ & $\begin{array}{l}\mathrm{R} \\
\mathrm{F} \\
\mathrm{F} \\
\mathrm{F} \\
\mathrm{R} \\
\end{array}$ & & & & & $\begin{array}{l}\bar{z} \\
\pm \\
\pm\end{array}$ & & & & & & & & & $\begin{array}{l}\mathrm{R} \\
\mathrm{R} \\
\mathrm{F} \\
\mathrm{F} \\
+ \\
\end{array}$ & & & & & & & & & \\
\hline $\begin{array}{l}67-1,6-7 \\
67-2,110-111 \\
69-2,90-91 \\
69, C C \\
70-1,35-36 \\
\end{array}$ & \begin{tabular}{l|l}
$\mathrm{C}$ \\
$\mathrm{A}$ \\
$\mathrm{R}$ \\
$\mathrm{C}$ \\
$\mathrm{C}$ \\
\end{tabular} & \begin{tabular}{l|}
$\mathrm{P}$ \\
$\mathrm{P}$ \\
$\mathrm{P}$ \\
$\mathrm{P}$ \\
$\mathrm{P}$ \\
\end{tabular} & $\begin{array}{l}\mathrm{R} \\
\mathrm{F} \\
\mathrm{R} \\
\mathrm{R} \\
\mathrm{F} \\
\end{array}$ & & & & & $\begin{array}{l}\bar{z} \\
\bar{z} \\
\bar{z}\end{array}$ & & & & $\bar{z}$ & & $\overline{-}$ & & & $\begin{array}{l}+ \\
R \\
R \\
R \\
R \\
R \\
\end{array}$ & & & & - & & & & & \\
\hline $\begin{array}{l}70-5,28-29 \\
71-2,99-100 \\
71-3,54-55 \\
72-1,43-44 \\
72-3,14-15 \\
\end{array}$ & \begin{tabular}{|l|}
$\mathrm{F}$ \\
$\mathrm{A}$ \\
$\mathrm{A}$ \\
$\mathrm{A}$ \\
$\mathrm{A}$ \\
\end{tabular} & \begin{tabular}{c|}
$\mathrm{P}$ \\
$\mathrm{PM}$ \\
$\mathrm{M}$ \\
$\mathrm{M}$ \\
$\mathrm{M}$ \\
\end{tabular} & $\begin{array}{ll}\mathrm{F} \\
\mathrm{C} \\
\mathrm{C} \\
\mathrm{C} \\
\mathrm{C} \\
\end{array}$ & & & & & $\begin{array}{l}\mathrm{R} \\
\mathrm{R} \\
- \\
= \\
\end{array}$ & & & & $\begin{array}{l}\bar{R} \\
\mathrm{R} \\
\mathrm{F} \\
\mathrm{R} \\
\end{array}$ & & $\begin{array}{l}\bar{R} \\
R \\
F \\
R\end{array}$ & & & $\begin{array}{l}- \\
+ \\
R \\
+ \\
+ \\
R\end{array}$ & & & & $\begin{array}{l}\bar{z} \\
\bar{z} \\
\bar{z}\end{array}$ & & & & & \\
\hline $\begin{array}{l}72-5,20-21 \\
72, \mathrm{CC} \\
73-1,18-19 \\
73-1,140-141 \\
73-2,18-19 \\
\end{array}$ & \begin{tabular}{|l|}
$\mathrm{F}$ \\
$\mathrm{C}$ \\
$\mathrm{A}$ \\
$\mathrm{C}$ \\
$\mathrm{F}$ \\
\end{tabular} & $\begin{array}{l}\mathrm{PM} \\
\mathrm{M} \\
\mathrm{M} \\
\mathrm{P} \\
\mathrm{P} \\
\end{array}$ & $\begin{array}{l}\mathrm{R} \\
\mathrm{F} \\
\mathrm{F} \\
\mathrm{F} \\
\mathrm{R} \\
\end{array}$ & & & & & $\begin{array}{l}\bar{z} \\
\bar{R} \\
\end{array}$ & & & & $\begin{array}{l}\bar{R} \\
\mathrm{R} \\
\mathrm{R} \\
-\end{array}$ & & $\begin{array}{l}\mathrm{R} \\
\mathrm{R} \\
\mathrm{R} \\
- \\
- \\
\end{array}$ & $\begin{array}{l}\bar{z} \\
\bar{z}\end{array}$ & & $\begin{array}{l}\bar{R} \\
R \\
R \\
- \\
\end{array}$ & & & & $\begin{array}{l}\mathrm{R} \\
\mathrm{R} \\
\mathrm{R} \\
\mathrm{R} \\
- \\
\end{array}$ & & $\overline{-}$ & & & \\
\hline $\begin{array}{l}73-3,19-20 \\
73-4,10-11 \\
73-4,116-117 \\
74-1,110-111 \\
75-1,21-22\end{array}$ & \begin{tabular}{|c|}
$\mathrm{F}$ \\
$\mathrm{F}$ \\
$\mathrm{F}$ \\
$\mathrm{F}$ \\
$\mathrm{A}$ \\
\end{tabular} & \begin{tabular}{c|}
$P$ \\
$P M$ \\
$M$ \\
$M$ \\
$M$ \\
\end{tabular} & $\begin{array}{l}\bar{R} \\
\bar{R} \\
\mathrm{R}\end{array}$ & & & & & $\begin{array}{l}\overline{-} \\
\bar{R} \\
\mathrm{~F}\end{array}$ & & & & $\frac{+}{\mathrm{R}}$ & $\begin{array}{l}- \\
\bar{R}\end{array}$ & $\bar{z}$ & $\begin{array}{l}R \\
+ \\
+ \\
R \\
+ \\
+\end{array}$ & & $\begin{array}{l}\bar{z} \\
\bar{z} \\
\bar{R}\end{array}$ & & & & $\begin{array}{l}\bar{I} \\
\overline{+} \\
\bar{R}\end{array}$ & & $\begin{array}{l}\overline{-} \\
\bar{R} \\
R \\
F \\
\end{array}$ & & & \\
\hline $\begin{array}{l}76, C C \\
77-1,7-8 \\
77-1,60-61 \\
78-1,72-73 \\
78-1,111-112 \\
\end{array}$ & $\begin{array}{l}\text { C } \\
\text { A } \\
\text { C } \\
\text { A } \\
\text { A }\end{array}$ & \begin{tabular}{c|}
$\mathrm{P}$ \\
$P$ \\
$\mathrm{PM}$ \\
$\mathrm{PM}$ \\
$\mathrm{PM}$
\end{tabular} & $\begin{array}{l}\mathrm{F} \\
\mathrm{C} \\
\mathrm{F} \\
\mathrm{C} \\
\mathrm{C}\end{array}$ & & & & & $\begin{array}{l}\bar{z} \\
\bar{z}\end{array}$ & & & & $\begin{array}{l}\mathrm{F} \\
\mathrm{F} \\
\mathrm{R} \\
+ \\
\mathrm{R} \\
\end{array}$ & $\begin{array}{l}\mathrm{R} \\
\mathrm{R} \\
\mathrm{F} \\
\mathrm{C} \\
\mathrm{C} \\
\end{array}$ & & $\begin{array}{l}\mathrm{R} \\
\mathrm{R} \\
\mathrm{R} \\
\mathrm{F} \\
\mathrm{F} \\
\end{array}$ & & $\begin{array}{l}+ \\
R \\
R \\
F \\
F \\
\end{array}$ & & & & $\begin{array}{l}\mathrm{R} \\
\mathrm{F} \\
\mathrm{F} \\
+ \\
\mathrm{R} \\
\end{array}$ & & $\begin{array}{l} \pm \\
\pm \\
-\end{array}$ & & & \\
\hline $\begin{array}{l}79-1,36-37 \\
80-1,59-60 \\
81-2,45-46 \\
82, C C \\
83-1,0-1\end{array}$ & $\begin{array}{l}\mathrm{R} \\
\mathrm{R} \\
\mathrm{R} \\
\mathrm{A} \\
\mathrm{A}\end{array}$ & \begin{tabular}{l|}
$\mathrm{P}$ \\
$\mathrm{P}$ \\
$\mathrm{P}$ \\
$\mathrm{M}$ \\
$\mathrm{M}$
\end{tabular} & $\begin{array}{l}- \\
\bar{R} \\
\mathrm{~F} \\
\mathrm{C}\end{array}$ & & & - & & $\frac{\bar{R}}{\mathrm{R}}$ & & & & $\begin{array}{l}\bar{R} \\
\bar{R} \\
F\end{array}$ & $\begin{array}{l}\mathrm{C} \\
\mathrm{F} \\
\mathrm{F} \\
\mathrm{C} \\
\mathrm{F}\end{array}$ & & $\begin{array}{l}\bar{z} \\
\bar{Z} \\
R \\
R\end{array}$ & & $\begin{array}{l}\bar{z} \\
\bar{z} \\
\mathrm{R} \\
\mathrm{R}\end{array}$ & $\begin{array}{l}z \\
z\end{array}$ & & & $\begin{array}{l}\mathrm{F} \\
\mathrm{F} \\
\mathrm{F} \\
\mathrm{F}\end{array}$ & & & & & \\
\hline $\begin{array}{l}83-1,38-39 \\
83-1,130-131 \\
84-1,5-6 \\
84-1,19-20 \\
84, C C \\
\end{array}$ & \begin{tabular}{c|}
$\mathrm{A}$ \\
$\mathrm{R}$ \\
$\mathrm{A}$ \\
$\mathrm{F}$ \\
$\mathrm{F}$
\end{tabular} & \begin{tabular}{c|}
$\mathrm{M}$ \\
$\mathrm{M}$ \\
$\mathrm{M}$ \\
$\mathrm{M}$ \\
$\mathrm{PM}$ \\
\end{tabular} & $\begin{array}{l}\mathrm{C} \\
\mathrm{C} \\
\mathrm{F} \\
\mathrm{F}\end{array}$ & & & $\begin{array}{l}\overline{-} \\
\bar{?} \\
-\end{array}$ & & $\begin{array}{l}= \\
= \\
=\end{array}$ & & & $\overline{-}$ & $\begin{array}{l}\mathrm{F} \\
\mathrm{R} \\
\mathrm{R} \\
\mathrm{F} \\
+ \\
\end{array}$ & $\begin{array}{l}\mathrm{C} \\
\mathrm{C} \\
\mathrm{C} \\
\mathrm{F} \\
\mathrm{F} \\
\end{array}$ & & $\begin{array}{l}+ \\
+ \\
+ \\
F \\
F \\
R \\
\end{array}$ & & $\begin{array}{l}\frac{R}{R} \\
- \\
\end{array}$ & $\begin{array}{l}\bar{R} \\
+ \\
\overline{-} \\
\end{array}$ & $\begin{array}{l}- \\
-\end{array}$ & & $\begin{array}{l}\mathrm{C} \\
\mathrm{C} \\
\mathrm{F} \\
\mathrm{C} \\
\mathrm{C} \\
\end{array}$ & & & & & \\
\hline \begin{tabular}{l|}
$85-1,19-20$ \\
$85-1,142-143$ \\
$85-2,20-21$ \\
$86-1,31-32$ \\
$86-1,115-116$ \\
\end{tabular} & \begin{tabular}{l|l}
$F$ \\
$F$ \\
$F$ \\
$F$ \\
$F$
\end{tabular} & \begin{tabular}{c|}
$\mathrm{P}$ \\
$\mathrm{PM}$ \\
$\mathrm{M}$ \\
$\mathrm{P}$ \\
$\mathrm{M}$ \\
\end{tabular} & $\begin{array}{l}\mathrm{R} \\
\mathrm{R} \\
\mathrm{R} \\
\mathrm{R} \\
- \\
\end{array}$ & & & $\begin{array}{l}R \\
R \\
R \\
R \\
\end{array}$ & & $\begin{array}{l}\overline{+} \\
\bar{z} \\
\bar{R}\end{array}$ & & $\begin{array}{l}\bar{z} \\
\bar{Z} \\
\bar{R} \\
\mathrm{R}\end{array}$ & $\begin{array}{l}\overline{\mathrm{F}} \\
\mathrm{F} \\
\mathrm{R} \\
- \\
\end{array}$ & $\begin{array}{l}\mathrm{R} \\
\mathrm{F} \\
\mathrm{R} \\
-\end{array}$ & $\begin{array}{l}\mathrm{R} \\
\mathrm{R} \\
\mathrm{F} \\
+ \\
\mathrm{F} \\
\end{array}$ & & \begin{tabular}{|l|}
$\mathrm{R}$ \\
$\mathrm{F}$ \\
$\mathrm{F}$ \\
$\mathrm{R}$ \\
\end{tabular} & & $\begin{array}{l}\mathrm{R} \\
\mathrm{R} \\
= \\
= \\
\end{array}$ & $\begin{array}{ll}\mathrm{R} & - \\
& \mathrm{R} \\
+ & \mathrm{R} \\
\mathrm{R} & \mathrm{F} \\
\mathrm{F} & + \\
\end{array}$ & $\begin{array}{l}\bar{R} \\
R \\
R \\
+ \\
\end{array}$ & & $\begin{array}{l}\mathrm{C} \\
\mathrm{C} \\
\mathrm{F} \\
\mathrm{R} \\
\mathrm{R} \\
\mathrm{R}\end{array}$ & & & & & \\
\hline $\begin{array}{l}86, C C \\
87-1,6-7 \\
87-1,65-66 \\
88-1,0-1 \\
88-1,29-30 \\
\end{array}$ & \begin{tabular}{l|l}
$\mathrm{F}$ \\
$\mathrm{F}$ \\
$\mathrm{C}$ \\
$\mathrm{A}$ \\
$\mathrm{F}$
\end{tabular} & \begin{tabular}{c|}
$\mathrm{M}$ \\
$\mathrm{PM}$ \\
$\mathrm{M}$ \\
$\mathrm{PM}$ \\
$\mathrm{P}$
\end{tabular} & $\begin{array}{l}\mathrm{R} \\
\mathrm{C} \\
\mathrm{A} \\
-\end{array}$ & & & $\begin{array}{l}\mathrm{R} \\
\mathrm{R} \\
\mathrm{F} \\
\mathrm{R} \\
\mathrm{F} \\
\end{array}$ & & $\begin{array}{l} \pm \\
\pm \\
=\end{array}$ & & 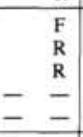 & $\begin{array}{l}\mathrm{R} \\
\bar{Z} \\
\overline{\mathrm{R}}\end{array}$ & $\begin{array}{l}\mathrm{R} \\
\mathrm{F} \\
\mathrm{F} \\
\mathrm{F} \\
- \\
\end{array}$ & $\begin{array}{l}\text { C } \\
\text { C } \\
\text { A } \\
\text { C } \\
\text { C }\end{array}$ & & $\begin{array}{l}\mathrm{R} \\
\mathrm{F} \\
\mathrm{C} \\
\mathrm{F} \\
\mathrm{F} \\
\end{array}$ & & $\begin{array}{l}\mathrm{R} \\
\mathrm{F} \\
\mathrm{F} \\
\mathrm{R}\end{array}$ & $\begin{array}{ll}\mathrm{R} & ? \\
\mathrm{~F} & \mathrm{R} \\
\mathrm{F} & \mathrm{F} \\
\mathrm{F} & + \\
\end{array}$ & \begin{tabular}{l|l}
$?$ & \\
$\mathrm{R}$ & \\
$\mathrm{F}$ & \\
$\mathrm{R}$ & \\
+ & \\
\end{tabular} & 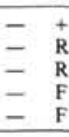 & $\begin{array}{l}++ \\
\mathrm{R} \\
\mathrm{R} \\
\mathrm{F} \\
\mathrm{F} \\
\end{array}$ & & $=$ & & $\begin{array}{l}\bar{z} \\
\bar{F} \\
F\end{array}$ & \\
\hline \begin{tabular}{|l|}
$88-1,52-53$ \\
$89-1,15-16$ \\
$89-1,23-24$ \\
$89-1,94-95$ \\
$89-1,105-106$ \\
\end{tabular} & \begin{tabular}{l|l}
$\mathrm{F}$ \\
$\mathrm{C}$ \\
$\mathrm{A}$ \\
$\mathrm{C}$ \\
$\mathrm{A}$
\end{tabular} & \begin{tabular}{c|c}
$\mathrm{M}$ \\
$\mathrm{M}$ \\
$\mathrm{M}$ \\
$\mathrm{MG}$ \\
$\mathrm{G}$ \\
\end{tabular} & $\begin{array}{l}\mathrm{R} \\
\mathrm{A} \\
\mathrm{A} \\
\mathrm{A} \\
\mathrm{C} \\
\end{array}$ & $\begin{array}{l}= \\
\overline{\mathrm{R}} \\
\end{array}$ & $\begin{array}{l}\overline{-} \\
\overline{\mathrm{R}} \\
\overline{-}\end{array}$ & \begin{tabular}{l|}
$\mathrm{F}$ \\
$\mathrm{R}$ \\
$\mathrm{C}$ \\
$\mathrm{F}$ \\
$\mathrm{F}$ \\
\end{tabular} & $\begin{array}{l}- \\
\overline{-} \\
\bar{R} \\
\mathrm{R} \\
\end{array}$ & $\begin{array}{l}- \\
\bar{z} \\
\bar{R}\end{array}$ & & $\begin{array}{l}\bar{F} \\
R \\
F \\
F\end{array}$ & $\begin{array}{l}\bar{R} \\
R \\
F \\
C \\
\end{array}$ & $\begin{array}{l}+ \\
\mathrm{F} \\
\mathrm{C} \\
\mathrm{C} \\
\mathrm{F}\end{array}$ & $\begin{array}{l}\text { C } \\
\text { A } \\
\text { A } \\
\text { A } \\
\text { A }\end{array}$ & $\begin{array}{l}\overline{-} \\
\overline{-} \\
\bar{R} \\
\mathrm{R}\end{array}$ & $\begin{array}{l}\mathrm{R} \\
\mathrm{R} \\
\mathrm{R} \\
\mathrm{R} \\
- \\
\end{array}$ & $\begin{array}{l}\bar{z} \\
\bar{R}\end{array}$ & $\begin{array}{l}\mathrm{F} \\
\mathrm{F} \\
\mathrm{F} \\
\mathrm{R} \\
\mathrm{R} \\
\end{array}$ & $\begin{array}{ll}\mathrm{F} & \mathrm{R} \\
\mathrm{R} & \mathrm{R} \\
\mathrm{C} & - \\
\mathrm{C} & - \\
\mathrm{C} & - \\
\end{array}$ & \begin{tabular}{l|l}
$\mathbf{R}$ \\
$\mathbf{R}$ \\
\\
\\
- \\
\end{tabular} & $\begin{array}{ll}\bar{T} & \mathrm{C} \\
\mathrm{R} & \mathrm{C} \\
\mathrm{R} & \mathrm{C} \\
\mathrm{R} & \mathrm{C} \\
\mathrm{R} & \mathrm{A} \\
\end{array}$ & $\begin{array}{ll}\text { C } \\
\text { C } \\
\text { c } \\
\text { C } \\
\text { A ? }\end{array}$ & 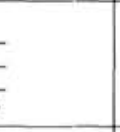 & $\begin{array}{l}\overline{-} \\
\bar{F}\end{array}$ & $=-$ & $\begin{array}{l}- \\
+ \\
F \\
F \\
F\end{array}$ & $\begin{array}{l}\bar{z} \\
\bar{F} \\
\mathrm{R}\end{array}$ \\
\hline $\begin{array}{l}90, \mathrm{CC} \\
91, \mathrm{CC} \\
92-1,0-1 \\
92-1,10-11\end{array}$ & $\begin{array}{l}\mathrm{A} \\
\mathrm{C} \\
\mathrm{F} \\
\mathrm{F}\end{array}$ & $\begin{array}{c}\mathrm{MG} \\
\mathrm{M} \\
\mathrm{M} \\
\mathrm{PM}\end{array}$ & $\begin{array}{l}\text { A } \\
\text { A } \\
\text { R } \\
\text { R }\end{array}$ & $\stackrel{R}{-}$ & $\overline{\mathrm{R}}$ & $\begin{array}{l}\mathrm{R} \\
\mathrm{F} \\
\mathrm{F} \\
\mathrm{R}\end{array}$ & $\begin{array}{l}- \\
-\end{array}$ & $\begin{array}{l}\text { R } \\
= \\
-\end{array}$ & & $\begin{array}{l}\mathrm{F} \\
\mathrm{R} \\
\mathrm{R} \\
-\end{array}$ & $\begin{array}{l}\mathrm{C} \\
\mathrm{F} \\
\mathrm{F} \\
-\end{array}$ & $\begin{array}{l}\mathrm{F} \\
\mathrm{R} \\
\mathrm{F}\end{array}$ & $\begin{array}{l}\mathrm{C} \\
\mathrm{C} \\
\mathrm{C} \\
\mathrm{C}\end{array}$ & $\begin{array}{l}R \\
= \\
-\end{array}$ & $\begin{array}{l}\bar{z} \\
\bar{z}\end{array}$ & $\bar{z}$ & $\begin{array}{l}\mathrm{R} \\
\mathrm{R} \\
+\end{array}$ & $\begin{array}{l}\mathrm{c} \\
\frac{\mathrm{t}}{+} \\
+\end{array}$ & - & $\begin{array}{cc}\mathrm{R} & \mathrm{F} \\
+ & \mathrm{F} \\
& \mathrm{F} \\
? & \mathrm{~F}\end{array}$ & $\begin{array}{ll}F & R \\
F & - \\
F & -\end{array}$ & 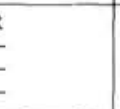 & & $\begin{array}{ll}F & R \\
R & + \\
R & \pm \\
R & -\end{array}$ & $\frac{F}{F}$ & $\begin{array}{l}\mathrm{R} \\
+ \\
? \\
?\end{array}$ \\
\hline
\end{tabular}

Note: $\mathrm{G}=$ good; $\mathrm{m}=$ moderate $\mathrm{P}=$ poor $; \mathrm{PM}=$ poor to moderate; $-=$ looked for but not found; $+=$ one specimen; $\mathrm{A}=$ abundant $\mathrm{C}=$ common; $\mathrm{F}=$ few; $\mathrm{R}=$ rare; ? = uncertain identification. 
Table 1. (Continued).

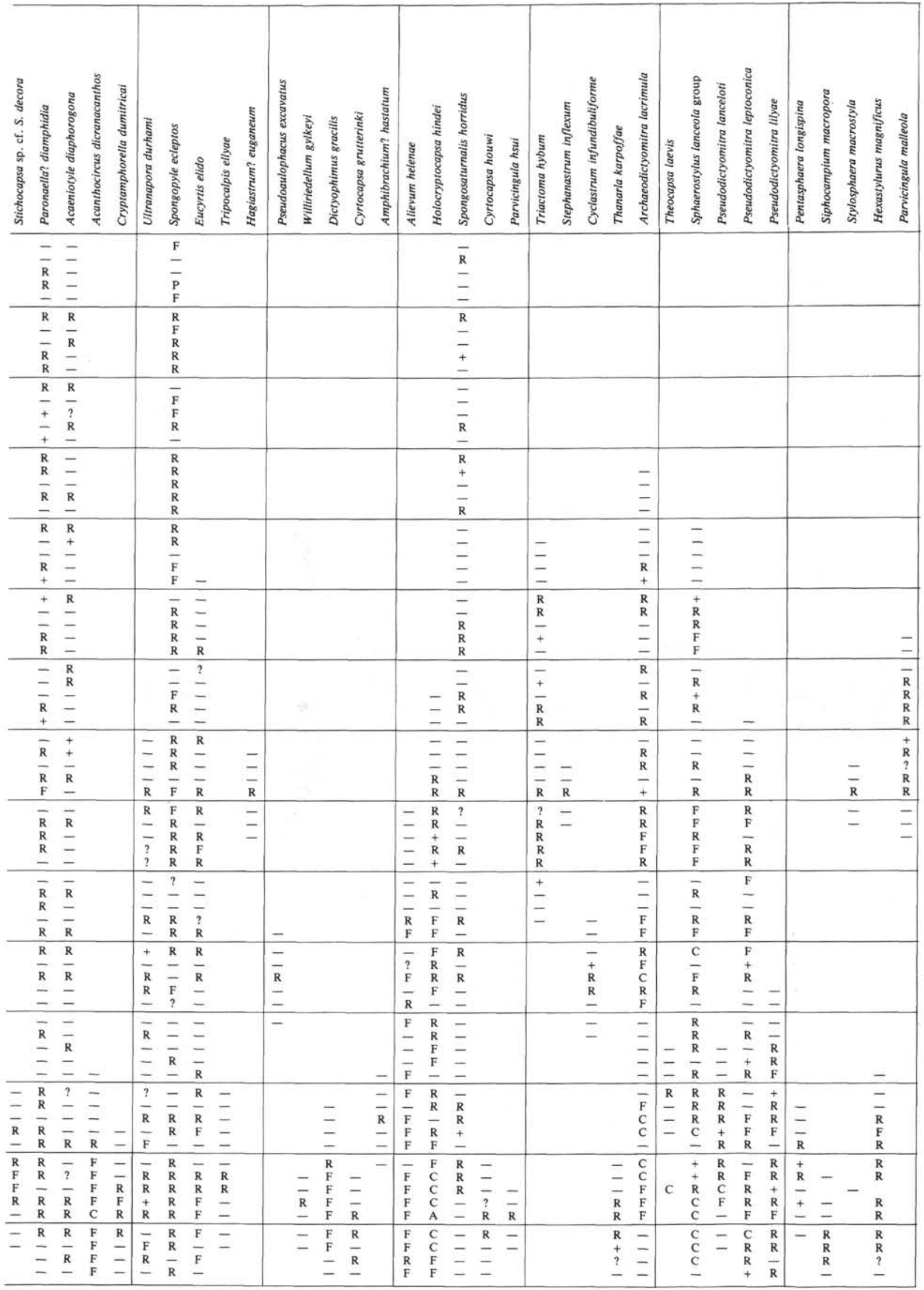




\section{A. $\mathrm{SCHAAF}$}

Table 1. (Continued).

\begin{tabular}{|c|c|c|c|c|c|c|c|c|c|c|c|c|c|c|c|c|c|c|c|c|c|c|c|c|c|c|c|c|}
\hline $\begin{array}{c}\text { Sample } \\
\text { (interval in } \mathrm{cm} \text { ) }\end{array}$ & & & 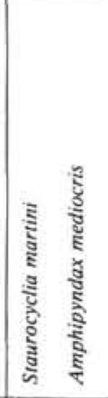 & 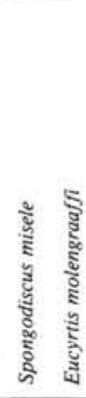 & 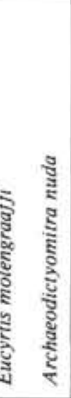 & 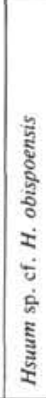 & 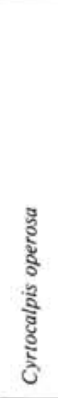 & 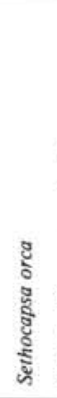 & 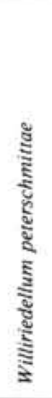 & 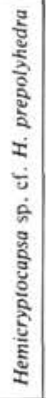 & 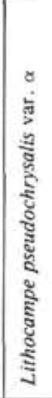 & 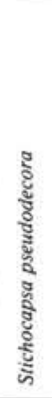 & 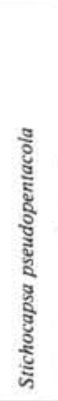 & 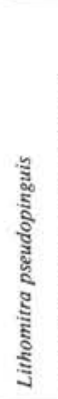 & 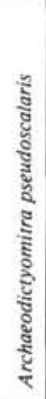 & 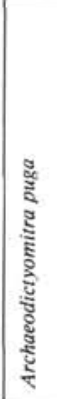 & $\begin{array}{l}\text { हू } \\
\text { בิ } \\
\text { है } \\
\text { हूँ }\end{array}$ & हूँ & 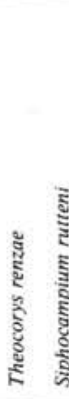 & हू & 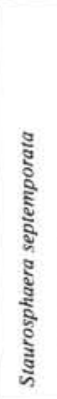 & $\begin{array}{l}\text { हू } \\
\text { है } \\
\text { हू } \\
\text { है } \\
\text { है }\end{array}$ & 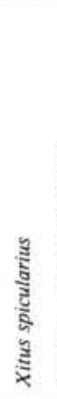 & 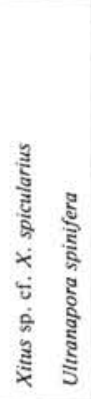 & 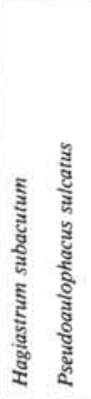 & 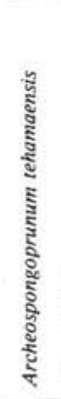 & 这 & 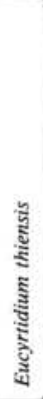 \\
\hline $\begin{array}{l}463-52, \mathrm{CC} \\
53-1,130-131 \\
56-1,27-28 \\
58-2,125-126 \\
59-2,10-11 \\
\end{array}$ & $\begin{array}{l}\mathrm{F} \\
\mathrm{F} \\
\mathrm{C} \\
\mathrm{C} \\
\mathrm{C} \\
\end{array}$ & \begin{tabular}{|c|}
$P$ \\
$P M$ \\
$P M$ \\
$P$ \\
$P M$ \\
\end{tabular} & & & & & & & & & & & & & & & & & & & & $\begin{array}{l}\bar{z} \\
\bar{z} \\
\bar{z}\end{array}$ & $\begin{array}{l}\bar{R} \\
\bar{R} \\
\mathrm{R}\end{array}$ & & & & $\begin{array}{l}\bar{z} \\
\bar{z}\end{array}$ & \\
\hline $\begin{array}{l}60-2,42-43 \\
61-1,29-30 \\
62-1,53-54 \\
62-1,91-92 \\
62-1,148-149 \\
\end{array}$ & $\begin{array}{l}\mathrm{A} \\
\mathrm{A} \\
\mathrm{A} \\
\mathrm{A} \\
\mathrm{A}\end{array}$ & \begin{tabular}{|l|}
$\mathrm{M}$ \\
$\mathrm{M}$ \\
$\mathrm{M}$ \\
$\mathrm{M}$ \\
$\mathrm{M}$ \\
\end{tabular} & & & & & & & & & & & & & & & & & & & & $\begin{array}{l}\text { R } \\
? \\
- \\
\\
+\end{array}$ & $\begin{array}{l}- \\
\bar{R} \\
R \\
R\end{array}$ & & & & $\begin{array}{l}\mathrm{R} \\
+ \\
\frac{+}{?} \\
-\end{array}$ & \\
\hline $\begin{array}{l}62-2,15-16 \\
62-2,119-120 \\
63, C C \\
64-1,66-67 \\
64-2,31-32\end{array}$ & $\begin{array}{l}A \\
A \\
A \\
A \\
A\end{array}$ & \begin{tabular}{|c|}
$M$ \\
$M$ \\
$M$ \\
$P M$ \\
$P M$ \\
\end{tabular} & & & & & & & & & & & & & & & & & & & & $\begin{array}{l}\bar{R} \\
\mathrm{R} \\
\overline{-} \\
\end{array}$ & $\frac{\mathrm{R}}{\mathrm{R}}$ & & & & $\begin{array}{l}\mathrm{R} \\
\mathrm{R} \\
+ \\
\mathrm{R} \\
- \\
\end{array}$ & \\
\hline $\begin{array}{l}64-3,31-32 \\
65-2,9-10 \\
66-1,27-28 \\
66-2,27-28 \\
66-3,21-22 \\
\end{array}$ & $\begin{array}{l}\mathrm{A} \\
\mathrm{A} \\
\mathrm{A} \\
\mathrm{A} \\
\mathrm{C}\end{array}$ & \begin{tabular}{|l|} 
PM \\
PM \\
PM \\
PM \\
M \\
\end{tabular} & & & & & & & & & & & & & & & & & & & & $\begin{array}{l}R \\
+ \\
\\
+ \\
-\end{array}$ & $\begin{array}{l}\mathrm{R} \\
\mathrm{R} \\
\mathrm{R} \\
\mathrm{R} \\
\end{array}$ & & & & $\begin{array}{l}\overline{\mathrm{R}} \\
? \\
\mathrm{R} \\
\end{array}$ & \\
\hline $\begin{array}{l}67-1,6-7 \\
67-2,110-111 \\
69-2,90-91 \\
69, C C \\
70-1,35-36 \\
\end{array}$ & \begin{tabular}{l|}
$\mathrm{C}$ \\
$\mathrm{A}$ \\
$\mathrm{R}$ \\
$\mathrm{C}$ \\
$\mathrm{C}$ \\
\end{tabular} & \begin{tabular}{|l|}
$\mathrm{P}$ \\
$\mathrm{P}$ \\
$\mathrm{P}$ \\
$\mathrm{P}$ \\
$\mathrm{P}$ \\
\end{tabular} & & & & & & - & & & & & & & & & & & & & & $\begin{array}{l}\overline{\mathrm{R}} \\
\bar{?} \\
+ \\
\end{array}$ & $\begin{array}{l}\mathrm{R} \\
\overline{-} \\
\overline{\mathrm{F}}\end{array}$ & & & & $\begin{array}{l}\mathrm{R} \\
? \\
\\
\mathrm{R} \\
\mathrm{R} \\
\end{array}$ & \\
\hline $\begin{array}{l}70-5,28-29 \\
71-2,99-100 \\
71-3,54-55 \\
72-1,43-44 \\
72-3,14-15\end{array}$ & \begin{tabular}{l|}
$\mathrm{F}$ \\
$\mathrm{A}$ \\
$\mathrm{A}$ \\
$\mathrm{A}$ \\
$\mathrm{A}$ \\
\end{tabular} & \begin{tabular}{|c|}
$\mathrm{P}$ \\
$\mathrm{PM}$ \\
$\mathrm{M}$ \\
$\mathrm{M}$ \\
$\mathrm{M}$ \\
\end{tabular} & $=$ & $\bar{z}$ & & & & $\begin{array}{l}- \\
\overline{-} \\
\bar{R} \\
\mathrm{R}\end{array}$ & & & & & & & & & & = & & & & $\begin{array}{l}\overline{\mathrm{R}} \\
\overline{\mathrm{R}} \\
?\end{array}$ & $\begin{array}{l}\mathrm{F} \\
\mathrm{R} \\
\mathrm{F} \\
\mathrm{F} \\
\mathrm{F} \\
\end{array}$ & & & & $\begin{array}{l}R \\
R \\
F \\
R \\
\end{array}$ & \\
\hline $\begin{array}{l}72-5,20-21 \\
72, C C \\
73-1,18-19 \\
73-1,140-141 \\
73-2,18-19\end{array}$ & \begin{tabular}{l|}
$\mathrm{F}$ \\
$\mathrm{C}$ \\
$\mathrm{A}$ \\
$\mathrm{C}$ \\
$\mathrm{F}$
\end{tabular} & \begin{tabular}{|c|}
$\mathrm{PM}$ \\
$\mathrm{M}$ \\
$\mathrm{M}$ \\
$\mathrm{P}$ \\
$\mathrm{P}$
\end{tabular} & $\begin{array}{l}= \\
= \\
=\end{array}$ & $\begin{array}{l}\mathrm{R} \\
\mathrm{R} \\
\mathrm{R} \\
- \\
-\end{array}$ & & & & $\begin{array}{l}\overline{\mathrm{R}} \\
? \\
\mathrm{R} \\
-\end{array}$ & & & & & & & & & & $\begin{array}{l}\bar{R} \\
\mathrm{R} \\
- \\
-\end{array}$ & & & & $\frac{\bar{R}}{\bar{R}}$ & $\begin{array}{l}\frac{\mathrm{R}}{\mathrm{R}} \\
\frac{-}{-}\end{array}$ & & & & $\begin{array}{l}\bar{R} \\
\mathrm{~F} \\
\mathrm{R} \\
\end{array}$ & \\
\hline $\begin{array}{l}73-3,19-20 \\
73-4,10-11 \\
73-4,116-117 \\
74-1,110-111 \\
75-1,21-22\end{array}$ & \begin{tabular}{|c|}
$\mathrm{F}$ \\
$\mathrm{F}$ \\
$\mathrm{F}$ \\
$\mathrm{F}$ \\
$\mathrm{A}$
\end{tabular} & \begin{tabular}{|c|}
$\mathrm{P}$ \\
$\mathrm{PM}$ \\
$\mathrm{M}$ \\
$\mathrm{M}$ \\
$\mathrm{M}$ \\
\end{tabular} & \begin{tabular}{|ll}
- & $\bar{R}$ \\
$\bar{R}$ & $R$ \\
$R$ & $\bar{R}$ \\
$R$ & $F$ \\
\end{tabular} & $\begin{array}{l}\mathrm{R} \\
? \\
\mathrm{R} \\
? \\
\mathrm{R}\end{array}$ & & & & $\begin{array}{l}\mathrm{R} \\
\overline{-} \\
\overline{+} \\
\mathrm{R}\end{array}$ & $\bar{z}$ & & & & & & & & & $\begin{array}{l}? \\
R \\
R \\
R \\
F\end{array}$ & & & & $\begin{array}{l}\bar{R} \\
\bar{R} \\
R\end{array}$ & $\begin{array}{l}\mathrm{R} \\
\frac{\mathrm{R}}{\mathrm{R}} \\
\mathrm{R}\end{array}$ & & $\begin{array}{l}\overline{\bar{z}} \\
\overline{\bar{R}} \\
\overline{\mathrm{R}}\end{array}$ & & $\begin{array}{l}\overline{-} \\
\bar{F} \\
\bar{F}\end{array}$ & \\
\hline $\begin{array}{l}76, \mathrm{CC} \\
77-1,7-8 \\
77-1,60-61 \\
78-1,72-73 \\
78-1,111-112 \\
\end{array}$ & \begin{tabular}{l|}
$\mathrm{C}$ \\
$\mathrm{A}$ \\
$\mathrm{C}$ \\
$\mathrm{A}$ \\
$\mathrm{A}$ \\
\end{tabular} & \begin{tabular}{|c|}
$\mathrm{P}$ \\
$\mathrm{P}$ \\
$\mathrm{PM}$ \\
$\mathrm{PM}$ \\
$\mathrm{PM}$ \\
\end{tabular} & \begin{tabular}{|ll}
- & $R$ \\
- & + \\
- & $R$ \\
& $F$ \\
& $F$ \\
\end{tabular} & $\bar{z}$ & & & & $\begin{array}{l}? \\
\mathrm{R} \\
\mathrm{F} \\
\mathrm{F} \\
\mathrm{R}\end{array}$ & $\begin{array}{l}-\overline{1} \\
\bar{R} \\
\mathrm{R} \\
?\end{array}$ & & & & & & & & & $\begin{array}{l}\bar{F} \\
\mathrm{R} \\
\mathrm{R} \\
\end{array}$ & & & & $\begin{array}{l}\bar{z} \\
\bar{z}\end{array}$ & $\begin{array}{l}\mathrm{R} \\
\mathrm{F} \\
\mathrm{F} \\
\mathrm{R} \\
\mathrm{R} \\
\end{array}$ & & $\begin{array}{l}\bar{z} \\
\overline{-}\end{array}$ & & $\begin{array}{l}R \\
R \\
R \\
F \\
F \\
\end{array}$ & \\
\hline $\begin{array}{l}79-1,36-37 \\
80-1,59-60 \\
81-2,45-46 \\
82, \mathrm{CC} \\
83-1,0-1\end{array}$ & \begin{tabular}{|l|}
$\mathrm{R}$ \\
$\mathrm{R}$ \\
$\mathrm{R}$ \\
$\mathrm{A}$ \\
$\mathrm{A}$ \\
\end{tabular} & \begin{tabular}{|c|}
$\mathrm{P}$ \\
$\mathrm{P}$ \\
$\mathrm{P}$ \\
$\mathrm{M}$ \\
$\mathrm{M}$ \\
\end{tabular} & $\begin{array}{l}- \\
+ \\
? \\
\mathrm{R} \\
\mathrm{F}\end{array}$ & & & & & $\begin{array}{l}\overline{-} \\
\overline{\bar{R}} \\
\mathrm{R}\end{array}$ & $\begin{array}{l} \pm \\
\bar{R} \\
R \\
R\end{array}$ & & & & & & & & & $\begin{array}{l}\mathrm{R} \\
\mathrm{R} \\
\mathrm{R} \\
? \\
\mathrm{P} \\
\mathrm{F}\end{array}$ & & & & & $\begin{array}{l}\overline{-} \\
\overline{\bar{F}} \\
\overline{\mathrm{F}} \\
\mathrm{R}\end{array}$ & & & $\begin{array}{l}\bar{z} \\
\bar{z}\end{array}$ & $\begin{array}{l}\bar{z} \\
\bar{F} \\
F\end{array}$ & \\
\hline $\begin{array}{l}83-1,38-39 \\
83-1,130-131 \\
84-1,5-6 \\
84-1,19-20 \\
84, C C\end{array}$ & \begin{tabular}{|l|}
$\mathrm{A}$ \\
$\mathrm{R}$ \\
$\mathrm{A}$ \\
$\mathrm{F}$ \\
$\mathrm{F}$ \\
\end{tabular} & \begin{tabular}{|c|}
$\mathrm{M}$ \\
$\mathrm{M}$ \\
$\mathrm{M}$ \\
$\mathrm{M}$ \\
$\mathrm{PM}$ \\
\end{tabular} & $\begin{array}{l}\mathrm{F} \\
\mathrm{F} \\
\mathrm{F} \\
\overline{\mathrm{R}}\end{array}$ & & & & & $\begin{array}{l}\mathrm{R} \\
+ \\
\mathrm{R} \\
\mathrm{R}\end{array}$ & $\begin{array}{l}F \\
R \\
F \\
\end{array}$ & $\begin{array}{l}\bar{z} \\
\bar{z}\end{array}$ & & $\begin{array}{l}- \\
\bar{R} \\
\bar{R}\end{array}$ & & & & & $\begin{array}{l}\bar{z} \\
\bar{R} \\
\overline{-}\end{array}$ & $\begin{array}{l}\mathrm{R} \\
\mathrm{F} \\
\mathrm{R} \\
-\end{array}$ & $\begin{array}{l}\bar{z} \\
\bar{R}\end{array}$ & & & & $\begin{array}{l}\frac{\mathrm{R}}{\mathrm{R}} \\
\frac{-}{-}\end{array}$ & & & $\begin{array}{l}R \\
R \\
+ \\
\bar{R}\end{array}$ & $\begin{array}{l}\mathrm{F} \\
\bar{F} \\
\mathrm{R} \\
-\end{array}$ & \\
\hline $\begin{array}{l}85-1,19-20 \\
85-1,142-143 \\
85-2,20-21 \\
86-1,31-32 \\
86-1,115-116\end{array}$ & $\begin{array}{l}F \\
F \\
F \\
F \\
F\end{array}$ & \begin{tabular}{|c|}
$\mathrm{P}$ \\
$\mathrm{PM}$ \\
$\mathrm{M}$ \\
$\mathrm{P}$ \\
$\mathrm{M}$ \\
\end{tabular} & $\begin{array}{l}? \\
\mathrm{R} \\
\mathrm{R}\end{array}$ & & & - & $\mathrm{R}$ & $\begin{array}{l}\bar{z} \\
\bar{z}\end{array}$ & $\begin{array}{l}\mathrm{R} \\
\overline{\mathrm{R}} \\
\mathrm{R}\end{array}$ & \begin{tabular}{l|}
$R$ \\
+ \\
- \\
-
\end{tabular} & & $\begin{array}{l}- \\
\bar{R} \\
\overline{-}\end{array}$ & & ? & - & $\begin{array}{l}\mathrm{R} \\
\mathrm{R}\end{array}$ & $\overline{-}$ & $\mathrm{R}$ & $\begin{array}{l}\mathrm{F} \\
\mathrm{R}\end{array}$ & & $\bar{z}$ & & $\frac{\bar{R}}{\bar{R}}$ & & & $\begin{array}{l}\overline{-} \\
\bar{R} \\
\underline{-}\end{array}$ & $\begin{array}{l}\bar{R} \\
- \\
-\end{array}$ & \\
\hline $\begin{array}{l}86, \mathrm{CC} \\
87-1,6-7 \\
87-1,65-66 \\
88-1,0-1 \\
88-1,29-30\end{array}$ & \begin{tabular}{|l|} 
F \\
F \\
C \\
A \\
F
\end{tabular} & \begin{tabular}{|c|}
$\mathrm{M}$ \\
$\mathrm{PM}$ \\
$\mathrm{M}$ \\
$\mathrm{PM}$ \\
$\mathrm{P}$ \\
\end{tabular} & $\begin{array}{l}\mathrm{R} \\
\mathrm{F} \\
\mathrm{C} \\
\mathrm{R}\end{array}$ & & - & & $=$ & & $\begin{array}{l}? \\
\text { F } \\
\text { F } \\
-\end{array}$ & \begin{tabular}{l|}
- \\
$R$ \\
$?$ \\
- \\
\end{tabular} & - & $\begin{array}{l}R \\
R \\
F \\
F \\
R\end{array}$ & $\bar{z}$ & & $\begin{array}{l}- \\
R \\
F \\
C \\
R\end{array}$ & $\overline{-}$ & $\begin{array}{l}\overline{-} \\
\bar{R} \\
+ \\
-\end{array}$ & & $\begin{array}{l}\mathrm{F} \\
\mathrm{R} \\
\mathrm{F} \\
\mathrm{C} \\
\mathrm{F}\end{array}$ & & $\begin{array}{l}\bar{Z} \\
\bar{R} \\
\mathrm{R} \\
\mathrm{R}\end{array}$ & & $\begin{array}{l}\mathrm{R} \\
\mathrm{R} \\
\mathrm{R} \\
-\end{array}$ & & & $\begin{array}{l}\bar{R} \\
R \\
R \\
- \\
\end{array}$ & $\begin{array}{l}\mathrm{R} \\
\mathrm{R} \\
\mathrm{F} \\
-\end{array}$ & \\
\hline $\begin{array}{l}88-1,52-53 \\
89-1,15-16 \\
89-1,23-24 \\
89-1,94-95 \\
89-1,105-106 \\
\end{array}$ & \begin{tabular}{|l|}
$\mathrm{F}$ \\
$\mathrm{C}$ \\
$\mathrm{A}$ \\
$\mathrm{C}$ \\
$\mathrm{A}$ \\
\end{tabular} & \begin{tabular}{|c|}
$\mathrm{M}$ \\
$\mathrm{M}$ \\
$\mathrm{M}$ \\
$\mathrm{MG}$ \\
$\mathrm{G}$ \\
\end{tabular} & $\begin{array}{ll}\mathrm{F} \\
\mathrm{R} \\
\mathrm{F} \\
\mathrm{R} \\
\end{array}$ & $-\frac{\bar{R}}{R}$ & $\begin{array}{l}- \\
\overline{-} \\
\bar{R} \\
\mathrm{~F}\end{array}$ & $\frac{?}{-}$ & $\begin{array}{l}\bar{R} \\
R \\
R \\
\end{array}$ & $\mathrm{~F}$ & $\begin{array}{l}\mathrm{R} \\
\mathrm{R} \\
+ \\
\mathrm{F} \\
\mathrm{F} \\
\end{array}$ & $\begin{array}{l}\bar{R} \\
\bar{R} \\
\mathrm{R}\end{array}$ & $\begin{array}{l}\overline{-} \\
\bar{F} \\
R \\
R \\
\end{array}$ & $\begin{array}{l}- \\
+ \\
+ \\
+ \\
F\end{array}$ & $\begin{array}{l}\mathrm{R} \\
\mathrm{R} \\
- \\
= \\
-\end{array}$ & $\begin{array}{l}\mathrm{F} \\
\mathrm{R} \\
\mathrm{R} \\
\end{array}$ & $\begin{array}{l}R \\
\overline{-} \\
\bar{F}\end{array}$ & $\begin{array}{l}\bar{R} \\
+ \\
R \\
R \\
\end{array}$ & $\begin{array}{l}\bar{R} \\
\overline{-}\end{array}$ & $\mathrm{c}$ & $\begin{array}{l}\mathrm{F} \\
\mathrm{C} \\
\mathrm{F} \\
\mathrm{C}\end{array}$ & $\begin{array}{l}- \\
-\end{array}$ & $\begin{array}{l}\mathrm{R} \\
\mathrm{F} \\
\mathrm{F} \\
\mathrm{C} \\
\mathrm{C}\end{array}$ & & $\begin{array}{l}\mathrm{R} \\
\mathrm{R} \\
\mathrm{R} \\
\mathrm{R} \\
\mathrm{F} \\
\end{array}$ & $\begin{array}{l}\bar{y} \\
\bar{R}=\end{array}$ & & $\begin{array}{l} \\
+ \\
F \\
R \\
F \\
\end{array}$ & $\begin{array}{l}\mathrm{R} \\
\mathrm{R} \\
\mathrm{R} \\
- \\
- \\
\end{array}$ & $\begin{array}{l}- \\
= \\
-\end{array}$ \\
\hline $\begin{array}{l}90, \mathrm{CC} \\
91, \mathrm{CC} \\
92-1,0-1 \\
92-1,10-11\end{array}$ & \begin{tabular}{|l} 
A \\
C \\
F \\
F
\end{tabular} & \begin{tabular}{c|}
$\mathrm{MG}$ \\
$\mathrm{M}$ \\
$\mathrm{M}$ \\
$\mathrm{PM}$
\end{tabular} & $\begin{array}{l}\bar{z} \\
\bar{z}\end{array}$ & $-\bar{R}$ & $\begin{array}{l}\mathrm{R} \\
+ \\
- \\
-\end{array}$ & $\begin{array}{l}R \\
R \\
+ \\
-\end{array}$ & $\begin{array}{l}\mathrm{F} \\
\mathrm{R}\end{array}$ & $\underline{\mathrm{F}}$ & $\begin{array}{l}\mathrm{C} \\
+ \\
\mathrm{R} \\
\mathrm{F}\end{array}$ & $\begin{array}{l}\mathrm{R} \\
\mathrm{R} \\
\mathrm{R} \\
-\end{array}$ & $\begin{array}{l}\mathrm{R} \\
\mathrm{R} \\
\mathrm{R} \\
\\
\end{array}$ & $\begin{array}{l}\mathrm{F} \\
\mathrm{R}\end{array}$ & $\stackrel{\mathrm{R}}{-}$ & $\begin{array}{l}+ \\
R \\
R \\
R\end{array}$ & $\begin{array}{l}F \\
+ \\
-\end{array}$ & $\frac{\bar{R}}{\bar{R}}$ & $\begin{array}{l}? \\
\mathrm{R}\end{array}$ & $\begin{array}{l}\mathrm{R} \\
\mathrm{R}\end{array}$ & $\begin{array}{l}\mathrm{C} \\
\mathrm{R}\end{array}$ & $\begin{array}{l}\mathrm{R} \\
- \\
-\end{array}$ & $\begin{array}{l}C \\
\mathrm{~F} \\
\mathrm{R} \\
\mathrm{R}\end{array}$ & & $\begin{array}{l}F \\
R \\
R \\
R\end{array}$ & $\begin{array}{l}=\quad F \\
=\quad R \\
=\quad R \\
R\end{array}$ & & $\begin{array}{l}\mathrm{F} \\
\mathrm{F} \\
-\end{array}$ & $\begin{array}{l}\bar{z} \\
\bar{z}\end{array}$ & $\begin{array}{l}\mathrm{R} \\
-\end{array}$ \\
\hline
\end{tabular}


Table 1. (Continued).

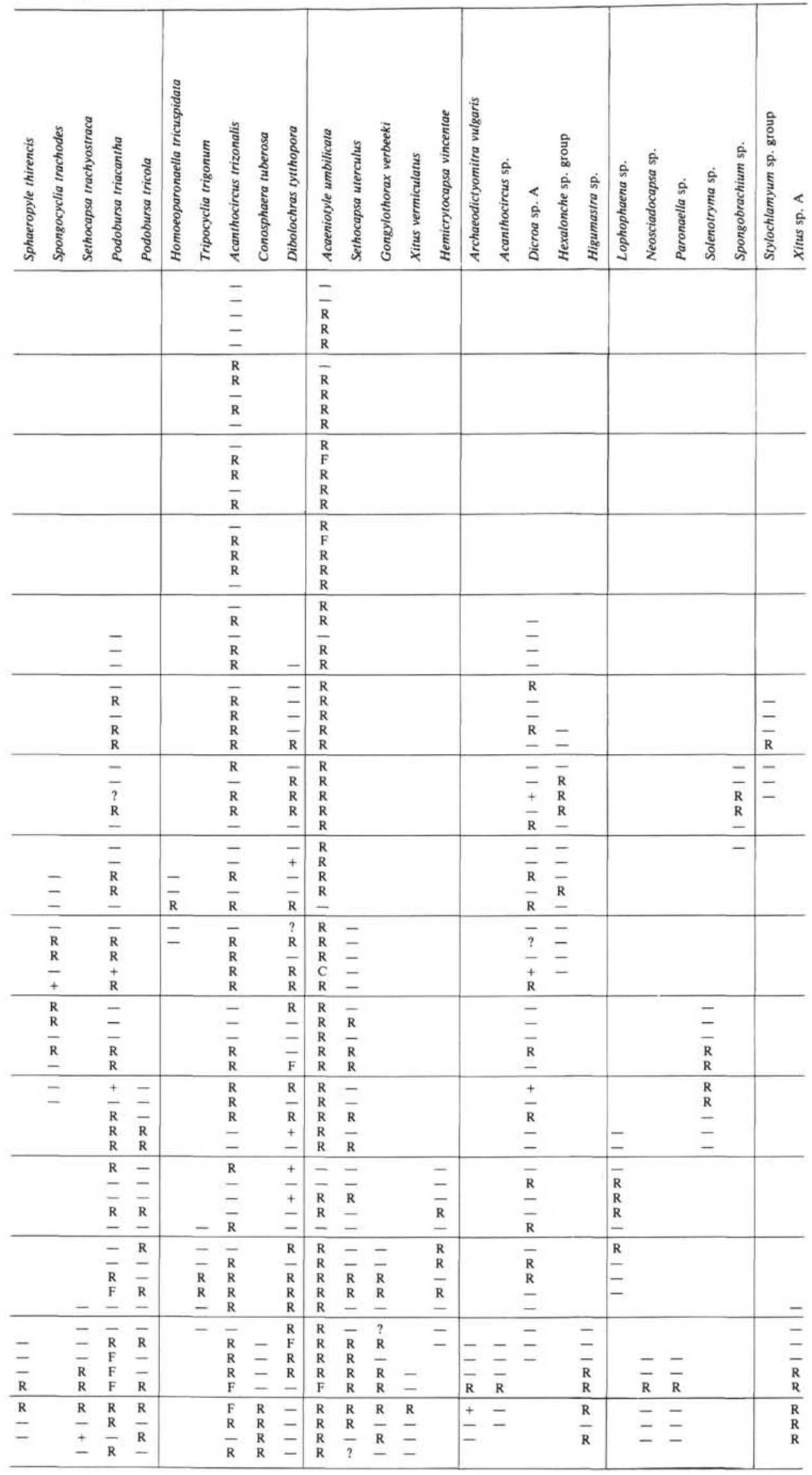


Table 2. Occurrence and abundance of late Early Cretaceous radiolarians of Site 464 (symbols as in Table 1).

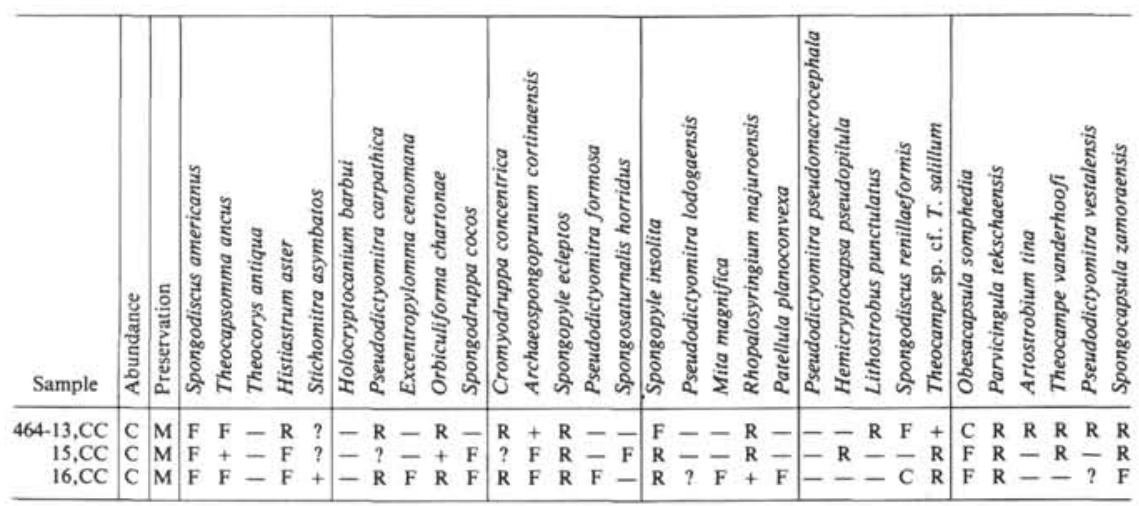

Table 3. Occurrence and abundance of late Early Cretaceous radiolarians of Site 465 (Hole 465A) (symbols as in Table 1).

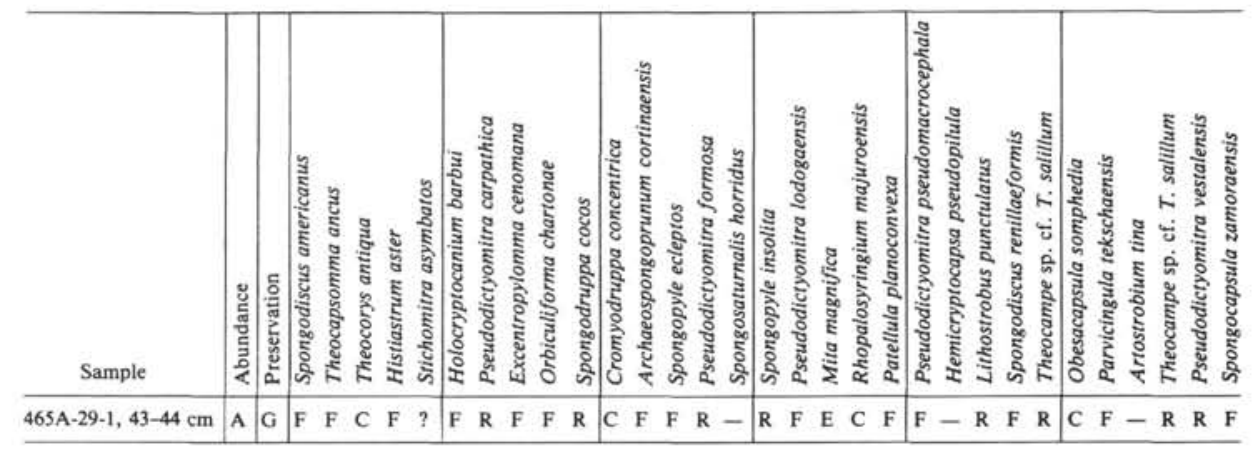

Table 4. Occurrence and abundance of late Early Cretaceous radiolarians of Site 466 (symbols as in Table 1).

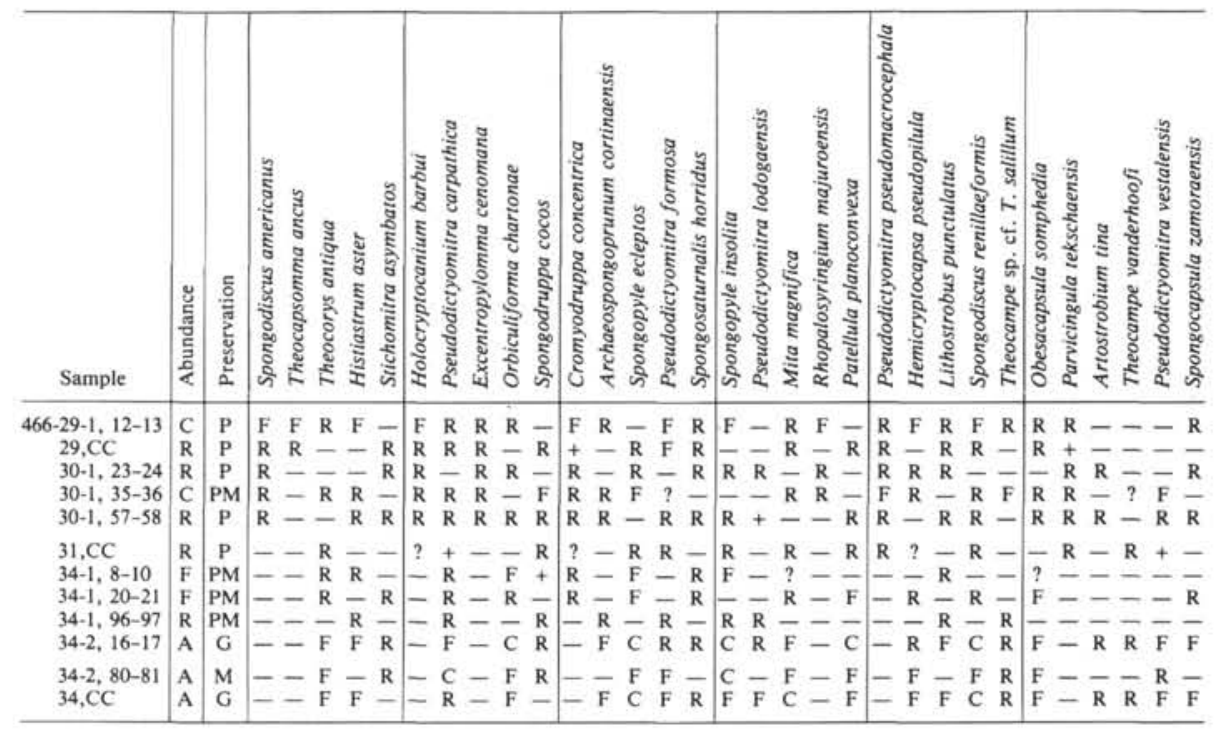

2) Nassellaria were subdivided according to the suprageneric classification of Petrushevskaya (1971).

3) Unless otherwise indicated, we have rather uncritically followed the generic assignments applied by earlier authors, since shortage of time has prevented examination of the relationships of type species of genera.

The outline is followed by an alphabetical listing of species encountered in this study. This replaces the traditional index. 
Table 5. Samples in which late Early Cretaceous radiolarians were not found.

\begin{tabular}{|c|c|c|c|}
\hline Site 463 & Site 464 & $\begin{array}{c}\text { Site 465 } \\
\text { (Hole 465A) }\end{array}$ & Site 466 \\
\hline up to 55 and & $11-1,66-67$ & $26-1,20-21$ & $29-2,20-21$ \\
\hline $55-1,42-43$ & $11-1,82-83$ & $26-1,34-35$ & $35-1,21-22$ \\
\hline $55, \mathrm{CC}$ & $12-1,22-23$ & $26-1,51-52$ & $35-1,57-58$ \\
\hline $57-1,19-20$ & $12-1,81-82$ & $26-1,116-117$ & $35, \mathrm{CC}$ \\
\hline $57-1,110-111$ & $12-2,12-14$ & $27-1,66-67$ & \\
\hline $57-2,17-18$ & $13-1,12-14$ & $27-1,100-101$ & \\
\hline $58-1,96-97$ & $17, \mathrm{CC}$ & $27-2,1-2$ & \\
\hline $58-3,20-21$ & $18, \mathrm{CC}$ & $27-2,111-112$ & \\
\hline $58, \mathrm{CC}$ & $19, \mathrm{CC}$ & $27-2,149-150$ & \\
\hline $59-1,118-119$ & $21-1,3-4$ & $27, \mathrm{CC}$ & \\
\hline $59-3,15-16$ & $23-1,48-49$ & $28-1,27-28$ & \\
\hline $59-3,138-139$ & $24, C C$ & $28-1,57-58$ & \\
\hline $59, \mathrm{CC}$ & $26-1,90-91$ & $28-1,130-131$ & \\
\hline $60-1,29-30$ & $26 . \mathrm{CC}$ & $28-2,2-3$ & \\
\hline $60-1,145-146$ & $27-1,30-31$ & $28, C C$ & \\
\hline $60-2,121-122$ & $27, \mathrm{CC}$ & $29-2,45-46$ & \\
\hline $60-3,32-33$ & $28, \mathrm{CC}$ & $30-1,32-33$ & \\
\hline $60-3,88-89$ & $28-1,30-31$ & $31-1,19-20$ & \\
\hline $60-4,4-5$ & $29 . C \mathrm{C}$ & $33-1,31-32$ & \\
\hline $61-1,110-111$ & $30-1,27-29$ & $33-2,86-87$ & \\
\hline $62-3,18-19$ & $30-1,46-47$ & $34-1,52-53$ & \\
\hline $62-3,59-60$ & $32-1,18-19$ & $34-2,29-30$ & \\
\hline $63-1,109-110$ & $33-1,30-31$ & $35-1,12-13$ & \\
\hline $63-2,53-54$ & $34 . C C$ & $36-1,62-63$ & \\
\hline $64, C \mathrm{C}$ & & $36-2,34-35$ & \\
\hline $65-1,11-12$ & & $37-1,38-39$ & \\
\hline $65-2,9-10$ & & $38-1,33-34$ & \\
\hline $65-3,8-9$ & & $38-2,63-64$ & \\
\hline $65, \mathrm{CC}$ & & $39-1,32-33$ & \\
\hline $68-1,21-22$ & & $40-1,25-26$ & \\
\hline $69-1,44-45$ & & $40-2,60-61$ & \\
\hline $70-6,21-22$ & & & \\
\hline $73-3,112-113$ & & & \\
\hline $76-1,69-70$ & & & \\
\hline
\end{tabular}

\section{Outline of Classification}

Subclass Radiolaria

Superorder Polycystina Ehrenberg, 1838, emend. Riedel, 1967a. Order Spumellaria Ehrenberg, 1875

Family Litheliidae Haeckel, 1862

Cromyodruppa concentrica Lipman, 1960

Family Actinommidae Haeckel, 1862, emend. Riedel, 1971

Acaeniotyle diaphorogona Foreman, 1973

Acaeniotyle umbilicata (Rüst, 1898)

Archaeospongoprunum cortinaensis Pessagno, 1973

Archaeospongoprunum tehamaensis Pessagno, 1973

Conosphaera tuberosa Tan Sin Hok, 1927

Dicroa sp. A Foreman, 1975

Hexalonche [?] sp. group

Hexastylurus magnificus Squinabol, 1904

Pentasphaera longispina Squinabol, 1904

?Sphaeropyle thirencis, $\mathrm{n} . \mathrm{sp}$.

Sphaerostylus lanceola (Parona, 1890)

Staurosphaera septemporata Parona, 1890

Stylosphaera macrostyla Rüst, 1888

Triactoma hybum Foreman, 1975

?Tripocyclia trigonum Rüst, 1885

Subfamily Saturnalinae Deflandre, 1953

Acanthocircus carinatus Foreman, 1973

Acanthocircus dicranacanthos (Squinabol, 1914)

Acanthocircus trizonalis (Rüst, 1898)

Acanthocircus sp.

Spongosaturnalis horridus (Squinabol, 1903) group

Family Porodiscidae Haeckel, 1881, emend. Petrushevskaya and Kozlova, 1972

Cyclastrum infundibuliforme Rüst, 1898

Hexinastrum cretaceum Lipman, 1960

Histiastrum aster Lipman, 1952

Staurocyclia martini Rüst, 1898

Stephanastrum inflexum Rüst, 1898
Family Hagiastridae Riedel, 1971, emend. Pessagno, 1971

Amphibrachium [?] hastatum Renz, 1974

Crucella sp. cf. C. cachensis Pessagno, 1971

Hagiastrum subacutum Rüst, 1885

Hagiastrum [?] euganeum (Squinabol, 1903)

Higumastra sp.

Homoeoparonaella tricuspidata (Rüst, 1898)

Paronaella [?] diamphidia Foreman, 1973

Paronaella sp.

Family Pseudoaulophacidae Riedel, 1967a

Alievium antiguum Pessasgno, 1972

Alievium helenae, n. sp.

Patellula planoconvexa (Pessagno, 1963)

Pseudoaulophacus [?] excavatus (Rüst, 1898)

Pseudoaulophacus [?] sulcatus (Rüst, 1898)

Family Spongodiscidae Haeckel, 1862, emend.

Petrushevskaya and Kozlova, 1972

Orbiculiforma chartonae n. sp.

Spongobrachium [?] sp.

Spongocyclia trachodes Renz, 1974

Spongodiscus americanus Kozlova, 1966

Spongodiscus misele, $\mathrm{n}$. $\mathrm{sp}$.

Spongodiscus renillaeformis Campbell and Clark, 1944

Spongodruppa cocos Rüst, 1898

Spongopyle ecleptos Renz, 1974

Spongopyle insolita Kozlova, 1966

Stylochlamyum [?] sp. group

Order Nassellaria Ehrenberg, 1875

Suborder Cyrtida Haeckel, 1862, emend. Petrushevskaya, 1971

Superfamily Eucyrtidioidea Ehrenberg, 1847, emend. Petrushevskaya, 1971

Family Eucyrtididae Ehrenberg, 1847, emend. Petrushevskaya, 1971

Archaeodictyomitra apiara (Rüst, 1885)

Archaeodictyomitra brouweri var. $\alpha$ (Tan Sin Hok, 1927)

Archaeodictyomitra lacrimula (Foreman, 1973)

Archaeodictyomitra nuda, n. sp.

Archaeodictyomitra pseudoscalaris (Tan Sin Hok, 1927)

Archaeodictyomitra puga, n. sp.

Archaeodictyomitra vulgaris Pessagno, 1977

Crolanium pythiae, n. sp.

Cyrtocapsa asseni var. $\alpha$ Tan Sin Hok, 1927

Cyrtocapsa grutterinki Tan Sin Hok, 1927

Cyrtocapsa houwi Tan Sin Hok, 1927

Cyrtocapsa molengraaffi Tan Sin Hok, 1927

?Diacanthocapsa boersmae, $\mathrm{n}$. sp.

Dibolachras tytthopora Foreman, 1973

Dictyophimus gracilis Tan Sin Hok, 1927

Eucyrtidium thiensis Tan Sin Hok, 1927

Eucyrtis sp. cf. E. bulbosa Renz, 1974

Eucyrtis columbaria Renz, 1974

Eucyrtis elido, n. sp.

Eucyrtis molengraaffi (Tan Sin Hok, 1927)

Eucyrtis tenuis (Rüst, 1885)

?Gongylothorax baumgartneri, $\mathrm{n}$. $\mathrm{sp}$.

?Gongylothorax verbeeki (Tan Sin Hok, 1927)

Hsuum sp. cf. H. obispoensis Pessagno, 1977

Lithocampe chenodes Renz, 1974 group

Lithocampe pseudochrysalis var. $\alpha$ Tan Sin Hok, 1927

Lithomitra [?] pseudopinguis Tan Sin Hok, 1927

Lithostrobus punctulatus Pessagno, 1963

Mita magnifica Pessagno, 1977

Neosciadocapsa sp.

Obesacapsula somphedia (Foreman, 1973)

Parvicingula sp. cf. $P$. altissima (Rüst, 1885) 


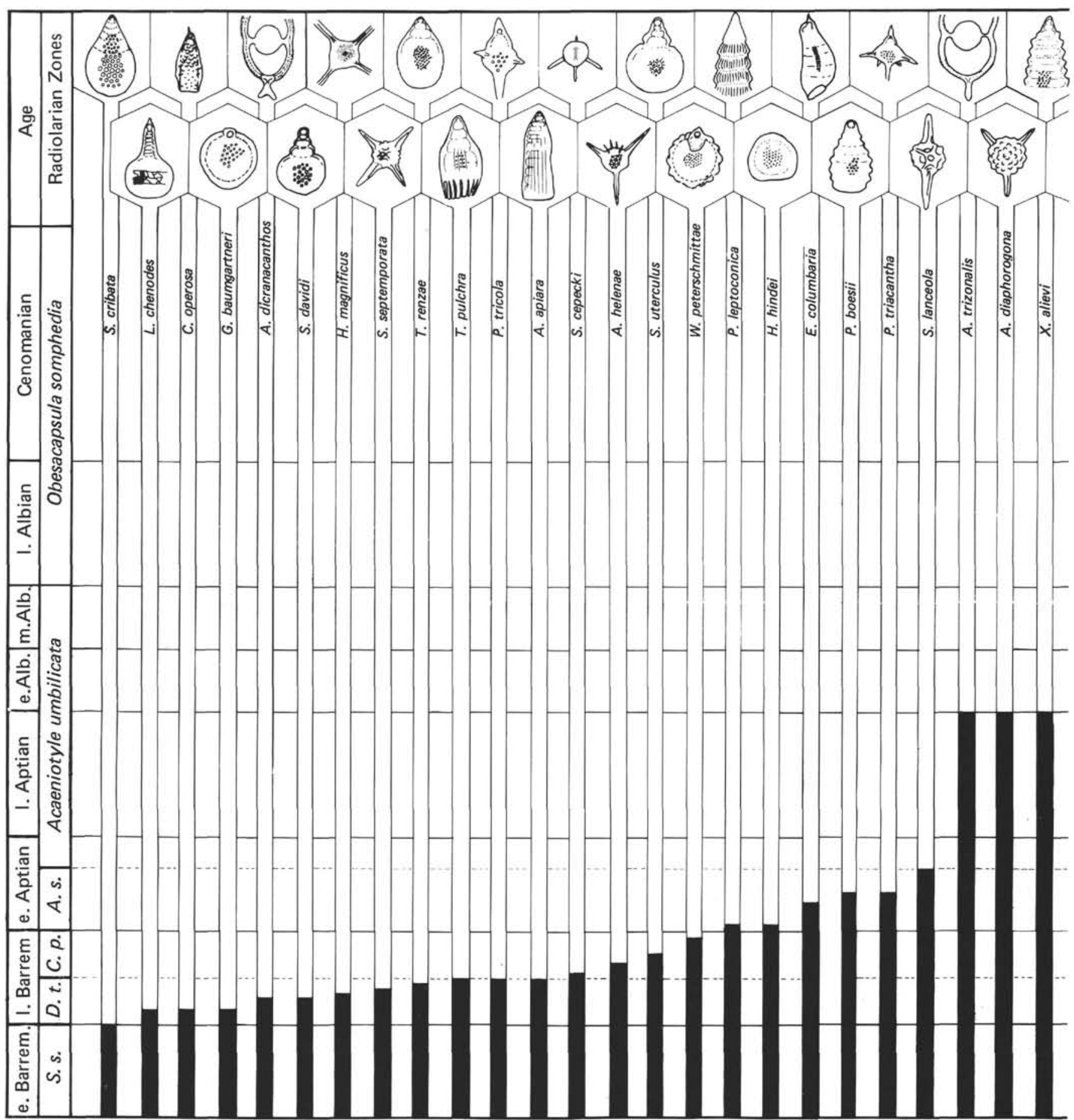

Figure 3. Range chart for late Early Cretaceous radiolarians.

Parvicingula boesii (Parona, 1890)

Parvicingula hsui Pessagno, 1977

Parvicingula malleola (Aliev, 1961) group

Parvicingula tekschaensis (Aliev, 1967)

Podubursa triacantha (Fischli, 1916)

Podubursa tricola Foreman, 1973

Pseudodictyomitra blabla, n. sp.

Pseudodictyomitra carpatica (Lozyniak, 1969)

Pseudodictyomitra formosa (Squinabol, 1904)
Pseudodictyomitra lanceloti, n. sp.

Pseudodictyomitra leptoconica (Foreman, 1973) group

Pseudodictyomitra lilyae (Tan Sin Hok, 1927)

Pseudodictyomitra lodogaensis Pessagno, 1977

Pseudodictyomitra vestalensis Pessagno, 1977

Pseudodictyomitra pseudomacrocephala (Squinabol, 1903) 


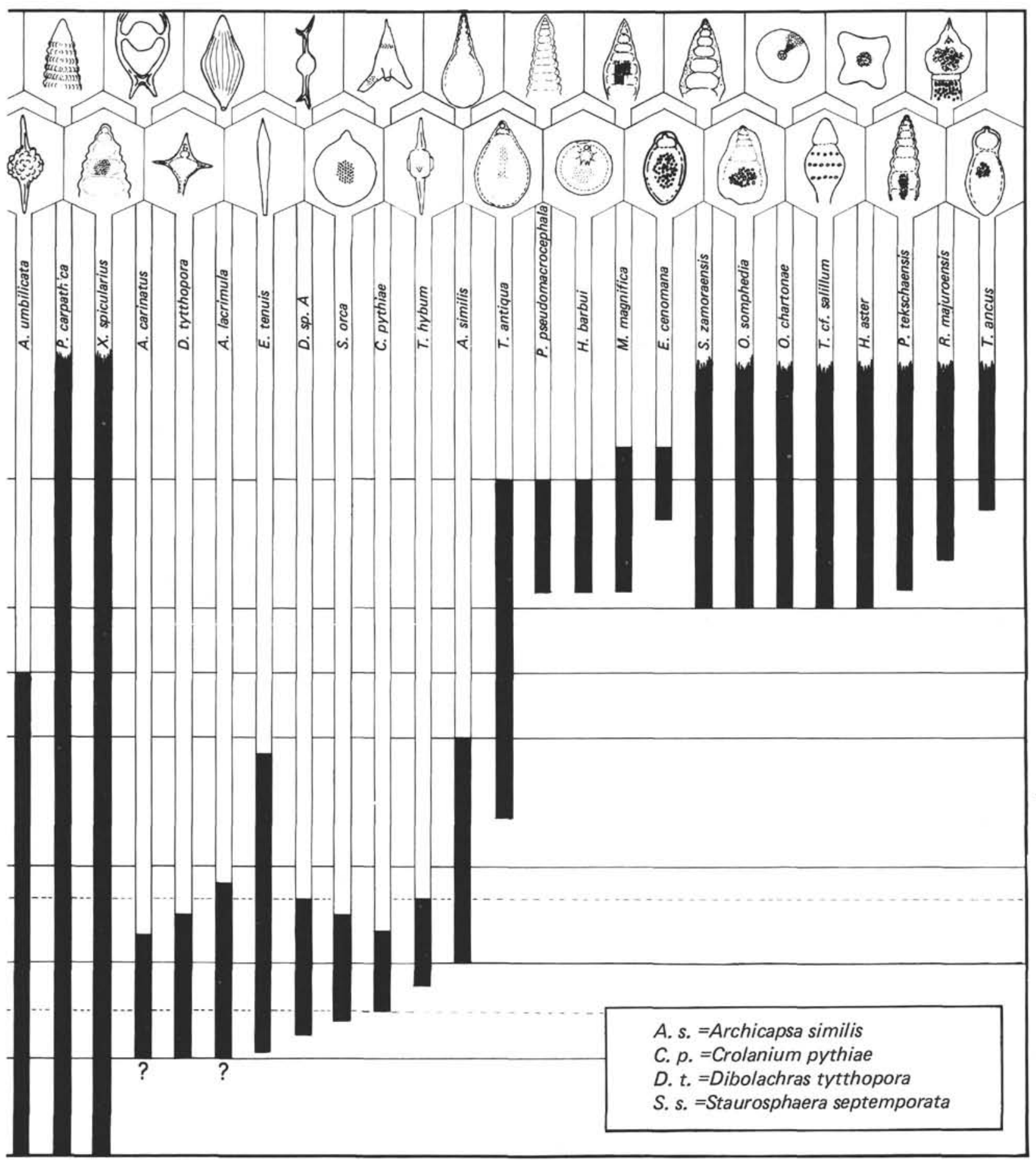

Figure 3. (Continued).

Saitoum cepeki, n. sp.

Sethocapsa orca Foreman, 1975

Sethocapsa trachyostraca Foreman, 1973

Sethocapsa uterculus (Parona, 1890)

Solenotryma sp.

Spongocapsula [?] zamoraensis Pessagno, 1976

Stichocapsa cribata Hinde, 1900

Stichocapsa decora Rüst, 1885

Stichocapsa sp. cf. S. decora Rüst, 1885
Stichocapsa pseudodecora Tan Sin Hok, 1927

Stichocapsa pseudopentacola Tan Sin Hok, 1927

Stichomitra asymbatos Foreman, 1968

Thanarla karpoffae, n. sp.

Thanarla pulchra (Squinabol, 1904)

Theocapsa laevis Tan Sin Hok, 1927

Theocapsomma ancus Foreman, 1968

Theocorys antiqua Squinabol, 1903 


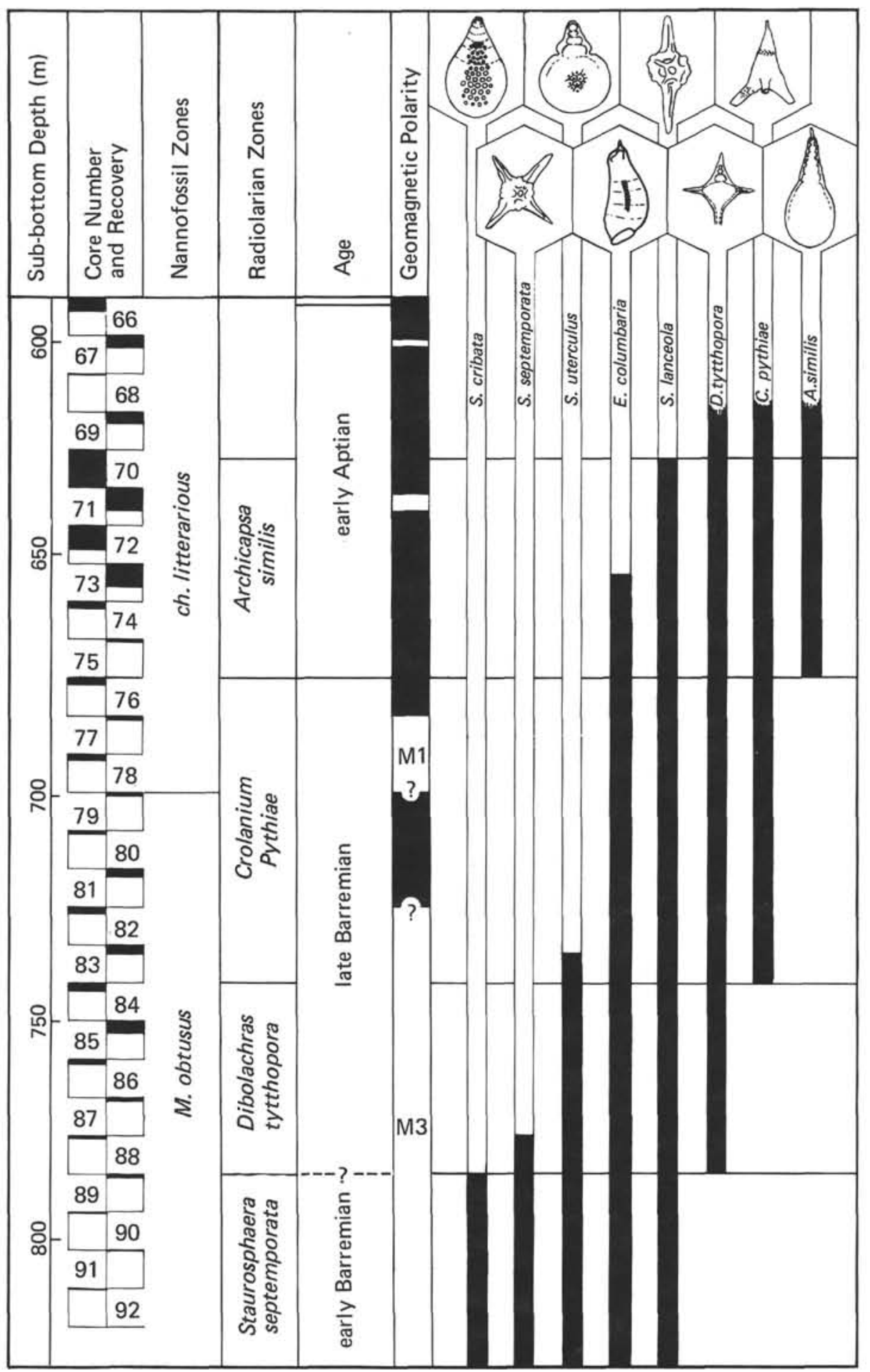

Figure 4. Relationship of the three new radiolarian zones to nannofossil zones and geomagnetic polarity.

Theocorys renzae, $\mathrm{n}$. sp.

Tripolcapis ellyae Tan Sin Hok, 1927

Ultranapora durhami Pessagno, 1977

Ultranapora spinifera Pessagno, 1977

Xitus alievi (Foreman, 1973)

Xitus spicularius (Aliev, 1961)

Xitus sp. cf. X. spicularius (Aliev, 1961)

Xitus vermiculatus (Renz, 1974)

Xitus sp. A
Subfamily Artostrobiinae Riedel, 1967b Artostrobium tina Foreman, 1971 Rhopalosyringium majuroensis $\mathrm{n} . \mathrm{sp}$. Theocampe sp. cf. T. salillum Foreman 1971 Theocampe vanderhoofi Campbell and Clark, 1944

Subfamily Plectopyramidinae Haecker, 1908, emend. Petrushevskaya, 1971

?Cyrtocalpis operosa Tan Sin Hok, 1927 
Subfamily Amphipyndacinae Riedel, 1967b, emend. Petrushevskaya, 1971 Amphipyndax mediocris (Tan Sin Hok, 1927) Siphocampium davidi, n. sp. Siphocampium macropora (Rüst, 1888) Siphocampium rutteni (Tan Sin Hok, 1927)

Family Williriedellidae Dumitrică, 1970

Cryptamphorella challengeri, $\mathrm{n}$. $\mathrm{sp}$. Cryptamphorella conara (Foreman, 1968) Cryptamphorella dumitricai, n. sp. Excentropylomma cenomana Dumitrică, 1970

Hemicryptocapsa sp. cf. H. cryptodon (Dumitrică, 1970)

Hemicryptocapsa sp. cf. $H$. prepolyhedra Dumitrică, 1970

Hemicryptocapsa pseudopilula Tan Sin Hok, 1927

Hemicryptocapsa vincentae, $\mathrm{n} . \mathrm{sp}$. Holocryptocanium barbui Dumitrică, 1970 Holocryptocapsa hindei Tan Sin Hok, 1927 Williriedellum carpathicum Dumitrică, 1970 Williriedellum gilkeyi Dumitrică, 1972 Williriedellum peterschmittae, n. sp.

Superfamily Plagiacanthoidea Hertwig, 1879, emend. Petrushevskaya, 1971

Family Plagiacanthidae Hertwig, 1879, emend. Petrushevskaya, 1971

Subfamily Lophophaeninae Haeckel, 1881, emend. Petrushevskaya, 1971 Archicapsa similis Parona, 1890 Lophophaena sp.

Taxonomic Listing

Acaeniotyle diaphorogona Foreman, 1973 (Pl. 15, Fig. 2)

Acaeniotyle diaphorogona Foreman, 1973b, p. 258, pl. 2, figs 2-5; Foreman, 1975, p. 607, pl. 2F, figs. 1-5, pl. 3, figs. 1, 2. Acaeniotyle tribulosa Foreman, 1973b, p. 258, pl. 2, fig. 8 .

Acaeniotyle sp. aff. A. diaphorogona Foreman, 1973b, pl. 2, figs. 6, 7, pl. 16, fig. 16 .

Acaeniotyle umbilicata (Rüst, 1898)

(Pl. 6, Fig. 11; Pl. 15, Figs. 3a, b)

Xiphosphaera umbilicata Rüst, 1898, p. 7, pl. 1, fig. 9; Dumitrică, 1972, p. 832, pl. 1, fig. 1; Renz, 1974, p. 799, pl. 2, figs. 9-12, pl. 9, fig. 21.

Xiphosphaera tuberosa Tan Sin Hok, 1927, p. 35, pl. 5, fig. 8.

Spumellariinid Pessagno, 1969, p. 610, pl. 4, fig. N.

Acaeniotyle umbilicata (Rüst) in Foreman, 1973b, p. 258, pl. 1, figs. 13, 14,16 ; Foreman, 1975 , p. 607 , pl. 2 E, figs. $14-17$, pl. 3 , fig. 3 .

\section{Acanthocircus carinatus Foreman, 1973}

(P1. 16, Fig. 2)

Acanthocircus carinatus Foreman, 1973b, p. 260, pl. 5, fig. 1, 2; Foreman, 1975, p. 610, pl. 2C, fig. 8, pl. 4, fig. 12; (not Riedel and Sanfilippo, 1974, p. 774 , pl. 2, figs. 1-2).

Spongosaturnalis variabilis Squinabol in Moore, 1973, p. 824, pl. 6 , figs. 1,3 (not fig. 2).

\section{Acanthocircus dicranacanthos (Squinabol, 1914)}

(Pl. 7, Fig. 1; Pl. 16, Fig. 3)

Saturnalis dicranacanthos Squinabol, 1914, p. 289, fig. 1, pl. 22, figs. 4,6 (not figs. 5,7), pl. 23, fig. 8 .

Saturnalis novalensis Squinabol, 1914, p. 297, pl. 20, fig. 1, pl. 23, fig. 7.

Saturnulus sp. Fischli, 1916, p. 46, fig. 53 .

Spongosaturnalis dicranacanthos (Squinabol) in Pessagno, 1969, p. 610 , pl. 4, figs. A, B; Moore, 1973, p. 824, pl. 3, figs. 1, 3.

Acanthocircus dizonius (Rüst) in Foreman, 1973b, p. 260, pl. 4, figs. 4,5; Riedel and Sanfilippo, 1974, pl. 2, figs. 4, 5 (not fig. 3).

Acanthocircus dicranacanthos (Squinabol) in Foreman, 1975, p. 610, pl. 2D, figs. 5, 6; Pessagno, 1977a, p. 73, pl. 3, fig. 5; Pessagno, 1977b, p. 31, pl. 2, fig. 6.
Acanthocircus trizonalis (Rüst, 1898)

(PI. 16, Fig. 1)

Saturnulus trizonalis Rüst, 1898, p. 9, pl. 2, fig. 4; Fischli, 1916, p. 46, fig. 52.

Saturnalis amissus Squinabol, 1914, p. 296, pl. 23, figs. 2-5.

Saturnalis? aff. amissus Squinabol in Zhamoida, 1969, p. 19, fig. 9, p. 24.

Spongosaturnalis amissus (Squinabol) in Moore, 1973, p. 824, pl. 3, fig. 2.

Acanthocircus trizonalis (Rüst) (?) in Foreman, 1973b, p. 261, pl. 4, figs. 6-8.

Acanthocircus dizonius (Rüst) in Riedel and Sanfilippo, 1974, pl. 2, fig. 3 (not figs. 4,5 ).

\section{Acanthocircus sp.}

(P1. 7, Fig. 7)

Remarks. Elliptical saturnalin ring with two blades on the outer margin and with a single flat spine at each end.

\section{Alievium antiguum Pessagno, 1972}

(PI. 7, Fig. 10; Pl. 8, Fig. 2)

Alievium antiguum Pessagno, 1972, p. 298, pl. 24, figs. 1-4; Pesagno, 1977b, p. 29, pl. 3, figs. 14, 17, 21, 22.

Remarks. Some specimens have longer primary spines than those described by Pessagno (1972).

\section{Alievium helenae Schaaf, n. sp.}

(Pl. 7, Fig. 9; Pl. 10, Figs. 2a, b)

Alievium sp. Foreman, 1973b, p. 262, pl. 9, figs. 1, 2; Foreman, 1975, p. 613 , pl. 2D, figs. 7,8 , pl. 5 , fig. 14 .

Alievium sp. A Pessagno, 1977b, p. 29, pl. 3, figs. 10, 18.

Description. Test subspherical with three stout spines which are three-bladed, the three blades becoming much wider at their edges so as to be trefoil in section. Meshwork consisting of large equilateral triangular pore frames. Small secondary spines, circular in transverse section, arise from the nodes of the triangular meshwork.

Measurements. (Based on 11 specimens from Site 463, between core $89-1$ and 90 CC.) Minimum diameter of skeleton $145 \mu \mathrm{m}$; its maximum diameter (without spines) $195 \mu \mathrm{m}$, minimum length of spines $105 \mu \mathrm{m}$, their maximum length $210 \mu \mathrm{m}$.

Type locality. Mid-Pacific Mountains, Site 463 (see Site 463 chapter, this volume), Cores 89 and 90 .

Type specimens. Holotype: 62-463-90, CC, slide no. 11; England finder no. L 38/3. paratypes: $62-463-89-1,105-106 \mathrm{~cm}$, SEM negative no. $781064 ; 62-463-90, C C$, SEM negative no. 78980 .

Remarks. This species differs from $A$. superbum by having a more globular test with fewer triangular pores covering its surface, by being circular in outline and by having three bladed spines trefoil in section. This species is named in memory of Helen P. Foreman, in honor of her contributions to the study of Mesozoic Radiolaria.

\section{Amphibrachium? hastatum Renz, 1974}

(PI. 10, Fig. 4)

Amphibrachium (?) hastatum Renz, 1974, p. 788, pl. 1, figs. 1-6, pl. 9, fig. 1 .

Amphipyndax mediocris (Tan Sin Hok, 1927)

(Pl. 3, Fig. 11; Pl. 22, Figs. 7a, b)

Dictyomitra mediocris Tan Sin Hok, 1927, p. 55, pl. 10, fig. 82

Stichocapsa stocki Campbell and Clark, 1944, p. 44, pl. 8, figs. 31-33.

Stichocapsa megalocephalia Campbell and Clark, 1944, p. 44, pl. 8, figs, 26, 34.

Dictyomitra uralica Kozlova and Gorbovets, 1966, p. 116, pl. 6, figs. 6-7.

Amphipyndax stocki (Campbell and Clark) in Foreman, 1968, p. 78, pl. 81 , figs. $12 \mathrm{a}-\mathrm{c}$; Foreman, $1973 \mathrm{a}$, p. 430 , pl. 13, fig. 5; Petrushevskaya and Kozlova, 1972, p. 545, pl. 8, figs. 16, 17; Moore, 1973, p. 827, pl. 11, fig. 6; Riedel and Sanfilippo, 1974, p. 775, pl. 11, figs. 1-3, pl. 15, fig. 11; Pessagno, 1975, p. 1016, pl. 3, figs. 4-8.

Amphipyndax mediocris (Tan Sin Hok) in Renz, 1974, p. 788, pl. 5, figs. $7-9$, pl. 12, fig. 3 . 
Archaeodictyomitra apiara (Rüst, 1885)

(Pl. 18, Figs. 2a, b)

Lithocampe apiarum Rüst, 1885 , p. 314 , pl. 39, fig. 8 .

Dictyomitra sp. ind. C. Hinde, 1900, p. 39, pl. 3, fig. 13.

Lithomitra excellens Tan Sin Hok, 1927, p. 56, pl. 11, fig. 85; Moore, 1973, pl. 827, pl. 4, figs. 3, 4.

Lithostrobus dignus Tan Sin Hok, 1927, p. 54, pl. 11, fig. 79.

Dictyomitra apiarum (Rüst) in Foreman, 1975, p. 613, pl. 2G, figs. 7-8.

Archaeodictyomitra apiara (Rüst) in Pessagno, 1977b, p. 41, pl. 6, figs. 6,14 .

Archaeodictyomitra brouweri var. $\alpha$ (Tan Sin Hok, 1927)

(Pl. 19, Figs. 3a, b)

Eucyrtidium brouweri var. $\alpha$ Tan Sin Hok, 1927, p. 58, pl. 11, fig. 93. Dictyomitra sp. cf. Eucyrtidium brouweri Tan Sin Hok in Foreman, 1971, p. 1682, pl. 5, fig. 15; Moore, 1973, p. 830, pl. 14, figs. 7-9. Dictyomitra brouweri var. $\alpha$ (Tan Sin Hok) in Renz, 1974, p. 790, pl. 8 , figs. 14-16, pl. 11, fig. 26.

\section{Archaeodictyomitra lacrimula (Foreman, 1973)}

(Pl. 22, Figs. 3a, b)

Dictyomitra (?) lacrimula Foreman, 1973b, p. 263, pl. 10, fig. 11; Foreman, 1975, p. 614 , pl. 2G, figs. 5, 6, pl. 6, fig. 1 .

Cornutana conica Aliev in Moore, 1973, p. 830, pl. 14, figs. 1, 2, (not Aliev, 1965, pl. 6, fig. 1).

Remarks. I have not followed Pesagno (1977b, p. 45), who regarded Dictyomitra (?) lacrimula Foreman as a junior synonym of Cornutana conica Aliev and placed these two species in the genus Thanarla. Archaeodictyomitra lacrimula (Foreman) is less broad than Cornutana conica Aliev, and does not possess bladelike terminal feet as in the definition of Pessagno's genus Thanarla. As used here, the species has no intersegmental constrictions externally, is amphiconical, and has a very small aperture which is sometimes tubular.

\section{Archaeodictyomitra nuda Schaaf, n. sp.}

$$
\text { (P1. 3, Fig. 6) }
$$

Description. Smooth, conical form with approximately eight widely separated transverse rows of small pores at the internal septa. The scanning electron microscope reveals low, rather irregular ribs on the surface.

Measurements. (Based on 8 specimens from Site 463, between $89-1$ and $90, C$ C.) Length of the first six segments 155 to $190 \mu \mathrm{m}$, number of pores per half circumference of the fourth segment 12 to 14 .

Type locality. Mid-Pacific Mountains, Site 463 (see Site 463 report, this volume), Cores 89 and 90 .

Type specimens. Holotype: $62-463-90, C C$, slide no. 7; England finder no. H 27/2. Paratypes: $62-463-90, C C$, SEM negative no. 781098; 62-463-89-1, 94-95 cm, SEM negative no. 79066.

Remarks. This species differs from all others of the genus by the indistinct costae. Nuda (Latin, adj.), "naked."

\section{Archaeodictyomitra pseudoscalaris (Tan Sin Hok, 1927)}

$$
\text { (Pl. 4, Fig. 5; Pl. 21, Figs. 13a, b) }
$$

Stichomitra pseudoscalaris Tan Sin Hok, 1927, p. 56, pl. 11, fig. 84; (not Renz, 1974, pl. 8, figs. 5, 6, pl. 11, fig. 34).

Remarks. In some specimens, pores are less regularly arranged than in the original figure, and intersegmental structures are sometimes expressed in the external contour.

\section{Archaeodictyomitra puga Schaaf, n. sp.}

(Pl. 3, Fig. 7; Pl. 21, Figs. 11a, b)

Dictyomitra (?) sp. Dumitrică, 1973, pl. 4, fig. 7.

Description. Conical skeleton of usually 8 to 11 segments, and more or less pronouncedly undulating outline. At the wide levels of the shell are intersegmental septa, each of them associated with two transverse rows of pores. The pores of each row are longitudinally aligned, and alternate with costae which are continuous from segment to segment. Some specimens with no evident costae tend to have somewhat wider skeletons.

Measurements. (Based on 6 specimens from Site 463, between $89-1,94-95 \mathrm{~cm}$ and $90, \mathrm{CC}$.) Length of the eight first segments 180 to
$210 \mu \mathrm{m}$, number of pores per half circumference of the fifth segment 10 to 12 .

Type locality. Mid-Pacific Mountains, Site 463 (see Site 463 report, this volume), Cores 89 and 90 .

Type specimens. holotype: $62-463-89-1,15-16 \mathrm{~cm}$, slide no. 8; England finder no. D 40/1. Paratypes: $62-463-89-1,94-95 \mathrm{~cm}$, SEM negative nos. 78942 and 781154 .

Remarks. This species is distinguished from all others of the genus by the two rows of pores at junctions between segments. Puga, name formed by an arbitrary combination of letters.

\section{Archaeodictyomitra vulgaris Pessagno, 1977}

(Pl. 4, Fig. 2)

Dictyomitra sp. Foreman, 1973b, pl. 10, fig. 8.

Archaeodictyomitra vulgaris Pessagno, 1977b, p. 44, pl. 6, fig. 15.

\section{Archaeospongoprunum cortinaensis Pessagno, 1973}

$$
\text { (Pl. 7, Fig. 11) }
$$

Archaeospongoprunum cortinaensis Pessagno, 1973, p. 60, pl. 9, figs. 4-6; Pessagno, 1976, p. 33, pl. 1, fig. 3.

Remarks. The preservation of our material does not permit recognition of the several species illustrated by Pessagno (1973). We group under $A$. cortinaensis the species with one polar spine in clockwise arrangement, and under $A$. tehamaensis the species which lack a spiral arrangement.

\section{Archaeospongoprunum tehamaensis Pessagno, 1973}

$$
\text { (Pl. 7, Figs. 3,5; Pl. 10, Figs. 7a, b) }
$$

Archaeospongoprunum tehamaensis Pessagno, 1973, p. 65 , pl. 9 , figs. 2-3; Pessagno, 1976, p. 33, pl. 1, fig. 1 .

Archaeospongoprunum sp. A. Pessagno, 1977b, p. 30, pl. 2 , fig. 2.

\section{Archicapsa similis Parona, 1890}

(Pl. 22, Figs. 4,5; Pl. 23, Fig. 7)

Archicapsa similis Parona, 1890, p. 163, pl. 5, fig. 4; Hinde, 1900, p. 28 , pl. 3 , fig. 22 ; Moore, 1973 , p. 825 , pl. 16 , figs. $3-4$.

\section{Artostrobium tina Foreman, 1971}

(Pl. 24, Figs. 6a, b)

Artostrobium tina Foreman, 1971, p. 1678, pl. 4, fig. 3; Moore, 1973, p. 826 , pl. 8 , fig. 6 ; Foreman, 1975 , p. 613 , pl. 1F, figs. 3-5, pl. 6 , fig. 5 .

\section{Conosphaera tuberosa Tan Sin Hok, 1927}

(Pl. 12, Figs. 5a, b)

Conosphaera tuberosa Tan Sin Hok, 1927, p. 36, pl. 6, fig. 10.

?Conosphaera tuberosa Tan Sin Hok in Renz, 1974, p. 789, pl. 2, figs. 6-8, pl. 17, fig. 17 .

\section{Crolanium pythiae Schaaf, n. sp.}

(Pl. 20, Figs. 5a-c)

Dictyomitra (?) sp. Foreman, 1975, p. 615, pl. 2H, fig. 4.

Description. The shell is conical and consists of 10 to 12 uniform segments which increase gradually in length distally. Characteristic of this form are the three tubular feet which permit recognition even in poorly preserved material. Externally there is little or no segmental division, except for sometimes very slight constrictions. Pores are small, rounded, arranged randomly or in transverse rows.

Measurements. (Based on 9 specimens from Site 463, Cores 75 to 79.) Length 250 to $420 \mu \mathrm{m}$; greatest width without the tubular feet 130 to $180 \mu \mathrm{m}$.

Type locality. Mid-Pacific Mountains, Site 463 (see Site 463 report, this volume), Cores 75 to 84 .

Type specimens. Holotype: $62-463-75-1,21-22 \mathrm{~cm}$, slide no. 35 ; England finder no. D 36/1. Paratype: $62-463-84-1,5-6 \mathrm{~cm}$, slide no. 8; England finder no. C 49/4.

Remarks. Crolanium pythiae differs from all others of the genus in having three tubular projections instead of solid spines. This species, which is known only from DSDP Leg 20, 196-4-1, and DSDP Leg 32, $307-7-1,75-77 \mathrm{~cm}$, occurs through more than 130 meters. The shape of this radiolarian is reminiscent of the seat of the Pythia, a principal 
personality in Greek mythology, Apollo's priestess in the panhellenic sanctuary of Delphi, and is therefore named pythiae.

\section{Cromyodruppa concentrica Lipman, 1960}

(Pl. 13, Figs. 8a, b)

Cromyodruppa concentrica Lipman, 1960, p. 124, pl. 26, figs. 11-14.

Crucella sp. cf. C. cachensis Pessagno, 1971

$$
\text { (Pl. 8, Fig. 3) }
$$

Crucella cachensis Pessagno, 1971, p. 53, pl. 9, figs. 1-3.

Remarks. This morphotype may be an ancestor of $C$. cachensis, and we take under this species all the forms with a central area markedly elevated.

\section{Cryptamphorella challengeri Schaaf, $\mathbf{n}$. sp.} (PI. 9, Figs. 6a, b)

Description. Shell inflated-pyriform, with cephalis and upper part of thorax forming a conical section, and abdomen sometimes irregular in outline. Cephalis relatively large, spherical. Thorax hemispherical, thin-walled, sometimes with small pores, and widely open, to a varying extent sunken into abdominal cavity. Abdominal wall moderate to thick with pores quincuncially arranged, varying in number and size (12-15 on half the equator).

Measurements. (Based on 21 specimens from Site 463, between $85-1,142-143 \mathrm{~cm}$ and $88-1,52-53 \mathrm{~cm}$.) Diameter of shell 90 to $105 \mu \mathrm{m}$; diameter of sutural pore 6 to $8 \mu \mathrm{m}$.

Type locality. Mid-Pacific Mountains, Site 463 (see Site 463 report, this volume), Cores 85 to 88 .

Type specimens. Holotype: $62-463-88-1,52-53 \mathrm{~cm}$, slide no. 5; England finder no. X 34/4. Paratype: $62-463-88-1,52-53 \mathrm{~cm}$, slide no. 3; England finder no. $\mathrm{N} 48 / 2$.

Remarks. Differs from Cryptamphorella dumitricai Schaaf, n. sp. as indicated under that species. This species is named for the $\mathrm{D} / \mathrm{V}$ Glomar Challenger, whose tenth anniversary was celebrated during Leg 62 .

\section{Cryptamphorella conara (Foreman, 1968)}

(Pl. 1, Figs. 6a, b; Pl. 9, Figs. 15a, b)

Hemicryptocapsa conara Foreman, 1968, p. 35, pl. 4, figs. 11a-b. Cryptamphorella conara (Foreman) in Dumitrică, 1970, p. 80, pl. 11, figs. 66a-c; Dumitrică, 1972 , p. 842 , pl. 1, figs. 2-5.

\section{Cryptamphorella dumitricai Schaaf, n. sp.}

(Pl. 1, Figs. 5a-c; Pl. 9, Figs. 5a,b, 13a, b)

Description. Shell spherical, with a small, free, poreless cephalis, without apical horn. Collar structure indistinct. Cephalo-thorax more or less depressed into abdominal cavity. Abdomen spherical, with wall moderate to thin, without aperture, but with a small, circular sutural pore always prominent. The top of the abdomen appears to be flat in optical section, but not as observed by the scanning electron microscope. Pores regular in size, with pentagonal or hexagonal frames.

Measurements. (Based on 9 specimens from Site 463, between $89-1,94-95 \mathrm{~cm}$, and 90, CC.) Diameter of shell 85 to $155 \mu \mathrm{m}$; diameter of sutural pore 4 to $8 \mu \mathrm{m}$.

Type locality. Mid-Pacific Mountains, Site 463 (see Site 463 report, this volume), Cores 89 and 90 .

Type specimens. Holotype: 62-463-90,CC, slide no. 2; England finder no. X 43/2. Paratype: $62-463-89-1,94-95 \mathrm{~cm}$, SEM negative nos. 781126 to 781128 .

Remarks. This species differs from Cryptamophorella challengeri, n. sp. by the prominent sutural pore and the apparently flat top of the abdomen. This species is named for Dr. Paulian Dumitrică, in honor of his contributions to the study of cryptocephalic and cryptothoracic Nassellaria.

\section{Cyclastrum infundibuliforme Rüst, 1898}

(Pl. 14, Fig. 8)

Cyclastrum infundibuliforme Rüst, 1898, p. 28, pl. 9, fig. 5 .

\section{Cyrtocalpis operosa Tan Sin Hok, 1927}

(Pl. 27, Figs. 8a, b)

Cyrtocalpis operosa Tan Sin Hok, 1927, p. 40, pl. 7, fig. 27; Riedel and Sanfilippo, 1974, p. 778, pl. 4, figs. 1-3, pl. 14, fig. 10; Renz, 1974 , p. 790 , pl. 4 , figs. 15 , 16 , pl. 12 , fig. 8 .

\section{[?]Cyrtocapsa asseni var. $\alpha$ Tan Sin Hok, 1927}

(Pl. 6, Fig. 9)

Cyrtocapsa asseni var. $\alpha$ Tan Sin Hok, 1927, p. 67, pl. 14, fig. 119.

Cyrtocapsa grutterinki Tan Sin Hok, 1927

(Pl. 6, Figs. 6a, b)

Cyrtocapsa grutterinki Tan Sin Hok, 1927, p. 64, pl. 13, fig. 110.

\section{Cyrtocapsa houwi Tan Sin Hok, 1927}

(Pl. 22, Fig. 2)

Cyrtocapsa houwi Tan Sin Hok, 1927, p. 6, pl. 1, fig. 11.

Diacanthocapsa boersmae Schaaf, n. sp.

(Pl. 9, Figs. 2a, b, 12a, b)

Description. Shell variably ovoid to amphiconical. Cephalis small, spherical. Thorax inflated-hemispherical, with thin wall and very few pores, partly sunken into abdomen, and with cavity not much smaller than abdominal cavity. Sutural pore in lumbar position has not been observed. Abdomen thick-walled, with widely separated small pores.

Measurements. (Based on 15 specimens from Site 463, between $88-1,52-53 \mathrm{~cm}$ and $92-1,0-1 \mathrm{~cm}$.) Diameter of shell 60 to $70 \mu \mathrm{m}$; diameter of cephalis 15 to $20 \mu \mathrm{m}$.

Type locality. Mid-Pacific Mountains, Site 463 (see Site 463 report, this volume), Cores 88 to 92 .

Type specimens. Holotype: $62-463-92-1,0-1 \mathrm{~cm}$, slide no. 2; England finder no. N 44/4. Paratypes: $62-463-85-1,142-143 \mathrm{~cm}$, slide no. 4; England finder no. K 45/2; 62-463-88-1, 52-53 cm, slide no. 5; England finder no. X 35/4.

Remarks. This species is smaller than described members of Diacanthocapsa, and has fewer pores on the thorax. This species is named for Anne Boersma, in honor of her contributions to the study of Cretaceous and Paleocene foraminifers.

Dibolachras tytthopora Foreman, 1973

(PI. 5, Figs. 3a, b; Pl. 26, Figs. 1a, b, 4)

Dibolachras tytthopora Foreman, 1973b, p. 265, pl. 11, fig. 4, pl. 16, fig. 15; Foreman, 1975, p. 617, pl. 2L, figs. 2, 3, pl. 6, fig. 16.

Remarks. Although the external contour of the fourth segment is rounded, its internal surface presents an angular contour, as shown by the internal mold in Plate 26, Figure 4.

\section{Dicroa sp. A Foreman, 1975 \\ (Pl. 16, Fig. 8)}

Dicroa sp. A Foreman, 1975, p. 609, pl. 2E, figs. 9-11, pl. 3, fig. 11.

\section{Dictyophimus gracilis Tan Sin Hok, 1927}

(Pl. 5, Fig. 10)

Dictyophimus gracilis Tan Sin Hok, 1927, p. 42, pl. 7, fig. 33; Renz, 1974 , p. 791 , pl. 5 , figs. $14-16$, pl. 11, fig. 11.

Napora lospensis Pessagno, 1977a, p. 96, pl. 12, figs. 9-10.

\section{Eucyrtidium thiensis Tan Sin Hok, 1927}

(Pl. 27, Figs. 6a, b)

Eucyrtidium thiensis Tan Sin Hok, 1927, p. 60, pl. 11, fig. 95.

Not Eucyrtidium sp. cf. E. thiensis Tan Sin Hok in Moore, 1973, p. 829 , pl. 7, fig. 6; Renz, 1974, p. 792, pl. 11, fig. 23.

\section{Eucyrtis sp. cf. E. bulbosa Renz, 1974}

$$
\text { (Pl. 26, Fig. 2) }
$$

Eucyrtis bulbosus Renz, 1974, p. 792, pl. 7, figs. 26-29, pl. 12, figs. $15 \mathrm{a}-\mathrm{b}$; not Foreman, 1975, pl. 2K, fig. 35. 
Remarks. Forms encountered here conform well with Renz's description, but only some of the forms have the distal tube preserved.

Eucyrtis columbaria Renz, 1974

(Pl. 5, Figs. 1a, b; Pl. 27, Figs. 2a, b, 3a, b)

Eucyrtis columbarius Renz, 1974, p. 792, pl. 7, figs. 14-20, pl. 12, figs. 13a-c.

Eucyrtis columbaria Renz in Foreman, 1975, pl. 2I, fig. 19.

Remarks. In well-preserved specimens, there are three horns, the long apical horn arising from one side of the cephalis, and two short subsidiary horns apparently from the thoracic wall near the collar structure.

Eucyrtis elido Schaaf, n. sp.

(Pl. 5, Fig. 6; Pl. 25, Figs. 3a, b)

Eucyrtis hanni (Tan Sin Hok) in Riedel and Sanfilippo, 1974, p. 779, pl. 5, figs. 9, 10; Renz, 1974, p. 792, pl. 7, fig. 24 .

Description. Shell sub-spindle-shaped. Cephalis small and poreless, bearing a stout horn. The conical proximal segments increase gradually in width. The fifth and sixth segments are the widest. The two or three following (last) segments decrease in width, but not in length. Pores are small, rounded, staggered on the three first segments, larger and arranged randomly or in vague transverse rows on the other segments. The surface is spiny, with long, upwarddirected spines proximally, and shorter, downward-directed spines distally.

Measurements. (Based on 13 specimens from Site 463, 90,CC.) Diameter of cephalis 8 to $15 \mu \mathrm{m}$; greatest width 65 to $75 \mu \mathrm{m}$, length of the five first segments 130 to $160 \mu \mathrm{m}$.

Type locality. Mid-Pacific Mountains, Site 463 (see Site 463 report, this volume), Core 90.

Type specimens. Holotype: $62-463-90, \mathrm{CC}$, slide no. 10; England finder no. $\mathrm{H} \mathrm{38/2}$. Paratype: 62-463-90,CC, slide no. 5; England finder no. L 40/3.

Remarks. This species is distinguished from E. micropora and $E$. tenuis by the size of its pores and the shape of the three last segments. It differs from $E$. hanni in not constricting so abruptly and in having a thinner wall. Elido, name formed by an arbitrary combination of letters.

\section{Eucyrtis molengraaffi (Tan Sin Hok, 1927)}

(Pl. 27, Fig. 5a, b)

Syringium molengraaffi Tan Sin Hok, 1927, p. 63, pl. 13, fig. 105. Eucyrtis molengraaffi (Tan Sin Hok) in Renz, 1974, p. 792, pl. 7, figs. $1-4$, pl. 11 , fig. 32

\section{Eucyrtis tenuis (Rüst, 1885) s.1. (Plate 25, Fig. 8)}

Stichocapsa tenuis Rüst, 1885 , p. 318 , pl. 47 , figs. 13-14; Riedel and Sanfilippo, 1974, pl. 9, figs. 13, 14

Eucyrtis tenuis (Rüst) in Foreman, 1975, p. 615, pl. 2I, figs. 7-9. Archicapsa micropora Squinabol, 1903, p. 129, pl. 9, fig. 14

Eusyringium sp. A Zhamoida in Zhamoida et al., 1968, pl. 1, fig. 8; Zhamoida, 1969, pl. 19, fig. 8; Zhamoida, 1972, p. 121, pl. 17, fig. 3.

Eucyrtis zhamoidai Foreman, 1973b, pl. 10, figs. 9, 10, pl. 16, fig. 1. [?] Eucyrtis zhamoidai Foreman, 1973b, pl. 16, fig. 2.

Eucrytis micropora (Squinabol) in Foreman, 1975, p. 615, pl. 2I, figs. $2-5$.

Remarks. Because the Leg 62 material is not sufficiently well preserved to permit the distinction of Eucyrtis micropora from $E$. tenuis, both forms are here recorded under the latter name in its broad sense.

\section{?Excentropylomma cenomana Dumitrică, 1970}

(Pl. 24, Figs. 8a, b)

Excentropylomma cenomana Dumitrică, 1970, p. 77, pl. 15, figs. 96a, b, $97,98 \mathrm{a}, \mathrm{b}$.

\section{Gongylothorax baumgartneri Schaaf, n. sp.}

$$
\text { (Pl. 9, Figs. 1a, b) }
$$

Description. Subspherical form with cephalis protruding only very slightly. Cephalis small, thick-walled, within wall of thorax. Thorax tending to be ovoid, pointed basally, with a small, indistinct mouth significantly larger than a pore. Thoracic wall thick, with smooth surface and rather large pores separated by bars approximately as wide as the pore diameters.

Measurements. (Based on 6 specimens from Site 463, between 90,CC and 92-1, 0-1 cm.) Diameter of shell 190 to $240 \mu \mathrm{m}$; diameter of pores 6 to $10 \mu \mathrm{m}$.

Type locality. Mid-Pacific Mountains, Site 463 (see Site 463 report, this volume), Core 90

Type specimens. Holotype: $62-463-90, \mathrm{CC}$, slide no. 2; England finder no. T 45/3. Paratype: $62-463-90, C C$, slide no. 7; England finder no. X 24/2

Remarks. This species is larger than the co-occurring threesegmented form Holocryptocapsa (?) sp. cf. H. cryptodon (Dumitrică, 1972) with which it occurs, and it has more pores and two segments. This species is named for Peter Baumgartner (Geological Institute, Basel) in honor of his contribution to the study of Late Jurassic-Early Cretaceous Radiolaria.

\section{Gongylothorax verbeeki (Tan Sin Hok, 1927)}

(Pl. 1, Figs. 1a, b; Pl. 9, Figs. 9a, b)

Dicolocapsa verbeeki Tan Sin Hok, 1927, p. 44, pl. 8, figs. 40, 41; (not Foreman, 1968, p. 20, figs. $8 \mathrm{a}-\mathrm{c}$; Dumitrică, 1970, p. 57 , pl 1 , figs. $6 a$, b, pl. 2, figs. 7-10).

Gongylothorax favosus Dumitrică, 1970, p. 56, pl. 1, figs. 1a-c, 2.

Remarks. The form described by Dumitrică as $G$. favosus corresponds well with specimens of Tan Sin Hok's Dicolocapsa verbeeki in his sample 150 from Rotti. The names are therefore synonymized, and the form assigned to $G$. verbeeki by Dumitrică and by Foreman is thus left to be named by the next worker studying it. Specimens from Leg 62 vary in the degree of development of the hexagonal pore frames, and in that some have thorns at the junctions of the pore frames.

\section{Hagiastrum? subacutum Rüst, 1885}

(Pl. 11, Fig. 4)

Hagiastrum subacutum Rüst, 1885 , p. 229 , pl. 34 , fig. 1.

\section{Hagiastrum? euganeum (Squinabol, 1903)}

(Pl. 11, Figs. 1a, b)

Stauralastrum euganeum Squinabol, 1903, p. 123, pl. 9, fig. 19.

\section{Hemicryptocapsa? ef. H. cryptodon (Dumitrică, 1970)}

(Pl. 9, Figs. 7a, b)

?Hemicryptocapsa cryptodon Dumitrică, 1970 , p. 73 , pl. 14, figs. 90 9la-c.

Holocryptocapsa? sp. cf. H. cryptodon (Dumitrică) in Dumitrică, 1972 , p. 841 , pl. 2 , figs. $3-6$.

Hemicryptocapsa sp. cf. $H$. prepolyhedra Dumitrică, 1970 (Pl. 1, Fig. 4; Pl. 9, Figs. 8a, b)

[?] Hemicryptocapsa prepolyhedra Dumitrică, 1970, p. 71 , pl. 13, figs. 80a-c, 81-83a-b, 84, pl. 20, fig. 131.

Remarks. This species is easily distinguishable from Williriedellum peterschmitti, n. sp. by the presence of a sutural pore.

\section{Hemicryptocapsa pseudopilula Tan Sin Hok, 1927

$$
\text { (P1. 2, Figs. 5a, b) }
$$

Hemicryptocapsa pseudopilula Tan Sin Hok, 1927, p. 51, pl. 9, fig. 69.

\section{Hemicryptocapsa vincentae Schaaf, n. sp.}

(Pl. 9, Figs. 10a, b)

Description. Small, inflated-pyriform shell with rough surface. Cephalis spherical, without pores. No distinct collar stricture. Thorax hemispherical, wide open, partly sunken into abdominal cavity, with distinct apertural spines. Abdominal wall appears rather thick as a result of prominent polygonal frames around the widely spaced, circular pores.

Measurements. (Based on 5 specimens from Site 463, between $86, \mathrm{CC}$ and $90, \mathrm{CC}$ ). Diameter of shell 85 to $100 \mu \mathrm{m}$; diameter of cephalis 15 to $25 \mu \mathrm{m}$. 
Type locality. Mid-Pacific Mountains, Site 463 (see Site 463 report, this volume), Cores 86 to 90 .

Type specimens. Holotype: $62-463-86, \mathrm{CC}$, slide no. 3; England finder no. R 56/3. Paratype: 62-463-90,CC, slide no. 7; England finder no. $\mathrm{K} 22 / 2$.

Remarks. This species is smaller than $H$. pseudopilula Tan Sin Hok, and has fewer, more widely separated pores. This species is named for Edith Vincent, in honor of her contributions to the study of Cenozoic foraminifers.

\section{Hexalonche? sp. group \\ (Pl. 12, Figs. 2, 4a, b)}

Description. These species have been found only as internal moulds, except that a medullary shell remains in some specimens. The medullarly shell has closely spaced pores, approximately 5 to 7 on half a circumference, and is joined to the cortical shell by 6 to 8 radial beams, which can sometimes be seen to be three-bladed. When they number six, they are arranged regularly in cubic axes. Some specimens show traces of the pores of the cortical shell, about 25 to 30 on half a circumference. Very likely, the beams extended as external spines on the original skeletons.

Measurements. Diameter of medullary shell 35 to $55 \mu \mathrm{m}$; diameter of cortical shell 140 to $190 \mu \mathrm{m}$.

Remarks. Some specimens seem to show two phases of infilling. It is difficult to explain why these forms are found only as internal moulds. They could be reworked from older sediments, but then they would be accompanied by other, older species, which is not found to be the case. The only other forms in this assemblage filled with redeposited silica are some spongodiscids and occasional stichocyrtids.

\section{Hexastylurus magnificus (Squinabol, 1904)}

(Pl. 7, Fig. 2; Pl. 14, Figs. 1a, b)

Staurosphaera magnifica Squinabol, 1904, p. 191, pl. 3, fig. 1.

Remarks. The Leg 62 specimens certainly have six spines, and Squinabol's may also have had six. This species is therefore assigned to Hexastylurus Haeckel, which has an appropriate definition and also a type species (Hexastylurus dictyotus Haeckel, 1887), which is at least superficially similar.

\section{Hexinastrum cretaceum Lipman, 1960}

$$
\text { (Pl. 14, Fig. 6) }
$$

Hexinastrum cretaceum Lipman, 1960, p. 133, pl. 30, fig. 8. Pentinastrum subbotinae Lipman, 1960, p. 132, figs. 8a-b, pl. 30 , figs. 6-7.

\section{Higumastra sp.}

(Pl. 6, Fig. 12; Pl. 10, Figs. 5a, b)

Remarks. We follow in this work Baumgartner's (in press) genus conception.

\section{Histiastrum aster Lipman, 1952}

(Pl. 8, Fig. 1; Pl. 11, Fig. 5)

Histiastrum aster Lipman, 1952, p. 35, pl. 11, figs. 6-7; Lipman, 1962, p. 300, pl. 2, fig. 5; Kozlova and Gorbovets, 1966, p. 84, pl. 3, fig . 9.

Holocryptocanium barbui Dumitricà, 1970

(P1. 2, Figs. 1a, b; Pl. 10, Figs. 6a, b)

Holocryptocanium barbui Dumitrică, 1970, p. 76, pl. 17, figs. 105-108a, b, pl. 21, fig. 136.

\section{Holocryptocapsa hindei Tan Sin Hok, 1927}

(Pl. 9, Figs. 4a, b, 14)

Holocryptocapsa hindei Tan Sin Hok, 1927, p. 53, pl. 10, fig. 75 ; Dumitrică, 1970 , p. $74, \mathrm{pl} .15$, figs. $100 \mathrm{a}-\mathrm{d}$.

Remarks. All specimens are markedly flattened basally, and some also apically. Thus, this species, is easily distinguished from other cryptocephalic forms.
Homoeoparonaella tricuspidata (Rüst, 1898)

(Pl. 14, Fig. 5)

Chitonastrum tricusipdatum Rüst, 1898, p. 29, pl. 9, fig. 8 .

Hsuum sp. cf. H. obispoensis Pessagno, 1977

$$
\text { (Pl. 19, Fig. 4) }
$$

Hsuum obispoensis Pessagno, 1977a, p. 82, pl. 8, figs. 3-4; Pessagno, 1977b, p. 44 , pl. 6 , fig. 7.

\section{Lithocampe chenodes Renz, 1974 group}

(Pl. 5, Fig. 2; Pl. 25, Figs. 5a, b, 7)

Lithocampe chenodes Renz, 1974, p. 793, pl. 7, fig. 30, pl. 12, figs. 14a-d; Riedel and Sanfilippo, 1974, p. 779, pl. 6, figs. 5-7, pl. 13, fig. 1 .

Remarks. The shape and the size of this species are very variable. Some specimens are very similar in shape to Lithocampe mediodila tata Rust, 1885 (as described by Moore, 1973), but the number of rows of pores is larger. I propose and follow here a strict specific definition based on the number of rows of pores. Lithocampe chenodes has five or more rows of pores, whereas Lithocampe mediodilatata has only two rows of pores on each segment.

?Lithocampe pseudochrysalis var. $\alpha$ (Tan Sin Hok, 1927)

(Pl. 18, Figs. 4a, b)

Lithocampe pseudochrysalis var. $\alpha$ Tan Sin Hok, 1927, p. 64, pl. 13, fig. 108; Renz, 1974, p. 793, pl. 11, fig. 31.

\section{Lithomitra? pseudopinguis Tan Sin Hok, 1927}

(Pl. 19, Figs. 5a, b, 9a, b)

Lithomitra pseudopinguis Tan Sin Hok, 1927, p. 57, pl. 10, fig. 86; Renz, 1974, p. 794, pl. 7, figs. 5-7, pl. 11, fig. 30.

Remarks. As in Tan Sin Hok's material and illustrations, there are two morphotypes of $L$. pseudopinguis, one slender, with thin walls (Pl. 19, Figs. 5a, b), the other larger, with thick walls (PI. 19, Figs. $9 \mathrm{a}, \mathrm{b})$.

\section{Lithostrobus punctulatus Pessagno, 1963}

(Pl. 21, Figs. 5a, b)

Lithostrobus punctulatus Pessagno, 1963, p. 210, pl. 1, fig. 1, pl. 5, fig. 5 (not fig. 4); Foreman, 1978, p. 747, pl. 4, fig. 11

\section{Lophophaena sp. \\ (Pl. 21, Figs. 6a, b)}

Description. Three-lobed cephalis; very slight collar constriction; small pores, irregularly rounded and spaced.

\section{Mita magnifica Pessagno, 1977}

(Pl. 6, Fig. 10; Pl. 24, Figs. 3a, b, 13a, b)

Mita magnifica Pessagno, 1977b, p. 44, pl. 6, figs. 2, 5, 11, 13, 17, pl. 7 , fig. 24 , pl. 12 , fig. 11 .

\section{Neosciadocapsa sp. \\ (P1. 25, Figs. 4a, b)}

Remarks. Only a few specimens of this genus are present in the bottom of the cored sediments at Site 463 .

Obesacapsula somphedia (Foreman, 1973)

(Pl. 4, Figs. 6-9; Pl. 20, Figs. 1a, b, 2)

Dictyomitra somphedia Foreman, 1973b, p. 264, pl. 14, fig. 18; Foreman, 1975, p. 614, pl. 7, figs. 11-13.

Remarks. This species is very variable in shape and size.

Orbiculiforma chartonae Schaaf, n. sp.

(Pl. 8, Fig. 6; Pl. 13, Fig. 1)

Description: Circular, spongy, discoidal skeleton with pronouncedly differentiated center. The outer thickest zone of the disk is coarsely spongy; inwardly it is thinner and finer, with indistinct radial structure. At the center are two concentric lattice-spheres, the outer one appearing incomplete on the exposed surfaces. 
Measurements. (Based on 7 specimens from Hole 465A, between 28-1 and 29-1.) Diameter of entire skeleton 360 to $450 \mu \mathrm{m}$; diameter of outer spherical shell 50 to $80 \mu \mathrm{m}$.

Type locality. Hess Rise, Hole 465A (see Site 465 report, this volume), Cores 28 and 29.

Type specimens. Holotype: $62-465 \mathrm{~A}-29-1,43-44 \mathrm{~cm}$, slide no. 1; England finder no. W 47/3. Paratypes: 62-465A-29-1, 43-44 cm, SEM negative no. 79339; 62-466-34-2, 16-17 cm, slide no. 1; England finder no. O 52/1.

Remarks. Distinguished from other members of the genus by the different mesh sizes of the outer and inner spongy zones, and by the very distinct central spheres. It is not clear whether any of Pessagno's species of Orbiculiforma have central spheres, because most of his illustrations are scanning electron micrographs. This species is named after Dominique Charton, to thank her for her care in preparing the illustrations and plates.

\section{Paronaella? diamphidia Foreman, 1973}

$$
\text { (Pl. 13, Fig. 4) }
$$

Paronaella(?) diamphidia Foreman, 1973 b, p. 262 , pl. 8 , figs. $3-4$; Foreman, 1975 , p. 612 , pl. 5, figs. $4-5$.

\section{Paronaella sp. \\ (Pl. 8, Fig. 7)}

Remarks. The bad preservation of the end of the arms does not pernit a specific designation.

\section{Parvicingula sp. cf. P. altissima (Rüst, 1885)}

(Pl. 18, Figs. 1a, b)

Lithocampe altissima Rüst, 1885, p. 315 , pl. 15, fig. 2; Moore, 1973, pl. 3, fig. 7.

Parvicingula altissima (Rüst) in Pessagno, 1977a, p. 85, pl. 8, figs. 9-10.

Remarks. All the forms of this species recorded in Leg 62 are markedly smaller than in Rüst's description.

\section{Parvicingula boesii (Parona, 1890)}

(P1. 3, Figs. 13a, b; P1. 4, Fig. 13, Pl. 18, Figs. 6a, b)

Dictyomitra boesii Parona, 1890, p. 170, pl. 6, fig. 9; Riedel and Sanfilippo, 1974, p. 778 , pl. 4, figs. 5-6; Foreman, 1975, p. 613, pl. $2 \mathrm{H}$, figs. $10-11$, pl. 7 , fig. 9 .

Lithocampe ananassa Rüst, 1885, p. 315, pl. 40, fig. 3; Moore, 1973, p. 828 , pl. 4 , figs. 7-9.

Amphipyndax (?) sp. Foreman, 1973b, p. 263, pl. 9, figs. 3-4.

Parvicingula boesii (Parona) in Pessagno, 1977b, p. 48 , pl. 8, fig. 5 .

Mirifusus boesii (Parona) in Foreman, 1978, p. 746, pl. 2, fig. 6.

\section{Parvicingula hsui Pessagno, 1977}

$$
\text { (Pl. 3, Fig. 4) }
$$

Parvicingula hsui Pessagno, 1977a, p. 85, pl. 8, figs. 15-16, pl. 9, figs. 1-5.

\section{?Parvicingula malleola (Aliev, 1961) group}

$$
\text { (Pl. 21, Figs. 2, 12) }
$$

Dictyomitra malleola Aliev, 1961, p. 62, pl. 2, figs. 5, 6, 7; Aliev, 1965 , p. 48 , pl. 8 , figs. 4-6; ? Renz, 1974, p. 790 , pl. 8 , fig. 20 , pl. 11 , fig. 28 .

\section{Parvicingula? tekschaensis (Aliev, 1967) \\ (Pl. 3, Fig. 12; Pl. 20, Figs. 3a, b)}

Dictyomitra tekschaensis Aliev, 1967, p. 29, fig. K.

Dictyomitra spp. cf. D. tekschaensis Foreman, 1975, p. 615, pl. 1H, fig. 1 , pl. $2 \mathrm{H}$, fig. 1 .

\section{Patellula planoconvexa (Pessagno, 1963)}

$$
\text { (Pl. 8, Fig. 9) }
$$

Stylospongia planoconvexa Pessagno, 1963, p. 199, pl. 3, figs. 4-6, pl. 6 , fig. 1.

Patellula planoconvexa (Pessagno) in Petrushevskaya and $\mathrm{Koz}-$ lova, 1972, p. 527 , pl. 3, fig. 13 .
Pentasphaera longispina Squinabol, 1904

(Pl. 6, Fig. 14)

Pentasphaera longispina Squinabol, 1904, p. 193, pl. 5, fig. 1.

Podobursa triacantha (Fischli, 1916)

(PI. 5, Fig. 11; Pl. 25, Figs. 1a, b)

Theosyringium acanthophorum Rüst var. triacanthus Fischli, 1916, p. 47 , fig. 38 .

Theosyringium acanthophorum Rüst var. tetracanthus Fischli, 1916, p. 47 , fig. 39 .

Theosyringium acanthophorum Rüst var. polyacanthus Fischli, 1916, p. 47 , fig. 41 .

Podobursa triacantha (Fischli) in Foreman, 1973b, p. 266, pl. 13, figs. 1-3; Foreman, 1975, p. 617, pl. 2L, figs. 4-6; Pessagno, 1977b, p. 57 , pl. 11 , fig. 6 .

Podobursa sp. Riedel and Sanfilippo, 1974, pl. 13, fig. 7.

Podobursa tricola Foreman, 1973

(Pl. 6, Figs. 1a, b; P1. 25, Figs. 2a, b)

Podobursa tricola Foreman, 1973b, p. 267, pl. 13, fig. 9, pl. 16, fig. 12 ; Foreman, 1975 , p. 617 , pl. 2 L, figs. $7-8$.

\section{Pseudoaulophacus? excavatus (Rüst, 1898)}

$$
\text { (Pl. 12, Fig. 6) }
$$

Stylotrochus excavatus Rüst, 1898, p. 35, pl. 12, fig. 4.

Pseudoaulophacus? sulcatus (Rüst, 1898)

(Pl. 14, Figs. 3a, b)

Astrocyclia sulcata Rüst, 1898, p. 21, pl. 7, fig. 2.

Pseudodictyomitra blabla Schaaf, n. sp. (Pl. 21, Figs. 1a, b)

Description. Conical shell of 6 to 7 segments, the last one inverted truncate-conical. Intersegmental structures pronounced, less marked externally. Each segment has a single row of pores distally, and all except the last two segments have external ribs on their lower halves.

Measurements. (Based on 8 specimens from Site 463, between $84-1,5-6 \mathrm{~cm}$ and $86, C$ C.) Length of the six first segments 115 to 130 $\mu \mathrm{m}$; number of rows of pores per half circumference on the fifth segment 10 to 12 .

Type locality. Mid-Pacific Mountains, Site 463 (see Site 463 report, this volume), Core 86 .

Type specimens. Holotype: $62-463-86, \mathrm{CC}$, slide no. 4; England finder no. Y 31/4. Paratype: $62-463-86, C C$, slide no. 2; England finder no. B $7 / 1$.

Remarks. This species is distinguished by its small size, lack of ribs on the last two segments, and the tapered last segment. This form also occurs in Tan Sin Hok's (1927) samples 149 and 154, although he did not record it. Blabla, name formed by an arbitrary combination of letters.

\section{Pseudodictyomitra carpatica (Lozyniak, 1969)}

(Pl. 3, Figs. 1a-c, 2; Pl. 20, Figs. 4a, b)

Dictyomitra carpatica Lozyniak, 1969, p. 38, pl. 2, figs. 11-13.

Dictyomitra carpatica Lozyniak (?) in Foreman, 1973b, p. 263, pl. 10, figs. 1-3, pl. 16, fig. 5; Foreman, 1975, p. 614, pl. 2G, figs. 11-14, pl. 7 , fig. 7 (not fig. 6).

Remarks. This species differs from $P$. leptoconica in that there are fewer costae (fewer than one per pore), and their crests are pronouncedly convex longitudinally.

\section{Pseudodictyomitra formosa (Squinabol, 1904)} (Pl. 3, Fig. 9)

Dictyomitra formosa Squinabol, 1904, p. 232, pl. 10, fig. 4; Moore, 1973, p. 829, pl. 1, figs. 1-3 (not fig. 4); Pessagno, 1976, p. 51, pl. 8 , figs. $10-12$.

\section{Pseudodictyomitra lanceloti Schaaf, n. sp.}

(Pl. 18, Figs. 9a, b)

Description. Conical shell, tending to be cylindrical or contracted below about the eighth segment. Constrictions between the segments 
pronounced, each associated with a transverse row of pores and a row of alternating dimples (perhaps closed pores) distal to them. Discontinuous costae are present on all segments except the first three or four and the last, which are smooth. Costae are pronounced, but narrow, separated by wide depressions, about half as numerous as the pores in their transverse rows.

Measurements. (Based on 5 specimens from Site 463, between $90, C C$ and $92-1,0-1 \mathrm{~cm}$.) Length of the 6 first segments 140 to 160 $\mu \mathrm{m}$; number of costae per half circumference 7 to 8 .

Type locality. Mid-Pacific Mountains, Site 463 (see Site 463 report, this volume), Core 90.

Type specimens. Holotype: $62-463-89-1,94-95 \mathrm{~cm}$, slide no. 10; England finder no. X 43/2. Paratype: $62-463-90, C C$, SEM negative no. 781109.

Remarks: Pseudodictyomitra lanceloti is distinguished from $P$. carpatica by the latter having wide costae, and more of them on a diameter. This species is named for Dr. Yves Lancelot, in honor of his contributions to the sedimentology of the North Pacific.

\section{Pseudodictyomitra leptoconica (Foreman, 1973) group}

(Pl. 3, Fig. 3; Pl. 18, Figs. 3a, b)

Dictyomitra leptoconica Foreman, 1973b, p. 264, pl. 10, fig. 4, pl. 16, fig. 6 .

Remarks. This name is here applied in a much wider sense than Foreman's, to include all forms having a single costa arising from the margin of each pore, on the proximal as well as on the distal segments. In this species (in contrast to $P$. carpatica), the longitudinal crests of the costae appear concave.

\section{Pseudodictyomitra lilyae (Tan Sin Hok, 1927)}

(Pl. 3, Fig. 8; Pl. 18, Figs. 5a, b)

Dictyomitra lilyae Tan Sin Hok, 1927, p. 55, pl. 10, fig. 83; Riedel and Sanfilippo, 1974, pl. 4, figs. 7-9, pl. 12, fig. 13; Renz, 1974, p. 791 , pl. 8, figs. $1-4$, pl. 11 , fig. 33 .

Remarks. This rather small species of Pseudodictyomitra shows well the characteristic features: segments wider in their upper part, and coarse costae only on the proximal segments.

\section{Pseudodictyomitra lodogaensis Pessagno, 1977}

$$
\text { (Pl. 3, Fig. 5) }
$$

Pseudodictyomitra lodogaensis Pessagno, 1977b, p. 50, pl. 8, figs. 4 , $21,28$.

\section{Pseudodictyomitra vestalensis Pessagno, 1977}

(Pl. 3, Fig. 10)

Pseudodictyomitra vestalensis Pessagno, 1977b, p. 51, pl. 8, figs. 7, 18,24 , pl. 12, fig. 9.

\section{Pseudodictyomitra pseudomacrocephala (Squinabol, 1903)}

(Pl. 24, Figs. 1a, b)

Dictyomitra pseudomacrocephala Squinabol, 1903, p. 139, pl. 10, fig. 2; Cita, 1964, p. 143, pl. 12, figs. 8, 9; Petrushevskaya and Kozlova, 1972 , p. 550 , pl. 2, fig. 5 ; Foreman, 1975, p. 614 , pl. 7 , fig. 10; Pessagno, 1976, p. 53, pl. 3, figs. 2-3.

[?] Dictyomitra sagitafera Aliev, 1961, p. 25, pl. 1, figs. 1-3; Aliev, 1965 , p. 55 , pl. 10 , figs. $2-4$.

Dictyomitra malleola Aliev in Pessagno, 1969, p. 610, pl. 5, fig. A.

Dictyomitra macrocephala Squinabol in Moore, 1973, p. 829, pl. 9, figs. 8, 9; Riedel and Sanfilippo, 1974, pl. 4, figs. 10, 11, pl. 14, fig. 11 .

Dictyomitra sp. Foreman, 1973, p. 264, pl. 14, fig. 16.

\section{Rhopalosyringium majuroensis Schaaf, $\mathrm{n} . \mathbf{s p}$.}

$$
\text { (Pl. 6, Figs. 2, 3; Pl. 23, Fig. 5) }
$$

Rhopalosyringium sp. Foreman, 1971, p. 1682, pl. 3, fig. 9.

[?] Rhopalosyringium sp. A. Moore, 1973, p. 826, pl. 7, fig. 1.

Description. Shell of three segments: hemispherical cephalis, globose thorax, and very fragile cylindrical abdomen. The poreless cephalis, bearing a sturdy three-bladed horn, is rough. An upwarddirected tube which does not protrude emerges on his basis. The thorax is thick-walled and has uniform circular pores in angular frames. Lumbar stricture well-defined by a thick ring, with smooth abdomen narrower than thorax.

Measurements. (Based on 13 specimens from Hole 465A, Section 29-1.) Diameter of cephalis 45 to $60 \mu \mathrm{m}$; diameter of thorax 95 to 120 $\mu \mathrm{m}$; diameter of abdomen 30 to $50 \mu \mathrm{m}$; length of apical horn 36 to 65 $\mu \mathrm{m}$.

Type locality. Hess Rise, Hole $465 \mathrm{~A}$ (see Site 465 report, this volume), Section 29-1.

Type specimens. Holotype: $62-465 \mathrm{~A}-29-1,43-44 \mathrm{~cm}$, slide no. 11 ; England finder no. H 38/3. Paratype: $62-465 \mathrm{~A}-29-1,43-44 \mathrm{~cm}$, SEM negative no. 79325 .

Remarks. This species differs from all other members of the genus by the angular pore frames of its thorax and the thick ring at the lumbar stricture. This species is named for Majuro Atoll (Marshall Islands), embarkation port of Leg 62 .

\section{Saitoum cepeki Schaaf, n. sp.}

(Pl. 6, Fig. 15; Pl. 21, Fig. 8)

Spyrid (?) gen. et sp. indet. Riedel and Sanfilippo, 1974, pl. 3, fig. 4, perhaps 5.

Description. Cephalis almost spherical, with three feet and an apical horn. Cephalic wall hyaline or with small pores. Feet not markedly longer than width of cephalis, circular in section or perhaps slightly bladed in some specimens. Vertical and apical bars free within cephalic cavity, the latter extending as a cylindrical apical horn.

Measurements. (Based on 15 specimens from Site 463, between $84, \mathrm{CC}$ and $90, \mathrm{CC})$. Height of cephalis 60 to $80 \mu \mathrm{m}$; length of horn 10 to $15 \mu \mathrm{m}$; length of feet 30 to $40 \mu \mathrm{m}$.

Type locality. Mid-Pacific Mountains, Site 463 (see Site 463 report, this volume), Core 90 .

Type specimens. Holotype: $62-463-86, \mathrm{CC}$, slide no. 4; England finder no. K 43/3. Paratype: $62-463-87-1,6-7 \mathrm{~cm}$, slide no. 3 ; England finder, no. E $48 / 3$.

Remarks. This species differs from Saitoum pagei Pessagno (1977, p. 98, pl. 12, figs. 11-14) in it less-porous cephalic wall, and smaller, less-bladed feet. Two of the specimens described by Riedel and Sanfilippo (1974) as "Spyrid (?) gen. et sp. indet." are identified with the present species. The cephalic wall tends to become more hyaline, and the feet smaller and more cylindrical. From late Jurassic to Early Cretaceous. This species is named for Dr. Pavel Čepek, in honor of his contributions to the study of Mesozoic nannoplankton.

\section{Sethocapsa orca Foreman, 1975}

$$
\text { (Pl. 26, Figs. 3a, b) }
$$

Sethocapsa (?) orca Foreman, 1975, p. 617, pl. 2J, figs. 1-2, pl. 6, fig. 12.

Remarks. This is the only large Sethocapsa in the Leg 62 material.

\section{Sethocapsa trachyostraca Foreman, 1973}

$$
\text { (Pl. 23, Figs. 1a, b) }
$$

Sethocapsa trachyostraca Foreman, 1973b, p. 268, pl. 12, fig. 4; Riedel and Sanfilippo, 1974, p. 780, pl. 9, figs. 5-7; Foreman, 1975 , p. 617 , pl. 2 J, figs. $3-4$.

Stichocapsa conosphaeroides Rüst in Moore, 1973, p. 827, pl. 4, figs. 5-6.

Sethocapsa uterculus (Parona, 1890)

(P1. 5, Figs. 8a, b; Pl. 26, Figs. 5a, b)

Theocapsa uterculus Parona, 1890, p. 168, pl. 5, fig. 17.

Sethocapsa spp. cf. Theocapsa uterculus Parona in Foreman, 1975, p. 617 , pl. 21 , figs. $21,22$.

\section{Siphocampium? davidi Schaaf, n. sp. \\ (Pl. 5, Fig. 7; Pl. 27, Figs. 10a, b)}

Description. Four-segmented form in which the upper three segments form a rather narrow cone and the fourth is greatly inflated and closed. The cephalis, with a poreless wall, is divided into an upper and a lower chamber, as in Amphipyndax. Some specimens have a short apical horn. Distinct collar and lumbar strictures. Thorax and abdomen with slightly roughened surface, and small pores aligned transversely. Terminal segment with much larger pores regularly quincuncially arranged and hexagonally framed, and thorny surface. 
Measurements. (Based on 14 specimens from Site 463 , between $84, \mathrm{CC}$ and $90, \mathrm{CC}$.) Diameter of cephalis 20 to $22 \mu \mathrm{m}$; diameter of fourth segment 90 to $110 \mu \mathrm{m}$; number of pores per half circumference on the fourth segment 11 to 13 .

Type locality. Mid-Pacific Mountains, Site 463 (see Site 463 report, this volume), Core 90.

Type specimens. Holotype: $62-463-90, \mathrm{CC}$, slide no. 6; England finder no. E 29/1. Paratype: 62-463-90,CC, slide no. 9; England finder no. M 36/2.

Remarks. This species is distinguished from Cyrtocapsa asseni Tan Sin Hok (of which I have examined topotypic material) by the distinctive two-chambered cephalis. Its relationship to Sethocapsa spp. of Foreman $(1975$, p. 617 , pl. 2I, figs. 10-12,14) is uncertain because of the poor preservation of the cephalis in that form. It is uncertain whether this species is related to the type species of Siphocampium (Siphocampium accrescens, Rüst, 1885), but Rüst's form does have a cephalis sufficiently elongated to accommodate two chambers, and that name is used to avoid or postpone the necessity of establishing a new genus. It seems inadvisable at present to extend the concept of Amphipyndax to include closed forms. This species is named for Professor Louis David (Départment des Sciences de la Terre, Lyon) in honor of his contributions to the study of bryozoans, and to my paleontological vocation.

\section{Siphocampium macropora (Rüst, 1888)}

$$
\text { (Pl. 27, Figs. 14a, b) }
$$

? Dicolocapsa macropora Rüst, 1888 , p. 208 , pl. 27 , fig. 25

\section{Siphocampium rutteni (Tan Sin Hok, 1927)}

(Pl. 6, Fig. 5; Pl. 27, Figs. 11a, b)

Stichocapsa rutteni Tan Sin Hok, 1927, p. 71, pl. 15, fig. 133.

? Stichocapsa sp. aff. S. rutteni Tan Sin Hok in Renz, 1974, p. 797, pl. 11 , fig. 6 .

Remarks. The two-chambered cephalis relates this species to the type species of Siphocampium (see remarks under Siphocampium davidi $\mathrm{n} . \mathrm{sp}$.).

\section{Sonelotryma sp.}

$$
\text { (Pl. 21, Figs. 7a, b) }
$$

? Tricolocapsa parvipora Tan Sin Hok in Renz, 1974, pl. 11, fig. 3 (not pl. 6, figs. 8-12).

\section{Sphaeropyle thirencis Schaaf, n. sp.}

$$
\text { (Pl. 17, Figs. 6a, b) }
$$

Description. Three concentric, latticed, spherical shells, connected by approximately 20 radial beams, half of which perhaps extend beyond the surface as short cylindrical spines. A latticed cone, of about the same size as the spherical part, begins at the second shell and extends well beyond the cortical shell.

Measurements. (Based on 4 specimens from Site 463, between 85-1 and 90,CC.) Diameters of the three spheres 10 to $15 \mu \mathrm{m}, 25$ to $34 \mu \mathrm{m}$, 72 to $85 \mu \mathrm{m}$; length of free part of cone 45 to $60 \mu \mathrm{m}$, its width distally 75 to $92 \mu \mathrm{m}$.

Type locality. Mid-Pacific Mountains, Site 463 (see Site 463 report, this volume), Core 89.

Type specimens. Holotype: $62-463-89-1,15-16 \mathrm{~cm}$, slide no. 7 ; England finder no. C 45/1. Paratype: $62-463-90, C C$, slide no. 14; England finder no, M 17/3.

Remarks. This species differs from all others of the genus by the latticed cone. Thirencis, name formed by an arbitrary combination of letters.

\section{Sphaerostylus lanceola (Parona, 1890) group}

(Pl. 7, Fig. 6; Pl. 16, Figs. 5a, b)

Stylosphaera lanceola Parona, 1890, p. 150, pl. 1, fig. 19.

Stylosphaera sp. Parona, 1890 , p. 150, pl. 1, fig. 18; Zhamoida in

Dundo and Zhamoida, 1963, pl. 1, fig. 9; Kling, 1971, p. 1089, pl. 10 , fig. 3 .

Lithatractus sp. Parona and Rovereto, 1895, p. 175, fig. 27 (not fig. 28).

Xiphostylus felsinae Neviani, 1900, p. 649, pl. 9, fig. 7.
Stylatractus ovatus Hinde, 1900, p. 19, pl. 4, figs. 29, 31-33, 36, Moore, 1973, p. 823 , pl. 2, fig. 1.

Stylatractus paronae Hinde, 1900 , p. 18, pl. 4, fig. 34.

Stylosphaera squinaboli Tan Sin Hok, 1927, p. 35, pl. 6, figs. 9a-d. Radiolarian (gen. and sp. indet.) Turner, 1965, p. 394, pl. 52, figs. 14, 15.

Spumellariinid Pessagno, 1969, p. 610, pl. 4, fig. D.

Sphaerostylus lanceola (Parona) group in Foreman, 1973b, p. 258, pl. 1, figs. 7-11; Foreman, 1975, p. 609, pl. 2E, figs. 3-6.

Sphaerostylus lanceola (Parona) in Riedel and Sanfilippo, 1974, pl. 1, figs. $1-3$.

Pantanellium corriganensis Pessagno, 1977b, p. 33, pl. 3, figs. 5, 6.

\section{Spongobrachium? sp.}

(Pl. 12, Fig. 3)

Description. Discoidal spongy form, subcircular to elliptical in outline, with a distinct central latticed sphere.

Measurements. Total diameter 120 to $190 \mu \mathrm{m}$; diameter of internal sphere 45 to $55 \mu \mathrm{m}$.

Remarks. It is unlikely that this form is related to the type species of Spongobrachium (Spongodiscus ellipticus Haeckel, 1862), but no more suitable generic name is available, and it would be premature to define a new genus.

\section{Spongocapsula? zamoraensis Pessagno, 1976}

(P1. 24, Figs. 2a, b)

Stichomitra (?) zamoraensis Pessagno, 1976, p. 54, pl. 3, figs. 7-9. Spongocapsula zamoraenis Pessagno, 1977b, p. 53, pl. 9, figs. 5, 16. Remarks. Cephalis and thorax are small and not spongy.

\section{Spongocyclia trachodes Renz, 1974}

(Pl. 12, Fig. 8)

Spongocyclia trachodes Renz, 1974, p. 796, pl. 4, figs. 1-4, pl. 10, fig. 13 (not fig. 7).

\section{Spongodiscus americanus Kozlova, 1966}

(Pl. 8, Fig. 10)

Spongodiscus americanus Kozlova and Gorbovets, 1966, p. 88, pl. 14, figs. 1-2.

not Spongodiscus sp. cf. S. americanus Kozlova in Renz, 1974, p. 796, pl. 3 , fig. 12 , pl. 10 , fig. 6 .

Remarks. The Cretaceous species is smaller than the original Cenozoic holotype.

\section{Spongodiscus misele Schaaf, n. sp.}

$$
\text { (P1. 17, Figs. 3a, b) }
$$

Description. Spongy, approximately discoidal form, a part of which shows concentric and radial structure near one edge. From this part radiate several spines, two of which commonly are bladed, much more prominent than the others. No indication of a pylome, Many specimens are incomplete in the part away from the spines.

Measurements. (Based on 4 specimens from Site 463, between 70-2 and 73-1.) Total diameter of almost complete specimens 280 to 320 $\mu \mathrm{m}$; length of large spines 80 to $120 \mu \mathrm{m}$.

Type locality. Mid-Pacific Mountains, Site 463 (see Site 463 report, this volume), Core 73 .

Type specimens. Holotype: $62-463-73-1,18-19 \mathrm{~cm}$, slide no. 4; England finder no. U 34/1. Paratype: $62-463-75-1,21-22 \mathrm{~cm}$, slide no. 34; England finder no. X 36/3.

Remarks. This species differs from all others of the genus by the pronounced eccentricity of the part with concentric and radial structure. Misele, name formed by an arbitrary combination of letters.

Spongodiscus renillaeformis Campbell and Clark, 1944

(Pl. 8, Figs. 4, 5, 8; Pl. 13, Fig. 9; Pl. 15, Fig. 1)

Spongodiscus renillaeformis Campbell and Clark, 1944, p. 18, pl. 6, figs. $5,6,8,10$.

Spongodiscus impressus Lipman in Kozlova and Gorbovets, 1966, p. 87 , pl. 4 , figs. 8,9 .

Remarks. We take under this name all the spongy discoidal tests with an elevated central area and a truncated outline. 
?Spongodruppa cocos Rüst, 1898

(Pl. 6, Fig. 13; Pl. 15, Figs. 4a, b)

Spongodruppa cocos Rüst, 1898, p. 19, pl. 6, fig. 5

\section{Spongopyle ecleptos Renz, 1974}

$$
\text { (Pl. 17, Figs. 2a, b, 9) }
$$

Spongopyle ecleptos Renz, 1974, p. 796, pl. 3, figs. 2-6, pl. 10, fig. 14 Remarks. This name is here used in a much broader sense than by Renz (1974), to include also forms with round or three-bladed spines around the disk.

\section{Spongopyle insolita Kozlova, 1966, group}

$$
\text { (Pl. 17, Figs. 7, 8) }
$$

Spongopyle insolita Kozlova and Gorbovets, 1966, p. 91, pl. 4, figs. 11a-b; Riedel and Sanfilippo, 1974, p. 780, pl. 2, figs. 7, 8, 11 (not figs. 9, 10), pl. 14, fig. 4 .

Remarks. There is a tendency for the size of the skeleton and the amount of spongy material to decrease from the Barremian to the Cenomanian.

\section{Spongosaturnalis horridus (Squinabol, 1903) group}

$$
\text { (Pl. 16, Fig. 4) }
$$

Acanthocircus horridus Squinabol, 1903, p. 125, pl. 9, fig. 3 Saturnalis polymorphus Squinabol, 1914 (invalid name), p. 293.

Spongosaturnalis polymorphus (Squinabol) in Moore, 1973, p. 824 pl. 6 , figs. 4,6 .

Spongosaturnalis horridus (Squinabol) in Foreman, 1975, p. 610, pl. 2C, fig. 1, pl, 4, fig. 3 .

Spongosaturnalis (?) spp. Foreman, 1975, p. 612, pl. 1C, figs. 3-10, pl. $2 \mathrm{C}$, figs. 2-6.

Remarks. Because members of this genus are not common nor well preserved in the Leg 62 material, this species-group name is used for all forms with about 10 to 15 external spines.

\section{Staurocyclia martini (Rüst, 1898)}

$$
\text { (Pl. 11, Figs. 2a, b) }
$$

Staurocyclia martini Rüst, 1898, p. 21, pl. 6, fig. 11.

Remarks. Specimens observed here agree well with the descriptions of Rüst, except for the three-bladed arms.

\section{Staurosphaera septemporata Parona, 1890}

(Pl. 7, Figs. 8a, b; Pl. 16, Figs. 10a, b)

Staurosphaera septemporata Parona, 1890, p. 151, pl. 2, figs. 4, 5; Cita and Pasquare, 1959, p. 398, fig. 3, no. 7; Moore, 1973, p. 824 , pl. 2, fig. 2, Foreman, 1973, p. 259, pl. 3, fig. 4; Riedel and Sanfilippo, 1974, p. 780, pl. 1, figs. 6-8; Foreman, 1975, p. 609, pl. $2 \mathrm{E}$, fig. 7 , pl. 3 , fig. 6 .

Cecrops septemporatus (Parona) in Pessagno, 1977b, p. 33, pl. 3, fig. 11.

\section{?Stephanastrum inflexum Rüst, 1898}

$$
\text { (Pl. 14, Figs. 4a, b) }
$$

Stephanastrum inflexum Rüst, 1898, p. 32, pl. 11, fig. 2.

\section{Stichocapsa cribata Hinde, 1900}

(Pl. 6, Fig. 4; Pl. 25, Fig. 6)

Stichocapsa cribata Hinde, 1900, p. 43, pl. 4, fig. 39; Moore, 1973, p. 827 , pl. 4 , figs. 1-2.

\section{Stichocapsa decora Rüst, 1885}

(Pl. 27, Figs. 13a, b)

Stichocapsa decora Rüst, 1885, p. 319, pl. 17, fig. 3.

\section{Stichocapsa sp. cf. S. decora Rüst, 1885}

(Pl. 27, Figs. 12a, b)

Stichocapsa decora Rüst, 1885, p. 319, pl. 17, fig. 3.

Remarks. This form is very similar in shape and in size to Stichocapsa decora, but the last post-abdominal segment bears small pores.

\section{Stichocapsa pseudodecora Tan Sin Hok, 1927}

(Pl. 27, Figs. 7a, b)

Stichocapsa pseudodecora Tan Sin Hok, 1927, p. 72, pl. 16, fig. 137, (not Renz, 1974, pl. 11, fig. 29).

\section{Stichocapsa pseudopentacola Tan Sin Hok, 1927 \\ (Pl. 27, Figs. 4a, b)}

Stichocapsa pseudopentacola Tan Sin Hok, 1927, p. 72, pl. 16, fig. 136.

\section{Stichomitra asymbatos Foreman, 1968}

(Pl. 22, Figs. 6a, b)

Stichomitra asymbatos Foreman, 1968, p. 73, pl. 8, figs. 10a-c; Riedel and Sanfilippo, 1974, p. 780, pl. 10, figs. 1-4 (not figs. 5-7), pl. 15, fig. 5; Foreman, 1978, p. 748, pl. 4, fig. 15.

Stylochlamyum? sp. group

(Pl. 12, Fig. 1; Pl. 16, Figs. 6a, b)

Description. A coarsely spiral disk, with a prominent central sphere in some specimens, surrounded by an irregular spongy zone. In most specimens, the original skeleton seems to have been filled and replaced, and in this process the coarser skeletal elements may have been preferentially emphasized. It is now difficult to reconstruct the original distribution of the skeletal elements and the spaces between them.

Measurements. Diameter of spiral portion 280 to $340 \mu \mathrm{m}$.

Remarks. It is unlikely that these forms are related to the type species of Stylochlamyum, and that name is used as a matter of temporary convenience. They are probably related to the forms recorded by Renz (1974, pl. 10, fig. 7) as species of Spongocyclia.

\section{Stylosphaera macrostyla Rüst, 1888}

(P1. 14, Fig. 2)

Stylosphaera macrostyla Rüst, 1888, p. 193, pl. 22, fig. 12.

\section{Thanarla karpoffae Schaaf, n. sp.} (Pl. 21, Fig. 3)

Description. Four upper segments forming a conical section with indistinct external strictures, and a fifth forming a subcylindrical lower section. The four first segments have a thick wall and are commonly preserved, while the thin-walled fifth segment is rarely seen. Surface ornamented by continuous ribs separating single rows of pores which are rather regularly spaced on the third and fourth segments, and less regular and sometimes longitudinally elongated on the fifth. Terminal feet not observed.

Measurements. (Based on 5 specimens from Site 463, between 89-1 and $90, C C$.) Length of the four upper segments 120 to $140 \mu \mathrm{m}$; diameter of fifth segment 50 to $65 \mu \mathrm{m} ; 5$ to 6 rows of pores at fourth segment.

Type locality. Mid-Pacific Mountains, Site 463 (see Site 463 report, this volume), $90, \mathrm{CC}$.

Type specimens. Holotype: $62-463-90, C C$, slide no. 10; England finder no. U 26/0. Paratype: 62-463-90,CC, slide no. 7; England finder no. B 35/2.

Remarks. Distinguished from other members of the genus by the pronounced difference between the fourth and the fifth segments. This species is named for Anne-Marie Karpoff (Institut de Géologie, Strasbourg), in honor of her contributions to the study of the geochemistry of marine sediments.

\section{Thanarla pulchra (Squinabol, 1904)}

(Pl. 4, Fig. 10; Pl. 19, Figs. 7a, b)

Sethamphora pulchra Squinabol, 1904, p. 213, pl. 5, fig. 8; Moore, 1973 , p. 826 , pl. 3, figs. 5, 6 (not fig. 4); Riedel and Sanfilippo, 1974 , pl. 13, fig. 5 .

Dictyomitra pulchra (Squinabol) in Dumitrică, 1975, p. 87, text-fig. 2, fig. 7.

Thanarla pulchra (Squinabol) in Pessagno, 1977b, p. 46, pl. 7, figs. 7, $21,26$.

Remarks. No specimens were found with the external change in contour characteristic of Thanarla elegantissima (Cita, 1964). 
Theocampe sp. cf. T. salillum Foreman, 1971 (Pl. 24, Fig. 7)

Theocampe salillum Foreman, 1971, p. 1678, pl. 4, fig. 5; Moore, 1973 , p. 826 , pl. 11, figs. 1, 2; Foreman, 1973, pl. 15, fig. 12; Foreman, 1975 , p. 613 , pl. 1 F, fig. 8 , pl. 6 , fig. 4 .

\section{Theocampe vanderhoofi Campbell and Clark, 1944} (P1. 24, Figs. 5a, b, 11a, b)

Theocampe vanderhoofi Campbell and Clark, 1944, p. 34, pl. 7, fig. 19.

Theocapsa laevis Tan Sin Hok, 1927

$$
\text { (Pl. 27, Fig. 9) }
$$

Theocapsa laevis Tan Sin Hok, 1927, p. 46, pl. 8, fig. 49.

\section{Theocapsomma ancus Foreman, 1968}

(Pl. 24, Figs. 4, 9a, b)

Theocapsomma ancus Foreman, 1968, p. 32, pl. 4, fig. 3.

Diacanthopcapsa cf. ancus (Foreman) in Dumitrică, 1970, p. 64, pl. 6, figs. $35 \mathrm{a}-\mathrm{b}$, pl. 7 , fig. 40 , pl. 20 , fig. 125 .

\section{Theocorys antiqua Squinabol, 1903}

(Pl. 24, Figs. 10a, b)

Theocorys antiqua Squinabol, 1903, p. 135, pl. 8, fig. 25.

Theocorys sp. aff. T. antiqua Squinabol in Renz, 1974, p. 798, pl. 6, figs. 4-7, pl. 11, fig. 4 .

\section{Theocorys renzae Schaaf n. sp.}

(Pl. 5, Figs. 13a-c; P1. 27, Figs. 1a, b)

Description. Four-segmented form with small cephalis, without apical horn, poreless. Small thorax and abdomen truncate-conical in shape. Post-abdominal segment very inflated, subspherical to ellipsoidal. Very contracted aperture with protruding rim. Small pores regularly disposed in intersecting diagonal rows.

Measurements. (Based on 7 specimens from Site 463, between 89-1, 105-107 $\mathrm{cm}$ and 90,CC.) Height of shell 140 to $190 \mu \mathrm{m}$; diameter of cephalis 10 to $15 \mu \mathrm{m}$; height of thorax 10 to $13 \mu \mathrm{m}$; height of abdomen 18 to $22 \mu \mathrm{m}$; breadth of post-abdominal segment 100 to 130 $\mu \mathrm{m}$.

Type locality. Mid-Pacific Mountains, Site 463 (see Site 463 report, this volume), Cores 89 and 90 .

Type specimens. Holotype: 62-463-90,CC, slide no. 9; England finder no. W 44/4. Paratype: $62-463-89-1,105-106 \mathrm{~cm}$; SEM negative nos. 781061, 781062, 781158 .

Remarks. This species differs from $T$. antiqua Squinabol in having smaller pores not aligned in longitudinal rows, and a four-segmented shell. This species is named for Dr. G. W. Renz-Killmar, in honor of her contributions to the study of Mesozoic and Cenozoic Radiolaria.

\section{Triactoma hybum Foreman, 1975}

(Pl. 12, Fig. 7)

Triactoma sp. cf. $T$. echoides Foreman, 1973b, pl. 3, fig. 2 (not fig. 3).

Triactoma hybum Foreman, 1975, p. 609, pl. 2F, figs. 6, 7, pl. 3, figs . $7,9$.

\section{Tripocalpis ellyae Tan Sin Hok, 1927}

$$
\text { (P1. 23, Figs. 6a, b) }
$$

Tripocalpis ellyae Tan Sin Hok, 1927, p. 38, pl. 7, fig. 18; (not Renz, 1974 , pl. 5, figs. 18,19 , pl. 11 , fig. 10).

\section{?Tripocyclia trigonum Rüst, 1885}

(Pl. 13, Fig. 3)

Tripocyclia trigonum Rüst, 1885 , p. 293 , pl. 30 , fig. 3 .

\section{Ultranapora durhami Pessagno, 1977}

$$
\text { (Pl. 23, Figs. 8a, b) }
$$

Ultranapora durhami Pessagno, 1977b, p. 39, pl. 5, figs. 1-3, 13, 14, 19 , pl. 12 , fig. 4
Tripocalpis ellyae Tan Sin Hok in Renz 1974, p. 798, pl. 5, figs. $18-19$, pl. 11, fig. 10.

\section{Ultranapora spinifera Pessagno, 1977}

(Pl. 25, Figs. 9a, b)

Tripilidium (?) deudrocanthos Squinabol in Pessagno, 1976, p. 55, pl. 3 , fig. 1 .

Ultranapora spinifera Pessagno, 1977b, p. 39, pl. 5, figs. 5, 11, 12, pl. 12 , fig. 7 .

\section{Williriedellum carpathicum Dumitrică, 1970}

(Pl. 1, Figs. 2a, b)

Williriedellum carpathicum Dumitrică, 1970 , p. 70 , pl. 9, figs. 56a-b, 57-59, pl. 10, fig. 61 .

Remarks. This rare species is larger than Dumitrică's original holotype.

\section{Williriedellum gilkeyi Dumitrică, 1972}

(Pl. 2, Figs. 6a-c)

Williriedellum (?) gilkeyi Dumitrică, 1972, p. 841, pl. 3, figs. 4, 6, pl. 4 , figs. 1,2

Remarks. The lack of thoracic apophyses confirms the doubtful generic assignment of Dumitrică's species. The less closely packed pores are perhaps the attribute of an ancestor form, linking $W$. crystallinum Dumitricǎ, from the upper Callovian-Oxfordian, and W. gilkeyi, from the Albian of Leg 13.

Williriedellum peterschmittae Schaaf, n. sp.

(Pl. 1, Figs. 3a, b; Pl. 9, Figs. 3a, b)

Hemicryptocapsa (?) nodosa (Tan Sin Hok) in Dumitrică, 1972, p. 841 , pl. 1 , fig. 6 , pl. 2 , figs. $1,2$.

Description. Nodose, spherical form with protruding cephalis. Small, apically pointed but hornless cephalis, with a few small pores. Thorax more than hemispherical within abdominal wall and cavity, with a large aperture and no spine. Abdomen thick-walled, with a small mouth surrounded by a distinct peristome. Abdominal wall pronouncedly tuberculate, with small pores regularly arranged over the entire surface; on each node, one central pore is surrounded by six others.

Measurements. (Based on 23 specimens from Site 463, between $84, \mathrm{CC}$ and 90,CC.) Diameter of shell 120 to $145 \mu \mathrm{m}$; diameter of cephalis 8 to $12 \mu \mathrm{m}$; number of abdominal nodes per half circumference 8 to 10 .

Type locality. Mid-Pacific Mountains, Site 463 (see Site 463 report, this volume), $90, \mathrm{CC}$.

Type specimens. Holotype: $62-463-89-1,105-106 \mathrm{~cm}$, slide no. 3; England finder no. H 35/2. Paratypes: $62-463-90, C C$, slide no. 7; England finder no. B 35/4; 62-463-90,CC, SEM negative nos. 781055, 781056.

Remarks. This species differs from Tricolocapsa nodosa Tan Sin Hok, with which it occurs in Rotti's sample 149, by the more-regular abdominal pores and the sunken thorax. This species is named for Iréne Peterschmitt, to thank her for her care in taking the scanning electron micrographs.

Xitus alievi (Foreman, 1973)

(Pl. 5, Figs. 4a, b; Pl. 19, Figs. 1a, b, 8a, b)

Dictyomitra alievi Foreman, 1973b, p. 263, pl. 9, fig. 10, pl. 16, fig. 4; Foreman, 1975 , p. 613 , pl. $2 \mathrm{H}$, figs. 8,9 , pl. 7 , fig. 2.

Xitus plenus Pessagno, 1977b, p. 55, pl. 9, figs. 15, 21, 22, 26, pl. 12, fig. 15 .

Remarks. This species is distinguished from Xitus spicularius by its narrower segments, fewer transverse rows of pores per segment, and, in the scanning electron micrographs, by a lacy rather than tuberculate surface. Upward in the section the wall becomes thinner.

Xitus spicularius (Aliev, 1961)

(Pl. 4, Fig. 11; Pl. 5, Figs. 12a, b; Pl. 19, Figs. 2a, b)

Dictyomitra spicularia Aliev, 1961, p. 34, pl. 2, figs. 1, 2; Aliev, 1965 , p. 39, pl. 6 , fig. 9 
Dictyomitra sp. cf. D. spicularia Aliev in Foreman, 1973b, p. 264, pl. 9 , figs. 8,9 .

Xitus spicularius (Aliev) in Pessagno, 1977b, p. 56, pl. 9, fig. 7, pl. 10, fig. 5 .

Remarks. For characters distinguishing this species from $X$. alievi, see under that name. Upward in the section, the shape tends to become more cylindrical.

\section{Xitus sp. cf. $X$. spicularius (Aliev, 1961) (Pl. 4, Fig. 12)}

Remarks. This species differs from $X$. spicularius by having a slender test and less-massive tubercules.

\section{Xitus vermiculatus (Renz, 1974)}

$$
\text { (Pl. 19, Figs. 6a, b) }
$$

Eucyrtidium vermiculatum Renz, 1974, p. 792, pl. 8, figs. 17-19, pl. 11 , fig. 22.

Xitus spineus Pessagno, 1977b, p. 56, pl. 10, figs. 3, 12, 16, 20, pl. 12, fig. 13.

Remarks. Xitus vermiculatus differs from $X$. alievi by the lessnodose surface, five irregular rows of pores (rather than three), and thinner wall. It differs from Stichomitra asymbatos Foreman by the small apical spine and the irregular rows of pores.

Xitus sp. A.

(Pl. 5, Figs. 9a, b; Pl. 18, Figs. 7a, b)

Remarks. This form, very similar to $X$. alievi, is narrower and more cylindrical, with a larger, round cephalis.

\section{ACKNOWLEDGMENTS}

The analytical part of this work was performed with the technical and financial support of the Centre de Sédimentologie et Géochimie de la Surface of the CNRS and the Institut de Géologie et de Minéralogie, in Strasbourg.

I am grateful to W. R. Riedel and A. Sanfilippo for their hospitality and cooperation at the Scripps Institution of Oceanography during six weeks in the Spring of 1979. Discussions with H. R. Thierstein, A. Budai, P. Doyle, and E. Vincent were very fruitful.

I. Peterschmitt operated the SEM, J. Grüner carried out photographic work, and the onerous tasks of typing were competently performed by C. Romon and G. Pagand.

I am grateful also to P. Baumgartner, Basel, for reviewing the draft of this paper, to A. M. Karpoff and M. Hoffert for suggestions.

\section{REFERENCES}

Aliev, Kh. Sh. 1961. Novye vidy radioliarii nizhnego melo severo-vostochnogo Azerbaidzhana [New radiolarian species of the Lower Cretaceous of northeastern Azerbaidzhan]. Uch. Zap. Izd. Azerbaidz. Univ. (Ser. Geol.-Geogr.), 2:25-38.

1965. Radioliarii nizhnemelovykh otozhenii severo-vostochnogo Azerbaidzhana i ikh stratigraficheskoe znachenie [Radiolarians of the Lower Cretaceous deposits of northeastern Azerbaidzhan and their stratigraphic significance]. Izd. Akad. Nauk Azerbaidz. SSR, 3-124.

, 1967. Novye vidy radioliarii valanzhinskogo i albskogo iarusov severo-vostochnogo Azerbaidzhana [New species of Radiolaria of the Valanginian and Albian stages of northeastern Azerbaidzhan]. In Melovye Otlozheniia Vostochnogo Kavkaza $i$ Prilegayuschikh oblastei [Cretaceous Deposits of the Eastern Caucasus and adjacent regions]. Biostrat. Paleogeogr. Akad. Nauk SSSR Inst. Geol., pp. 23-30.

Baumgartner, P. O., in press. Late Jurassic and Early Cretaceous Hagiastridae and Patulibracchidae (Radiolaria) from the Argolis Peninsula (Peloponnesus, Greece). Micropaleont.

Campbell, A. S., and Clark, B. L., 1944. Radiolaria from Upper Cretaceous of Middle California. Geol. Soc. Am. Spec. Papers, 57:1-61.

Cita, M. B. S., 1964. Ricerche micropaleontolgiche e stratigrafiche sui sedimenti pelagici del Giurassico superiore e del Cretaceo inferiore nella catena del Monte Baldo. Riv. Ital. Paleontol. Strat. Mem., 10:1-182.
Cita, M. B., and Pasquaré, G., 1959. Osservazioni micropaleontogiche sul Cretaceo delle Dolomiti. Riv. Ital. Paleontol. Stratigr., 65(4):385-442.

DelFlandre, G., 1953. Radiolaires fossiles. In Grassé, P. P. (Ed.), Traité de Zoologie (Vol. 1): Paris (Masson), 389-436.

Dumitrică, P., 1970. Cryptocephalic and cryptothoracic Nassellaria in some Mesozoic deposits of Romania. Rev. Roum. Geol. Geophys. Geogr. (Ser. Geol.), 14:45-124.

1972. Cretaceous and Quaternary Radiolaria in deep sea sediments from the north-west Atlantic Ocean and Mediterranean Sea. In Ryan, W. B. F., Hsü, K. J., et al., Init. Repts. DSDP, 13, Part 2: Washington (U.S. Govt. Printing Office), 829-901.

1975. Cenomanian Radiolaria at Podul Dimbovitei (Excursion B), in Micropaleontological Guide to the Mesozoic and Tertiary of the Romanian Carpathians (14th European Micropaleontological Colloquium), pp. 87-89.

Dundo, O. P., and Zhamoida, A. I., 1963. Stratigrafiya mezozoiskikh otlozhenii basseina $\mathrm{r}$. belikoi i kharakternyi kompleks valanzhinzkikh radiolyarii. Geol. Koryak. Nagorya, 64-86.

Ehrenberg, C. G., 1838. Über die Bildung der Kreidefelsen und des Kreidemergels durch unsichtbare Organismen. Abhandl. Akad. Wiss. Berlin, Jahrg. 1838, 59-147.

1847. Über die mikroskopischen kieselschaligen Polycystinen als mächtige Gebirgsmasse von Barbados. Kgl. Preuss. Akad. Wiss. Berlin, Monatsber., Jahrg. 1847, 40-60.

, 1875. Fortsetzung der mikrogeologischen Studien als Gesamt-Uebersicht der mikroskopischen Palaontologie gleichartig analysierter Gebirgsarten der Erde, mit spezieller Rucksicht auf den Polycystinen-Mergel von Barbados. Preuss. Akad. Wiss. Berlin, Jahrg. 1875, 1-226.

Fischli, H., 1916. Beitrag zur Kenntnis der fossilen Radiolarien in der Riginagelfluh. Mitt. Naturwiss. Ges. Winterthur, 11:44-37.

Foreman, H. P., 1968. Upper Maestrichtian Radiolaria of California. Spec. Papers Palaentol., 3:1-82.

1971. Cretaceous Radiolaria. In Winterer, E. L., Riedel, W. R., et al., Init. Repts. DSDP, 7, Pt. 2: Washington (U.S. Govt. Printing Office), 1673-1693.

1973a. Radiolaria of Leg 10 with systematics and ranges of the families Amphipyndacidae, Artostrobiidae, and Theoperidae. In Worzel, J. L., Bryant, W., et al., Init. Repts. DSDP, 10: Washington (U.S. Govt. Printing Office), 407-474.

, 1973b. Radiolaria from DSDP Leg 20. In Heezen, B. C., MacGregor, I. D., et al., Init. Repts. DSDP, 20: Washington (U.S. Govt. Printing Office), 249-305.

1975. Radiolaria from the North Pacific, Deep Sea Drilling Project, Leg 32. In Larson, R. L., Moberley, R., et al., Init. Repts. DSDP, 32: Washington (U.S. Govt. Printing Office), 579676.

1977. Mesozoic Radiolaria in the Atlantic ocean of the Northwest coast of Africa, Deep Sea Drilling Project, Leg 41. In Lancelot, Y., Scibold, E., et al., Init. Repts. DSDP, 41: Washington (U.S. Govt. Printing Office), 739-761.

Haeckel, E., 1862. Die Radiolarien (Rhizopoda Radiolaria), eine Monographie: Berlin (Reimer), pp. 1-572.

1881. Entwurf eines Radiolarien-Systems auf Grund von Studien der Challenger-Radiolarien. Jena. Z. Med. Naturwiss., $15: 418-472$

, 1887. Report on the Radiolaria collected by H.M.S. Challenger during the years 1873-1876. Rept. Voyage Challenger, Zool., 18.

Haecker, V., 1908. Tiefsee-Radiolarien. Wiss. Ergen. Deutschen Tiefsee Exped. (Valdivia), 14:337-476.

Hertwig, R., 1879. Der Organismus der Radiolarien: Jena (Fischer) pp. $1-149$.

Hinde, G. J., 1900. Description of fossil Radiolaria from the rocks of Central Borneo. In Molengraaff, G. A. F. (Ed.), BorneoExpedite: Geologische Verkenningstochten in Centraal-Borneo (1893-1894): Leiden (Brill).

Kling, S. A., 1971. Radiolaria. In Fischer, A. G., Heezen, B. C., et al., Init. Repts. DSDP, 6: Washington (U.S. Govt. Printing Office), 1069-1117.

Kozlova, G. E., and Gorbovets, A. N., 1966. Radiolyarii verkhnemelovykh i verkhneeotsenovykh otlozhenii Zapadno-Sibirskoi Niz- 
mennosti. Tr. Vses. Neft. Nauchn-Issled. Geol. Inst. (VNIGRI), 248:1-118.

Lipman, R., 1952. Materialy morphographicheskomu izucheniyu radiolyarii verkhnemelovykh otlozhenii russkoi platformy. Paleontol. Strat. Vses. Nauchn-Issled. Geol. Inst., (VSEGEI), 24-51. 1960. Radiolaria. In Stratigrafiya i Fauna Melovykh Otlozhenii Zapadno-Sibirskoi Nizmennosti. N. S., Tr. Vses NauchnIssled. Geol. Inst. (VSEGEI) (new ser.), 29:124-134.

1962. Pozdnemelovye radiolyarii Zapadno-Sibirskoi Nizmennosti i Turgaiskogo progiba. Vses. Nauch.-Issled. Geol. Inst., Tr., 77: 271-323.

Lozyniak, P. Yu., 1969. Radiolyarii nizhnemelovykh otlozhenii Ukrainskikh Karpat. In Vyalov, O. S. (Ed.), Iskopaemye $i$ Sovremennye Radiolyarii: L'vov (L'vovskoe Geol. O-vo.), pp. 29-41.

Moore, T. C., Jr., 1973. Radiolaria from Leg 17 of the Deep Sea Drilling Project. In Winterer, E. L., Ewing, J. I., et al., Init. Repts. DSDP, 17: Washington (U.S. Govt. Printing Office), 797-869.

Neviani, A., 1900. Supplemento alla fauna a radiolari delle rocce mesozoiche del Bolognese. Boll. Soc. Geol. Ital., 19:645-671.

Parona, C. F., 1890. Radiolarie nei noduli selciosi del calcare giurese di Cittiglio presso Laveno. Boll. Soc. Geol. Ital., 9:1-46.

Parona, C. F., and Rovereto, G., 1895. Diaspri permiani a radiolarie di Montenotte (Liguria Occidentale). Atti R. Accad. Sci. Torino, Cl. Sci. fis., mat. natur., 31:167-181.

Pessagno, E. A., Jr., 1963. Upper Cretaceous Radiolaria from Puerto Rico. Micropaleont., 9:197-214.

1969. Mesozoic planktonic Foraminifera and Radiolaria. In Ewing, M., Worzel, J. L., et al., Init. Repts. DSDP, 1: Washington (U.S. Govt. Printing Office), 607-621.

, 1971. Jurassic and Cretaceous Hagiastridae from the Blake-Bahama Basin (Site 5A, JOIDES Leg 1) and the Great Valley sequence, California Coast Ranges. Bull. Am. Paleont., 60:1-83.

1972. Pt. I: The Phaseliformidae, new family, and other Spongodiscacae from the Upper Cretaceous portion of the Great Valley sequence. Pt. 2: Pseudaulophacidae Riedel from the Cretaceous of California and the Blake-Bahama Basin (JOIDES Leg 1). Bull. Am. Paleont., 61:261-328.

1973. Upper Cretaceous Spumellariina from the Great Valley sequence, California Coast Ranges. Bull. Am. Paleont., 63: 49-102.

, 1976. Radiolarian zonation and stratigraphy of Upper Cretaceous portion of the Great Valley sequence, California Coast Ranges. Micropaleont. Spec. Paper, 2:1-95.

1977a. Upper Jurassic Radiolaria and radiolarian biostratigraphy of the California Coast Ranges. Micropaleont., 23: 56-113.

1977b. Lower Cretaceous Radiolarian biostratigraphy of the Great Valley sequence and Franciscan complex, California Coast Ranges. Cushman Found. Foraminiferal Res. Spec. Pub., 15:1-87.

Petrushevskaya, M. G., 1971. On the natural system of polycystine Radiolaria (Class Sarcodina). In Farinacci, A. (Ed.), Second Plankt. Conf. Proc., Roma 1970. (Vol. 2), 981-991.

Petrushevskaya, M. G. and Kozlova, G. E., 1972. Radiolaria: Leg 14, Deep Sea Drilling Project. In Hayes, D. E., Pimm, A. C., et al., Init. Repts. DSDP, 14: Washington (U.S. Govt. Printing Office), 495-648.

Renz, G. W., 1974. Radiolaria from Leg 27 of the Deep Sea Drilling Project, In Heirtzler, J. R., Veevers, J. J., et al., Init. Repts. $D S D P, 27$ : Washington (U.S. Govt. Printing Office), 769-841.

Riedel, W. R., 1967a. Class Actinopoda. Protoza. In Harland, W. B. (Ed.), The Fossil Record, a Symposium with Documentation:London (Geol. Soc.), pp. 291-298.

1967b. Some new families of Radiolaria. Proc. Geol. Soc. London., no. 1640:148-149. 1971. Systematic classification of polycystine Radiolaria. In Funnel, B. M., and Riedel, W. R. (Eds.), The Micropaleontology of Oceans: Cambridge (Cambridge Univ. Press), pp. 649661.

Riedel, W. R., and Sanfilippo, A., 1970. Radiolaria, Leg 4, Deep Sea Drilling Project. In Bader, R. G., Gerard, R. D., et al., Init. Repts. DSDP, 4: Washington (U.S. Govt. Printing Office), 503-575.

1971. Cenozoic Radiolaria from the western tropical Pacific, Leg 7. In Winterer, E. L., Riedel, W. R., et al., Init. Repts. DSDP, 7, Pt. 2: Washington (U.S. Govt. Printing Office), 1529-1672.

1974. Radiolaria from the southern Indian Ocean, DSDP Leg 26. In Davies, T. A., Luyendyk, B. P., et al., Init. Repts. $D S D P, 26:$ Washington (U.S. Govt. Printing Office), 771-814.

Rüst, D. 1885. Beiträge zur Kenntniss der fossilen Radiolarien aus Gesteinen des Jura. Palaeontogr., 31:269-321.

1888. Beiträge zur Kenntniss der fossilen Radiolarien aus Gesteinen der Kreide. Palaeontogr., 34:181-213.

, 1898. Neue Beiträge zur Kenntniss der fossilen Radiolarien aus Gesteinen des Jura und der Kreide. Palaeontogr., 45:1-67.

Squinabol, S., 1903. Le Radiolarie dei noduli selciosi nella scaglia degli Euganei. Riv. Ital. Paleont., 9:105.

1904. Radiolarie cretacee degli Eugani. Atti Mem. Reale Accad. Sci. Lett. Arti Padova., 20:171-244.

1914. Contributo alla conoscenza dei Radiolarii fossili del Veneto. Appendice: di un genere di Radiolari caratteristico del Secondario. Geol. Univ. Padova, Mem., 2:249.

Tan Sin Hok, 1927. Over de samenstelling en het onstaan van krijt-en mergelgesteenten van de Molukken. Jaar. Mijn. Ned.-Vost-Indie, Jahrg. 1926, Verhandl., (part 3), 5-165.

Turner, J., 1965. Upper Jurassic and Lower Cretaceous microfossils from the Hautes-Alpes. Paleont., 8(3):391.

Zhamoida, A. I., 1969. Pervye rezul'taty izuchenia mezozoiskikh radioliarii Sakhalina [First results of the study of Mesozoic Radiolaria of Sakhalin]. In Vialov, O. C. (Ed.) Iskopaemye i Sovremennye Radioliarii [Fossil and Recent Radiolarians] L'vov (L'vov Geol. O-vo), pp. 17-28.

1972. Biostratigrafiya mezozoiskikh kremnistykh tolshch vostoka SSSR na osnove izucheniya radioliarii [Biostratigraphy of the Mesozoic siliceous series of the eastern USSR by study of Radiolarian]. Tr. VSEGEI, (new ser.), 183:243.

Zhamoida, A. I., Kovtunovich, Yu. M., and Saviiki, V. O., 1968. Kompleksy mezozoiskikh radiolarii vostochno-Sakhalinskikh gor [Complex of Mesozoic Radiolaria in the eastern Sakhalin mountains]. Ezheg. Vses. Paleont. Obshch., 18:24-47. 


\section{PLATES}

Plates 1 to 8 are scanning electron micrographs; Plate 9 to 27 are transmitted-light micrographs. The magnifications are standardized $\times 200$ where possible to allow visual comparisons. Plates 1 and 2 (cryptothoracic and cryptocephalic Nassellaria) are magnified $\times 350$, and some large Spumellaria $\times 100$; these are marked with an asterisk.

Usually there are two illustrations of each specimen, one focused on the silhouette, the other on the surface of the test.

In the explanations of the figures, the sample numbers and slide designations indicate preparations in my collection at the Institut de Géologie, Strasbourg (or, in the case of type specimens, preparations to be deposited in the Département des Sciences de la Terre, 15-43 Bd. du 11 novembre, 69621 Villeurbanne, France), and designations in the form "E.F. M 27/3" indicate England finder positions of the illustrated specimens on the slides. 


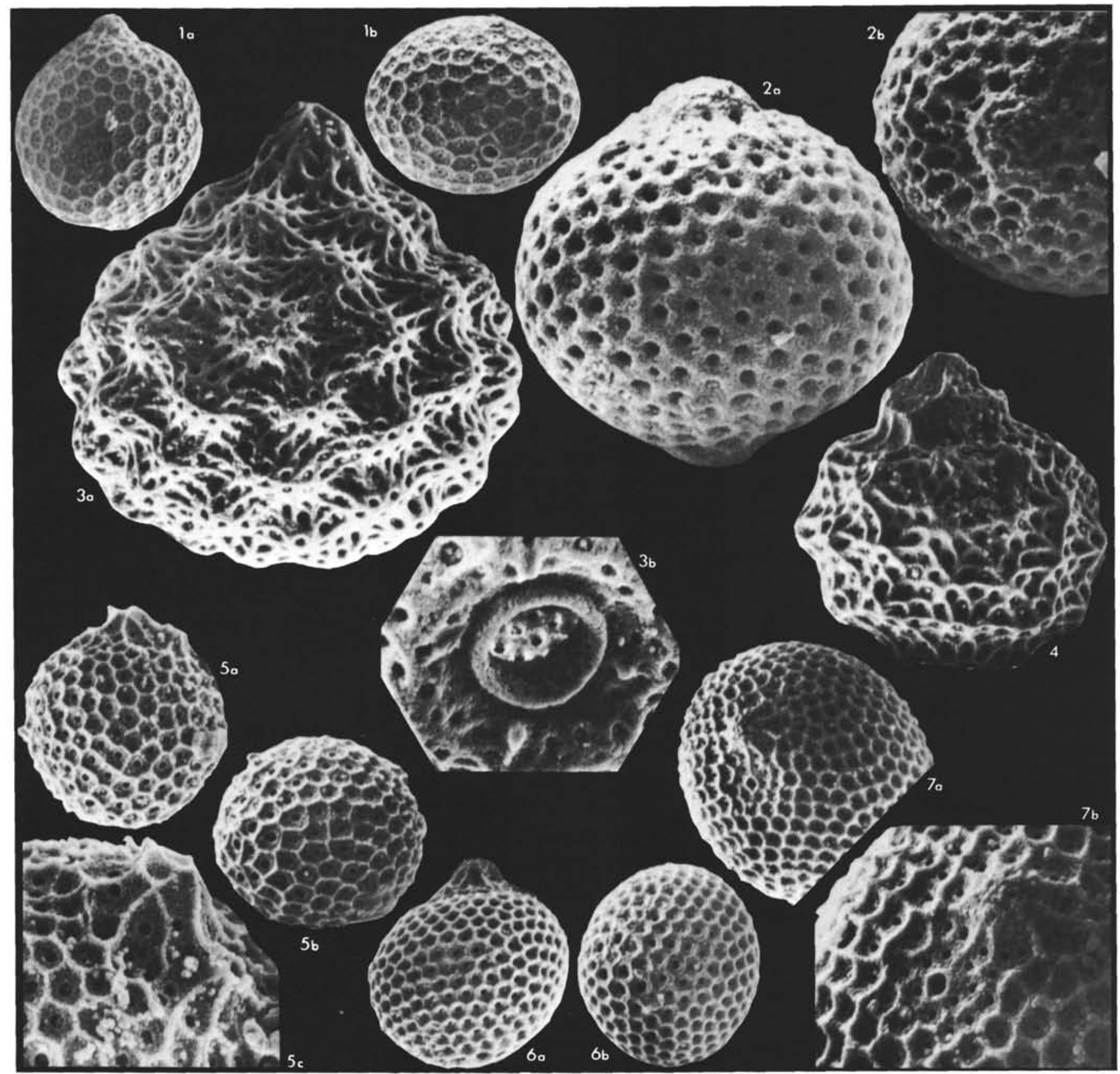

Plate 1. Photomicrographs.

Figures 1a, b. Gongylothorax verbeeki (Tan Sin Hok) $(\times 350)$. Fig. 1a. Lateral view. Fig. 1b. Antapical view showing strongly constricted aperture. Sample 463-90,CC; SEM 781117 and 781118.

Figures 2a, b. Williriedellum carpathicum Dumitricà $(\times 350)$. Fig. 2 a. Lateral view. Fig. 2b. Apical view, showing the complex sutural pore. Sample 463-89-1, 94-95 cm; SEM 781131 and 781132 .

Figures $3 \mathrm{a}, \mathrm{b}$. Williriedellum peterschmittae, n. sp. Fig. 3a. Lateral view $(\times 350)$. Fig. 3b. Antapical view showing the aperture $(\times 700)$. Paratype. Sample 463-90,CC; SEM 781055 and 781056.
Figure 4. Hemicryptocapsa sp. cf . H. prepolyhedra Dumitrică ( $\times 350)$. Sample 463-89-1, 105-106 cm; SEM 781074.

Figures 5a-c. Cryptamphorella dumitricai, n. sp. Fig. 5a. Lateral view, showing the small circular sutural pore always prominent $(\times 350)$. Fig. 5b. Antapical view showing abdomen without aperture $(\times 350)$. Fig. 5c. Details of cephalis and sutural pore $(\times 700)$. Paratype. Sample 463-89-1, 94-95 cm; SEM 781126 to 781128 .

Figures 6a, b. Cryptamphorella conara (Foreman) $(\times 350)$. Fig. 6a. Lateral view. Fig. 6 b. Antapical view showing the absence of aperture. Sample 463-89-1, 94-95 cm; SEM 781142 and 781143.

Figures 7a, b. Cryptothoracic Nassellaria, gen. and sp. indet. $(\times 350$ and $\times 700$ ). Sample 463-90,CC; SEM 781114 and 781115 . 


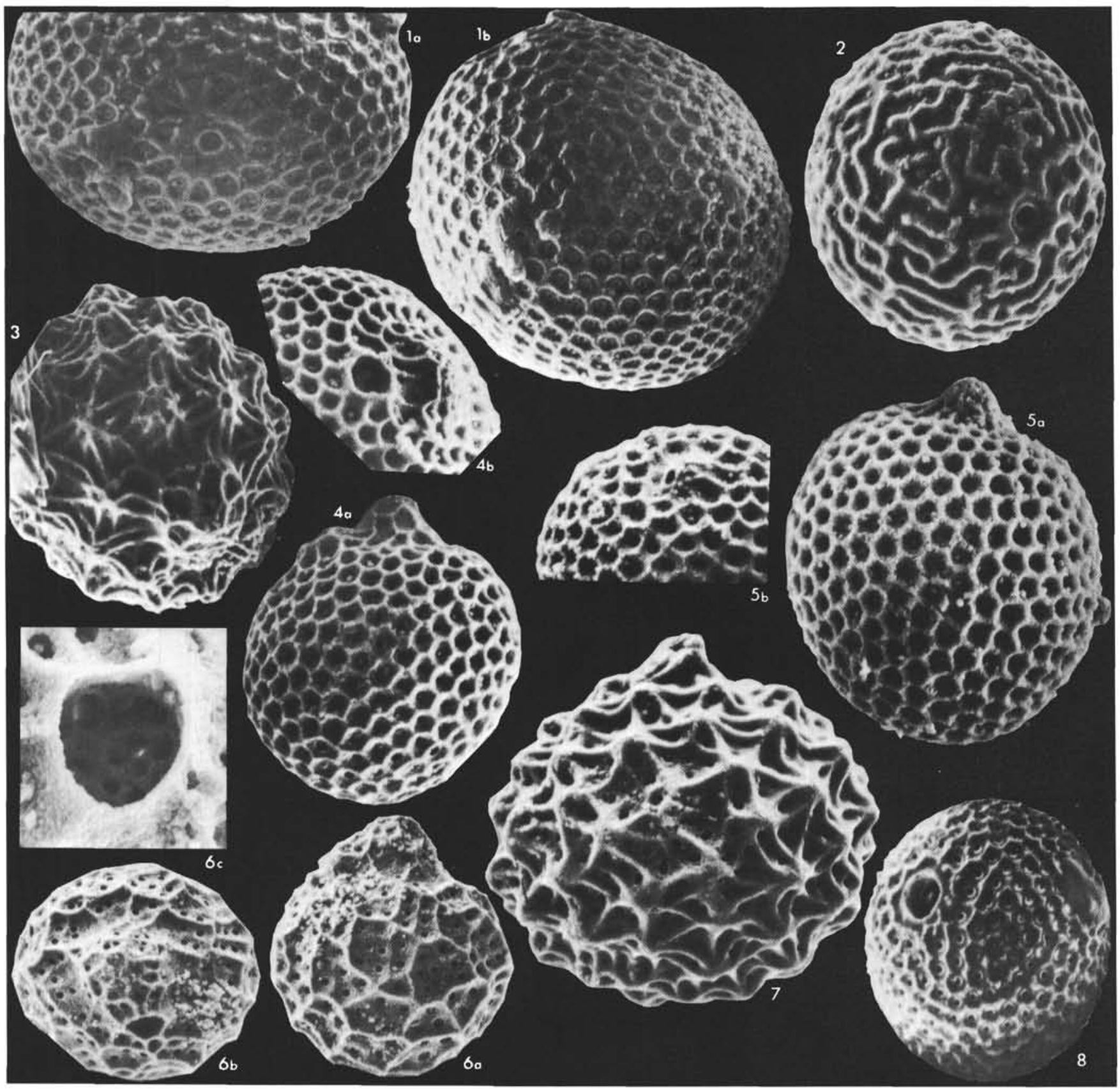

Plate 2. Photomicrographs.

Figures 1a, b. Holocryptocanium barbui Dumitrică $(\times 350)$. Fig. 1a. Antapical view, showing the aperture. Fig. 1b. Lateral view, showing completely encased cephalo-thorax. Sample 466-34-2, 16-17 $\mathrm{cm}$; SEM 781167 and 781168 .

Figure 2. Cryptocephalic Nasselaria gen. and sp. indet. $(\times 350)$. Sample 463-89-1, 94-95 cm; SEM 79057.

Figure 3. Cryptothoracic Nassellaria gen. and sp. indet. $(\times 350)$. Sample 463-89-1, 105-106 cm; SEM 79231.

Figures $4 \mathrm{a}$, b. Cryptamphorella sp. indet. $(\times 350)$ Fig. 4a. Lateral view, showing the prominent sutural pore. Fig. 4b. Detail of sutural pore. Sample 463-89-1, 94-95 cm; SEM 781149 and 781151.
Figures 5a, b. Hemicryptocapsa pseudopilula Tan Sin Hok $(\times 350)$. Fig. 5a. Lateral view. Fig. 5b. Antapical view, showing the strongly constricted aperture. Sample 466-34-2, 16-17 cm; SEM 79371 and 79372 .

Figures 6a-c. Williriedellum gilkeyi Dumitrică. Fig. 6a. Lateral view $(\times 350)$. Fig. 6b. Apical view $(\times 350)$. Fig. 6c. Detail of sutural pore $(\times 1500)$. Sample 463-89-1, 94-95 cm; SEM 781136, 781138, and 781139 .

Figure 7. Cryptocephalic Nassellaria gen. and sp. indet. $(\times 350)$. Sample 463-89-1, 94-95 cm; SEM 79051.

Figure 8. Holocryptocanium sp. (×350). Sample 463-89-1, 105-106 cm; SEM 79261. 


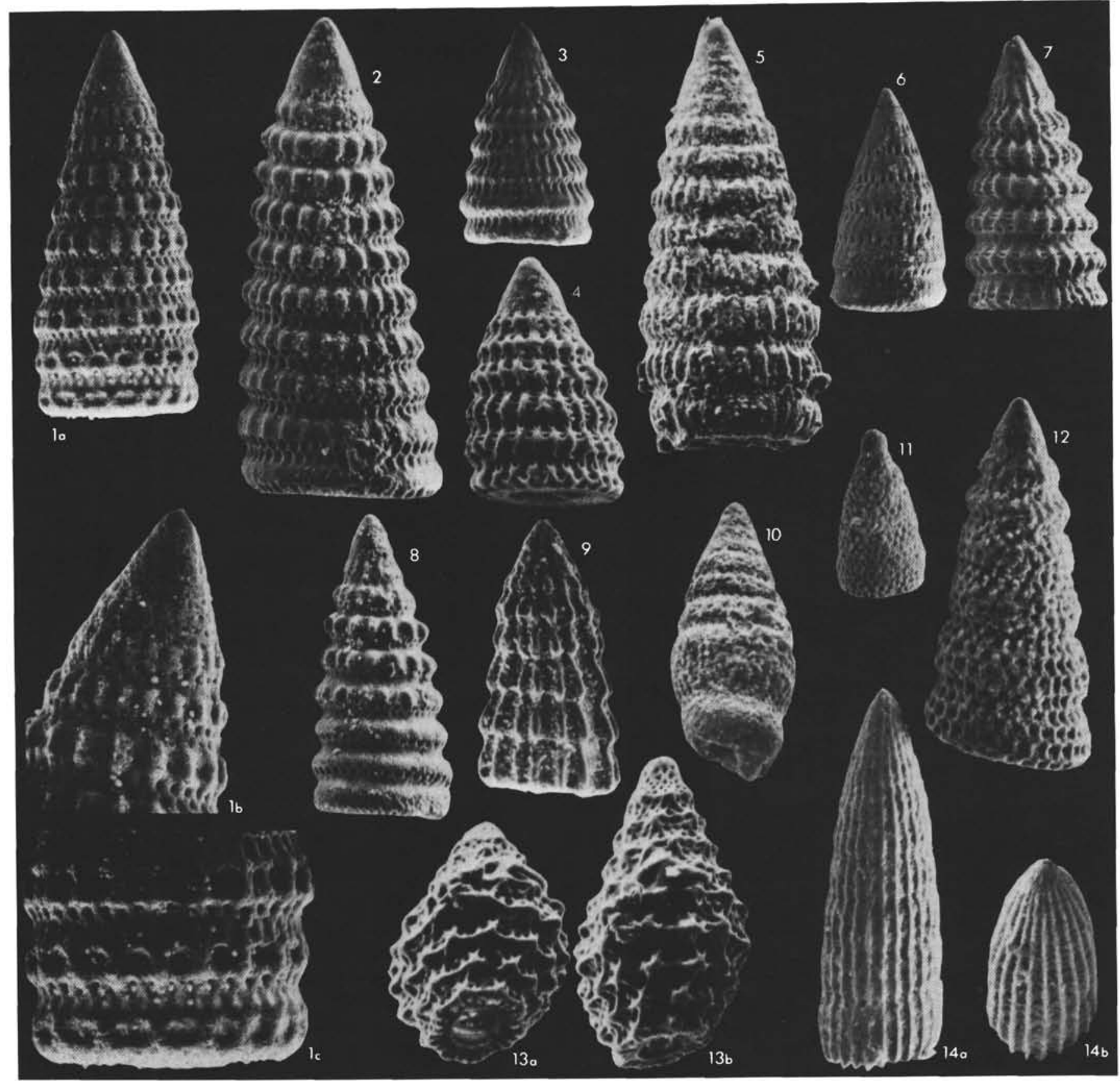

Plate 3. Photomicrographs.

Figures 1a-c. Pseudodictyomitra carpatica (Lozyniak). Fig. 1a. Lateral view $(\times 200)$. Fig. 1b. Cephalis texture, showing molds of nannoplankton $(\times 350)$. Fig. 1c. The three last segments, showing the disposition of the costae and pores $(\times 350)$. Sample 466-34-2, 16-17 cm; SEM 79362 to 79364.

Figure 2. Pseudodictyomitra carpatica (Lozyniak) $(\times 200)$. Sample 463-89-1, 105-106 cm; SEM 781075.

Figure 3. Pseudodictyomitra leptoconica (Foreman) $(\times 200)$. Sample 463-90, CC, SEM 781120.

Figure 4. Parvicingula hsui Pessagno ( $\times 200)$. Sample 463-89-1, 105$106 \mathrm{~cm}$; SEM 79036.

Figure 5. Pseudodictyomitra lodogaensis Pessagno $(\times 200)$. Sample 465A-29-1, 43-44 cm; SEM 79306.

Figure 6. Archaeodictyomitra nuda, n. sp. $(\times 200)$. Paratype. Sample 463-90,CC; SEM 781098.
Figure 7. Archaeodictyomitra puga, n. sp. $(\times 200)$. Paratype. Sample 463-89-1, 94-95 cm; SEM 781154.

Figure 8. Pseudodictyomitra lilyae (Tan Sin Hok) $(\times 200)$. Sample 463-89-1, 94-95 cm; SEM 781134.

Figure 9. Pseudodictyomitra formosa (Squinabol) $(\times 200)$. Sample 466-34-2, 16-17 cm; SEM 79313.

Figure 10. Pseudodictyomitra vestalensis Pessagno $(\times 200)$. Sample 465A-29-1, 43-44 cm; SEM 79323.

Figure 11. Amphipyndax mediocris (Tan Sin Hok) $(\times 200)$. Sample 465A-29-1, 43-44 cm; SEM 79330.

Figure 12. Parvicingula? tekschaensis (Aliev) $(\times 200)$. Sample 466-342, 16-17 cm; SEM 79337.

Figure 13a, b. Parvicingula boesii (Parona) $(\times 200)$. Sample 463-89-1, 94-95 cm; SEM 79064 and 79065.

Figures 14a, b. Archaeodictyomitra sp. $(\times 200)$. Sample 466-34-2, 16-17 cm; SEM 79345 and 79366. 


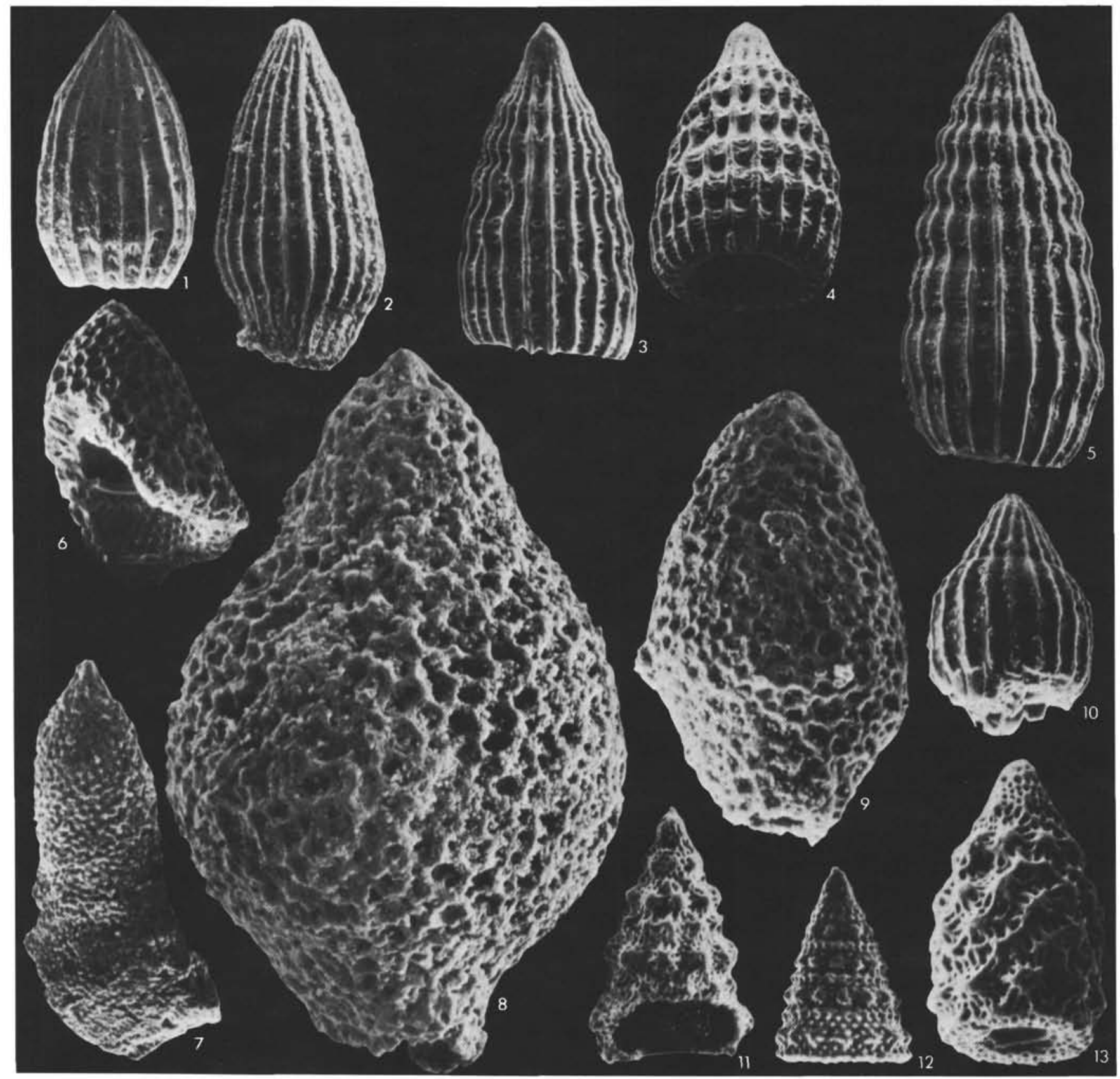

Plate 4. Photomicrographs.

Figure 1. Archaeodictyomitra $\mathrm{sp} .(\times 200)$. This species, very similar to $A$. vulgaris, has two rows of pores at each segment. Sample 463-90,CC; SEM 781109.

Figure 2. Archaeodictyomitra vulgaris Pessagno $(\times 200)$. Sample 463-89-1, 105-106 cm; SEM 781079.

Figure 3. Archaeodictyomitra sp. cf. A. puga n. sp. $(\times 200)$. Sample 463-89-1, 105-106 cm; SEM 781067.

Figure 4. Archaeodictyomitra sp. cf. A. puga, n. sp. $(\times 200)$. This specimen has a less pronouncedly undulating outline than typical A. puga (see PI. 3, Fig. 7). Sample 463-89-1, 105-106 cm; SEM 781068.

Figure 5. Archaeodictyomitra pseudoscalaris (Tan Sin Hok) $(\times 200)$. Sample 463-89-1, 105-106 cm; SEM 781076.

Figure 6. Obesacapsula somphedia (Foreman) $(\times 200)$. Details of cephalis and intersegmental structures. Sample 465A-29-1, 43-44 cm; SEM 79332.
Figure 7. Obesacapsula somphedia (Foreman) $(\times 200)$. Atypical specimen, less inflated.Sample 465A-29-1, 43-44 cm; SEM 79310.

Figure 8. Obesacapsula somphedia (Foreman) $(\times 200)$. Large, typical morphotype. Sample 465A-29-1, 43-44 cm; SEM 79308.

Figure 9. Obesacapsula somphedia $($ Foreman $)(\times 200)$. Small morphotype. Sample 465A-29-1, 43-44 cm; SEM 79294.

Figure 10. Thanarla pulchra (Squinabol) $(\times 200)$. Sample 463-89-1, 94-95 cm; SEM 781178.

Figure 11. Xitus spicularius (Aliev) $(\times 200)$. Note the small broken spine and the great number of rows pores. Sample 463-89-1, 105$106 \mathrm{~cm}$; SEM 79280.

Figure 12. Xitus sp. cf. X. spicularius (Aliev) $(\times 200)$. Sample 463 89-1, 105-106 cm; SEM 79250.

Figure 13. Parvicingula sp. cf. P. boesii (Parona) $(\times 200)$. The shape of the last segments from this morphotype is more cylindrical than the shape of the original species (see Pl. 3, Figs. 13a, b). Sample 463-89-1, 94-95 cm; SEM 79061. 


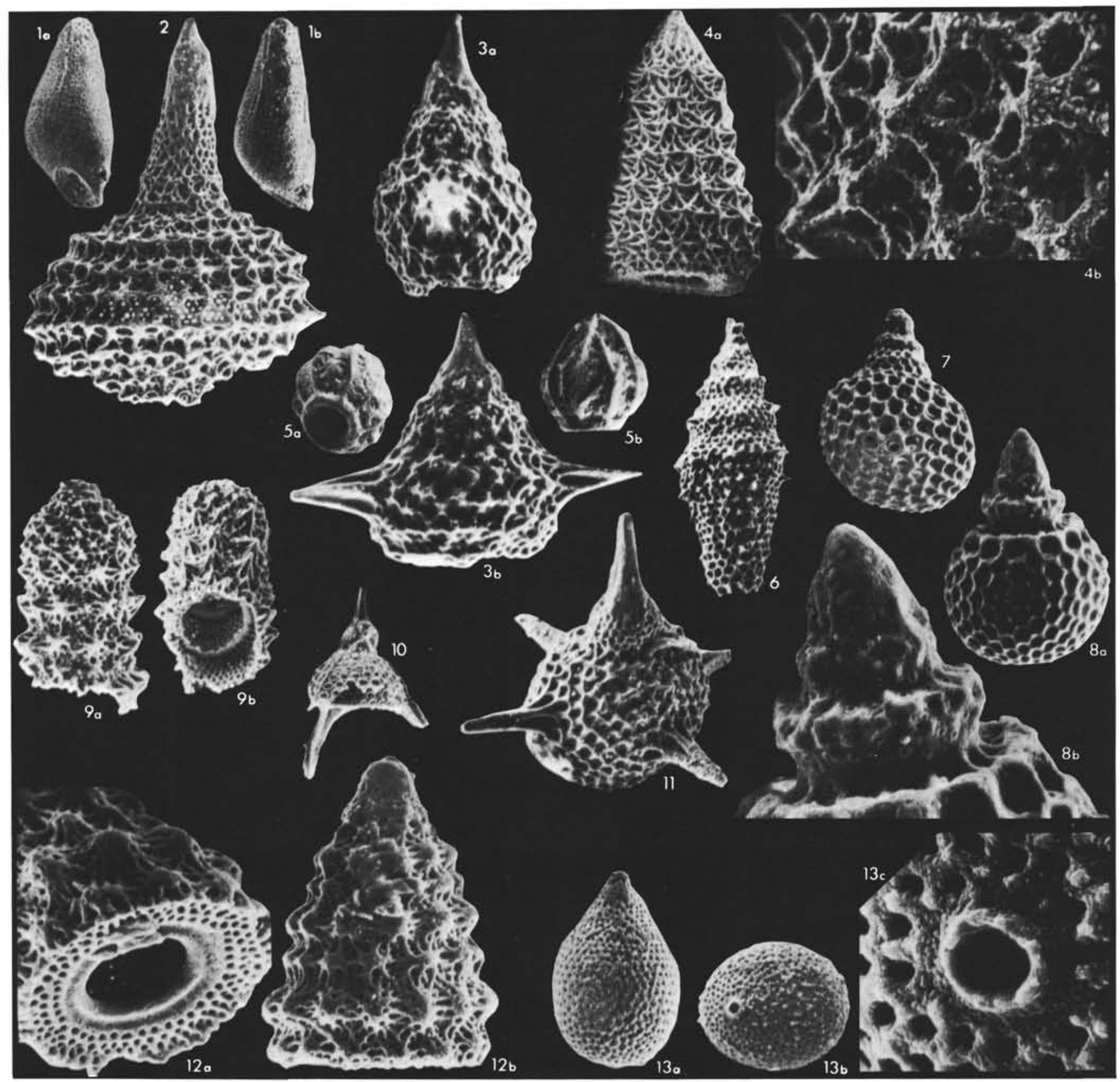

Plate 5. Photomicrographs.

Figures 1a, b. Eucyrtis columbaria Renz $(\times 200)$. Sample 463-90,CC; SEM 781008 and 781009.

Figure 2. Lithocampe chenodes Renz $(\times 200)$. Note the five rows of pores and the relative little size of the specimen. Sample 463-90,CC; SEM 781004.

Figures 3a, b. Dibolachras tytthopora Foreman $(\times 200)$. Sample 46389-1, 94-95 cm; SEM 781174 and 781175.

Figures 4 a, b. Xitus alievi (Foreman). Fig. 4a. Lateral view $(\times 200)$. Fig. 4b. Details of the wall structure $(\times 750)$. Sample 463-90,CC; SEM 78988 and 78990.

Figures 5a, b. Gen. and sp. indet. (×200). Sample 465A-29-1, 43-44 cm; SEM 79327 and 79328.

Figure 6. Eucyrtis elido, n. sp. $(\times 200)$. Sample 463-90,CC; SEM 781002.

Figure 7. Siphocampium? davidi, n. sp. $(\times 200)$. Sample 463-90,CC; SEM 781010.
Figures 8 a, b. Sethocapsa uterculus (Parona) $(\times 200)$. Fig. 8a. Lateral view. Fig. 8 b. Details of cephalis $(\times 575)$. Sample 463-89-1, 105-106 cm; SEM 79228 and 79229.

Figures 9a, b. Xitus sp. A ( $\times 200)$. Sample 463-89-1, 94-95 cm; SEM 781183 and 781184 .

Figure 10. Dictyophimus gracilis Tan Sin Hok $(\times 200)$. Sample 463-90,CC; SEM 781011.

Figure 11. Podobursa triacantha (Fischli) $(\times 200)$. Sample 463-89-1, 105-106 cm; SEM 781066.

Figures 12 a, b. Xitus spicularius (Aliev) $(\times 200)$. Fig. 12a. Detail of antapical part $(\times 350)$. Fig. $12 \mathrm{~b}$. Lateral view $(\times 200)$. Sample 463-89-1, 105-106 cm; SEM 781072 and 781166.

Figures 13a-c. Theocorys renzae, n. sp. $(\times 200)$. Fig. 13a. Lateral view $(\times 200)$. Fig. 13b. Antapical view, showing the constricted aperture $(\times 200)$. Fig. 13c. Aperture $(\times 1500)$. Paratype. Sample 463-89-1, 105-106 cm; SEM 781061, 781062, and 781158. 


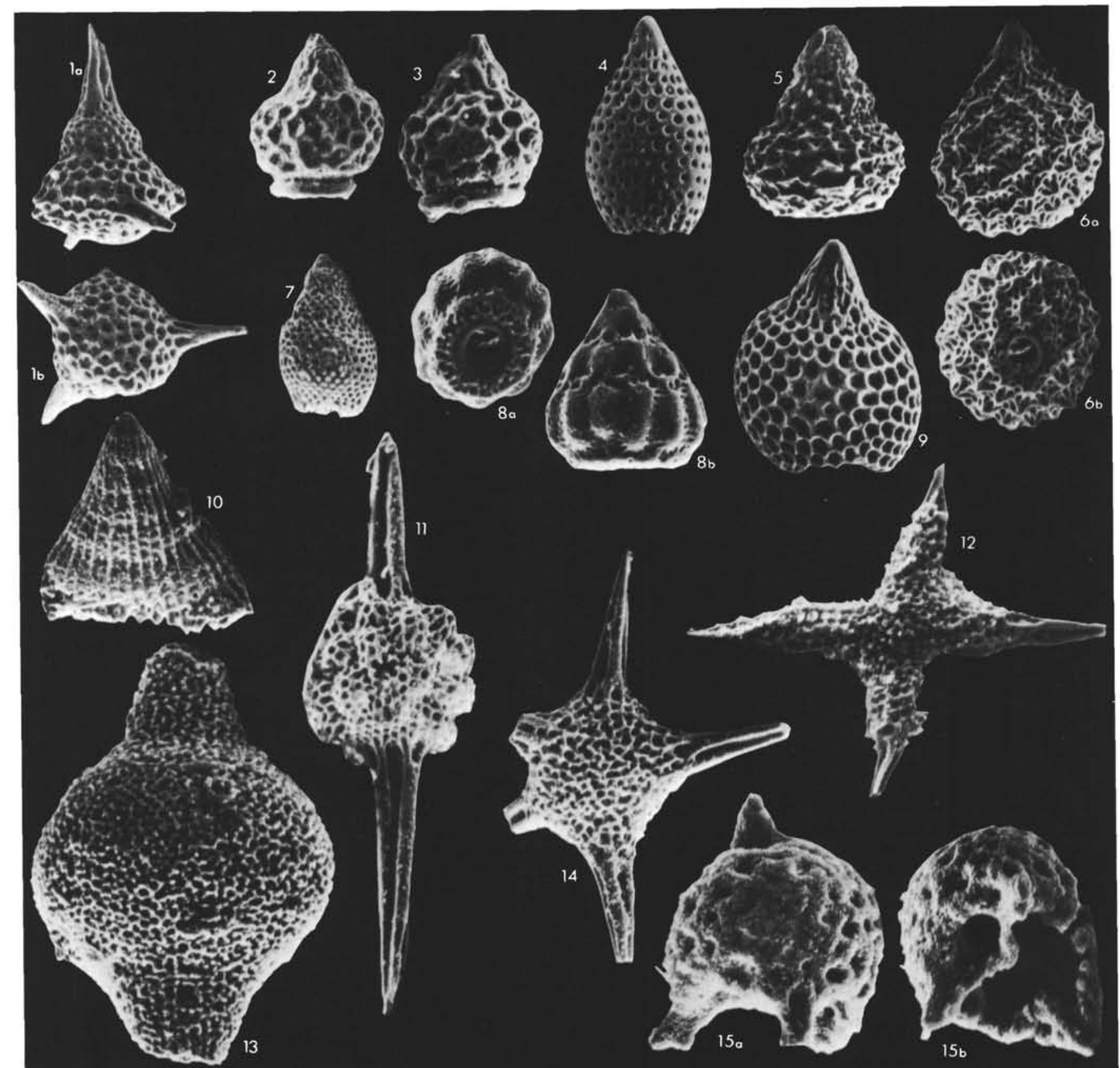

Plate 6. Photomicrographs.

Figures 1a, b. Podobursa tricola Foreman $(\times 200)$. Fig. 1a. Lateral view, showing the small abdomen. Fig. 1b. Antapical view, with broken apertural tube. Sample 463-89-1, 94-95 cm; SEM 781129 and 781130 .

Figure 2. Rhopalosyringium majuroensis, n. sp. $(\times 200)$. Paratype. Sample 465A-29-1, 43-44 cm; SEM 79325.

Figure 3. Rhopalosyringium majuroensis, n. sp. $(\times 200)$. Sample 465A-29-1, 43-44 cm; SEM 79304.

Figure 4. Stichocapsa cribata Hinde $(\times 200)$. Sample 463-89-1, 105$106 \mathrm{~cm}$; SEM 79227.

Figure 5. Stichocapsa rutteni Tan Sin Hok $(\times 200)$. Sample $463-90$, CC; SEM 781103.

Figures 6a, b. Cyrtocapsa grutterinki Tan Sin Hok $(\times 200)$. Fig. 6a. Lateral view. Fig. 6b. Antapical view, showing the aperture. Sample 463-89-1, 105-106 cm; SEM 79269 and 79270.
Figure 7. Gen. and sp. indet. $(\times 200)$. Sample $463-89-1,105-106 \mathrm{~cm}$; SEM 79277.

Figures 8a, b. Gen. and sp. indet. $(\times 200)$. Sample 463-89-1, 105-106 $\mathrm{cm}$; SEM 79241 and 79242.

Figure 9. ?Cyrtocapsa asseni var. $\alpha$ Tan Sin Hok $(\times 200)$. Sample 46389-1, 105-106 cm; SEM 79255.

Figure 10. ?Mita magnifica Pessagno $(\times 200)$. Sample 466-34-2, 16-17 $\mathrm{cm}$; SEM 79355

Figure 11. Acaeniotyle umbilicata (Rüst) $(\times 200)$. Sample 463-89-1, 105-106 cm; SEM 79273.

Figure 12. Higumastra sp. $(\times 200)$. Sample 463-89-1, 105-106 cm; SEM 79033.

Figure 13. ?Spongodruppa cocos Rüst $(\times 200)$. Sample 466-34-2, 16$17 \mathrm{~cm}$; SEM 79380.

Figure 14. Pentasphaera longispina Squinabol $(\times 200)$. Sample 46389-1, 94-95 cm; SEM 781191.

Figures $15 \mathrm{a}$, b. Saitoum cepeki, n. sp. $(\times 700)$. Specimen with small pores. Sample 463-90,CC; SEM 781111 and 781112. 


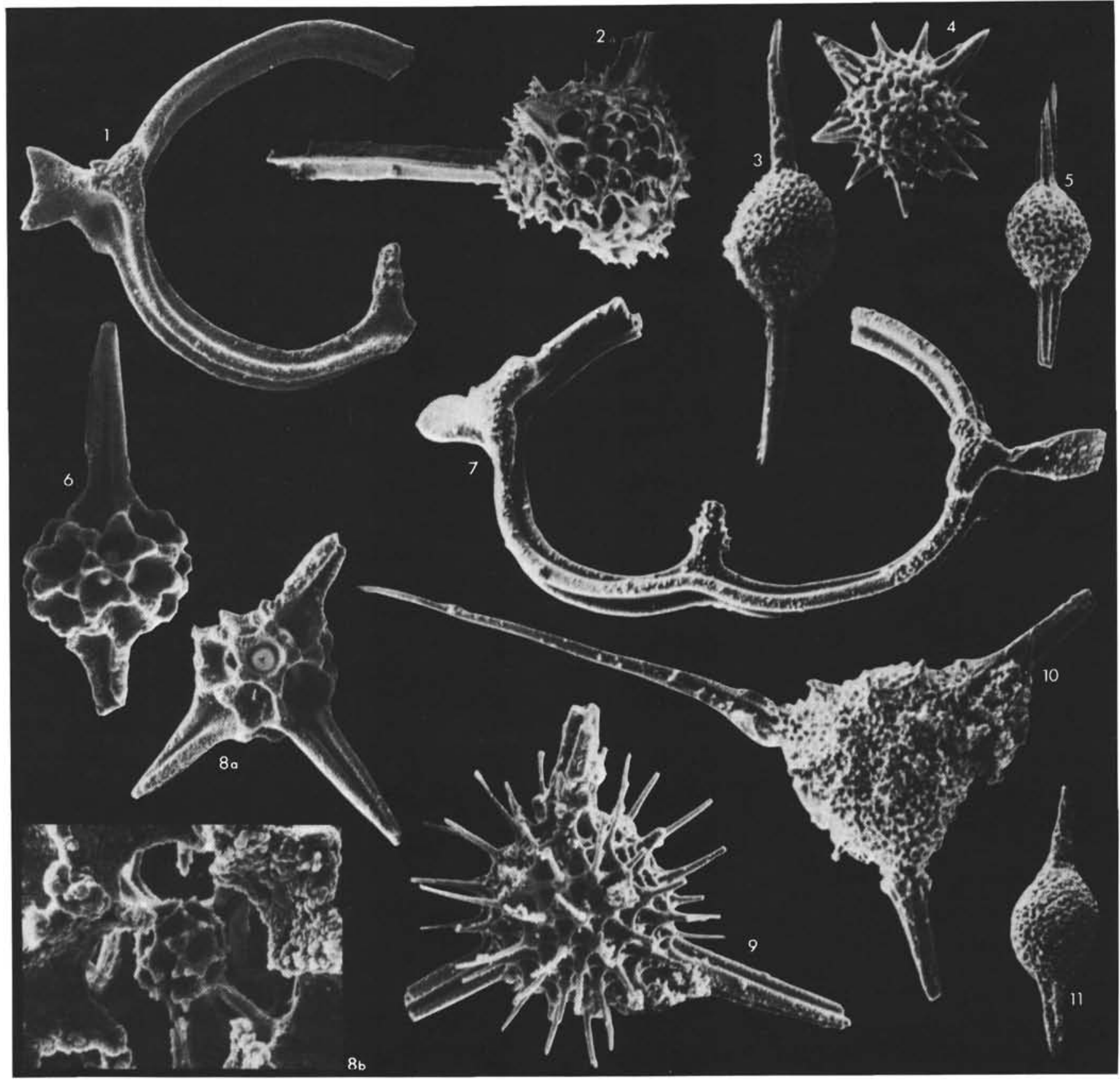

Plate 7. Photomicrographs.

Figure 1. Acanthocircus dicranacanthos (Squinabol) $(\times 200)$. Sample 463-90, CC; SEM 78992.

Figure 2. Hexastylurus magnificus (Squinabol) $(\times 200)$. Sample 46389-1, 105-106 cm; SEM 79044.

Figure 3. Archaeospongoprunum tehamaensis Pessagno $(\times 200)$. Sample 463-89-1, 105-106 cm; SEM 79045.

Figure 4. Gen. and sp. indet. $(\times 200)$. Sample 463-89-1, 94-95 cm; SEM 79072.

Figure 5. Archaeospongoprunum tehamaensis Pessagno $(\times 200)$. Note the pentaradiate and triradiate character of the polar spines. Sample 463-89-1, 105-106 cm; SEM 79278.
Figure 6. Sphaerostylus lanceola (Parona) $(\times 200)$. Sample 463-89-1, 105-106 cm; SEM 79047.

Figure 7. Acanthocircus sp. $(\times 200)$. Sample 463-89-1, 105-106 cm; SEM 79259.

Figures 8 a, b. Staurosphaera septemporata Parona $(\times 200)$. Fig. $8 \mathrm{a}$. Lateral view. Fig. 8 b. Details of the first medullary shell $(\times 700)$. Sample 463-89-1, 105-106 cm; SEM 781077 and 781078.

Figure 9. Alievium helenae, n. sp. $(\times 200)$. Paratype. Sample 46389-1, 105-106 cm; SEM 781064.

Figure 10. Alievium antiguum Pessagno $(\times 200)$. Sample 463-89-1, 94-95 cm; SEM 781169.

Figure 11. Archaeospongoprunum cortinaensis Pessagno $(\times 200)$. Sample 465A-29-1, 43-44 cm; SEM 79341. 


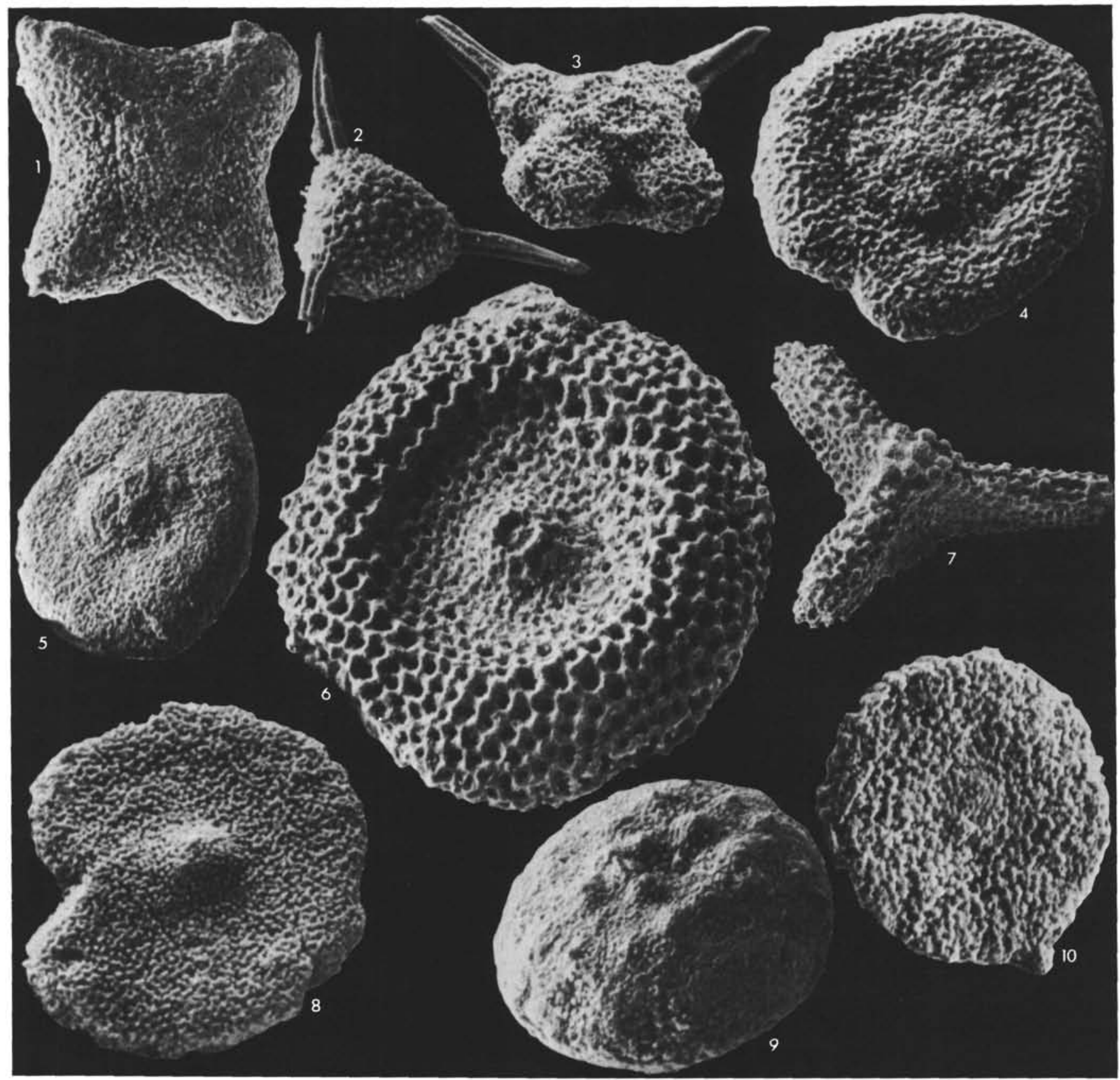

\section{Plate 8. Photomicrographs.}

Figure 1. Histiastrum aster Lipman $(\times 200)$. Sample 465A-29-1, 43-44 cm; SEM 79309.

Figure 2. Alievium antiguum Pessagno $(\times 200)$. Sample 463-89-1, 105-106 cm; SEM 79042.

Figure 3. Crucella sp. cf. C. cachensis Pessagno $(\times 200)$. Sample 46389-1, 94-95 cm; SEM 781181.

Figure 4. ?Spongodiscus renillaeformis Campbell and Clark $(\times 200)$. Sample 465A-29-1, 43-44 cm; SEM 79316.
Figure 5. ?Spongodiscus renillaeformis Campbell and Clark $(\times 200)$. Sample 465A-29-1, 43-44 cm; SEM 79342.

Figure 6. Orbiculiforma chartonae, n. sp. $(\times 200)$. Paratype. Sample 465A-29-1, 43-44 cm; SEM 79339.

Figure 7. Paronaella sp. $(\times 200)$. Sample 463-90,CC; SEM 781012.

Figure 8. ?Spondiscus renillaeformis Campbell and Clark $(\times 200)$. Sample 466-34-2, 16-17 cm; SEM 79373.

Figure 9. Patellula planoconvexa (Pessagno) $(\times 200)$. Sample 465A-29-1, 43-44 cm; SEM 79336.

Figure 10. Spongodiscus americanus Kozlova $(\times 200)$. Sample 465A29-1, 43-44 cm; SEM 79317. 

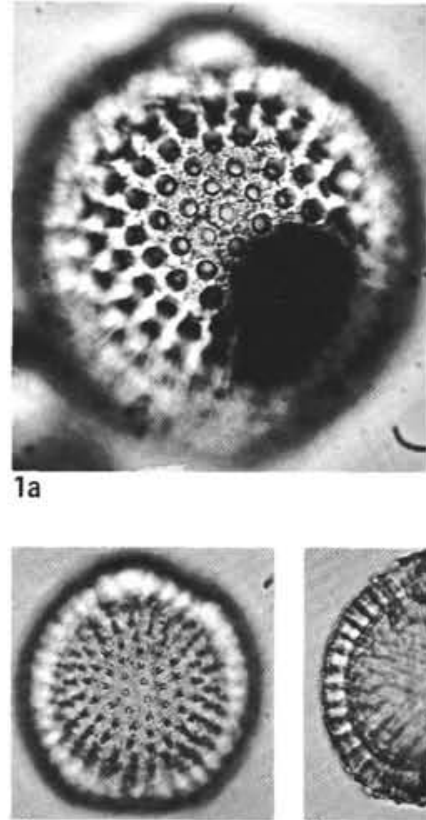

$4 a$

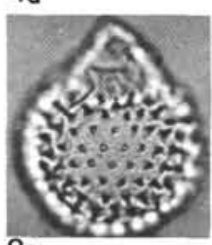

$8 \mathrm{a}$

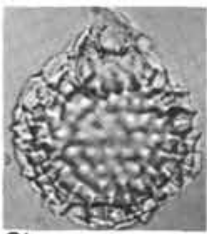

$8 b$

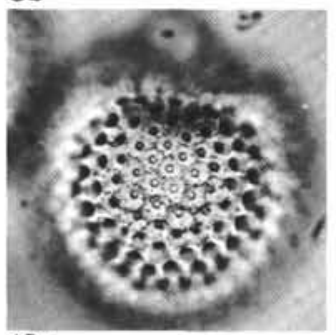

$13 a$

$4 b$

$9 a$

$9 b$

$13 b$

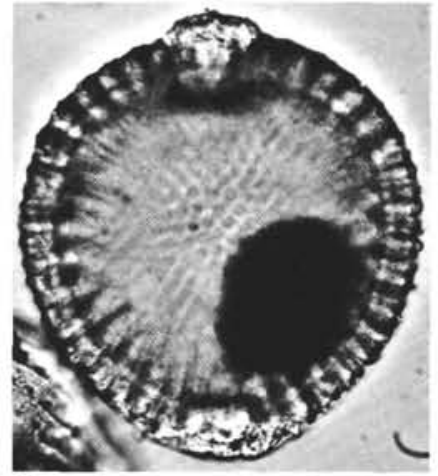

$1 \mathrm{~b}$
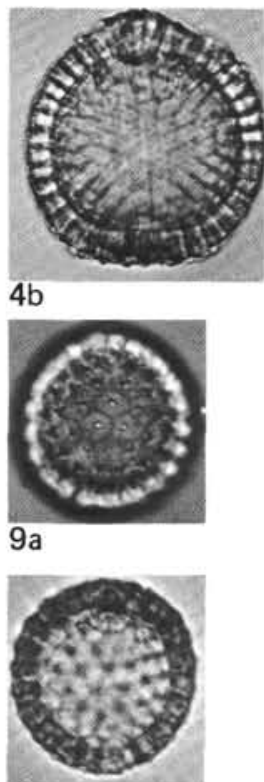

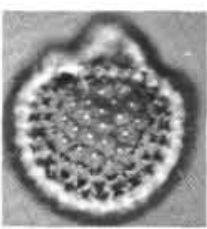

$5 a$

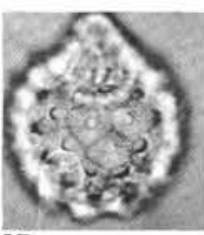

$10 a$

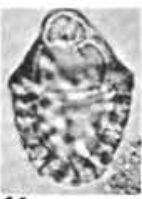

$11 a$
$11 \mathrm{~b}$

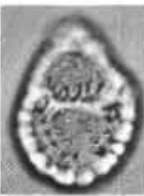

$2 a$

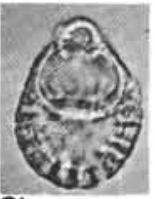

$2 b$

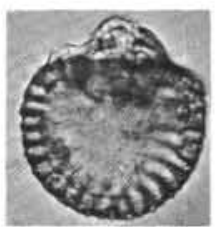

$5 b$

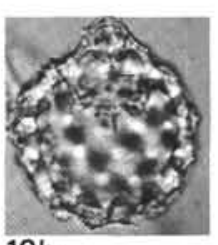

$10 \mathrm{~b}$
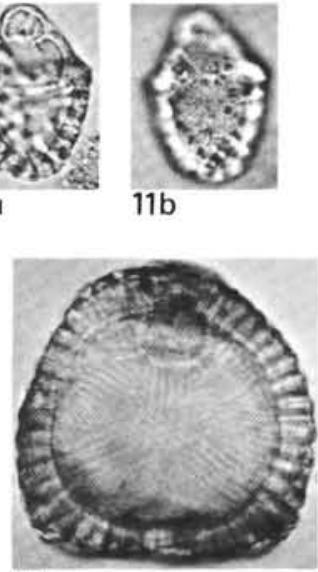

14

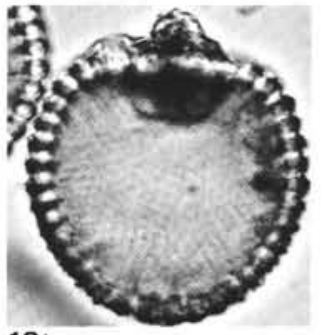

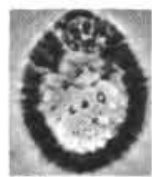

$12 a$

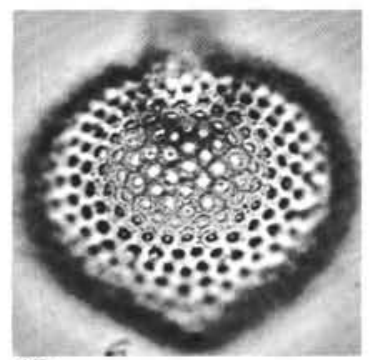

$15 a$

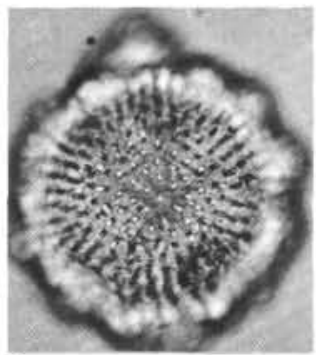

$3 a$

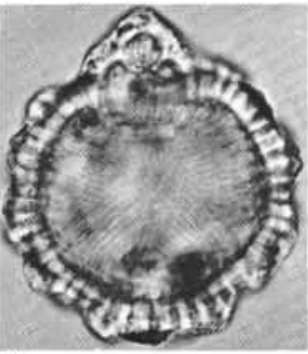

$3 b$
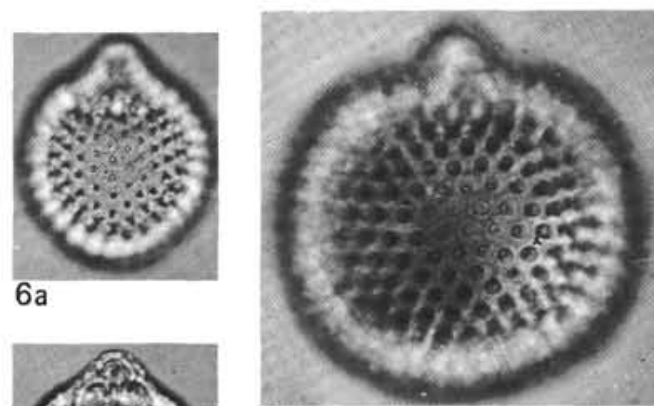

$7 a$

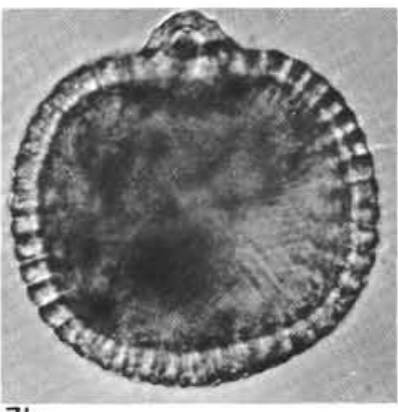

$7 b$

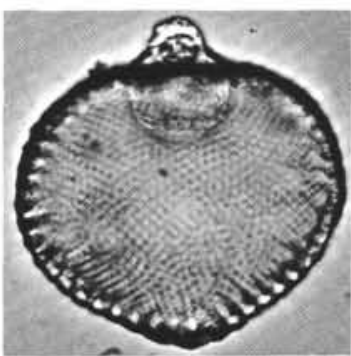

$15 b$
Plate 9. Photomicrographs.

Figures 1a, b. Gongylothorax baumgartneri, n. sp. Holotype. Sample 463-90,CC; slide 2; E.F. T 45/3.

Figures 2a, b. Diacanthocapsa boersmae, n. sp. Holotype. Sample 463-92-1, 0-1 cm; slide 2; E.F. N 44/4.

Figures $3 \mathrm{a}, \mathrm{b}$. Williriedellum peterschmittae, n. sp. Paratype. Sample 463-89-1, 105-106 cm; slide 3; E.F. H 35/2.

Figures 4a, b. Holocryptocapsa hindei Tan Sin Hok. Sample 463-91, CC; slide 2; E.F. F $42 / 1$.

Figures 5a, b. Cryptamphorella dumitricai, n. sp. Sample 463-89-1, 105-106 cm; slide 6; E.F. R 30/2.

Figures $6 \mathrm{a}, \mathrm{b}$. Cryptamphorella challengeri, n. sp. Holotype. Sample 463-88-1, 52-53 cm; slide 5; E.F. X 34/4.

Figures $7 \mathrm{a}, \mathrm{b}$. Hemicryptocapsa? sp. cf. H. cryptodon Dumitrică. Sample 463-89-1, 105-106 cm; slide 2; E.F. H 50/2.
Figures $8 \mathrm{a}, \mathrm{b}$. Hemicryptocapsa $\mathrm{sp}$. cf. $H$. prepolyhedra Dumitrică. Sample 463-86-1, 31-32 cm; slide 3; E.F. T 44/3.

Figures 9a, b. Gongylothorax verbeeki (Tan Sin Hok). Sample 46385-2, 20-21 cm; slide 2; E.F. O 37/1.

Figures $10 \mathrm{a}$, b. Hemicryptocapsa vincentae, n. sp. Holotype. Sample 463-86, CC; slide 3; E.F. R 56/3.

Figures 11a, b. Diacanthocapsa sp. Sample 463-85-1, 142-143 cm; slide 4; E.F. K 45/2.

Figures 12 a, b. Diacanthocapsa boersmae, n. sp. Paratype. Sample 463-88-1, 52-53 cm; slide 5; E.F. X 35/4.

Figures 13 a, b. Cryptamphorella dumitricai, n. sp. Holotype. Sample 463-90,CC; slide 2; E.F. X 43/2.

Figure 14. Holocryptocapsa hindei Tan Sin Hok. Sample 463-86-1, 31-32 cm; slide 4; E.F. W 29/4.

Figures 15a, b. Cryptamphorella conara (Foreman). Sample 463-90, CC; slide 5; E.F. T $37 / 1$. 

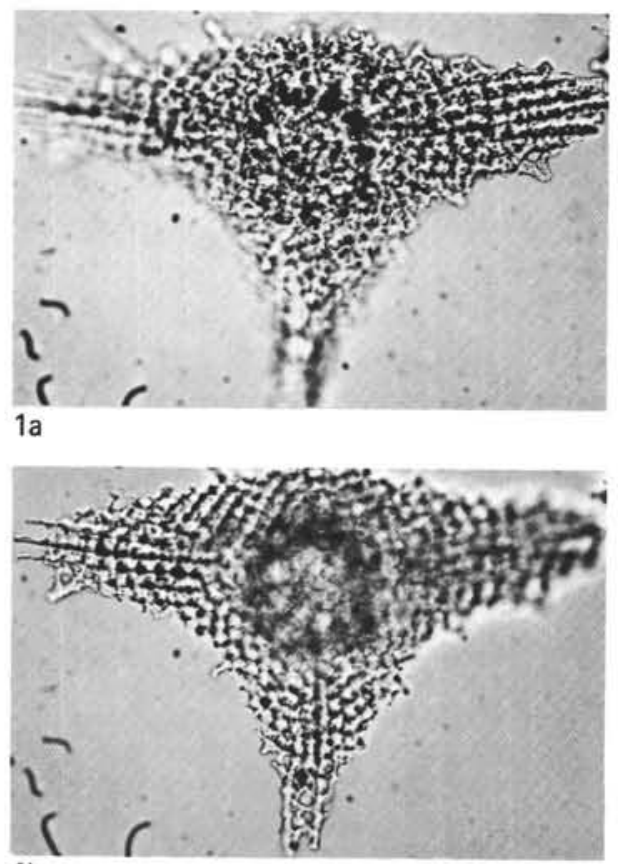

$1 b$

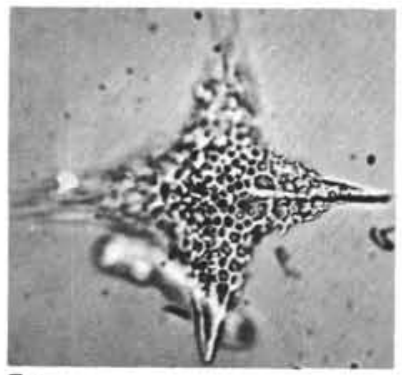

$5 a$

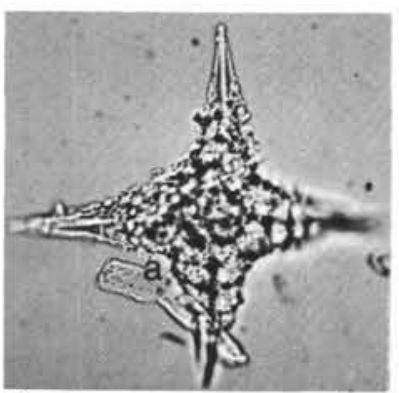

$5 b$

Plate 10. Photomicrographs.

Figures 1a, b. Gen. and sp. indet. Sample 463-90,CC; slide 11; E.F. B $3 / 1$.

Figures 2a, b. Alievium helenae, n. sp. Holotype. Sample 463-90,CC; slide 11; E.F. L 38/3.

Figure 3. Alievium sp. $(\times 100)$. Sample 463-89-1, 94-95 cm; slide 9; E.F. W $32 / 4$.

Figure 4. Amphibrachium? hastatum Renz. Sample 463-87-1, 65-66 $\mathrm{cm}$; slide 3; E.F. Q 44/2.
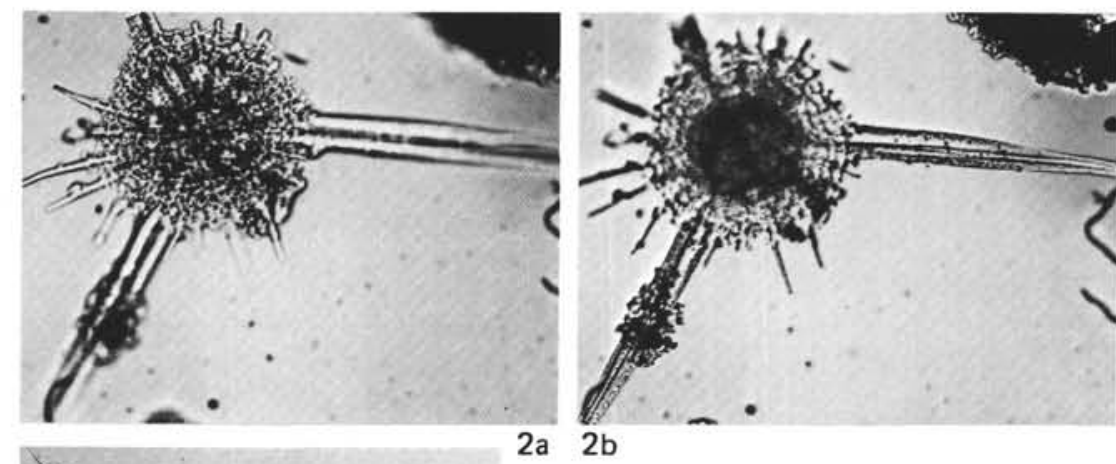

$2 a \quad 2 b$
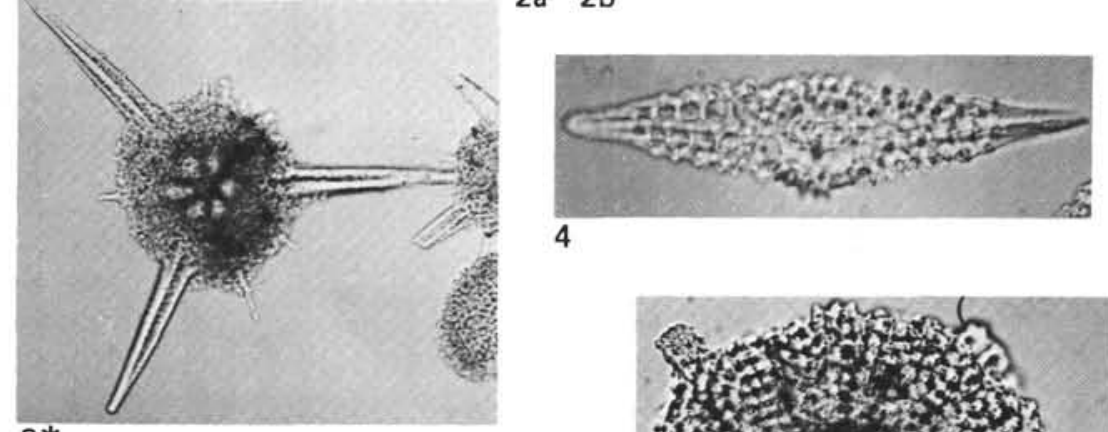

4

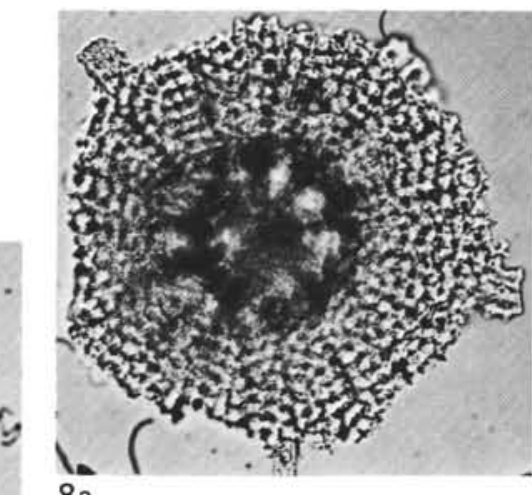

8 a

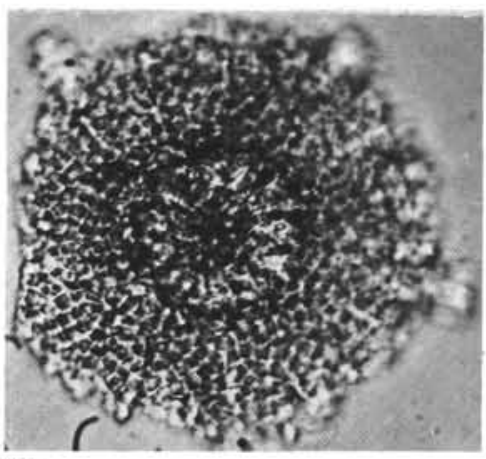

$8 \mathrm{~b}$

Figures 5a, b. Higumastra sp. Sample 463-90,CC; slide 11; E.F. Q $30 / 4$.

Figures 6a, b. Holocryptocanium barbui Dumitrică. Sample 465A29-1, 43-44 cm; slide 17; E.F. U 40/1.

Figures 7a, b. Archaeospongoprunum tehamaensis Pessagno. Sample 463-90,CC; slide 13; E.F. E 39/3.

Figures $8 \mathrm{a}$, b. Gen. and sp. indet. Sample 463-90,CC; slide 14; E.F. U 40/4. 


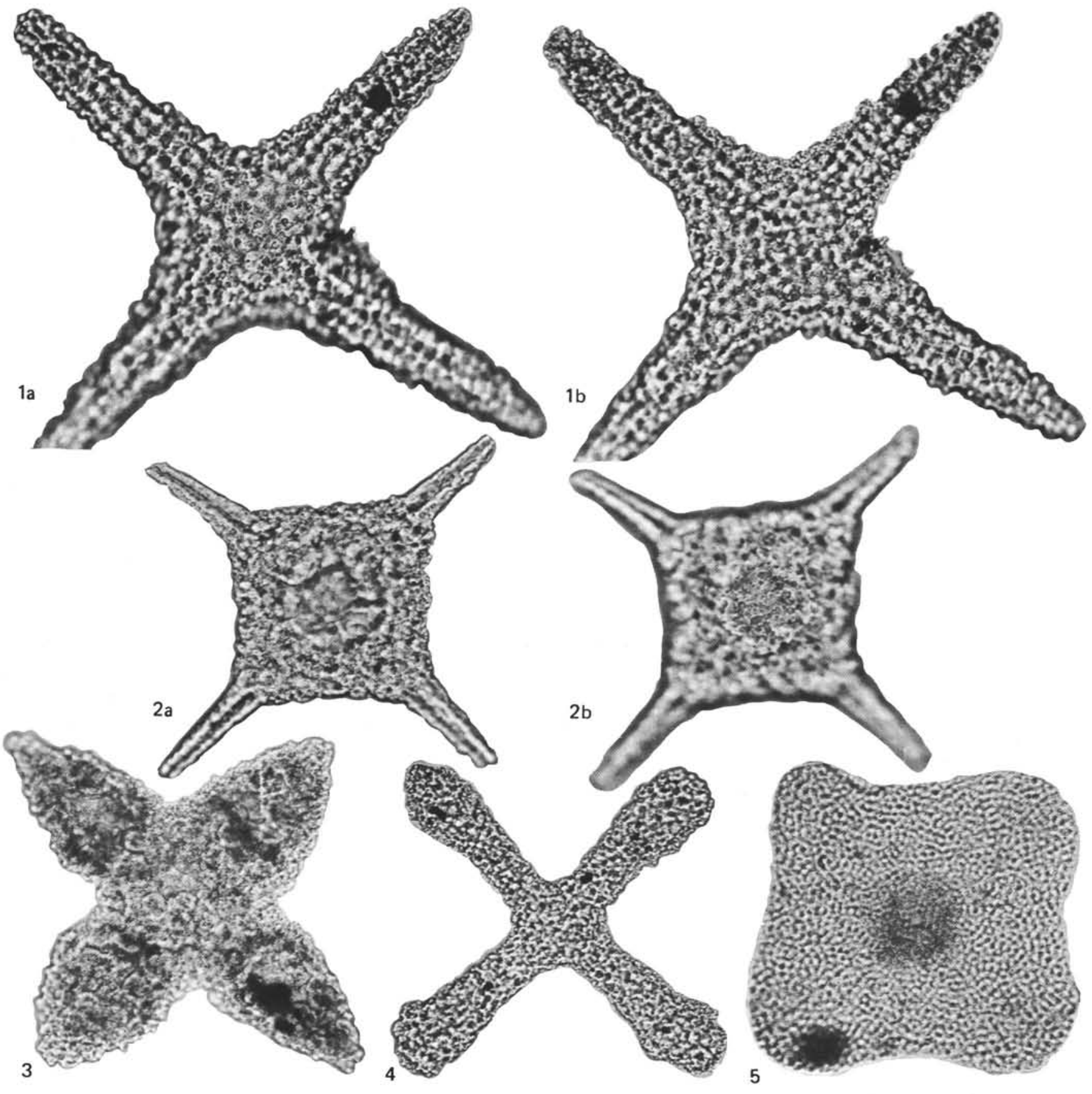

Plate 11. Photomicrographs.

Figures 1a, b. Hagiastrum? euganeum (Squinabol). Sample 463-75-1, 21-22 cm; slide 23; E.F. W 43/1.

Figures 2a, b. Staurocyclia martini Rüst. Sample 463-75-1, 21-22 cm; slide 23 ; E.F. G 50/3.
Figure 3. Crucella $\mathrm{sp}$. indet. Sample 463-66-2, 27-28 cm; slide 4; E.F. U $54 / 1$.

Figure 4. Hagiastrum? subacutum Rüst. Sample 463-75-1, 21-22 cm; slide 21 ; E.F. K 53/1.

Figure 5. Histiastrum aster Lipman. Sample 466-34-2, 16-17 cm; slide 13; E.F. S $46 / 3$. 


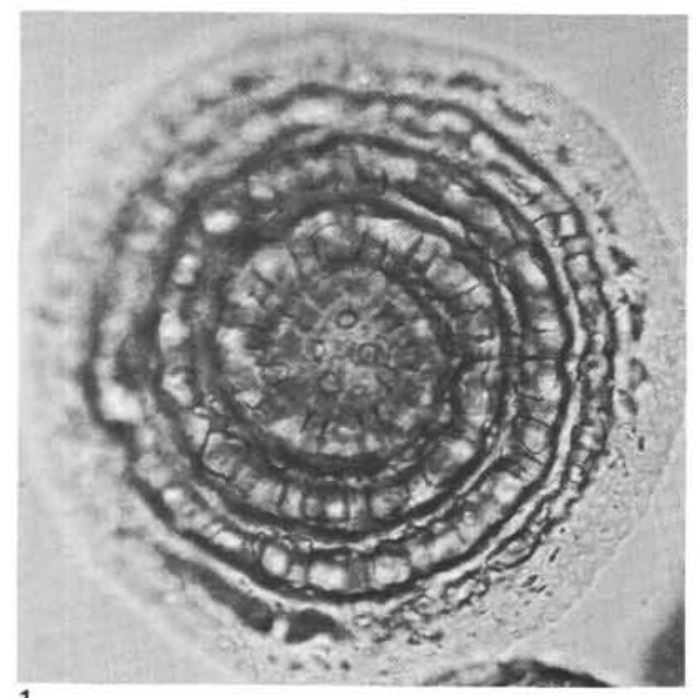

1

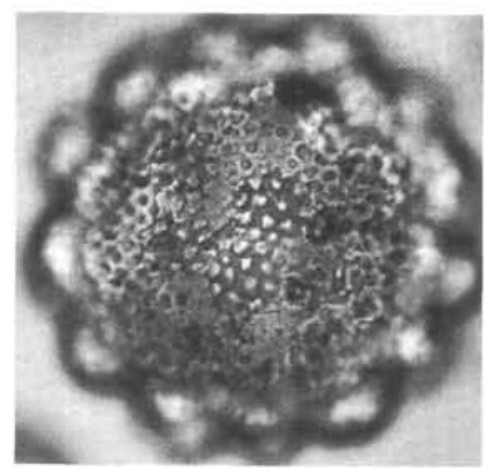

$5 a$

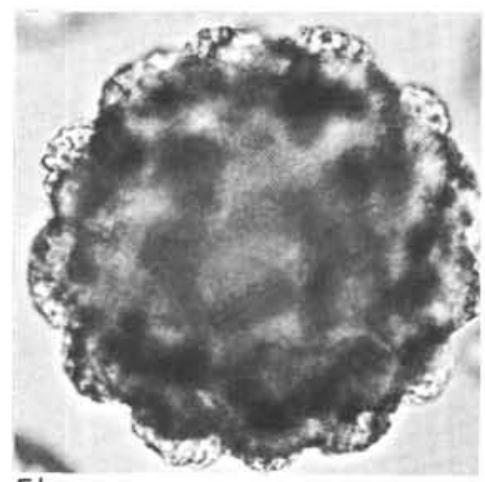

$5 \mathrm{~b}$

Plate 12. Photomicrographs.

Figure 1. Stylochlamyum? sp. group. Sample 463-72-5, 20-21 cm; slide 2; E.F. M 44/4.

Figure 2. Hexalonche? sp. group. Sample 463-72,CC; slide 3; E.F. P $37 / 2$.

Figure 3. Spongobrachium? sp. Sample 463-73-1, 18-19 cm; slide 3; E.F. J $49 / 1$.

Figures 4a, b. Hexalonche? sp. group. Sample 463-76,CC; slide 4; E.F.S $46 / 3$.

2

3

7
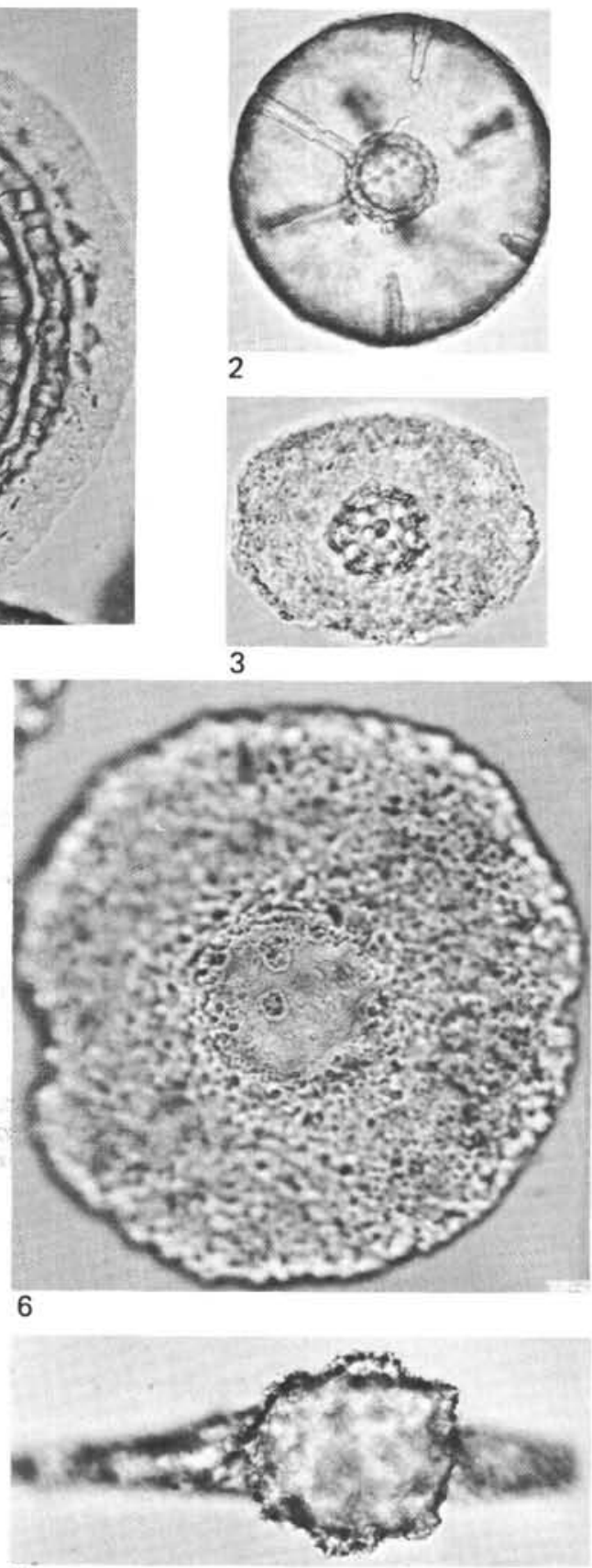

Figures 5a, b. Conosphaera tuberosa Tan Sin Hok. Sample 46390,CC; slide 2; E.F. Q 29/1.

Figure 6. Pseudoaulophacus? excavatus (Rüst). Sample 463-84-1, 5-6 $\mathrm{cm}$; slide 3; E.F. J 30/1.

Figure 7. Triactoma hybum Foreman. Sample 463-73-1, 18-19 cm; slide 3; E.F. W 53/1.

Figure 8. Spongocyclia trachodes Renz. Sample 463-82,CC; slide 7; E.F. K 44/2.

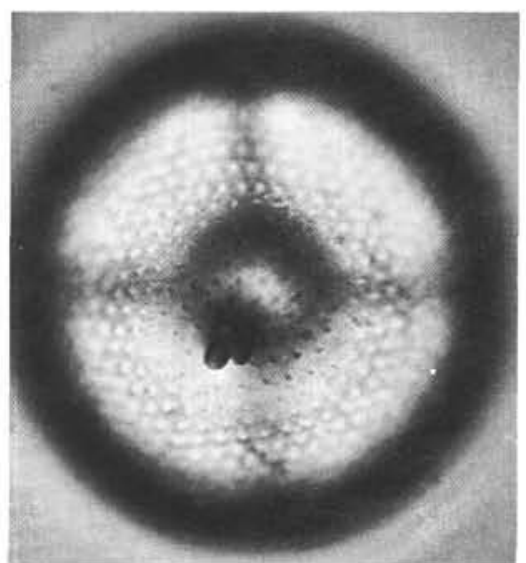

$4 a$

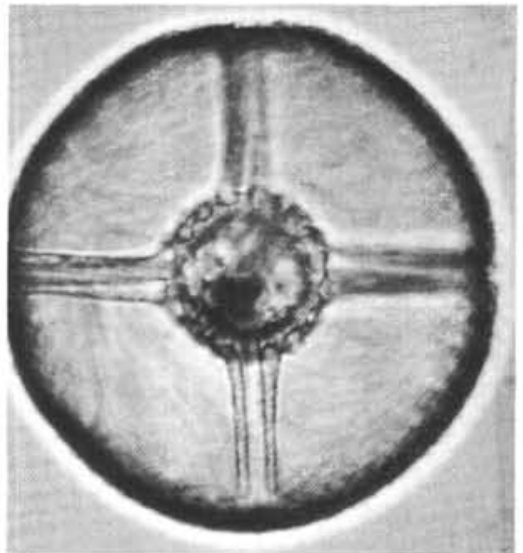

$4 b$

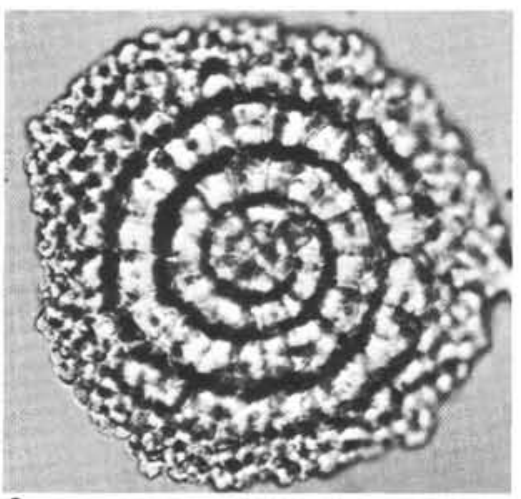




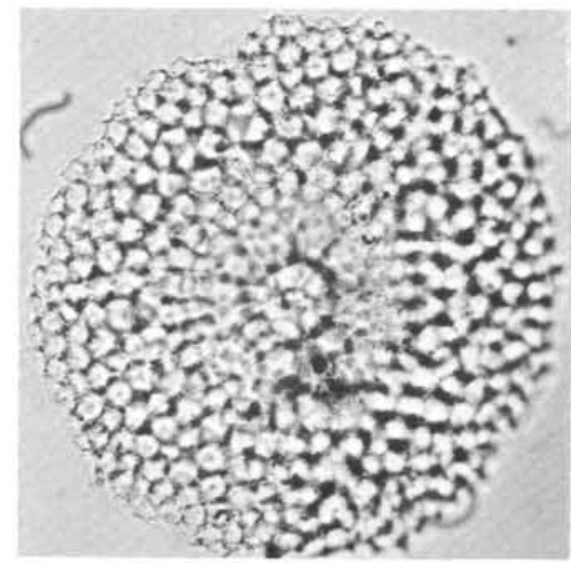

1

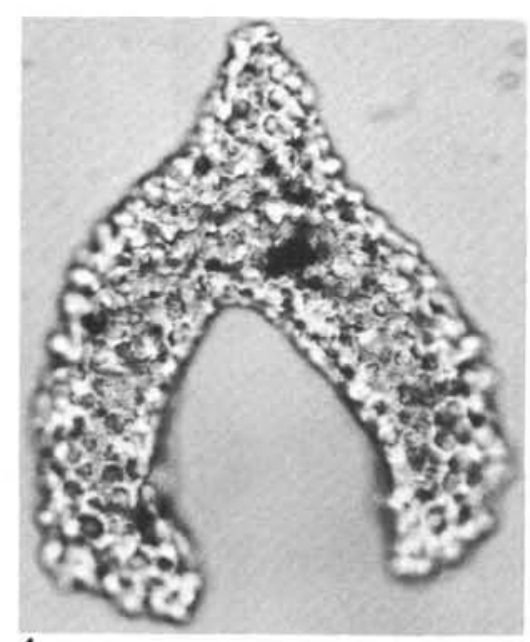

4

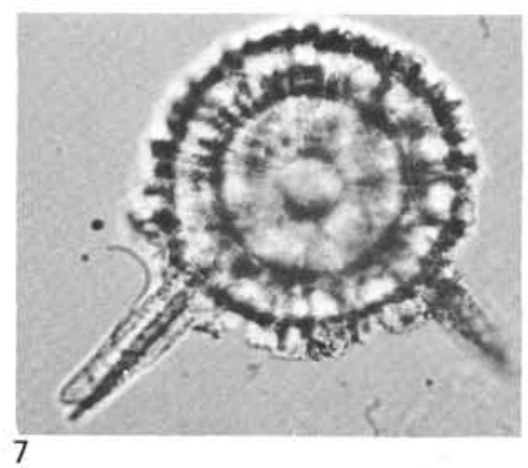

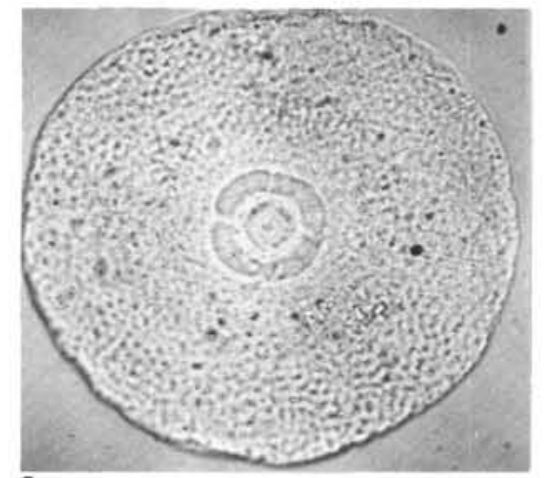

2
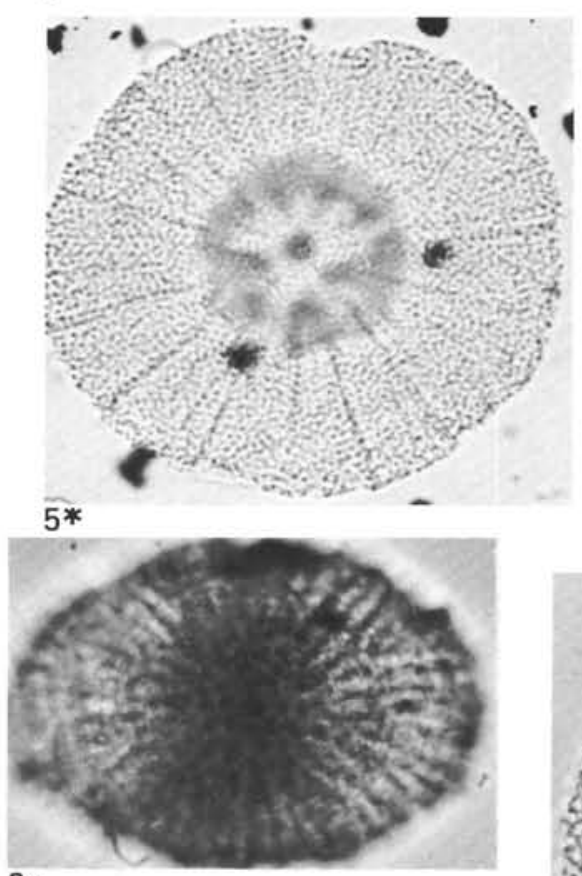

$8 a$

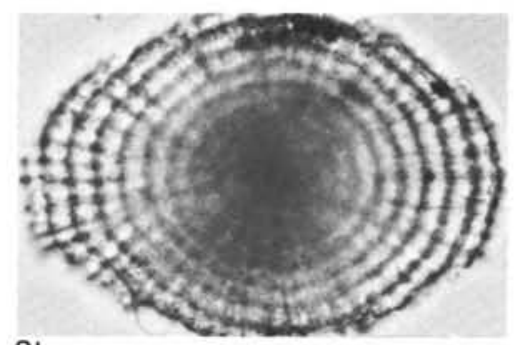

9
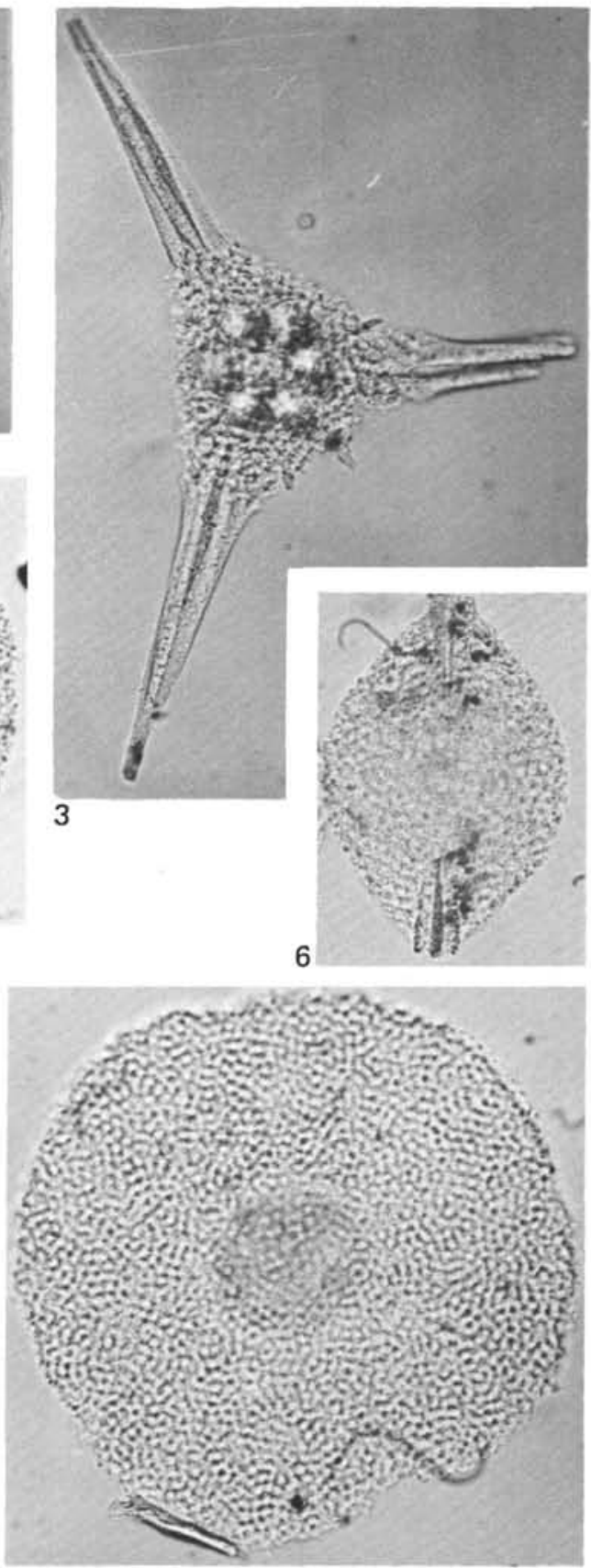

$8 b$

Plate 13. Photomicrographs.

Figure 1. Orbiculiforma chartonae, n. sp. Paratype. Sample 466-34-2, 16-17 cm; slide 1; E.F. O 52/1.

Figure 2. Gen. and sp. indet. Sample 463-72-3, 14-15 cm; slide 3; E.F. U $32 / 3$.

Figure 3. ?Tripocyclia trigonum Rüst. Sample 463-87-1, 65-66 cm; slide 4; E.F. S 38/1.

Figure 4. Paronaella? diamphidia Foreman. Sample 463-84-1, 5-6 cm; slide 4; E.F. D 43/3.
Figure 5. Spongodiscus $\mathrm{sp} .(\times 100)$. Sample 466-34-2, 80-81 cm; slide 1 ; E.F. W $47 / 3$.

Figure 6. Gen. and sp. indet. Sample 466-34-2, 80-81 cm; slide 2; E.F. L $31 / 4$.

Figure 7. Gen. and sp. indet. Sample 465A-29-1, 43-44 cm; slide 14; E.F. N 27/3.

Figures 8a, b. Cromyodruppa concentrica Lipman. Sample 465A-291, 43-44 cm; slide 15; E.F. U 32/2.

Figure 9. Spongodiscus renillaeformis Campbell and Clark. Sample 466-34-2, 16-17 cm; slide 1; E.F. C 39/2. 


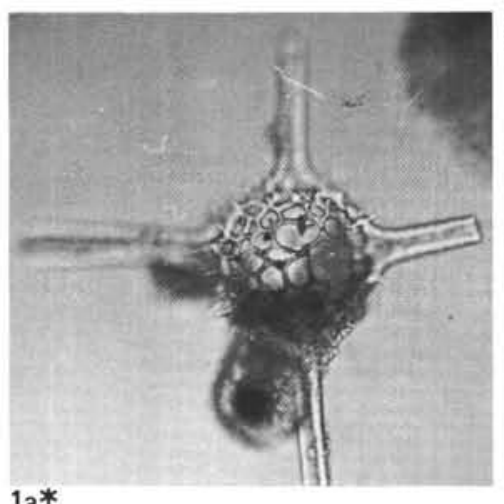

$1 a^{*}$
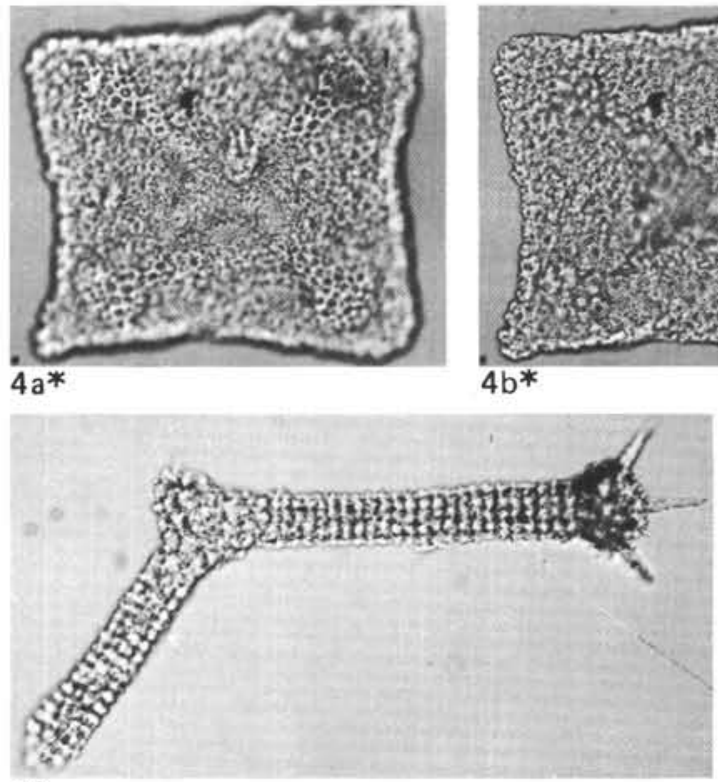

5*

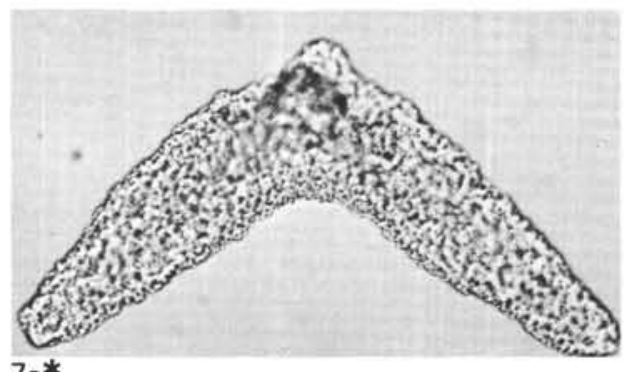

$7 a^{*}$

Plate 14. Photomicrographs.

Figures 1a, b. Hexastylurus magnificus (Squinabol) $(\times 100)$. Sample 463-89-1, 94-95 cm; slide 7; E.F. K 28/2.

Figure 2. Stylosphaera macrostyla Rüst $(\times 100)$. Sample 463-75-1, 21-22 cm; slide 26; E.F. O 29/1.

Figures 3a, b. Pseudoaulophacus? sulcatus (Rüst). Sample 463-75-1, 21-22 cm; slide 20; E.F. J 49/2.

Figures $4 a$, b. ?Stephanastrum inflexum Rüst $(\times 100)$. Sample 463-751, 21-22 cm; slide 15; E.F. V 34/1.
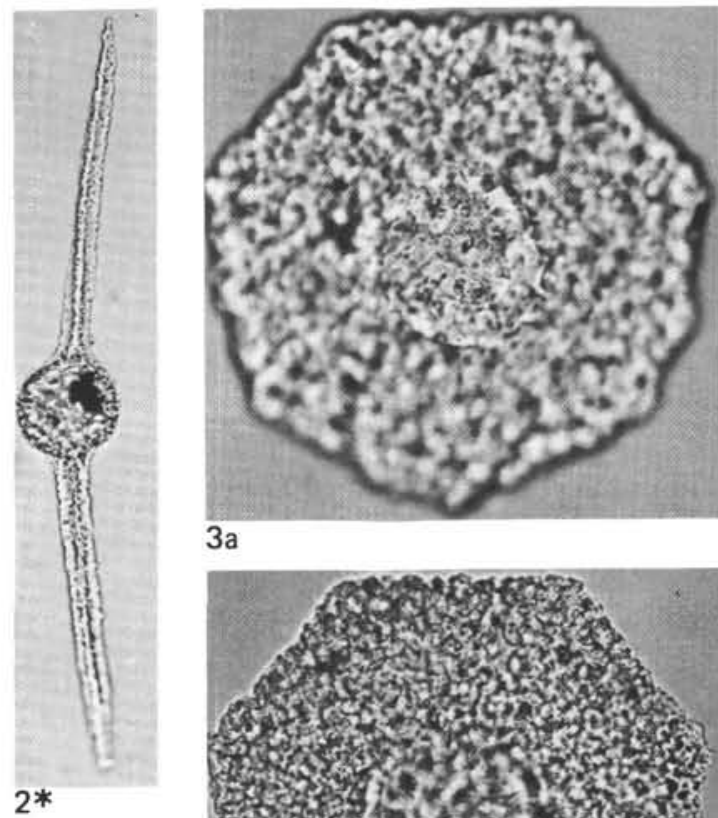

3a

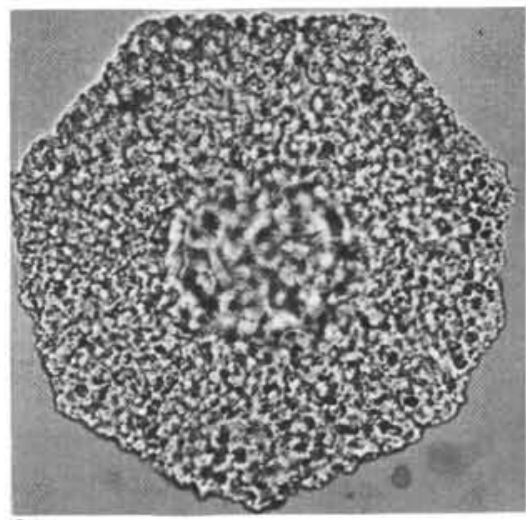

$3 b$

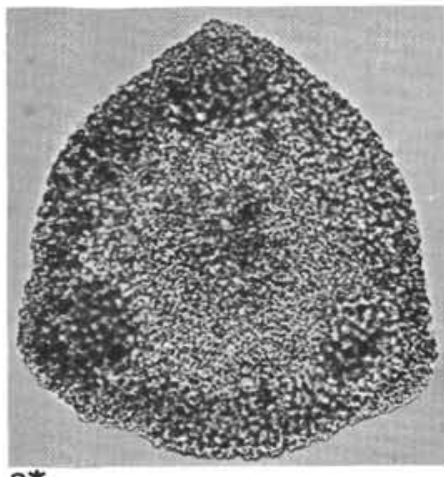

8*
Figure 5. Homoeoparonaella tricuspidata (Rüst) $(\times 100)$. Sample 46375-1, 21-22 cm; slide 26; E.F. S 29/1.

Figure 6. Hexinastrum cretaceum Lipman $(\times 100)$. Sample 463-75-1, 21-22 cm; slide 15; E.F. S 31/3.

Figures 7a, b. Gen. and sp. indet. $(\times 100)$. Sample 463-75-1, 21-22 cm; slide 19; E.F. F 29/1.

Figure 8. Cyclastrum infundibuliforme Rüst $(\times 100)$. Sample 46384-1, 5-6 cm; slide 6; E.F. J 38/4. 

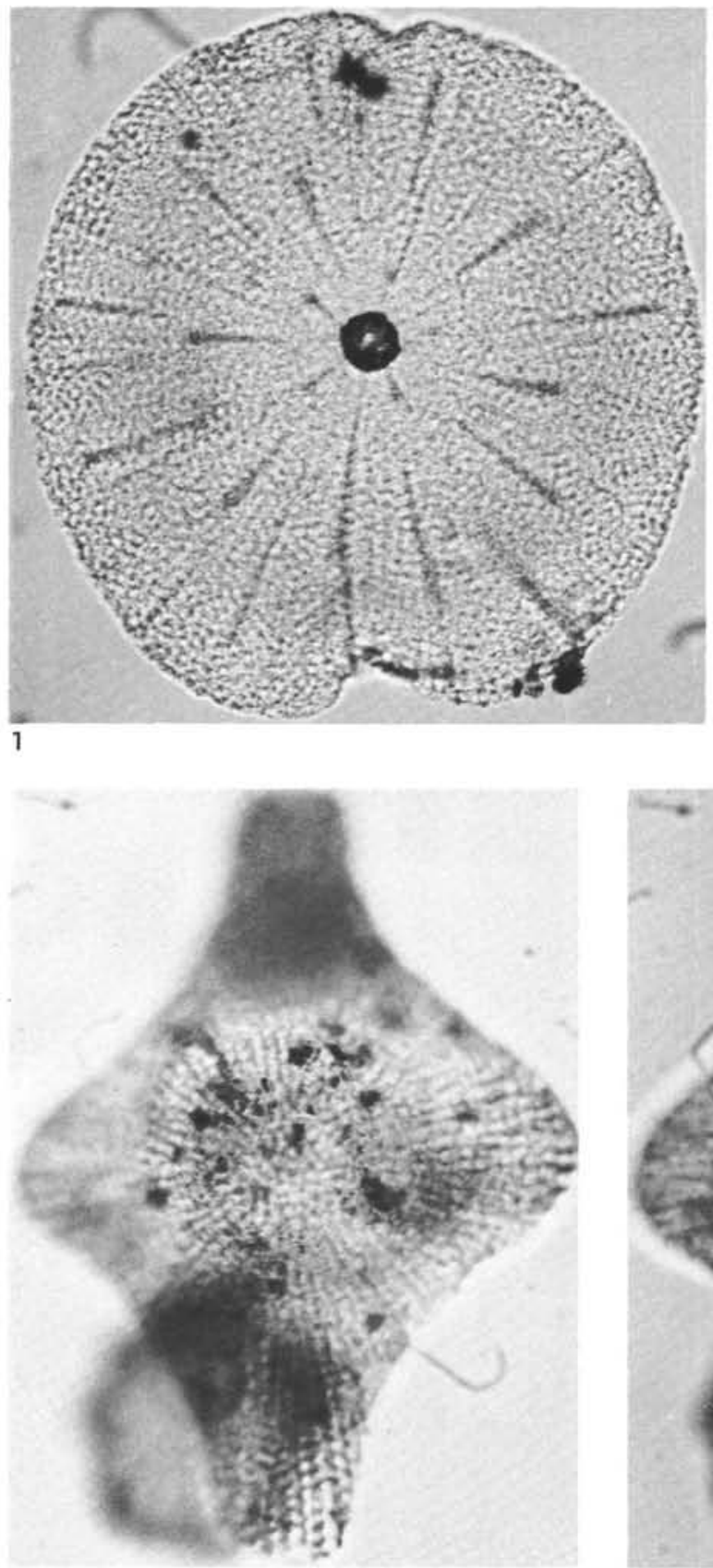

$4 a$

Plate 15. Photomicrographs.

Figure 1. Spongodiscus renillaeformis Campbell and Clark. Sample 466-34-2, 16-17 cm; slide 5; E.F. C 42/4.

Figure 2. Acaeniotyle diaphorogona Foreman. Sample 463-75-1, 21$22 \mathrm{~cm}$; slide 35; E.F. T $47 / 3$.

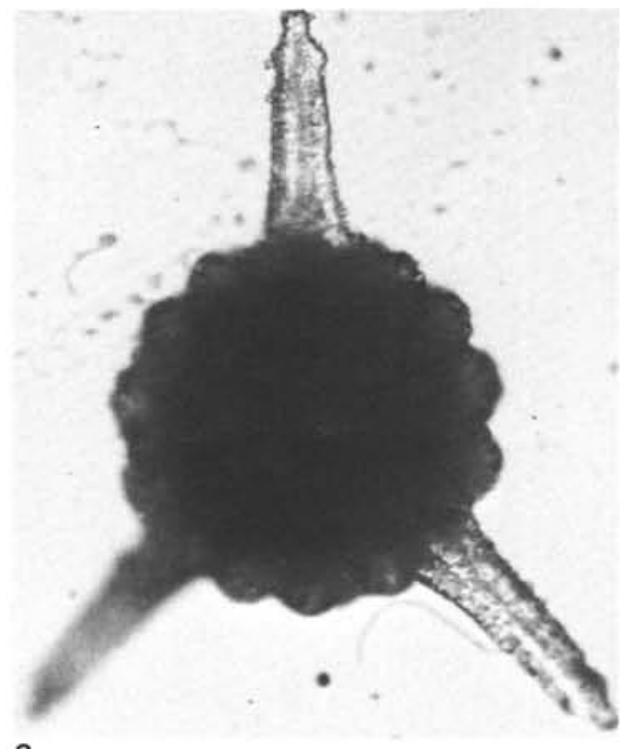

\section{2}
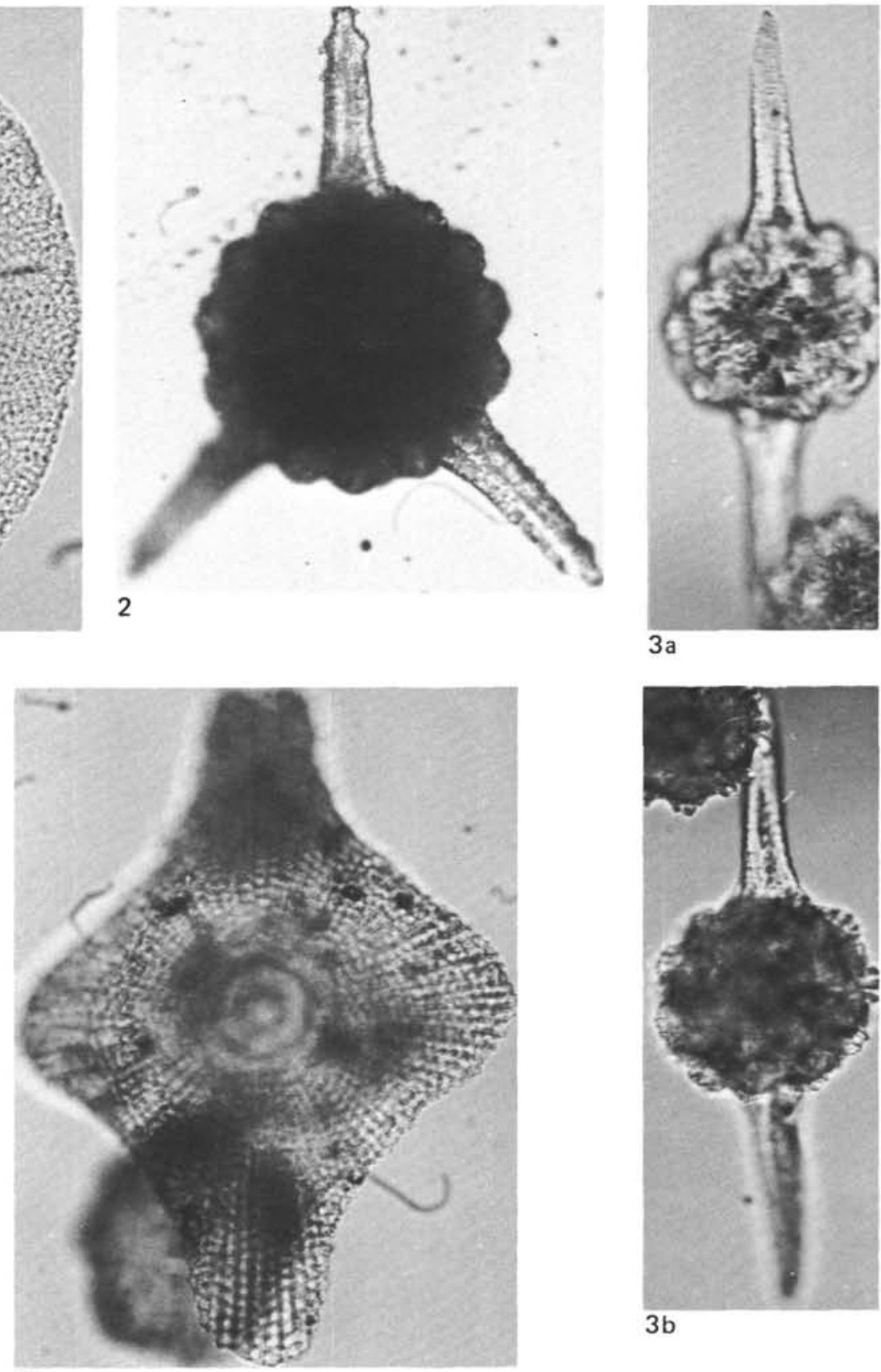

$3 a$

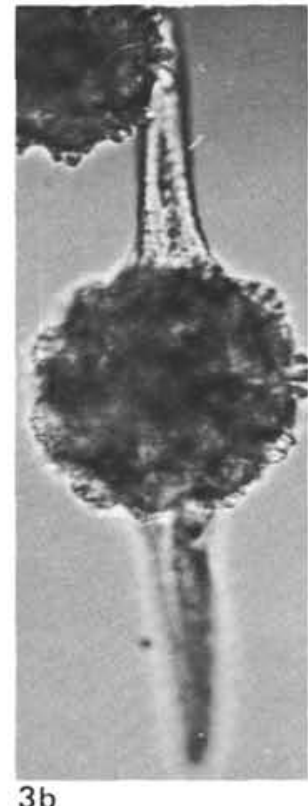

$3 b$

Figures 3a, b. Acaeniotyle umbilicata (Rüst). Sample 463-89-1, 23-24 cm; slide 4; E.F. J 34/3.

Figures 4a, b. Spongodruppa cocos Rüst. Sample 465A-29-1, 43-44 $\mathrm{cm}$; slide 13; E.F. T 27/1. 

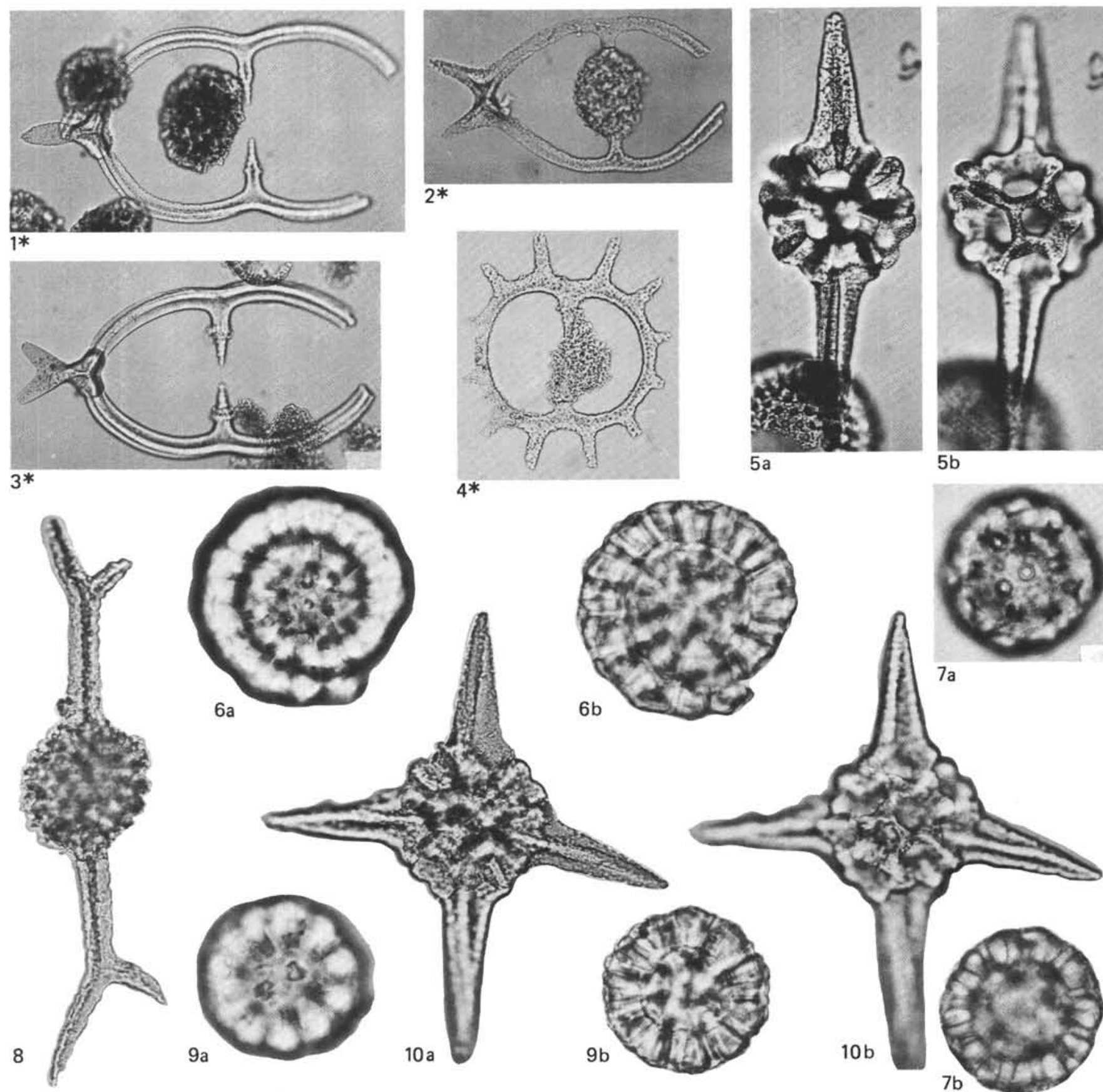

$5 a$

$5 b$

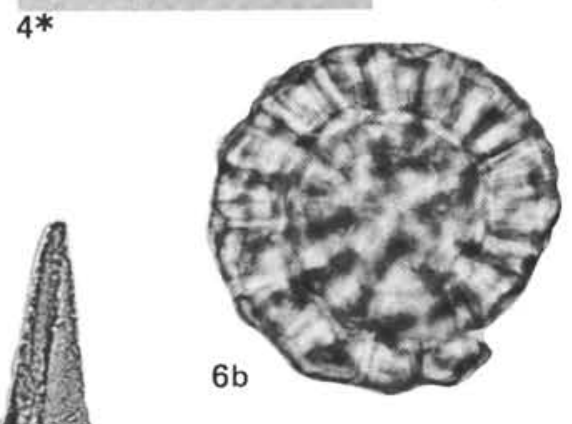

$6 b$

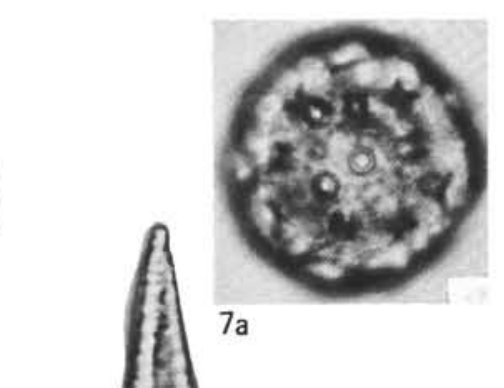

Plate 16. Photomicrographs.

Figure 1. Acanthocircus trizonalis (Rüst) $(\times 100)$. Sample 463-89-1, 23-24 cm; slide 5; E.F. Q 50/3.

Figure 2. Acanthocircus carinatus Foreman $(\times 100)$. Sample 463-751, 21-22 cm; slide 1; E.F. C 18/2.

Figure 3. Acanthocircus dicranacanthos (Squinabol) $(\times 100)$. Sample 463-89-1, 105-106 cm; slide 5; E.F. Q 37/2.

Figure 4. Spongosaturnalis horridus (Squinabol) group $(\times 100)$. Sample 463-75-1, 21-22 cm; slide 2; E.F. O 2/1

Figures 5a, b. Sphaerostylus lanceola (Parona). Sample 463-90,CC; slide 9; E.F. K 35/4.

Figures 6a, b. Stylochlamyum? sp. group. Sample 463-83-1, 38-39 $\mathrm{cm}$; slide 2; E.F. M 43/4.

Figure 7a, b. Gen. and sp. indet. Sample 463-74-1, 110-111 cm; slide 4; E.F. K 50/4.

Figure 8. Dicroa sp. A Foreman. Sample 463-75-1, 21-22 cm; slide 20; E.F. F 34/4.

Figures 9a, b. Gen. and sp. indet. Sample 463-77-1, 60-61 cm; slide 4; E.F. H $31 / 1$.

Figures $10 \mathrm{a}$, b. Staurosphaera septemporata Parona. Sample 46389-1, 23-24 cm; slide 2; E.F. D 36/3. 


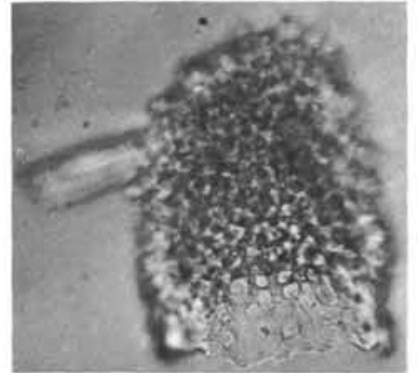

1a

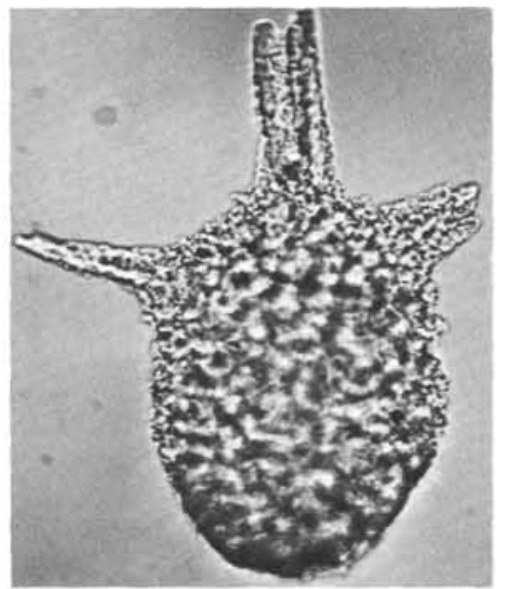

4

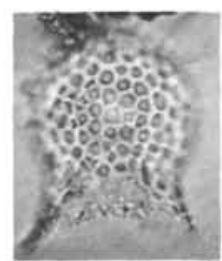

$6 a$
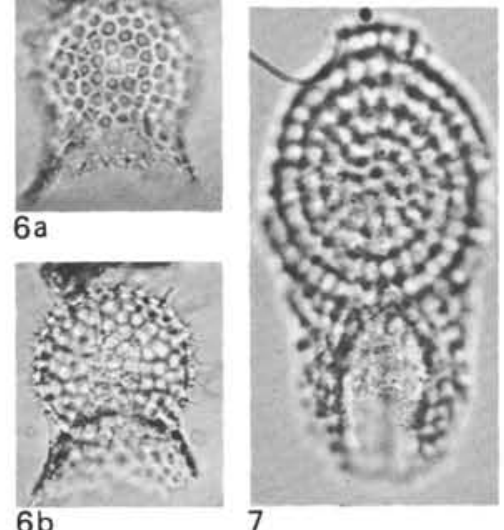

9

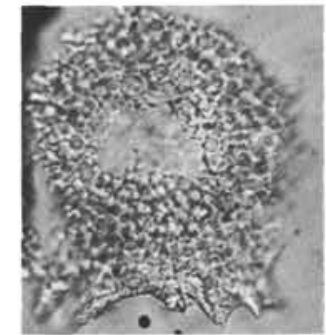

$5 a$

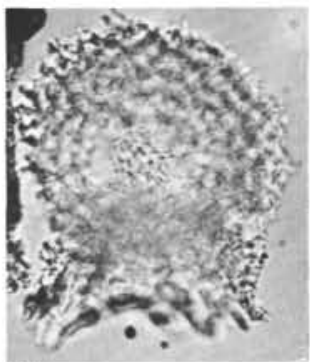

$5 b$

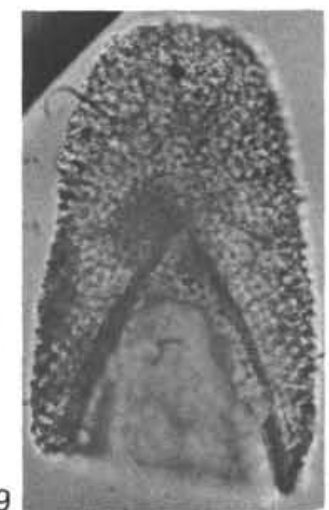

Plate 17. Photomicrographs.

Figures 1a, b. Spongopyle sp. Sample 463-89-1, 94-95 cm; slide 10; E.F. B $34 / 4$.

Figures 2a, b. Spongopyle ecleptos Renz. Sample 463-91,CC; slide 1; E.F. Z $42 / 3$

Figures 3a, b. Spongodiscus misele, n. sp. Holotype. Sample 46373-1, 18-19 cm; slide 4; E.F. U 34/1.

Figure 4. Gen. and sp. indet. Sample 463-75-1, 21-22 cm; slide 30; E.F. $\times 26 / 3$.
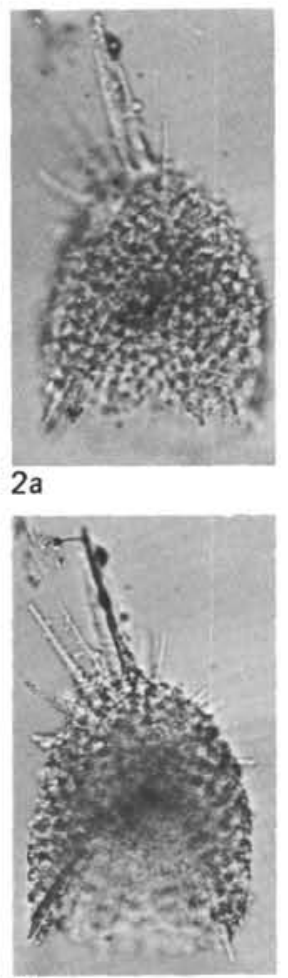

$2 \mathrm{~b}$

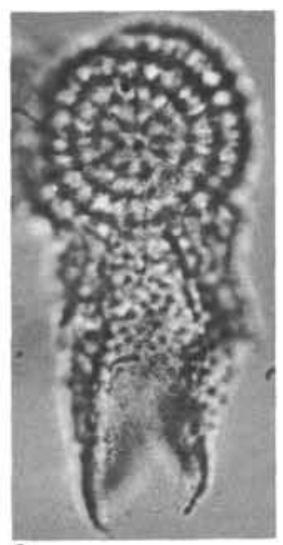

8

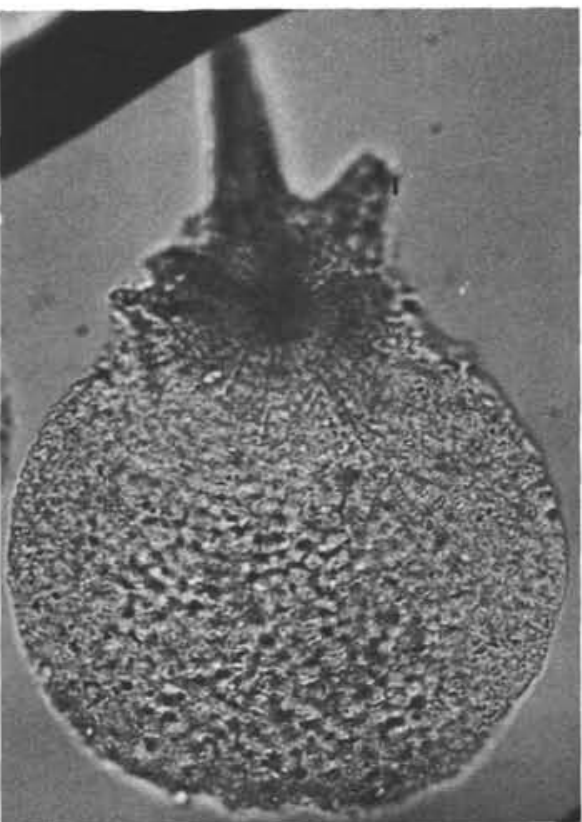

$3 a$

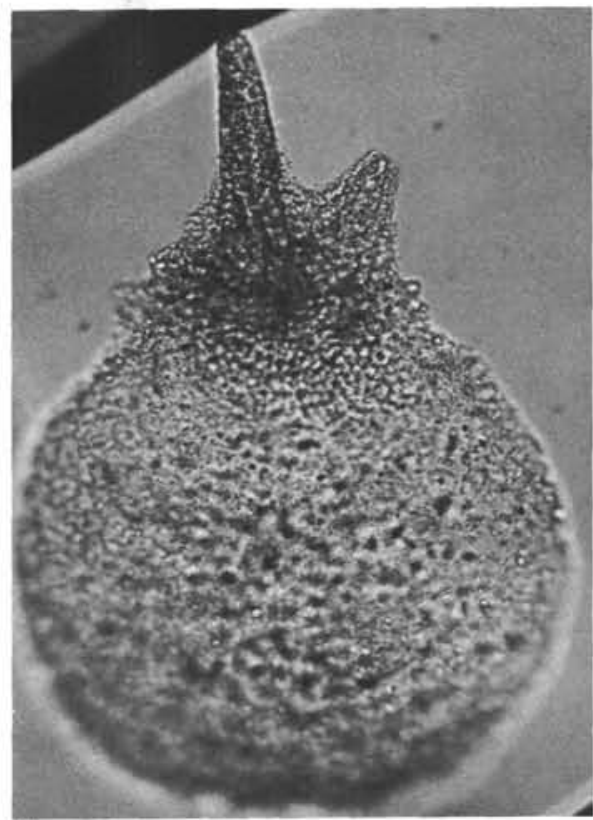

$3 b$

Figures $5 \mathrm{a}$, b. Sphaeropyle sp. aff. S. thirencis, n. sp. Sample 46390, CC; slide 10; E.F. V 37/2.

Figures 6a, b. Sphaeropyle thirencis, n. sp. Holotype. Sample 46389-1, 15-16 cm; slide 7; E.F. C 45/1.

Figure 7. Spongopyle insolita Kozlova. Spiral morphotype. Sample 466-34-2, 16-17 cm; slide 9; E.F. F 43/4.

Figure 8. Spongopyle insolita Kozlova. Concentric morphotype. Sample 466-34-2, 16-17 cm; slide 2; E.F. F 47/1.

Figure 9. Spongopyle ecleptos Renz. Sample 465A-29-1, 43-44 cm; slide $13 ;$ E.F. E $31 / 4$. 


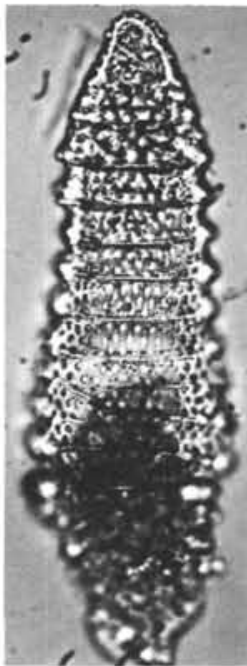

1a

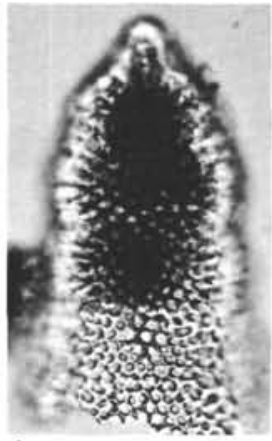

$4 a$

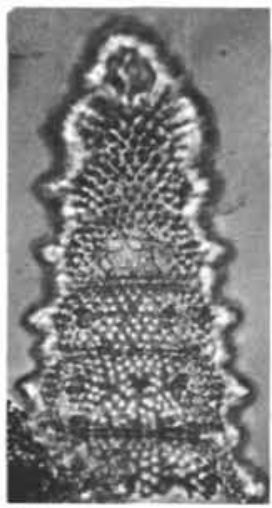

7a

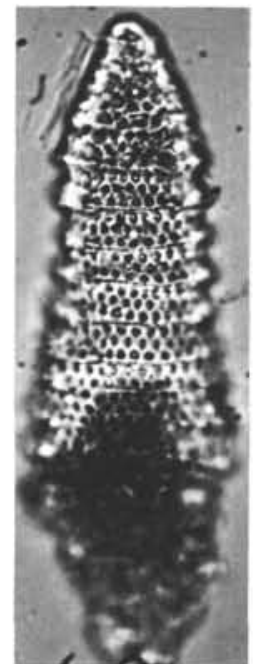

$1 \mathrm{~b}$

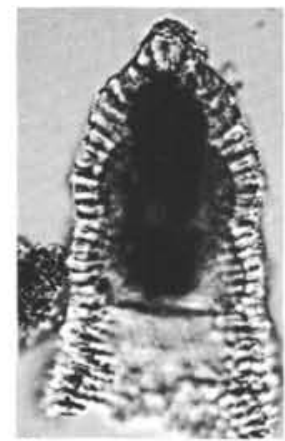

$4 b$

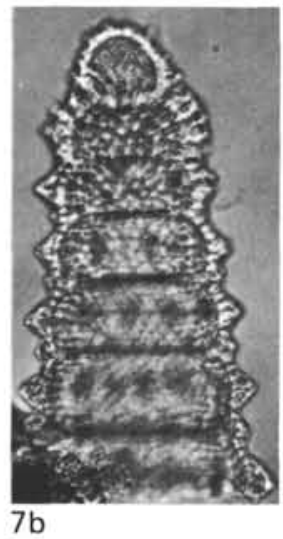

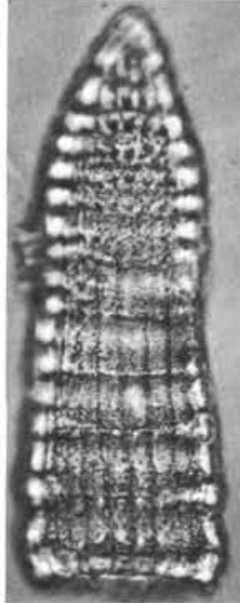

2a

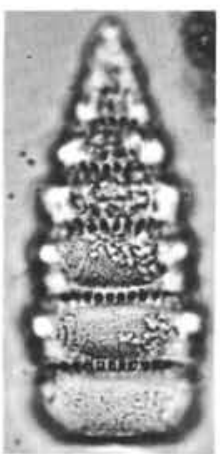

$5 a$

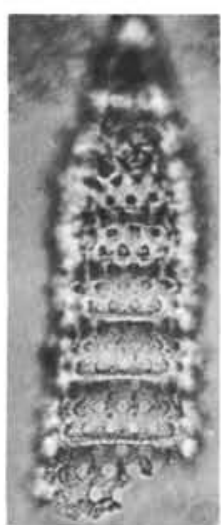

$8 a$

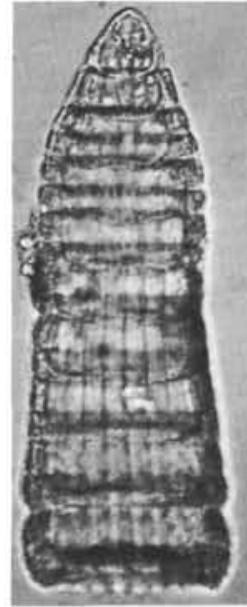

$2 b$

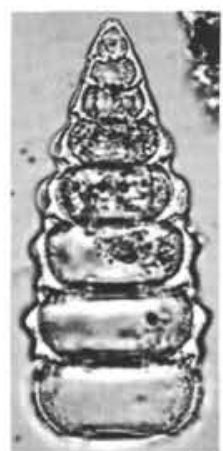

$5 b$

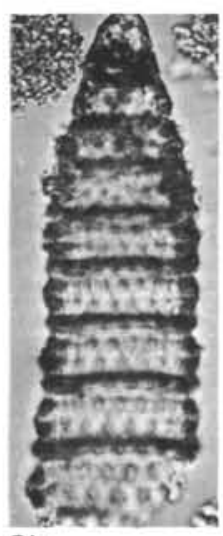

$8 b$

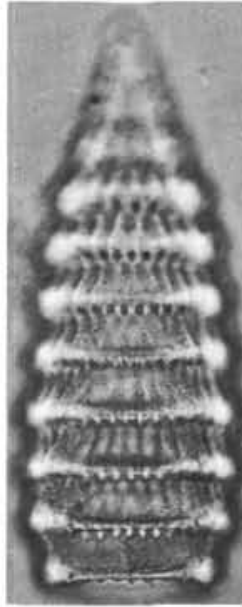

$3 a$

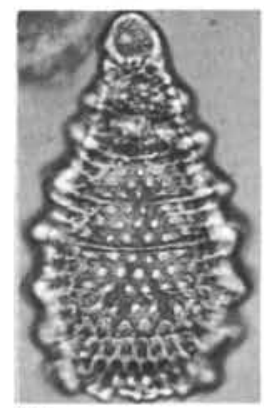

$6 a$

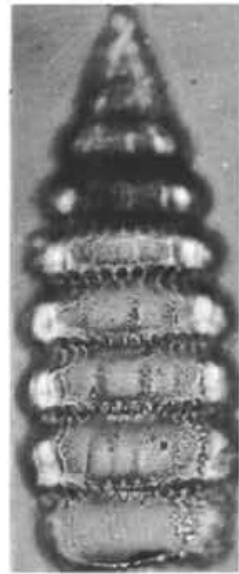

9 a

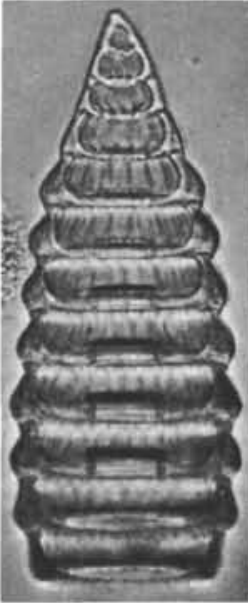

3b

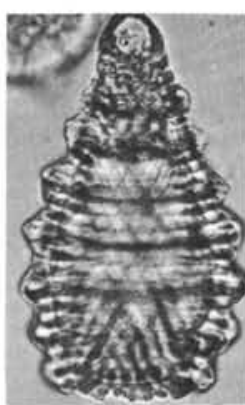

$6 b$

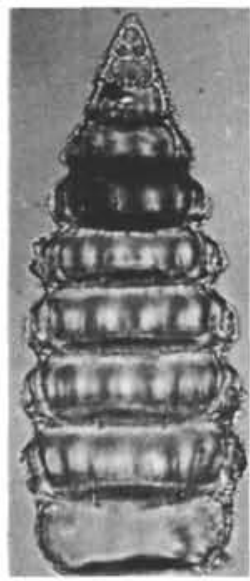

$9 b$

Plate 18. Photomicrographs.

Figures 5a, b. Pseudodictyomitra lilyae (Tan Sin Hok). Sample 46390,CC; slide 8; E.F. P 38/4.

Figure 6a, b. Parvicingula boesii (Parona). Sample 463-89-1, 105-106 $\mathrm{cm}$; slide 3; E.F. G 41/2.

Figures 7a, b. Xitus sp. A. Sample 463-90,CC; slide 20; E.F. T 47/3.

Figures $8 \mathrm{a}$, b. Gen. and sp. indet. Sample 463-89-1, 94-95 cm; slide 8; E.F. U 44/2

Figures 9a, b. Pseudodictyomitra lanceloti, n. sp. Holotype. Sample 463-89-1, 94-95 cm; slide 10; E.F. X 43/2. 


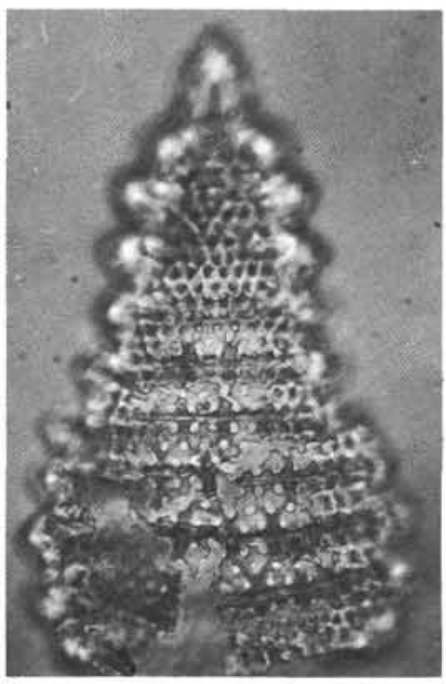

1a

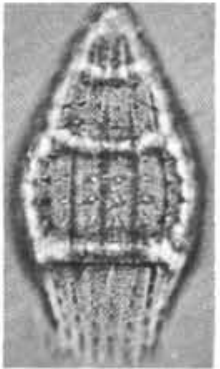

$3 a$

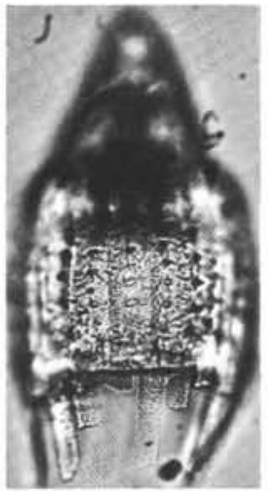

$7 a$

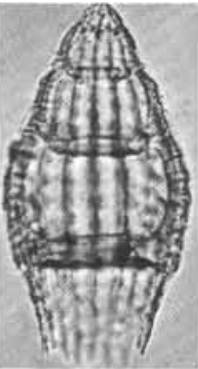

$3 \mathrm{~b}$

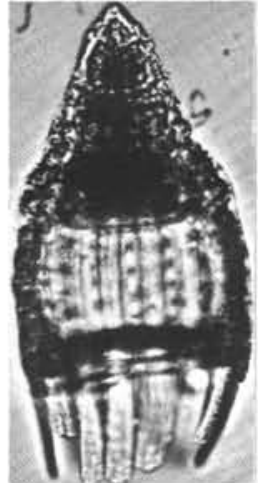

$7 b$

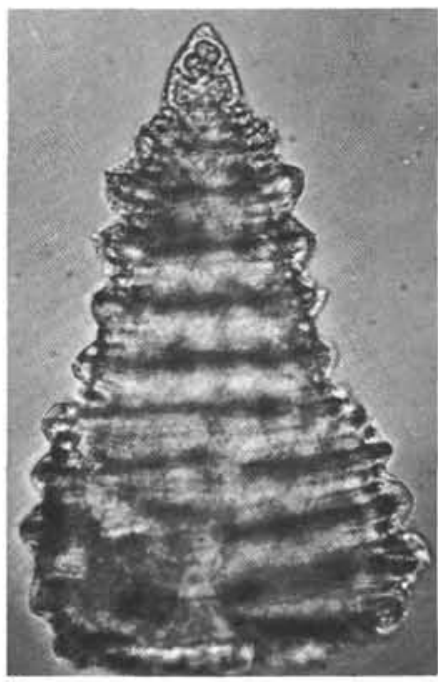

$1 b$

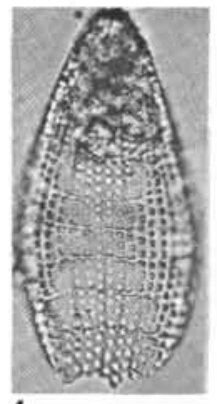

4

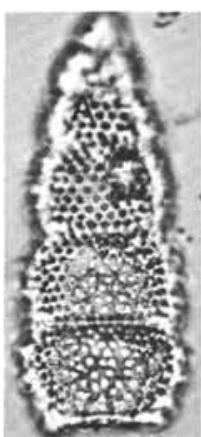

$5 a$

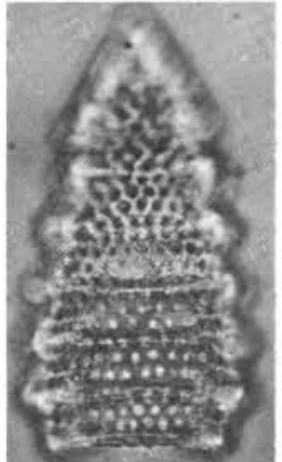

$8 a$

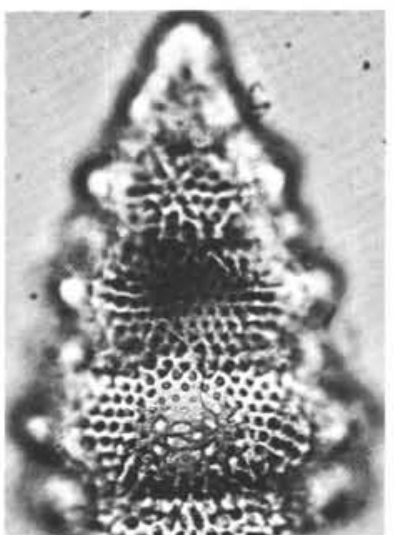

$2 a$

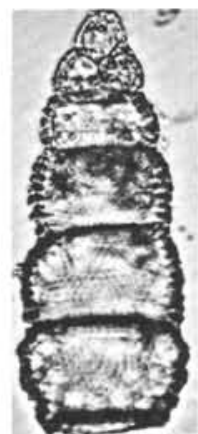

$5 b$

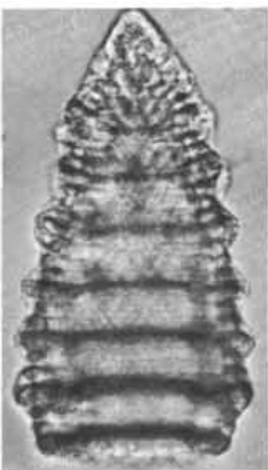

$8 b$

$6 a$

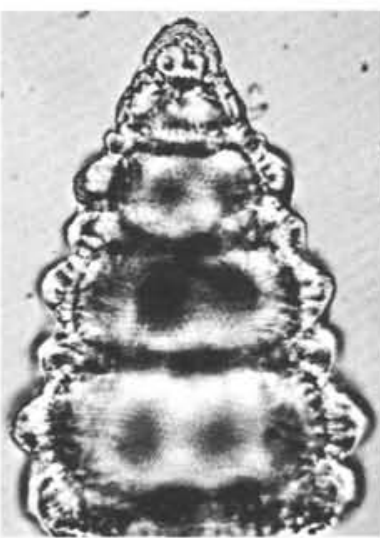

$2 b$
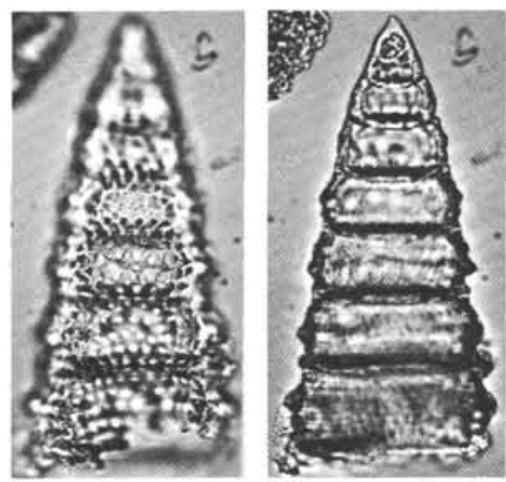

$6 b$

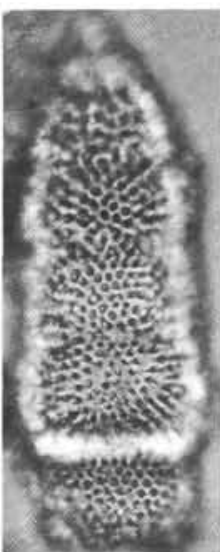

$9 \mathrm{a}$

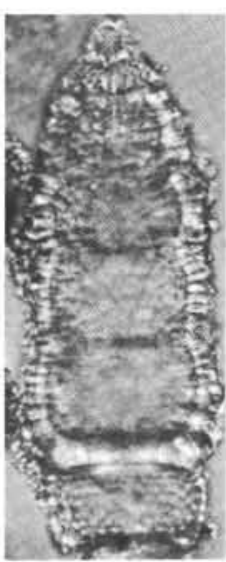

$9 b$
Plate 19. Photomicrographs.

Figures 1a, b. Xitus alievi (Foreman). Sample 463-90,CC; slide 20; E.F. $44 / 4$.

Figures 2a, b. Xitus spicularius (Aliev). Sample 463-90,CC; slide 7; E.F. C $42 / 1$.

Figures 3a, b. Archaeodictyomitra brouweri var. $\alpha$ (Tan Sin Hok). Sample 463-92-1, 10-11 cm; slide 3; E.F. M 27/2.

Figure 4. Hsuum sp. cf. H. obispoensis Pessagno. Sample 463-90,CC; slide 17 ; E.F. H $38 / 1$.

Figures 5a, b. Lithomitra? pseudopinguis Tan Sin Hok. Sample 46390,CC; slide 6; E.F. L 50/4.
Figures 6a, b. Xitus vermiculatus (Renz). Sample 463-90,CC; slide 5; E.F. E $46 / 4$.

Figures 7a, b. Thanarla pulchra (Squinabol). Sample 463-90,CC; slide 4; E.F. C $48 / 4$.

Figures $8 \mathrm{a}$, b. Xitus alievi (Foreman). Some specimens like this have a thick wall on the three first segments. Sample 463-891, 23-24 cm; slide 5 ; E.F. F $35 / 4$.

Figures 9a, b. Lithomitra? pseudopinguis Tan Sin Hok. Sample 46389-1, 94-95 cm; slide 7; E.F. G 28/1. 


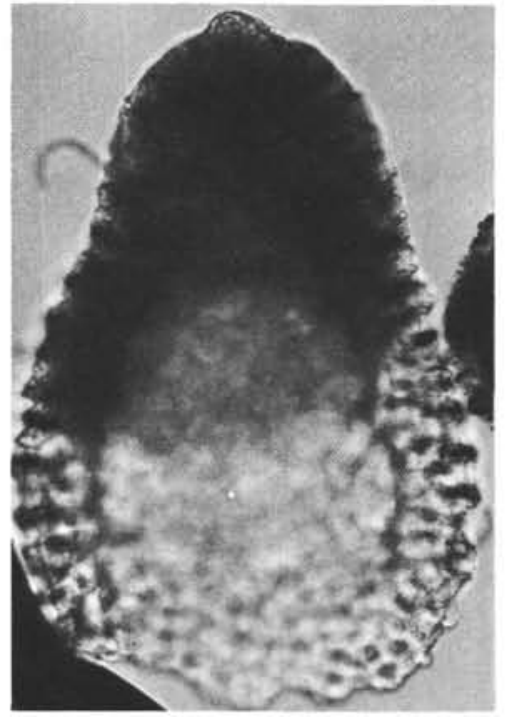

1a

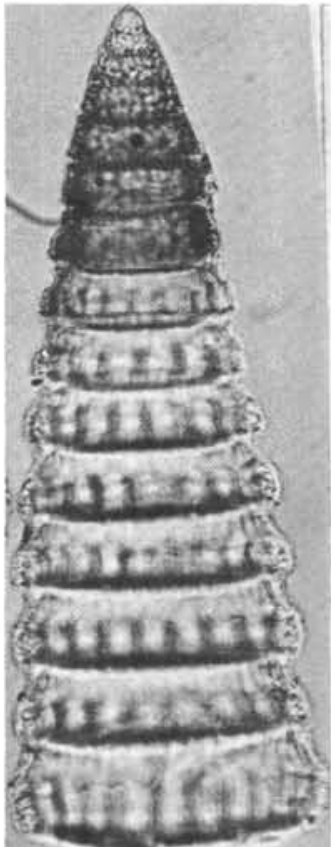

$4 a$

$4 b$

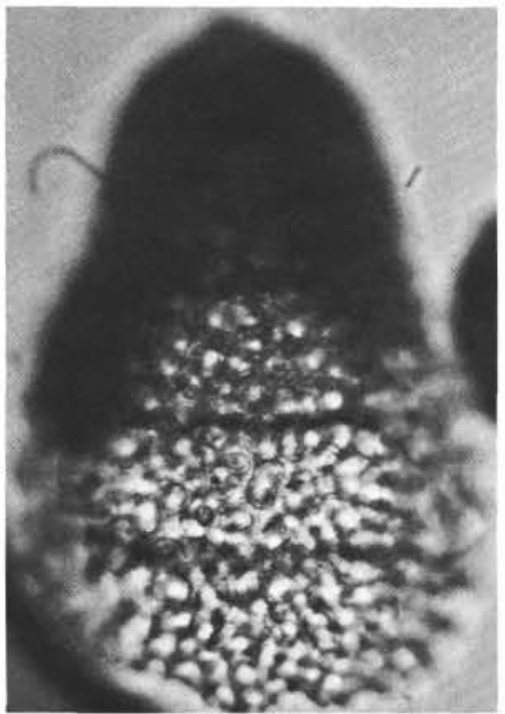

$1 b$

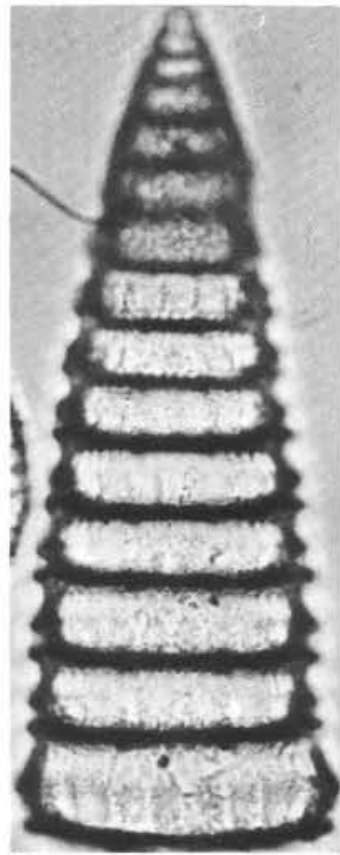

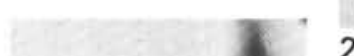

2
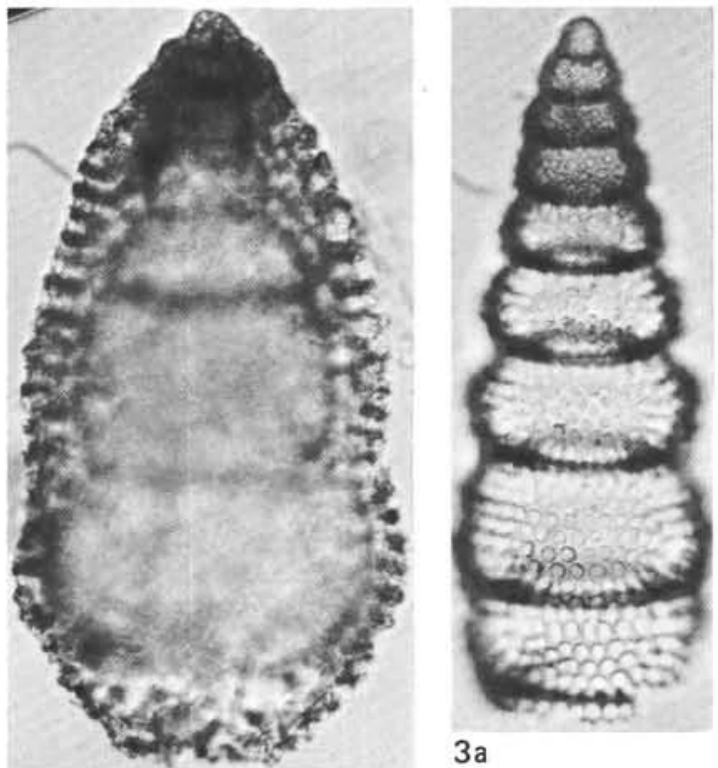

$3 a$

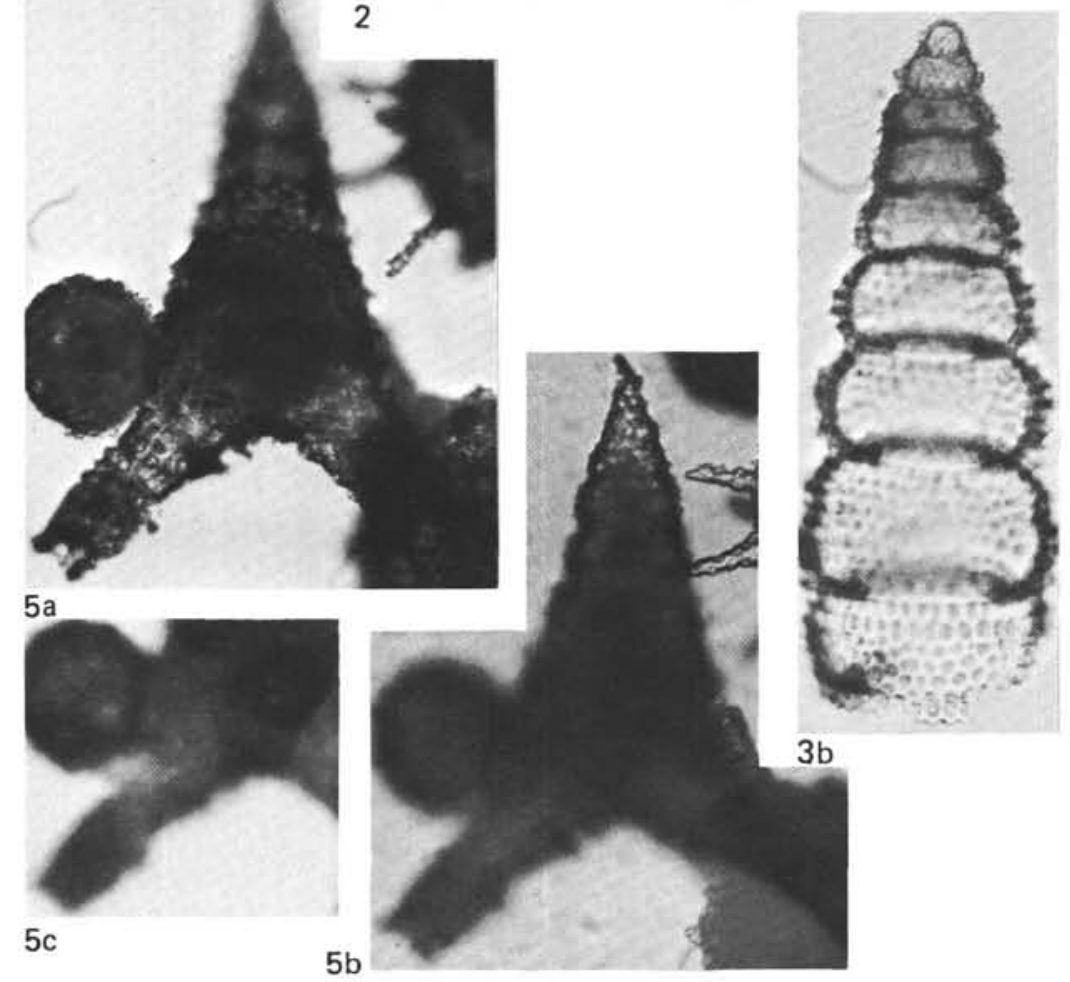

Figures 3a, b. Parvicingula tekschaensis (Aliev). Sample 465A-29-1, 43-44 cm; slide 15; E.F. W 42/1.

Figures 4a, b. Pseudodictyomitra carpatica (Lozyniak). Sample 46634-2, 16-17 cm; slide 10; E.F. D 47/2.

Figures 5a-c. Crolanium pythiae, n. sp. Sample 463-75-1, 21-22 cm; slide 35 ; E.F. D 36/1.
Plate 20. Photomicrographs.

Figures 1a, b. Obesacapsula somphedia (Foreman). Sample 465A29-1, 43-44 cm; slide 1; E.F. T 27/3.

Figure 2. Obesacapsula somphedia (Foreman). Sample 465A-29-1; 43-44 cm; slide 1; E.F. T 34/2. 


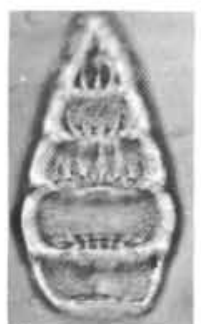

1a

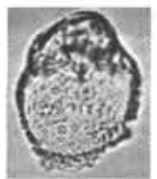

$6 a$

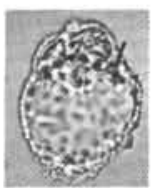

$6 b$

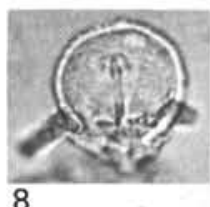

8

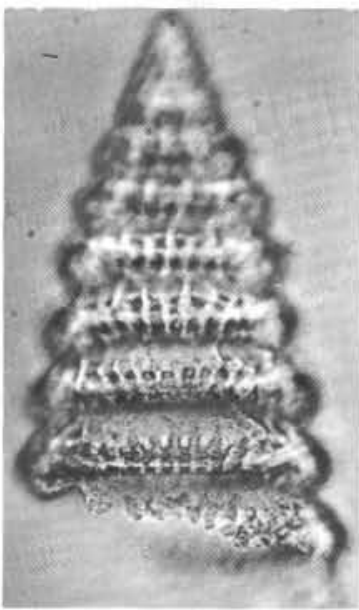

$11 \mathrm{a}$

$1 \mathrm{~b}$

$7 b$
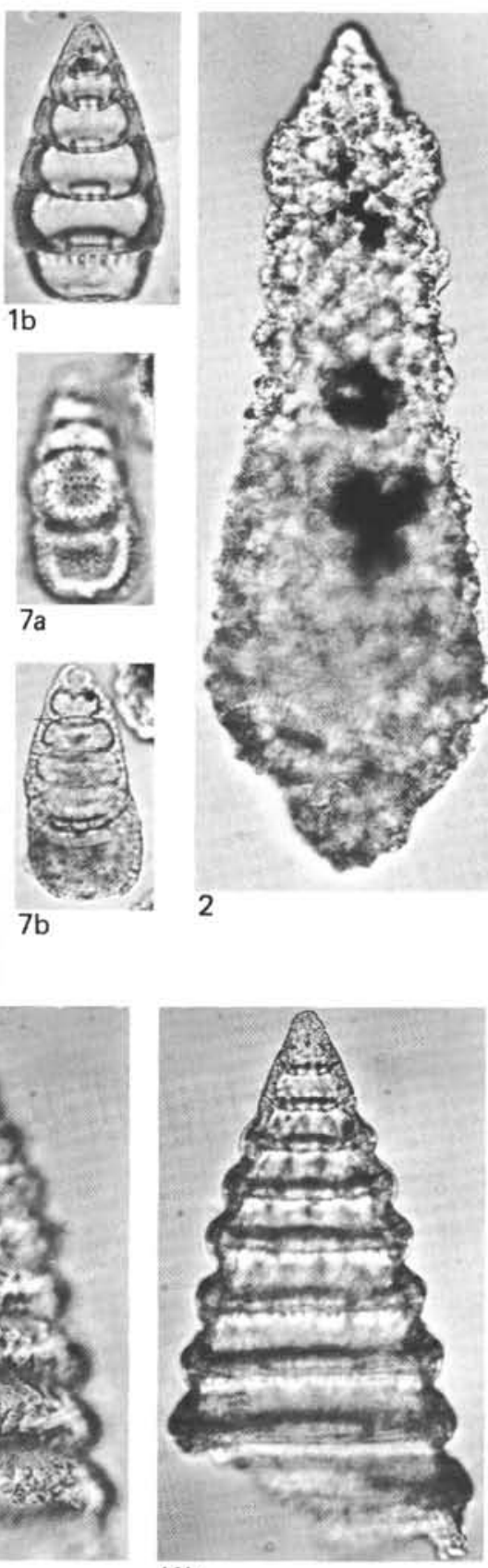

$11 \mathrm{~b}$

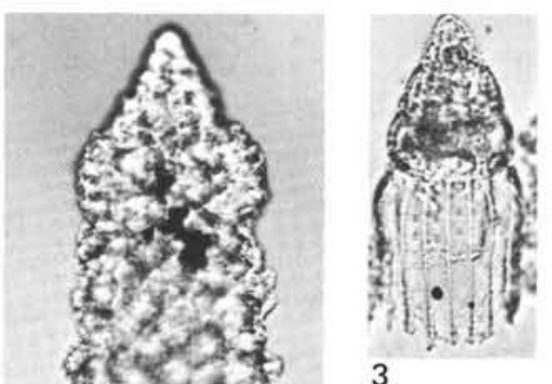

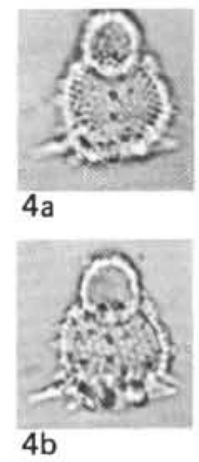

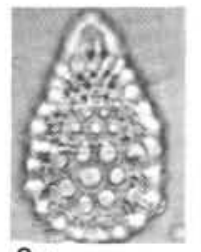

9a

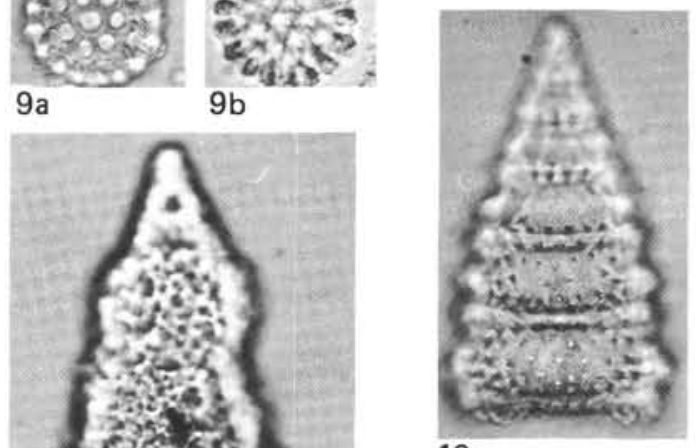

$10 a$

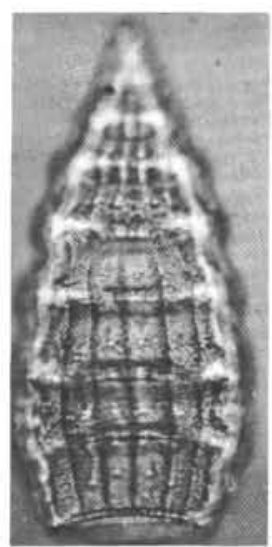

$13 a$

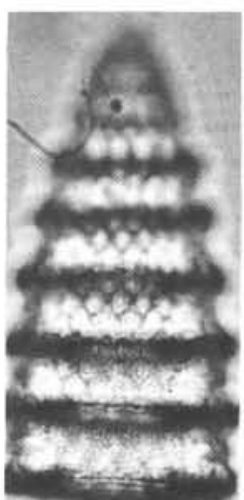

$5 a$

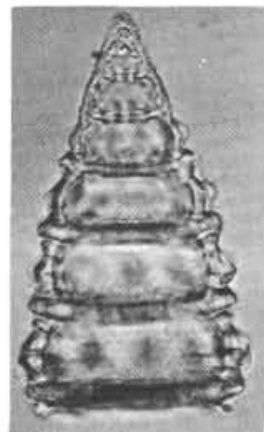

$10 \mathrm{~b}$

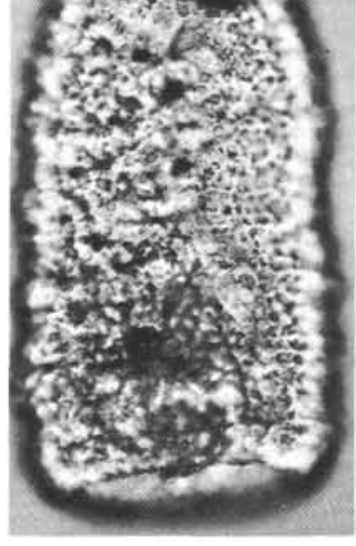

12

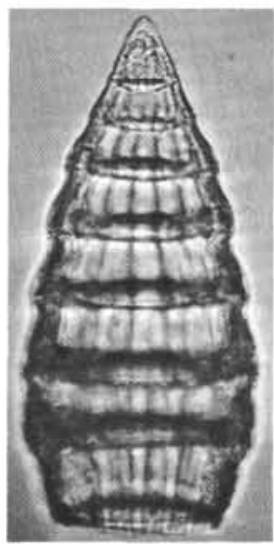

$13 b$

Plate 21. Photomicrographs.

Figures 1a, b. Pseudodictyomitra blabla, n. sp. Holotype. Sample 463-86, CC; slide 4; E.F. Y 31/4.

Figure 2. ?Parvicingula malleola (Aliev). Sample 463-75-1, 21-22 cm; slide 19; E.F. K 39/4.

Figure 3. Thanarla karpoffae, n. sp. Holotype. Sample 463-90,CC; slide 10; E.F. U 26/0.

Figures 4a, b. Gen. and sp. indet. Sample 463-87-1, 65-66 cm; slide 2; E.F. $40 / 1$.

Figures 5a, b. Lithostsrobus punctulatus Pessagno. Sample 466-34-2, 80-81 cm; slide 3; E.F. U 39/0.

Figures 6a, b. Lophophaena sp. Sample 463-85-1, 142-143 cm; slide 1; E.F. G 27/2.

Figures 7a, b. Solenotryma sp. Sample 463-82,CC; slide 2; E.F. W $39 / 3$.

Figure 8. Saitoum cepeki, n. sp. Holotype. Sample 463-86,CC; slide 4 ; E.F. K 43/3.

Figures 9a, b. Gen. and sp. indet. Sample 463-88-1, 52-53 cm; slide 5; E.F. M 45/2.

Figures 10a, b. Xitus sp. indet. Sample 463-86,CC; slide 5; E.F. O $35 / 1$.

Figures 11a, b. Archaeodictyomitra puga, n. sp. Holotype. Sample 463-89-1, 15-16 cm; slide 8; E.F. D 40/1.

Figure 12. Parvicingula malleola (Aliev). Sample 463-75-1, 21-22 cm; slide 23; E.F. F 34/2.

Figures 13a, b. Archaeodictyomitra pseudoscalaris (Tan Sin Hok). Sample 463-87-1, 65-66 cm; slide 5; E.F. L 37/3. 


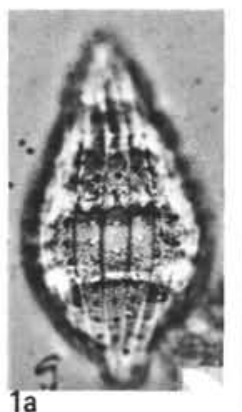

4
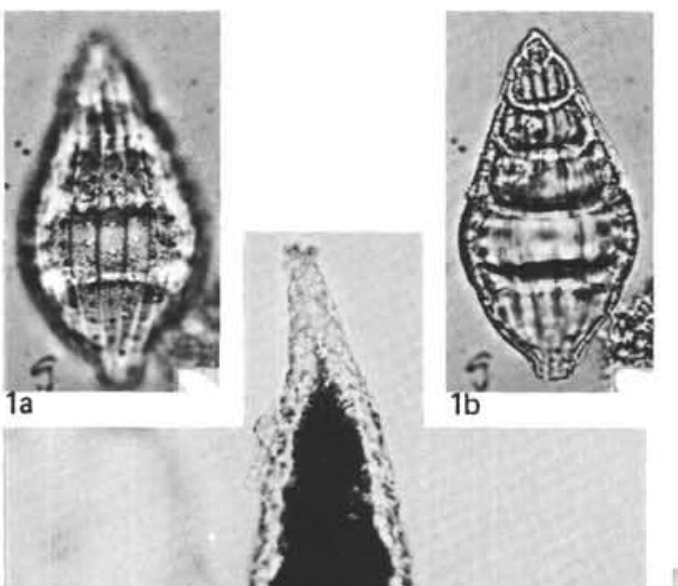$$
\text { s. }
$$

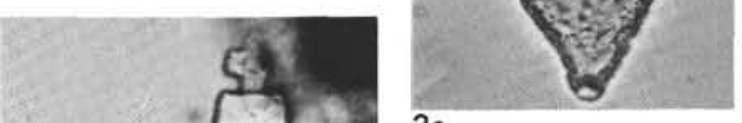

3a
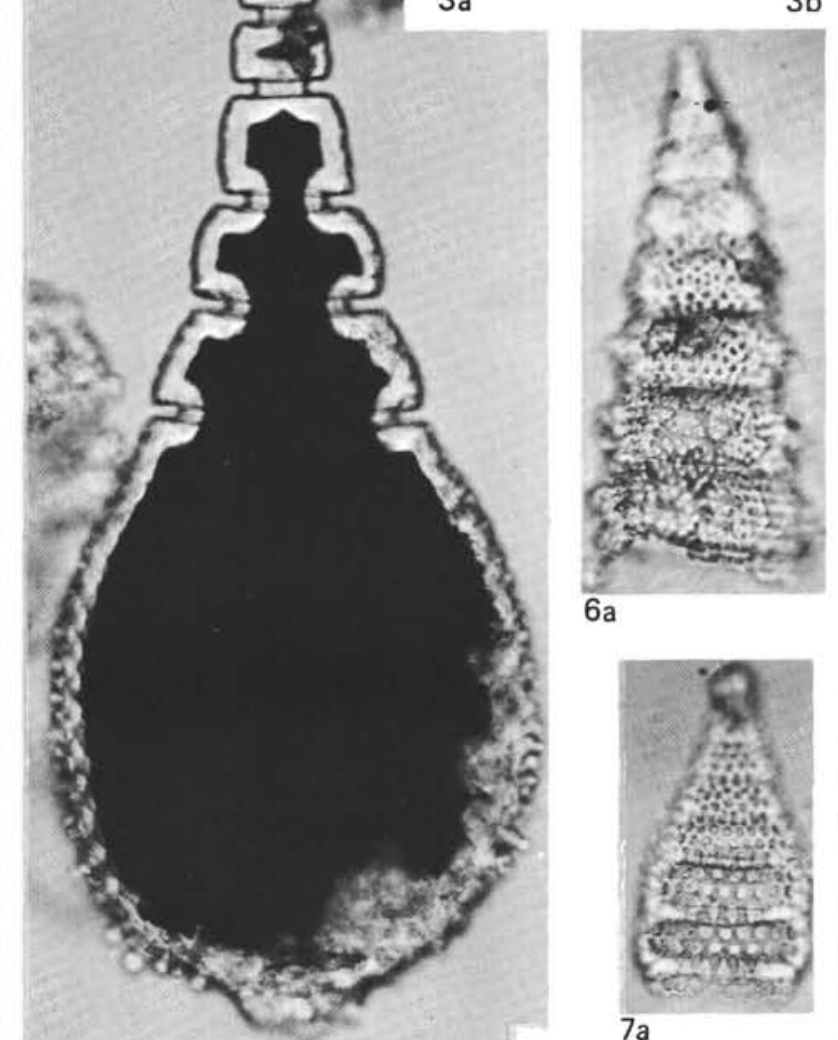

$7 a$

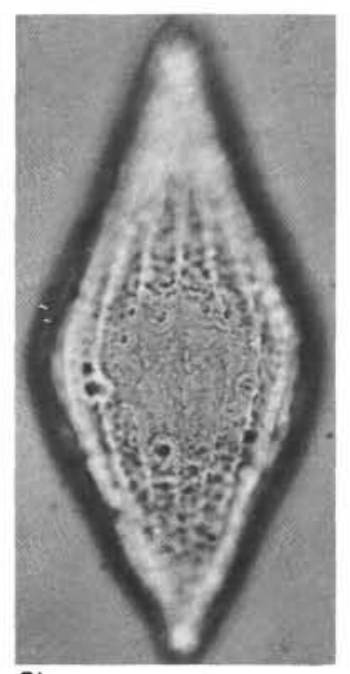

$3 b$

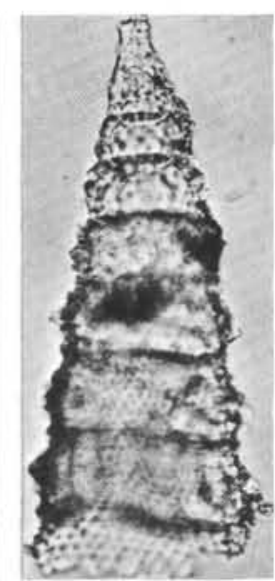

$6 \mathrm{~b}$

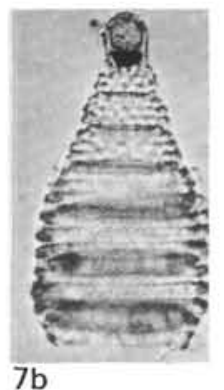

Figure 4. Archicapsa similis Parona. Sample 463-75-1, 21-22 cm; slide 13; E.F. E $8 / 3$.

Figure 5. Archicapsa similis Parona. Sample 463-75-1, 21-22 cm; slide 9; E.F. D $24 / 3$.

Figures 6a, b. Stichomitra asymbatos Foreman. Sample 463-89-1, 105-106 cm; slide 11; E.F. C 56/4.

Figures 7a, b. Amphipyndax mediocris (Tan Sin Hok). Sample 46389-1, 94-95 cm; slide 6; E.F. C 26/3. 


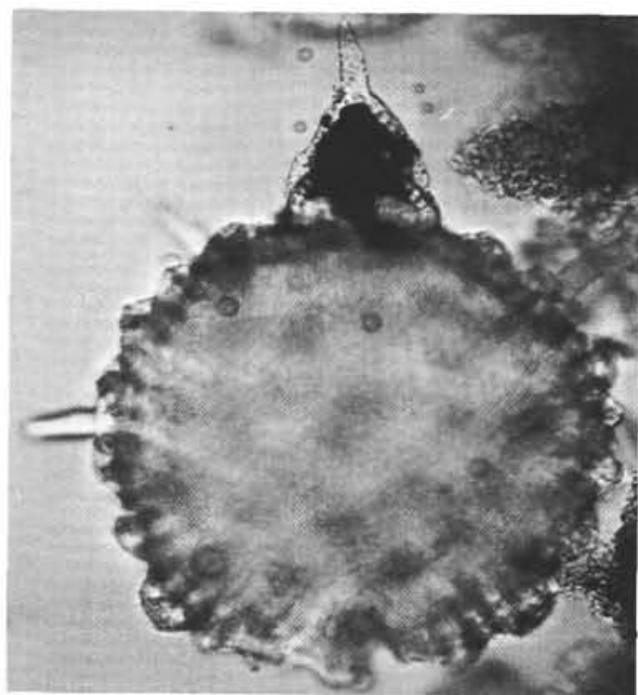

$1 a$

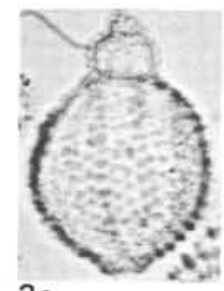

$3 a$

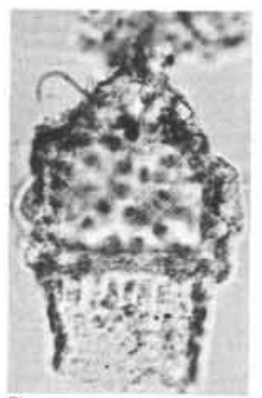

5

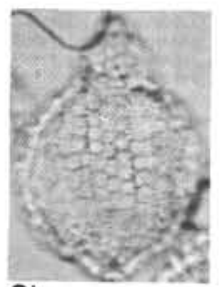

$3 b$

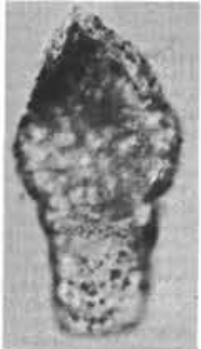

2a

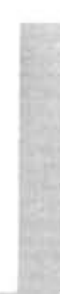

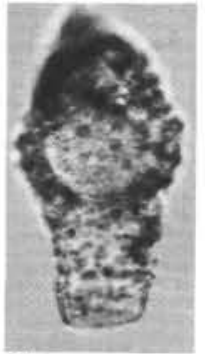

$2 b$

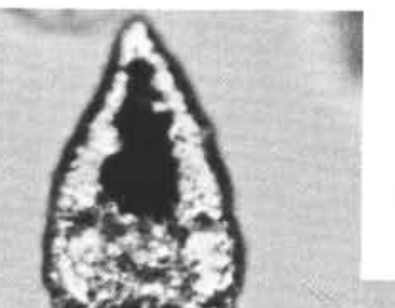

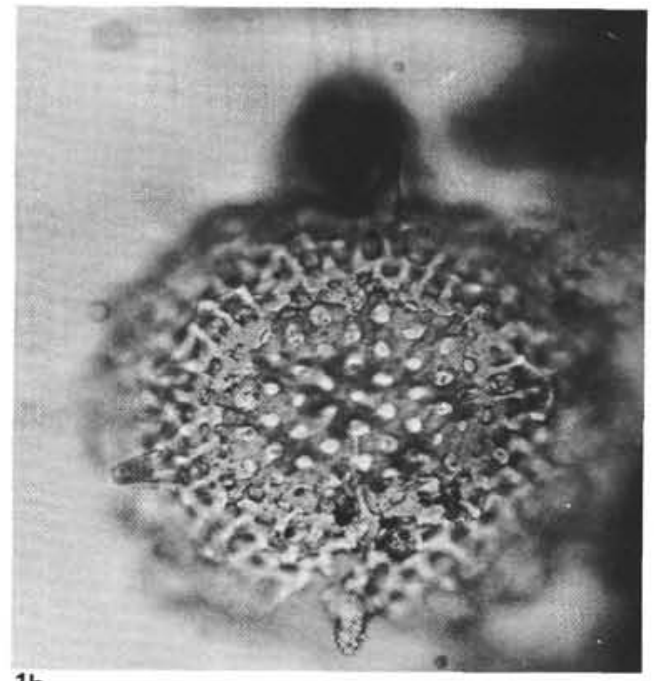

1b

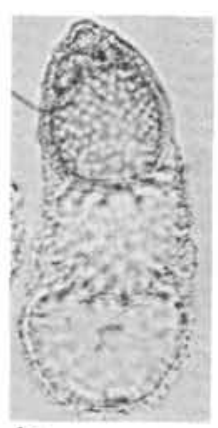

$4 a$

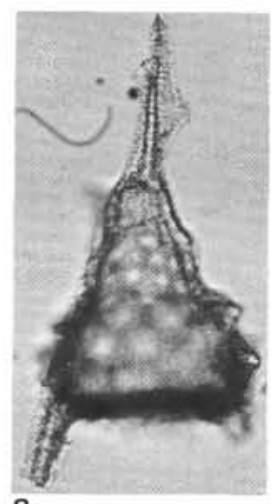

$8 a$

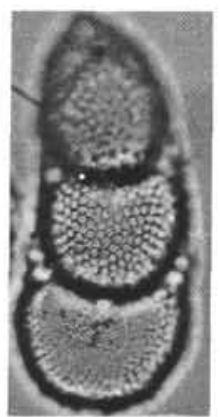

$4 b$

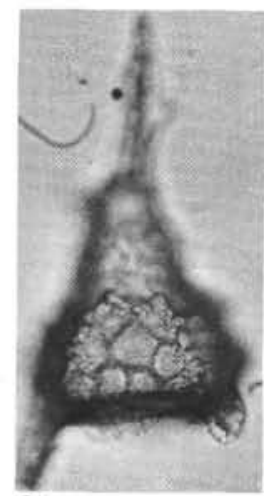

$8 b$
Plate 23. Photomicrographs.

Figures 1a, b. Sethocapsa trachyostraca Foreman. Sample 463-89-1, 94-95 cm; slide 5; E.F. K $31 / 1$.

Figures 2a, b. Rhopalosyringium sp. indet. Sample 466-29-1, 43-44 cm; slide 3; E.F. G 47/4.

Figures 3a, b. Gen. and sp. indet. Sample 466-34-2, 16-17 cm; slide 3; E.F. H $49 / 3$.

Figures $4 \mathrm{a}$, b. Solenotryma sp. indet. Sample 466-34-2, 16-17 cm; slide 3; E.F. Y $46 / 2$.
Figure 5. Rhopalosyringium majuroensis, n. sp. Sample 465A-29-1, 43-44 cm; slide 11; E.F. H 38/3.

Figure 6a, b. Tripocalpis ellyae Tan Sin Hok. Sample 463-89-1, 23-24 cm; slide 8; E.F. E 24/2.

Figure 7. Archicapsa similis Parona. Sample 463-62-1, 148-149 cm; slide 4; E.F. J 44/3.

Figures 8a, b. Ultranapora durhami Pessagno. Sample 466-29-1, 43$44 \mathrm{~cm}$; slide 11; E.F. M 45/2. 


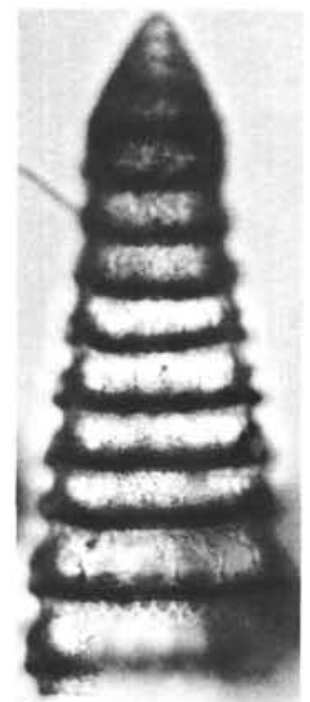

1a

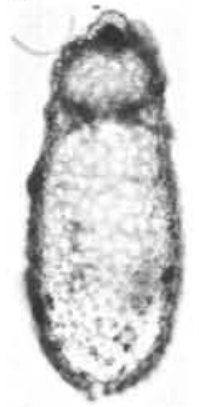

4

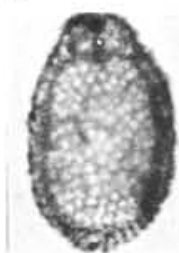

$8 a$

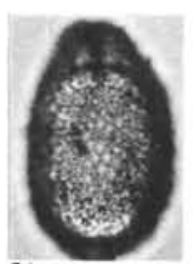

$8 b$

$5 a$

$9 a$

$10 \mathrm{a}$

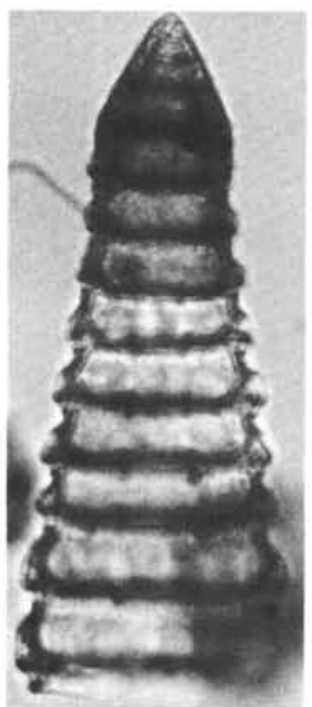

$1 \mathrm{~b}$
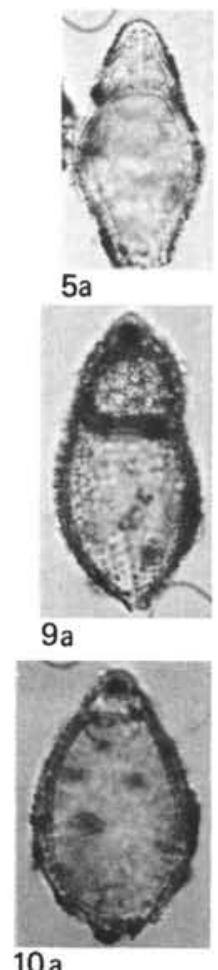

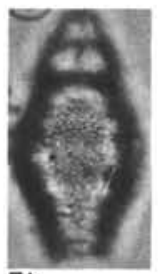

$5 b$

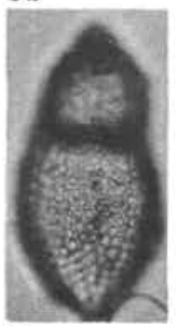

$9 b$

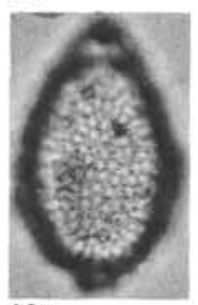

$10 \mathrm{~b}$

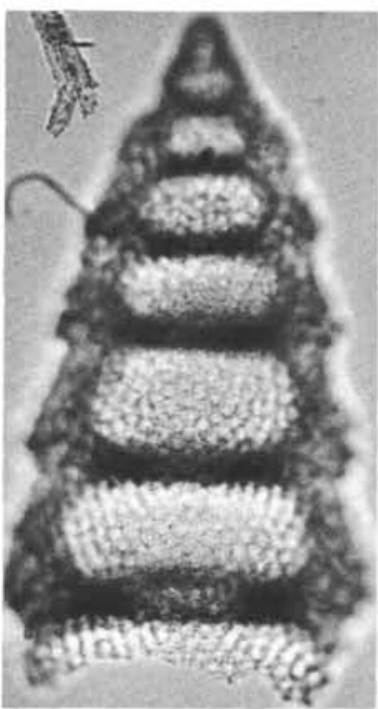

$2 a$

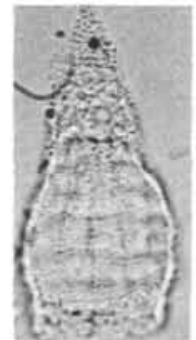

$6 a$

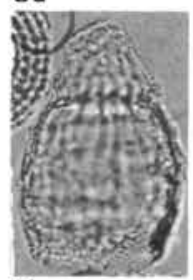

$11 \mathrm{a}$

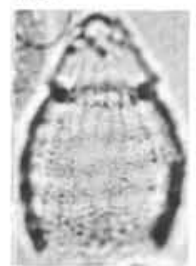

$11 \mathrm{~b}$

12

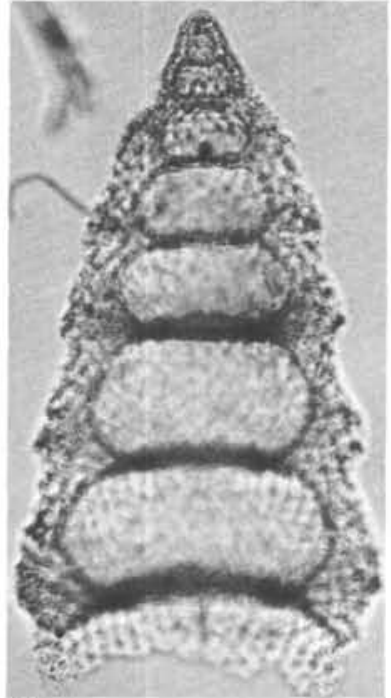

$2 b$
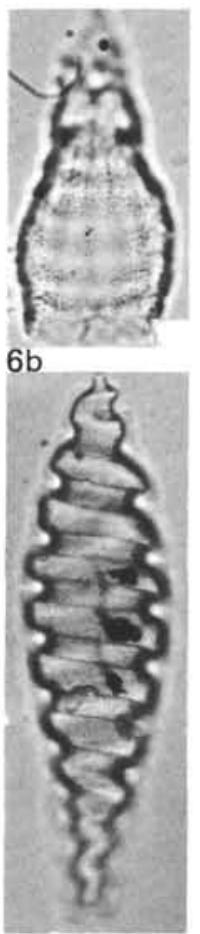

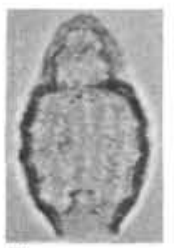

7

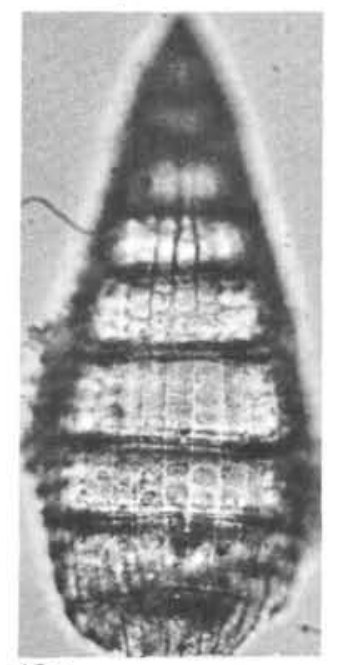

$13 a$

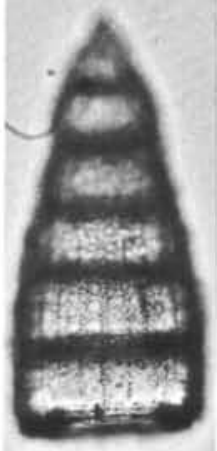

3a

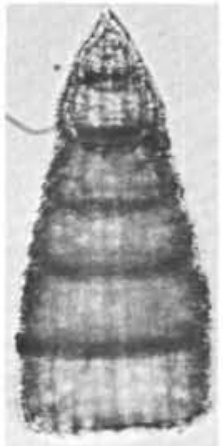

$3 b$

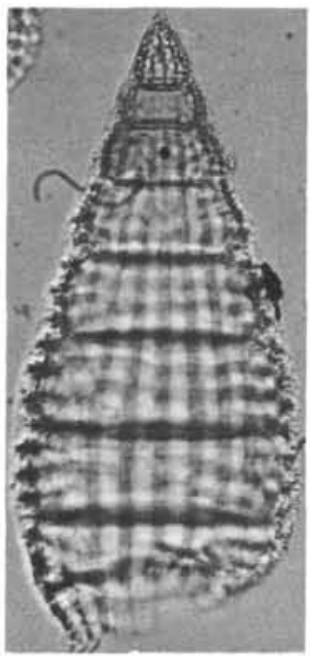

$13 b$

Plate 24. Photomicrographs.

Figures 1a, b. Pseudodictyomitra pseudomacrocephala (Squinabol) Sample 465A-29-1, 43-44 cm; slide 1; E.F. S 26/4.

Figures 2a, b. Spongocapsula? zamoraensis Pessagno. Sample 46634-2, 80-81 cm; slide 4; E.F. U 34/3.

Figures 3a, b. Mita sp. cf. M. magnifica Pessagno. Sample 465A-29-1, 43-44 cm; slide 4; E.F. B 34/3.

Figure 4. Theocapsomma ancus Foreman. Sample 465A-29-1, 43-44 $\mathrm{cm}$; slide 12 ; E.F. C $35 / 3$.

Figures $5 \mathrm{a}, \mathrm{b}$. Theocampe vanderhoofi Campbell and Clark. Sample 465A-29-1, 43-44 cm; slide 3; E.F. G 51/3.

Figures 6a, b. Artostrobium tina Foreman. Sample 466-34-2, 16-17 $\mathrm{cm}$; slide 6; E.F. X 34/2.

Figure 7. Theocampe salillum Foreman. Sample 466-34-2, 16-17 cm; slide 6; E.F. D 33/4.

Figures 8a, b. [?] Excentropylomma cenomana Dumitrică. Sample 465A-29-1, 43-44 cm; slide 7; E.F. E 36/4.

Figures $9 \mathrm{a}$, b. Theocapsomma ancus Foreman. Sample 465A-29-1, 43-44 cm; slide 6; E.F. S 43/1.

Figures 10a, b. Theocorys antiqua Squinabol. Sample 465A-29-1, 43-44 cm; slide 7; E.F. G 41/1.

Figures $11 \mathrm{a}, \mathrm{b}$. Theocampe sp. cf. $T$. vanderhoofi Campbell and Clark. Sample 466-34-2, 16-17 cm; slide 2; E.F. J 33/3.

Figure 12. Eucrytis sp. cf. E. tenuis. Note the spiral inner mold. Sample 463-72-5, 20-21 cm; slide 4; E.F. Q 38/4.

Figures 13a, b. Mita magnifica Pessagno. Sample 465A-29-1, 43-44 $\mathrm{cm}$; slide 17; E.F. O 32/4. 


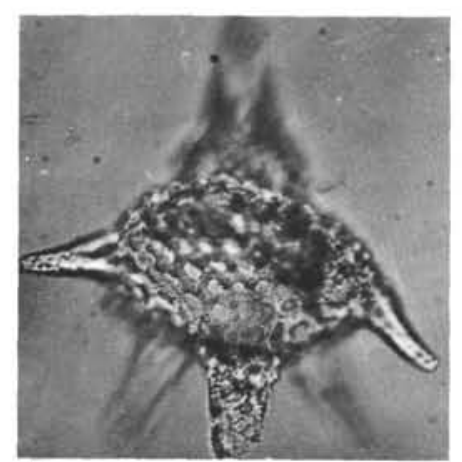

1a

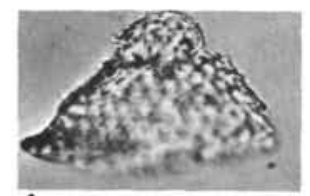

$4 a$

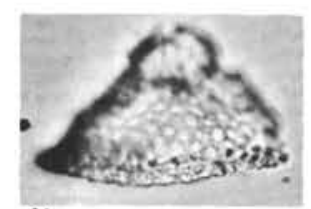

$4 b$

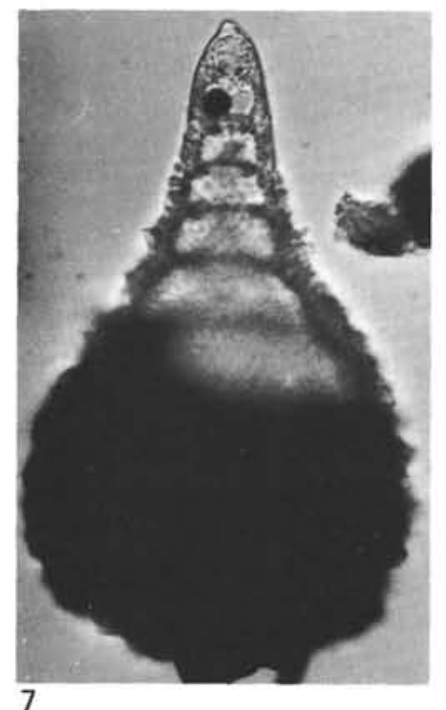

7

Plate 25. Photomicrographs.

Figures 1a, b. Podobursa triacantha (Fischli). Sample 463-89-1, 94-95 cm; slide 11; E.F. E 27/2.

Figures 2a, b. Podobursa tricola Foreman. Sample 463-89-1, 105-106 $\mathrm{cm}$; slide 8; E.F. C $43 / 2$.

Figures 3a, b. Eucyrtis elido, n. sp. Holotype. Sample 463-90,CC; slide 5; E.F. L 40/3.

Figures 4a, b. Neosciadocapsa sp. Sample 463-90,CC; slide 11; E.F. R 41/3.
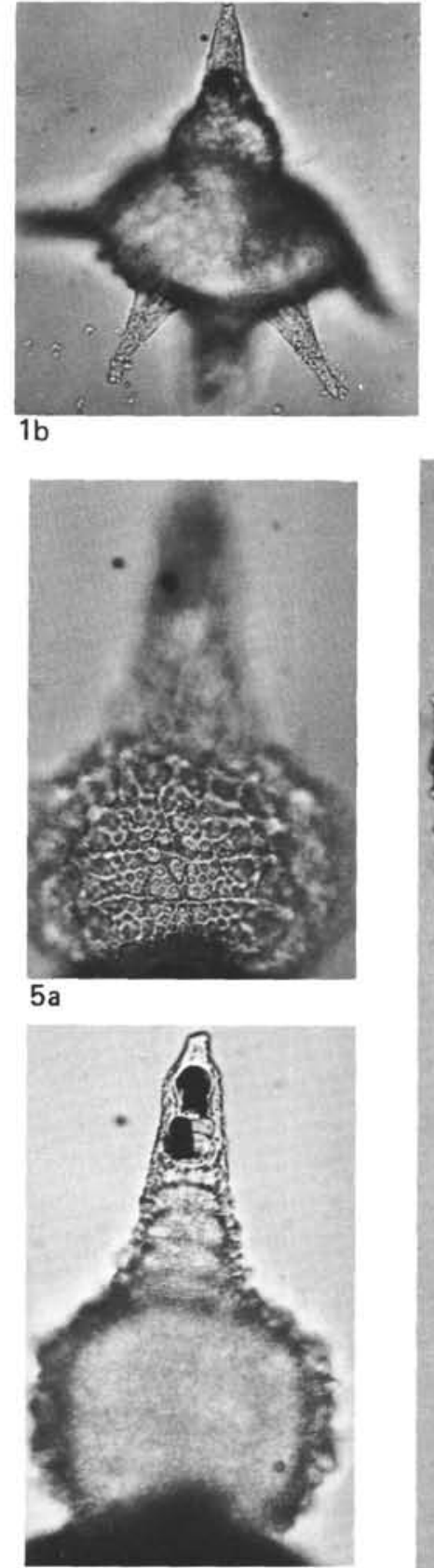

$5 \mathrm{~b}$

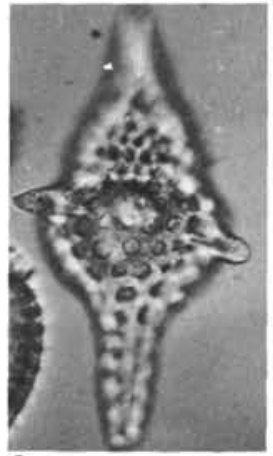

$2 \mathrm{a}$

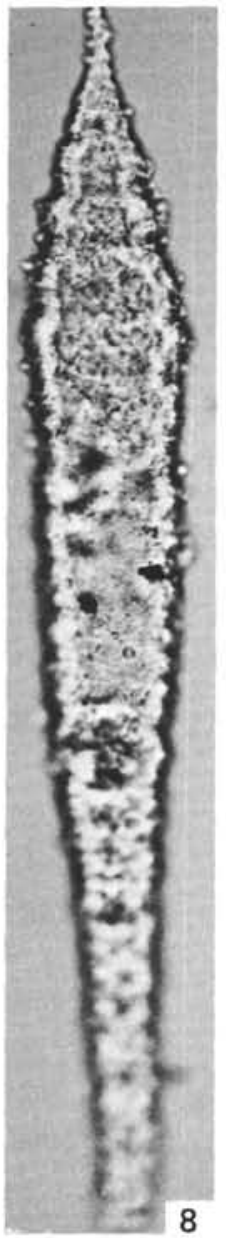

6

9 a

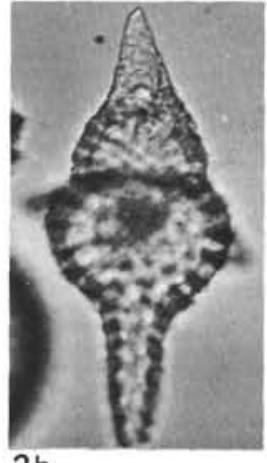

$2 b$
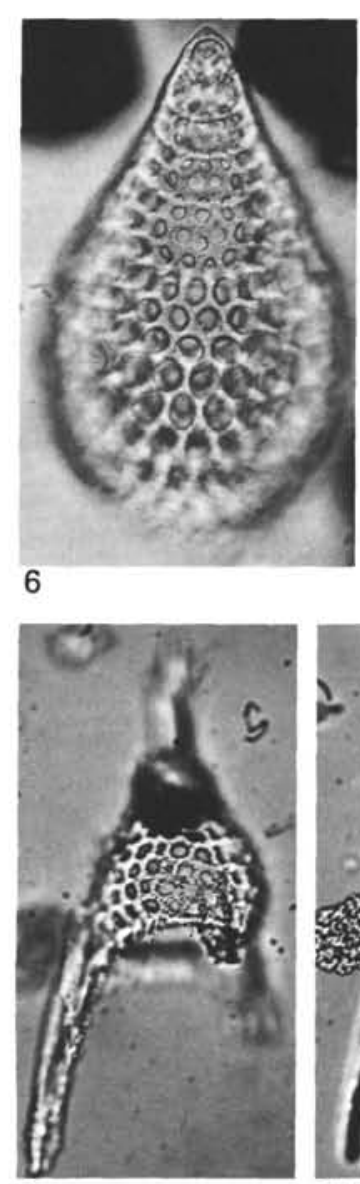

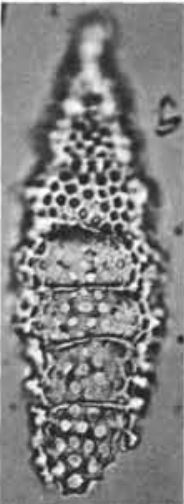

3a

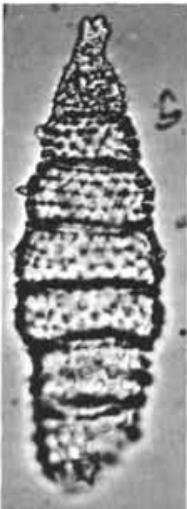

$3 b$

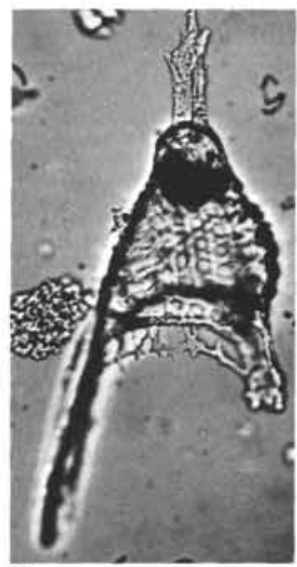

$9 b$

Figures 5a, b. Lithocampe chenodes Renz. Sample 463-89-1, 105-106 $\mathrm{cm}$; slide 9; E.F. D 14/2.

Figure 6. Stichocapsa cribata Hinde. Sample 463-89-1, 15-16 cm; slide 7; E.F. A $5 / 4$

Figure 7. Lithocampe chenodes Renz. Sample 463-89-1, 94-95 cm; slide 8; E.F. D 39/1.

Figure 8. Eucyrtis tenuis (Rüst). Sample 463-75-1, 21-22 cm; slide 26; E.F. H 29/4.

Figures 9a, b. Ultranapora spinifera Pessagno. Sample 463-90,CC; slide 10 ; E.F. X 31/3. 

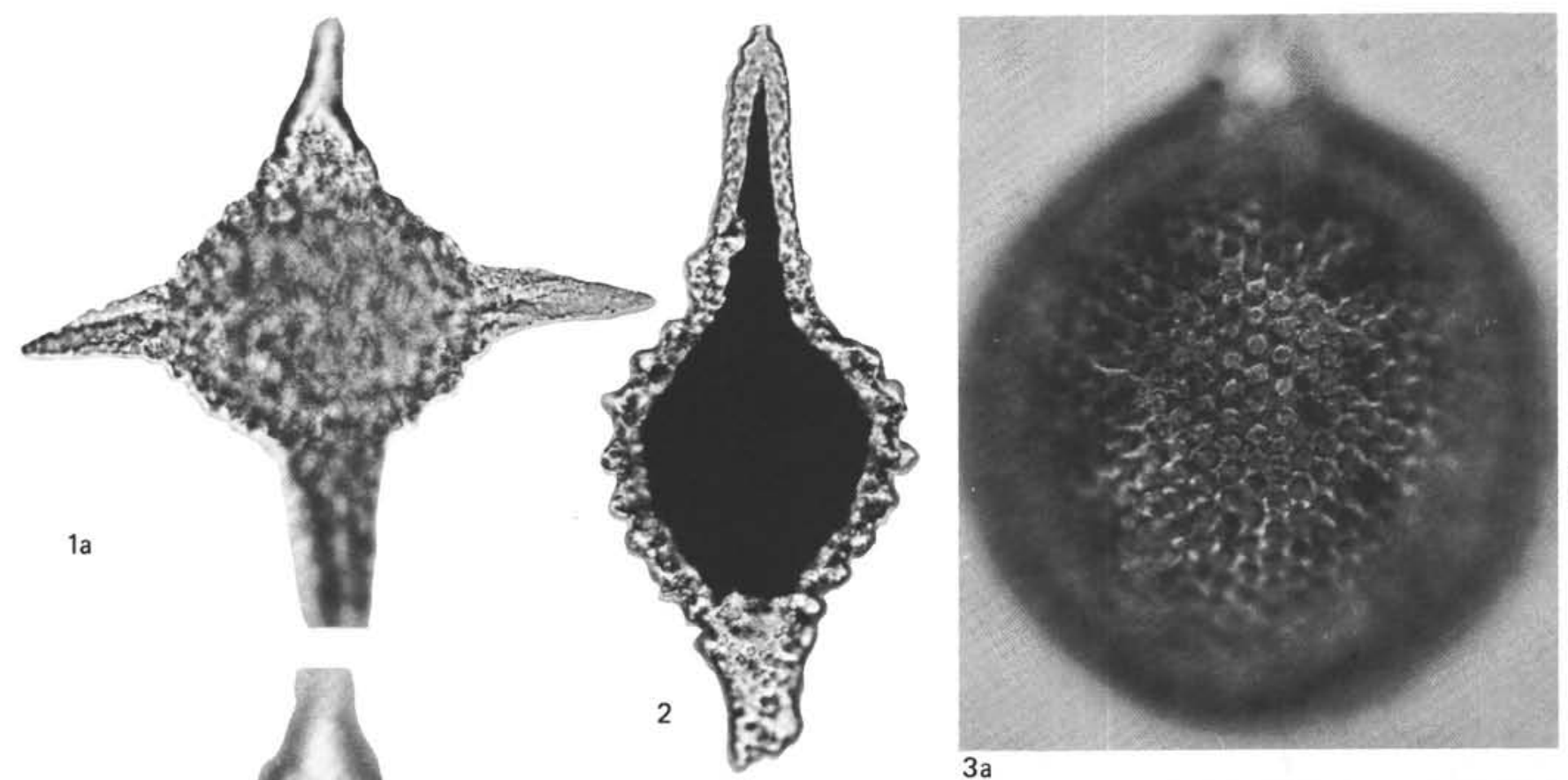

3a
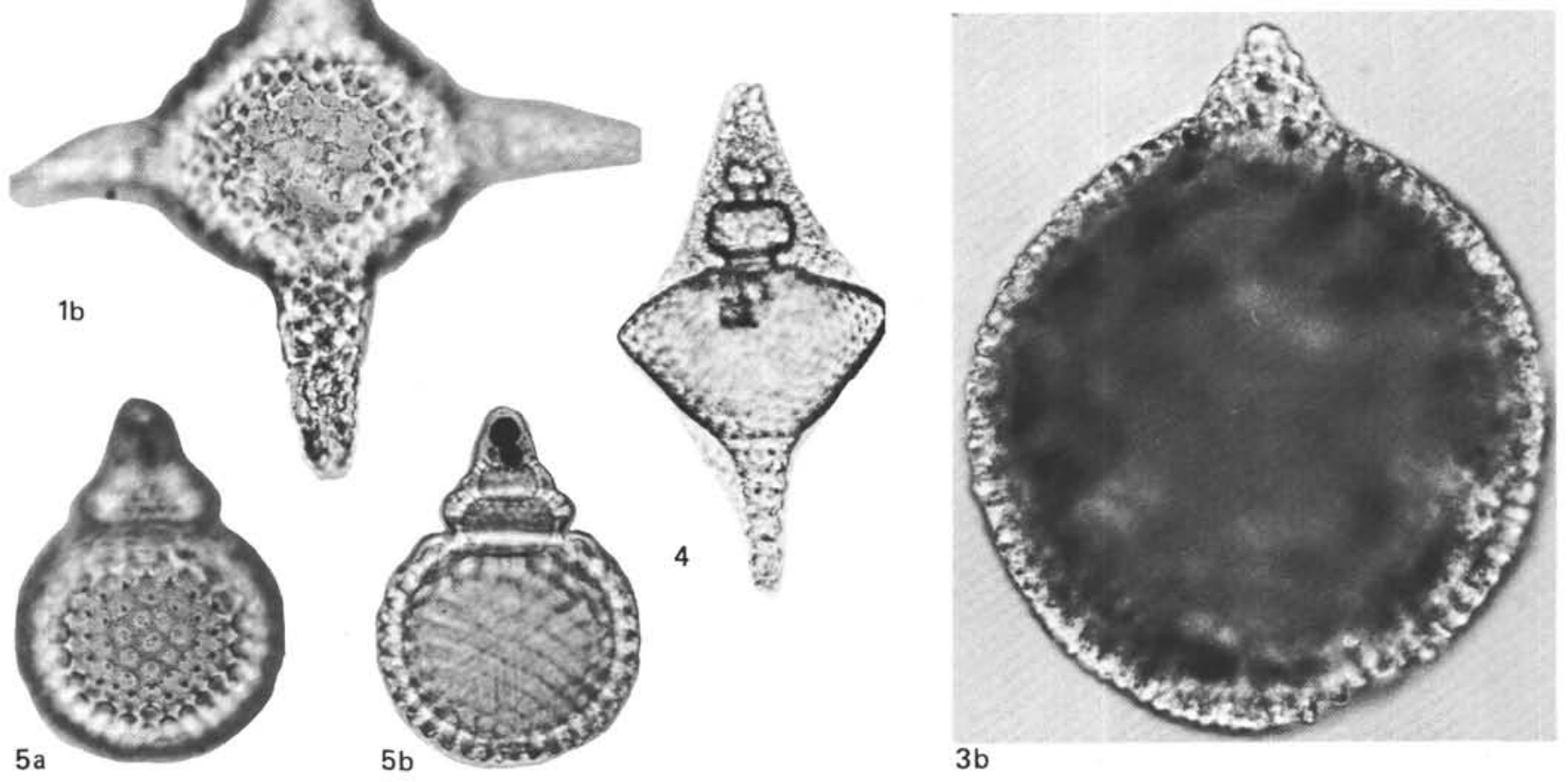

Plate 26. Photomicrographs.

Figures 1a, b. Dibolachras tytthopora Foreman. Sample 463-75-1, 21$22 \mathrm{~cm}$; slide 21; E.F. J 57/4.

Figure 2. Eucyrtis sp. cf. E. bulbosa Renz. Sample 463-75-1, 21-22 $\mathrm{cm}$; slide 23; E.F. V 48/4.
Figures 3a, b. Sethocapsa? orca Foreman. Sample 463-84-1, 5-6 cm; slide 8; E.F. Q 47/3.

Figure 4. Dibolachras tytthopora Foreman. Sample 463-73-1, 18-19 $\mathrm{cm}$; slide 5; E.F. G $47 / 3$.

Figures 5a, b. Sethocapsa uterculus (Parona). Sample 463-86-1, 31-32 $\mathrm{cm}$; slide 5; E.F. X 43/1. 


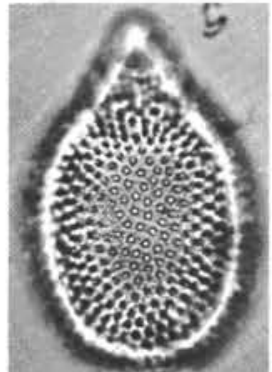

$1 a$

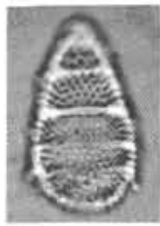

4 a

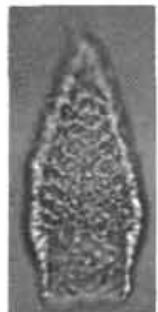

8 a

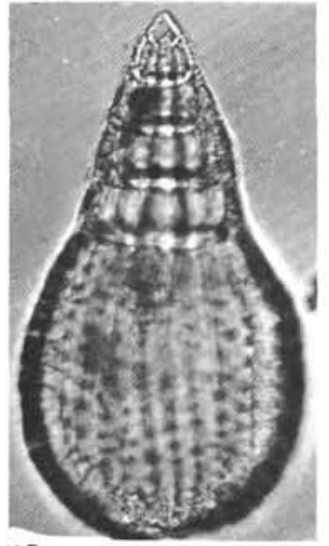

$12 \mathrm{a}$

ib

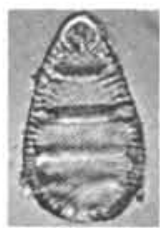

$4 b$

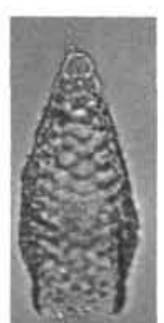

$8 b$
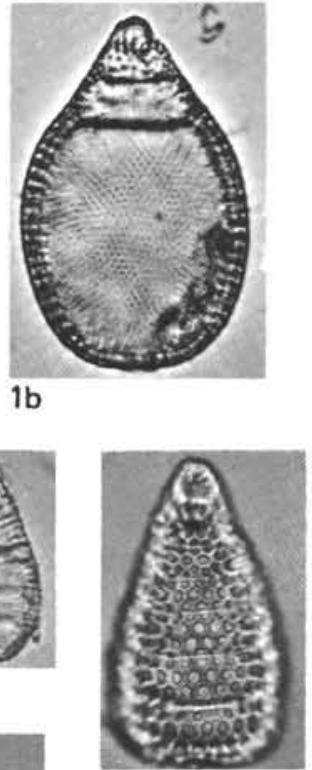

$5 a$

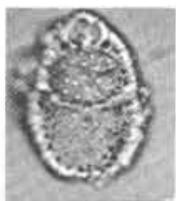

9

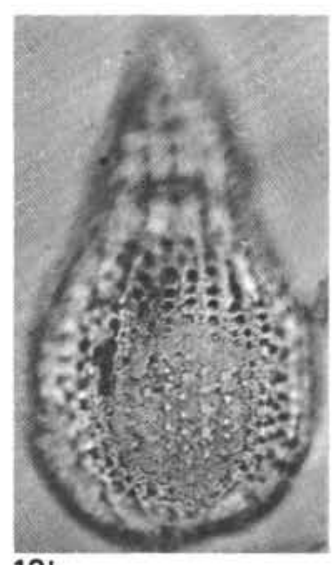

$12 b$

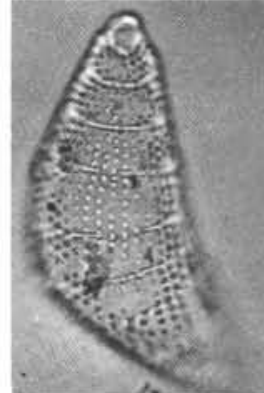

$2 a$

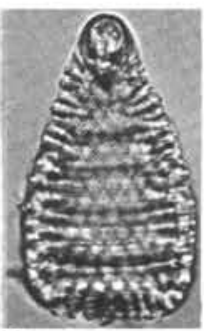

$5 b$

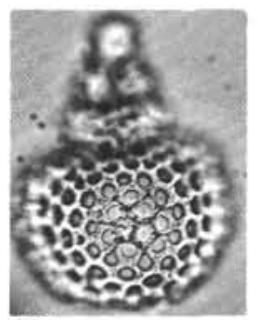

$10 \mathrm{a}$

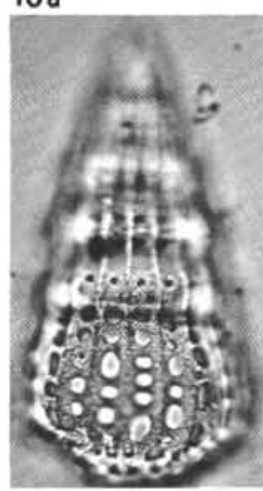

$13 a$

6a

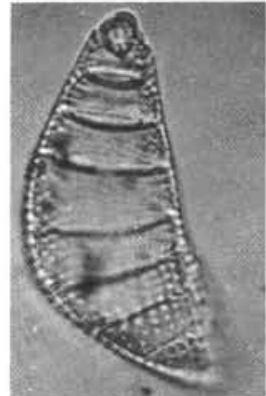

$2 \mathrm{~b}$
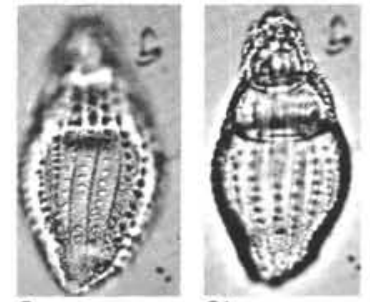

$6 b$

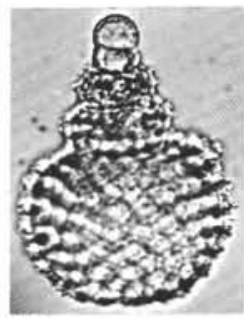

$10 \mathrm{~b}$

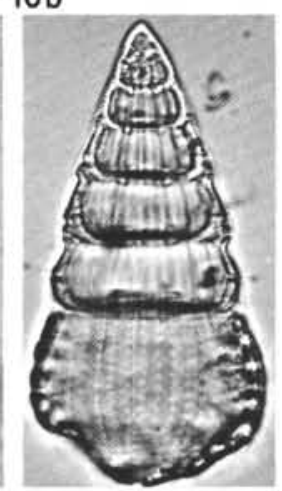

$13 b$

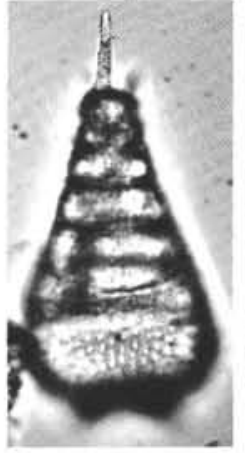

3a

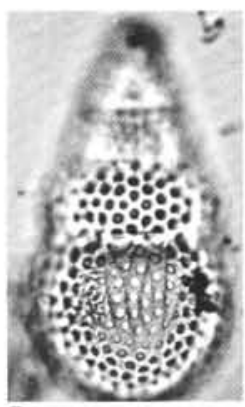

$7 a$

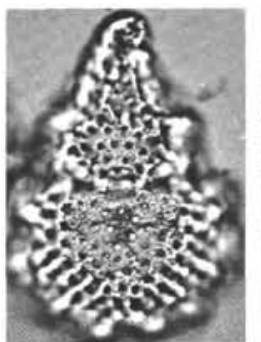

$11 \mathrm{a}$

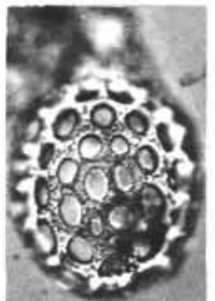

$14 a$

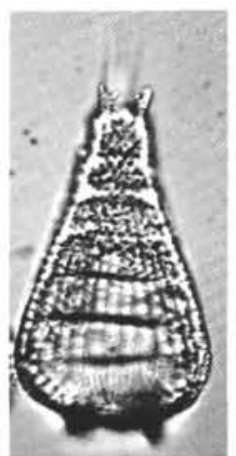

$3 b$
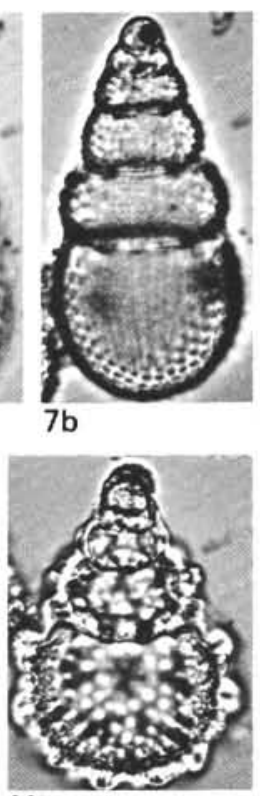

11b

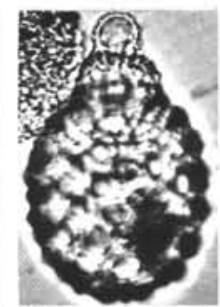

$14 b$
Plate 27. Photomicrographs.

Figures 1a, b. Theocorys renzae, n. sp. Holotype. Sample 463-90,CC; slide 9; E.F. W 44/4.

Figures 2a, b. Eucyrtis columbaria Renz. Lateral view. Sample 46389-1, 150-106 cm; slide 11; E.F. G 59/1.

Figures 3a, b. Eucyrtis columbaria Renz. Back view, showing the long apical horn and the two short subsidiary horns. Sample 463-90, CC; slide 4; E.F. F 30/3.

Figures $4 \mathrm{a}$, b. Stichocapsa pseudopentacola Tan Sin Hok. Sample 463-88-1, 52-53 cm; slide 5; E.F. L 34/3.

Figures 5a, b. Eucyrtis molengraaffi (Tan Sin Hok). Sample 46389-1, 105-107 cm; slide 2; E.F. T 45/2.

Figures 6a, b. Eucyrtidium thiensis Tan Sin Hok. Sample 463-90,CC; slide 2; E.F. Q 46/3.
Figures 7a, b. Stichocapsa pseudodecora Tan Sin Hok. Sample 46390 ,CC; slide 14; E.F. E 37/3.

Figures 8a, b. Cyrtocalpis operosa Tan Sin Hok. Sample 463-89-1, $105-106 \mathrm{~cm}$; slide 2; E.F. O 48/2.

Figure 9. Theocapsa laevis Tan Sin Hok. Sample 463-87-1, 6-7 cm; slide 2; E.F. W 35/1.

Figures 10a, b. Siphocampium davidi, n. sp. Holotype. Sample 463-90,CC; slide 6; E.F. E 29/1.

Figures 11a, b. Siphocampium rutteni (Tan Sin Hok). Sample 463-90, CC; slide 8; E.F. S 35/2.

Figures 12a, b. Stichocapsa sp. cf. S. decora Rüst. Sample 463-89-1, 23-24 cm; slide 2; E.F. S 54/1.

Figures 13a, b. Stichocapsa decora Rüst. Sample 463-90,CC; slide 8; E.F. K $28 / 4$.

Figures 14a, b. Siphocampium macropora (Rüst). Sample 463-90,CC; slide 12 ; E.F. P. $39 / 3$. 